\title{
Utilisation and outcomes of treatment in Autism Spectrum Disorder
}

Citation for published version (APA):

Houghton, R. (2021). Utilisation and outcomes of treatment in Autism Spectrum Disorder. [Doctoral Thesis, Maastricht University]. Gildeprint Drukkerijen. https://doi.org/10.26481/dis.20210304rh

Document status and date:

Published: 01/01/2021

DOI:

10.26481/dis.20210304rh

Document Version:

Publisher's PDF, also known as Version of record

\section{Please check the document version of this publication:}

- A submitted manuscript is the version of the article upon submission and before peer-review. There can be important differences between the submitted version and the official published version of record.

People interested in the research are advised to contact the author for the final version of the publication, or visit the DOI to the publisher's website.

- The final author version and the galley proof are versions of the publication after peer review.

- The final published version features the final layout of the paper including the volume, issue and page numbers.

Link to publication

\footnotetext{
General rights rights.

- You may freely distribute the URL identifying the publication in the public portal. please follow below link for the End User Agreement:

www.umlib.nl/taverne-license

Take down policy

If you believe that this document breaches copyright please contact us at:

repository@maastrichtuniversity.nl

providing details and we will investigate your claim.
}

Copyright and moral rights for the publications made accessible in the public portal are retained by the authors and/or other copyright owners and it is a condition of accessing publications that users recognise and abide by the legal requirements associated with these

- Users may download and print one copy of any publication from the public portal for the purpose of private study or research.

- You may not further distribute the material or use it for any profit-making activity or commercial gain

If the publication is distributed under the terms of Article $25 \mathrm{fa}$ of the Dutch Copyright Act, indicated by the "Taverne" license above, 


\section{Utilisation and Outcomes of Treatment in Autism Spectrum Disorder}

Richard Houghton 
ISBN: 9789464191097

Cover image: Tinnakorn Jorruang | www.shutterstock.com Lay-out and design: Daniëlle Balk | www.persoonlijkproefschrift.nl Printing: Gildeprint Enschede | www.gildeprint.n|

(c) Richard Houghton, 2021

All rights are reserved. No part of this thesis may be reproduced, distributed, stored in a retrieval system, or transmitted in any form or by any means, without prior written permission of the author. 


\section{UTILISATION AND OUTCOMES OF TREATMENT IN AUTISM SPECTRUM DISORDER}

To obtain the degree of Doctor at Maastricht University on the authority of the Rector Magnificus, Prof. dr. R.M Letschert in accordance with the Board of Deans to be defended in public on Thursday 4th March 2021 at 16:00 hours

by

Richard Houghton 


\section{Promotors:}

Prof. dr. F. de Vries

Prof. dr. J.P.W van den Bergh

\section{Assessment committee:}

Prof. dr. T.A.M.J. van Amelsvoort (Chairperson)

Prof. dr. H.G.M. Leufkens (Utrecht University)

Prof. dr. A.C.G. Egberts (Utrecht University)

Prof. dr. G.J.P. van Breukelen

Dr. P.K.C. Janssen 
For Auntie Angie, Caithan and Cade,

this thesis is for you and other families touched by autism. 



\section{Table of contents}

$\begin{array}{lll}\text { Chapter } 1 & \text { Introduction } & 9\end{array}$

$\begin{array}{lll}\text { Chapter } 2 & \text { Treatment utilisation in autism spectrum disorder } & 27\end{array}$

Chapter 2.1 Psychiatric comorbidities and use of psychotropic medications in people 29 with autism spectrum disorder in the United States

Chapter 2.2 Psychiatric comorbidities and psychotropic medication use in autism: a matched cohort study with ADHD and general population comparator groups in the United Kingdom

Chapter 2.3 Treatment patterns in children with autism in the United States

Chapter 3 Safety evaluation of treatments in children with autism spectrum 103 disorder

Chapter 3.1 Psychostimulants/atomoxetine and serious cardiovascular events in 105 children with ADHD or autism spectrum disorder

Chapter 3.2 Risperidone versus aripiprazole fracture risk in children and adolescents with autism spectrum disorder

Chapter 4 Validation of caregiver-reported severity of autism spectrum disorder

Chapter 4.1 Psychometric validation of the Autism Impact Measure (AIM)

Chapter 5 General discussion

Valorisation addendum

Databases used

Formal acknowledgements

Personal acknowledgements

List of publications 



\section{Chapter 1}

Introduction 


\section{Autism spectrum disorder}

Autism spectrum disorder (ASD) is a lifelong neurodevelopmental disorder, characterised by core symptoms of communication deficits, difficulties with social interaction, and restricted and repetitive behaviours. Affected people have difficulties in the ability to understand verbal and non-verbal social or emotional cues, as well as have repetitive patterns of behaviours or interests ${ }^{1}$. First described by a "basic desire for aloneness and sameness" and distinct from schizophrenia by Leo Kanner in $1943^{2}$, ASD has gone from being little-known outside the field of psychiatry to diagnosed in as many as 1 in 54 children ${ }^{3}$ and 1 in 45 adults ${ }^{4}$ in the USA today. Nowadays, ASD is known to affect people of all geographies and ethnic, cultural and racial backgrounds ${ }^{3-6}$. It can be diagnosed in children as young as 18 months old, yet is typically diagnosed at age 3-4 years in developed countries ${ }^{3,7}$. Approximately three to four times as many boys are diagnosed than girls ${ }^{8}$.

The underlying causes of ASD are poorly understood. Rather than having one specific cause, ASD likely reflects a group of distinct disorders influenced strongly by genetics and possibly by environmental factors ${ }^{9-13}$. Today, diagnoses are made based on clinical symptoms; however, large family and sibling studies estimate that between 64-91\% of effects are heritable $9,11,13$. Indeed, the concordance rate for ASD diagnoses is around $98 \%$ in monozygotic (identical) twins and $53 \%$ in dizygotic twins ${ }^{13}$. The rates are lower in non-twin siblings, but still far higher than in the general population ${ }^{14}$. Despite this evidence, no specific genomic variant of large effect is currently known to be implicated in more than $1 \%$ of cases ${ }^{11,15}$. Such alterations are also not exclusively implicated in ASD opposed to other neurological disorders such as intellectual disability (ID), bipolar, or epilepsy, pointing towards overlapping genetic aetiology ${ }^{11,16-18}$. Children with separate rare genetic syndromes such as Rett's syndrome or Fragile $X$ are at much higher risk of also being diagnosed with ASD or at least displaying some of the symptoms ${ }^{11}$. Increased maternal and paternal age have also been associated with increased risk ${ }^{19-22}$. Estimates for effects of environmental influences are much lower and tend to point the risk toward non-shared environments over shared environments (i.e. effects experienced by some but not all children in the same family) 9,10,13,23. Examples include maternal exposure to sodium valproate ${ }^{24}$, depression ${ }^{25,26}$ or infection ${ }^{27}$ during pregnancy, increased maternal body mass index ${ }^{28}$, season of birth ${ }^{29}$, long or short intervals between pregnancies ${ }^{30}$, caesarean delivery ${ }^{31}$ and low birth weight ${ }^{32,33}$. Evidence for causal relationships between autism and these environmental factors are generally amiss.

The variation in underlying ASD aetiology is likely what gives rise to the variation in its presentation. The manifestation and severity of symptoms vary with age ${ }^{11}$ and different developmental trajectories have been observed in the natural history of disease ${ }^{34,35}$. Some people with ASD will have severe language impairment throughout life, while others will develop abilities to read and speak fluently. Some autistic adults are able to live independently in the community whereas others require family or institutional help 
to perform daily activities ${ }^{15,36}$. Recent estimates are that around one-third of autistic children have comorbid ID, defined as having an intelligence quotient (IQ) of 70 points or less ${ }^{3}$. The proportion of patients diagnosed with ID has been decreasing in recent years as the prevalence of ASD has risen ${ }^{37}$. Other associated symptoms of autism - which also occur in some but not all cases - include obsessions, self-injury, irritability, aggression, and under-reactivity to sensory stimuli ${ }^{1,3,15}$. Psychiatric and neurological conditions such as attention deficit/hyperactivity disorder (ADHD), anxiety, depression, epilepsy and bipolar disorder are also more commonly diagnosed in children and adults with autism than in the general population ${ }^{38-42}$.

In 2013, to represent the wide range of known severities and symptoms, the American Psychiatric Association (APA) added the term "autism spectrum disorders" to the fifth edition of their Diagnostic and Statistical Manual of Mental Disorders (DSM-V) ${ }^{1}$. This definition replaced the previously established subtypes of autism including autistic disorder, Asperger syndrome, childhood disintegrative disorder (CDD) and pervasive developmental disorder not otherwise specified (PDD-NOS) and was intended to make diagnoses more straightforward ${ }^{15}$. While the underlying causes of autism may play some role in the increasing prevalence, other cited factors are greater awareness, less societal stigma and an expansion of the diagnostic criteria (not just in DSM-V but previously too) ${ }^{43,44}$. A number of diagnostic instruments are available to aid a professional diagnosis of ASD, including the Autism Diagnostic Observation Schedule (ADOS) ${ }^{45}$, the Autism Diagnostic Interview-Revised (ADI-R) ${ }^{46}$ and the Childhood Autism Rating Scale (CARS) ${ }^{47}$.

\section{Treatment landscape in ASD}

According to the American Academy of Pediatrics (AAP) the purposes of treatment in children with ASD should be to minimise core and associated ASD impairments, to maximise daily living skills for independence, and to relieve the impact of problem behaviours ${ }^{11}$. Goals are similar across the whole age range in ASD, including adults, but should be tailored dependent on verbal fluency, comorbidities and individual preferences and challenges ${ }^{48}$.

\section{Drug treatments}

There are currently no pharmacological treatment options for the core symptoms of ASD. In the USA, the only medications approved for associated symptoms - of irritability and aggression - are the atypical antipsychotics risperidone (since 2006 in children aged 5-16 years) and aripiprazole (since 2009 in children aged 6-17 years). Evidence leading to the approvals granted by the Food and Drug Administration (FDA) primarily came from randomised placebo-controlled trials, each with a double blind phase lasting only 8-weeks ${ }^{49-52}$ and a primary endpoint of the Aberrant Behaviour Checklist Irritabilitysubscale $(A B C-1)^{53}$. In Europe, based on the same data, the European Medicines Agency 
(EMA) granted risperidone market authorisation for "persistent aggression in conduct disorder in children" on the basis that it demonstrated efficacy in an associated, rather than a broad, set of ASD symptoms ${ }^{54}$. Although the EMA acknowledges aripiprazole demonstrated statistical superiority over placebo in randomised controlled trials (RCTS), the drug is not approved in ASD, as according to their review, the potential benefits of treatment did not outweigh risks ${ }^{55}$. The EMA summary of product characteristics for aripiprazole documents that the clinical relevance of the ABC-I has not been established and that there were concerns over weight gain and reductions in serum prolactin levels under treatment ${ }^{55}$.

Risperidone and aripiprazole have been recommended in clinical guidelines for treatment of aggression or self-injurious behaviours, in order to facilitate daily living, or to allow better adherence to non-drug therapies ${ }^{11,15,56}$. For example, the American Academy of Child and Adolescent Psychiatry (AACAP) recommend these treatments may be used but only with irritability symptoms ${ }^{56}$. The AAP recommend antipsychotics only when other behavioural factors and interventions have first been assessed, modified, and these modifications have not addressed concerns ${ }^{11}$. Similarly, despite there being no marketing authorisation in Europe, the UK National Institute for Health and Care Excellence (NICE) also recommends antipsychotic medications for children with behavioural challenges but only when non-drug treatments have not helped: but the antipsychotics should be started by a paediatrician or psychiatrist ${ }^{57}$.

Generally, it is acknowledged that other pharmacological treatments may be required to manage common comorbidities in ASD, but it is emphasised these treatments are not for ASD itself ${ }^{15,58}$. Comorbid neurological disorders should be managed in a way similar to if the patient did not have ASD ${ }^{15,57}$. These include for example, melatonin for sleep disturbances, stimulants for ADHD or selective serotonin reuptake inhibitors (SSRIs) for anxiety disorder or depression ${ }^{58}$. In the USA, psychotropic medications are extensively used to treat comorbid psychiatric comorbidities or other symptoms of ASD 59 . A recent systematic review reported median psychotropic drug use estimates of $42 \%$ in children and $62 \%$ in adults in ASD ${ }^{60}$. The systematic review also pointed to some clear gaps in the literature however. Firstly, the vast majority of studies only focused on North America and used data from over a decade ago. Furthermore, there are limited studies in the adult population and studies generally do not have non-ASD comparator groups to contextualise results.

\section{Safety concerns}

$\mathrm{ADHD}$ is the most common comorbidity in autism ${ }^{40}$. As such, drugs used for the treatment of ADHD, such as stimulants (e.g. amphetamine, methylphenidate, lisdexamfetamine) and atomoxetine are used frequently in ASD (around 14-19\% in children) ${ }^{60}$. Studied over many years, these drugs have demonstrated efficacy in the short-term management of ADHD symptoms ${ }^{61}$ but have consistently shown medication-induced increases in blood pressure 
and heart rate ${ }^{62}$. These findings, coupled with case reports ${ }^{63}$, have raised concerns over the potential increased risk of more serious cardiovascular (SCV) events such as stroke, myocardial infarction (MI), and cardiac arrhythmias ${ }^{64}$. A handful of observational studies in large samples have been conducted, but on data from over a decade ago, with limited statistical power due to rarity of outcomes, and have provided inconsistent results 65-70. Furthermore, children with ASD may be at increased risk of SCV events due to frequent use of concurrent medications, but until now, this subgroup has not been studied.

Antipsychotics are also commonly used by children and adolescents with ASD (around 17\%) ${ }^{60}$ and are known to be associated with important adverse effects such as weight gain, somnolence/sedation, and extrapyramidal disorders ${ }^{49-52}$. They are also commonly used among elderly dementia patients, and have been associated with increased bone fracture risk in this population ${ }^{71,72}$. Fractures are a leading cause of emergency room admission in children ${ }^{73}$ and the impact can be considerable, leading to reduction in daily functioning and exercise, lost days of schooling and increased chances of recurrent fractures in adulthood ${ }^{74,75}$. The relationship between antipsychotics and bone fractures may be due to an increased risk of accidents or falling ${ }^{76}$, or due to a negative impact on bone mineral density (BMD) ${ }^{77,78}$. The relationship between antipsychotics and fracture risk has not been well studied in children. However, as risperidone and aripiprazole are commonly used in ASD and have slightly different pharmacological profiles ${ }^{79}$, this setting can offer a unique opportunity to understand possible mechanisms and inform relevant clinical decisions about which treatment to prescribe.

\section{Non-drug treatments}

The mainstay of currently recommended treatments for ASD are non-pharmacological in nature, and include behavioural and social-communication based therapies 11,56,58. Emphasis is placed on early, intensive and individualised interventions, including speech, communication and adaptive skills training as needed, and parental involvement is encouraged ${ }^{11,56}$. Social and daily living skills training can be offered for both children and adults, either in groups or individually ${ }^{58}$.

In a study across 18 European countries, 91\% of children with ASD received at least one type of non-drug intervention by age 7 years, although there was wide variation in the types of treatments reported, perhaps due to variation in services offered by schools and respective national health systems ${ }^{80}$. Historical uptake of non-drug treatments is also high in the USA, with up to $77 \%$ current use ${ }^{81}$. However, there are concerns that children in more rural settings have access to fewer services ${ }^{82}$. Additionally, healthcare expenditures have previously been higher for ASD children with public versus private health insurance, suggesting public insurance is an advantage for accessing services ${ }^{83}$. Most states have introduced mandates requiring private insurance plans cover autism services ${ }^{44,84}$, but there has been little systematic research into current non-drug treatment patterns in the USA, or into understanding other barriers to receiving care. 


\section{Treatment utilisation}

Having reliable treatment utilisation estimates is important for a variety of reasons. They can be used to quantify disease burden, inform healthcare resource and training plans, measure the scale of certain risks (e.g. adverse events) and to aid planning for further research (such as defining eligibility criteria for new clinical trials). Other uses include identifying deviations from treatment guidelines or spotting differences in treatment approaches between countries, regions or healthcare plans. This can lead to additional understanding of underlying causes of deviations from guidelines, or if one healthcare system can learn from the other ${ }^{85}$.

\section{Assessing treatment effectiveness}

Finding new, safe and effective pharmacological interventions in ASD is an area of intense research. A recent search for "active" and "recruiting" phase 2 or 3 clinical trials in autism or ASD via the ClinicalTrials.gov website ${ }^{86}$ yielded 45 studies: 13 of which are industry sponsored. Novel drug mechanisms under investigation mainly involve neurobiological targets such as neurotransmitters: GABA and glutamate; or neuropeptides: oxytocin and vasopressin ${ }^{87,88}$. Other trials are on repurposed drugs from other disease areas, such as propranolol (a beta blocker) ${ }^{89}$ and bumetanide (a loop diuretic) ${ }^{90}$.

Unfortunately, there is little consensus on the most appropriate tools for measuring efficacy of treatments of ASD in clinical trials ${ }^{87}$. Some measures were developed and tested according to older and more narrow definitions of autism (e.g. Behavioral Summarized Evaluation Scale (BSE) ${ }^{91}$ and Real Life Rating Scale (RLRS) ${ }^{92}$ ) or focus on specific symptoms only (e.g. Social Responsiveness Scale (SRS) ${ }^{93}$ and Repetitive Behaviours Scale-Revised (RBS-R) ${ }^{94}$ ). Furthermore, interview-administered measures such as the Vineland Adaptive Behavior Scales (VABS) ${ }^{95,96}$, often require trained personal to administer, making them expensive and time-consuming. Reliable measures for real world monitoring of ASD symptoms beyond the timeframe and setting of clinical trials are also lacking.

In summary, the treatment landscape in ASD is complex. Many research questions remain unanswered with regards to levels of treatment utilisation, safety profiles of commonly used medications and a lack of appropriate tools for assessing treatment effectiveness. 


\section{Real world data}

Real world data (RWD) can be broadly classified as any data collected outside the realm of strictly controlled settings like RCTs. Often cited advantages are that RWD can be collected more efficiently and cheaply, leading to opportunities to access data from large numbers of people ${ }^{97}$. Additional advantages of RWD make this data more intrinsically valuable for certain research questions too.

Firstly, clinical trials often have strict inclusion criteria that restricts the eligibility of participants. For example, eligibility can be restricted to a certain age range, or to patients without other health conditions than the one being studied. Therefore, RCT study entry criteria may be unrepresentative of all patients likely to receive treatment in a real life setting. Given ASD is a lifelong disorder and many patients have comorbidities, this can be quite an issue for ASD studies. Furthermore, the way patients access and use treatments in RCTs is different to real life practice by design. Hence, the effects of socio-economic factors like family income, geography or insurance type on the ability to even access treatment is only possible to study by using RWD. Adherence to medications in real life practice is also known to be lower than in clinical trials, and especially so in psychiatric diseases or in patients with cognitive disabilities ${ }^{98,99}$.

Secondly, it is not always possible to ascertain good estimates of potential adverse drug effects in small cohorts or in short periods of follow-up afforded by RCTs. The statistical logic of primary study outcomes is often not applied to adverse events. Consider rare adverse events mentioned above like serious cardiovascular events, which only occur in approximately 1 per 30,000 patient years for children with neurodevelopmental disorders 65,70 or effects that are only biologically plausible after longer periods of drug exposure, such as reduced BMD leading to fractures. Studying these potential effects of treatment is simply not possible over the short timeframe of most clinical trials.

Finally, it is of interest to study the effectiveness of different interventions under real life conditions and over longer periods of use. By examining RWD from a large body of patients being exposed to a treatment for longer periods of time, both adverse events and expected (or even unexpected) benefits can be appreciated. Patient or caregiver-reported assessments can offer a quicker and cheaper option to abovementioned tools often used in clinical trials, which may need trained personal or a longer duration to administer. They may also be administered remotely, for example on a computer or mobile phone. Hence, provided they are validated, they can offer a more sustainable opportunity to evaluate treatment effectiveness over longer periods, and outside controlled clinical trial settings. Another advantage of using such tools in a real world setting is that they may also provide more rapid feedback to patients, caregivers and physicians, in order to effectively find optimal treatment strategies on the individual level ${ }^{88}$. 


\section{Secondary use data}

Some research questions can be answered using RWD that already exist. Such databases typically come into being via data entry on the individual level, but without much forethought of the research questions that data could address at an aggregate, population level. Examples of such databases are electronic medical records (EMRs) and medical insurance claims databases.

\section{Clinical Practice Research Datalink}

EMR data are routinely collected by doctors or other healthcare professionals to keep record of their patients' medical history. One of the largest and most well established EMRs worldwide is the Clinical Practice Research Datalink (CPRD) database from the UK. The data are captured in the primary care setting by general practitioners (GP), who are seen as the "gatekeepers" to access other non-emergency care provided by the National Health Service (NHS) ${ }^{100}$. As such, GPs play a key role in the management of services for people with ASD and the CPRD data contains patient demographics, consultations, diagnoses and prescriptions from primary care, as well as key referrals to, and diagnoses from secondary care ${ }^{15,100,101}$. The number of patients included in the database has changed over time, but includes 14 million currently active patients as of $2020{ }^{102}$. The data are representative of the UK population in terms of age, sex and ethnicity ${ }^{100}$. ASD diagnoses recorded in the CPRD are reliable and can be used with confidence ${ }^{103}$. The data have been used in over 2,500 peer-reviewed publications ${ }^{102}$, including to demonstrate the lack of association between autism and the measles-mumps-rubella (MMR) vaccination ${ }^{104}$.

\section{MarketScan insurance claims database}

Medical insurance claims provide an alternative source of real world data. The IBM MarketScan ${ }^{\circledR}$ databases ${ }^{105}$ cover de-identified patient-level health data from private and publically insured populations from the USA. Information recorded comprise billed episodes of care from all settings, including retail, mail order and specialty pharmacy prescriptions. The MarketScan "commercial" dataset is representative of employees and dependents of mid to large sized companies, with company-sponsored private medical insurance. This can include dependants with ASD. The MarketScan Medicaid dataset covers the Medicaid public insurance program from around 10-12 states. Medicaid is a state-run health insurance for people of lower income families or with certain qualifying disabilities, including ASD. Each MarketScan database covers several million enrolees per year, and the data have been used in over 2,000 peer-reviewed publications ${ }^{105}$.

\section{Primary data collection and the SPARK study}

Because EMR and claims data are not primarily collected for the purposes of medical research, they can lack important information. Such data includes detailed clinical characteristics, treatments received outside of the service network, societal or economic determinants of health, and concepts such as patient or caregiver opinions and preferences. In some circumstances, additional information may need to be gathered 
proactively and systematically via a primary data collection study. Registry studies and surveys are primary data collection methods which are typically low-interventional in nature. They are hence cheaper to conduct than clinical trials yet maintain the advantages of representing real life clinical practice.

Simons Foundation Powering Autism Research for Knowledge (SPARK) is a USA based online research initiative for individuals with ASD and their family members, who have consented to providing information and medical samples to further autism research ${ }^{106}$. Established in 2016 by the Simons Foundation Autism Research Initiative (SFARI), almost 60,000 individuals with ASD enrolled by the end of $2018{ }^{107}$ and recruitment continues today. Whole exome sequencing studies identifying additional ASD risk genes are starting to be published from this data ${ }^{108}$. Other examples of research based on the cohort include understanding medical profiles of ASD ${ }^{107}$, beliefs in vaccines as a cause of autism 109 and the study of coordination disorders in ASD ${ }^{110}$. SFARI facilitates the opportunity for external researchers to collect additional information from SPARK participants via electronic surveys. This is only provided if the data is later returned and made linkable to other data from the same cohort in order to aid additional research. In this sense, the database allows cross-sectional and longitudinal assessment of the cohort.

\section{Objectives and outline of this thesis}

This thesis comprises a collection of studies to address various abovementioned knowledge gaps regarding the utilisation and outcomes of treatment in ASD. The goals can be grouped into the following three categories: treatment utilisation patterns, treatment safety, and validation of a new caregiver-reported measure of ASD symptom severity. Details of the thesis outline and sub-goals of each chapter are outlined in Figure 1.1. 
Figure 1.1: Outline of this thesis

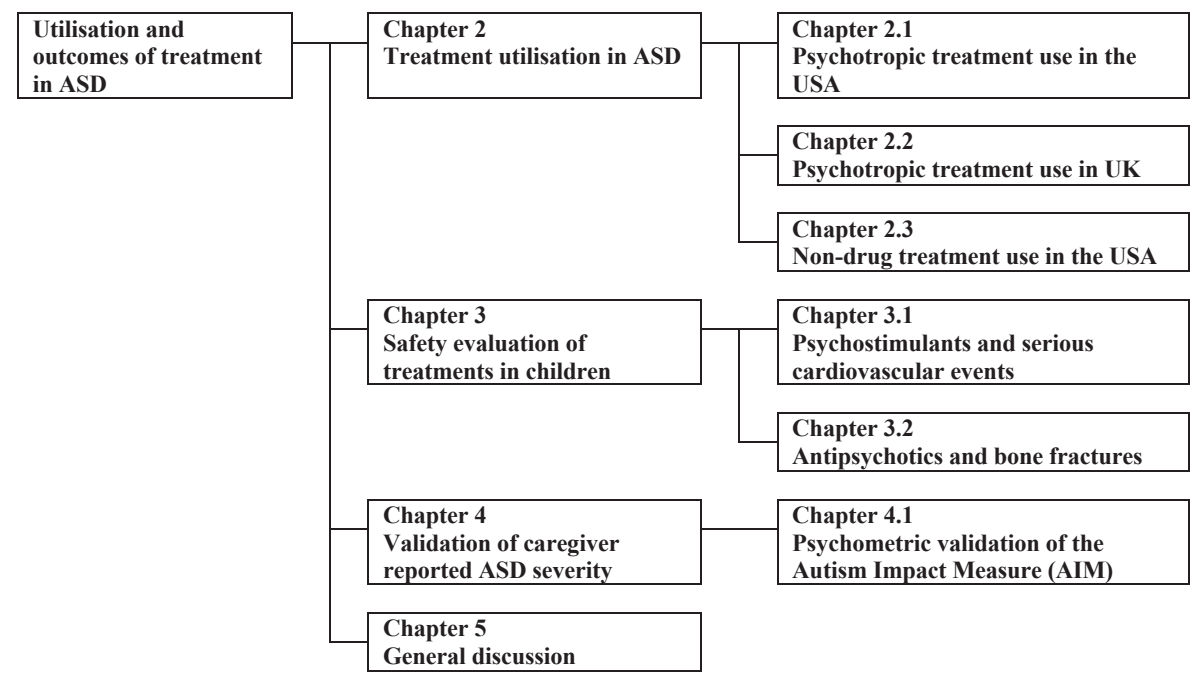

$\mathrm{ADHD}=$ attention deficit hyperactive disorder; $\mathrm{ASD}=$ autism spectrum disorder

Chapter 2 focuses on the production of up to date estimates of drug and non-drug treatment use in ASD. We extend the current literature by studying psychotropic drug use in the adult ASD population as well as children. Furthermore, we estimate treatment use in the USA and the UK, assess predictors of treatment use, and make comparisons to reference groups without ASD to contextualise our findings. For non-drug treatments, we evaluate the prevalence, intensity, setting and barriers to care for different treatment types in the USA, and test if geography or insurance type is associated with treatment use.

Chapter $\mathbf{3}$ explores safety concerns of commonly used drug treatments in children with ASD. Firstly, we quantify the risk of serious cardiovascular events in ASD and ADHD and assess if these events are associated with ADHD medication use. Secondly, we compare the risk of bone fractures head-to-head between risperidone and aripiprazole: two of the most commonly used antipsychotic treatments in ASD, but with differing pharmacological profiles.

Chapter 4 is dedicated to the psychometric validation of a new caregiver reported assessment for the severity of ASD symptoms in children.

Chapter $\mathbf{5}$ is a summary and critical reflection on the main body of this thesis. It includes a summary of the main findings, a critical evaluation of epidemiological methods used and considerations for future research and implications. 


\section{References}

1. American Psychiatric Association. Diagnostic and Statistical Manual of Mental Disorders (5th ed.). (Washington, DC; Author, 2013).

2. Kanner, L. Autistic disturbances of affective contact. Nerv. Child 2, 217-250 (1943).

3. Maenner, M. J.et al. Prevalence of Autism Spectrum Disorder Among Children Aged 8 Years - Autism and Developmental Disabilities Monitoring Network, 11 Sites, United States, 2016. MMWR Surveill. Summ. 69, 1-12 (2020).

4. Dietz, P. M., Rose, C. E., McArthur, D. \& Maenner, M. National and State Estimates of Adults with Autism Spectrum Disorder. J. Autism Dev. Disord. (2020) doi:10.1007/s10803-020-04494-4.

5. Elsabbagh, M.et al. Global Prevalence of Autism and Other Pervasive Developmental Disorders. Autism Res. 5, 160179 (2012).

6. Rice, C. E. \& Lee, L.-C. Expanding the global reach of research in autism. Autism 21, 515-517 (2017).

7. Salomone, E., Charman, T., McConachie, H. \& Warreyn, P. Child's verbal ability and gender are associated with age at diagnosis in a sample of young children with ASD in Europe. Child Care Health Dev. 42, 141-145 (2016).

8. Loomes, R., Hull, L. \& Mandy, W. P. L. What Is the Male-to-Female Ratio in Autism Spectrum Disorder? A Systematic Review and Meta-Analysis. J. Am. Acad. Child Adolesc. Psychiatry 56, 466-474 (2017).

9. Bai, D. et al. Association of Genetic and Environmental Factors With Autism in a 5-Country Cohort. JAMA Psychiatry (2019) doi:10.1001/jamapsychiatry.2019.1411.

10. Colvert, E. et al. Heritability of Autism Spectrum Disorder in a UK PopulationBased Twin Sample. JAMA Psychiatry 72, 415-423 (2015).
11. Hyman, S. L., Levy, S. E., Myers, S. M. \& Council on Children with Disabilities, S. on D. and B. P. Identification, Evaluation, and Management of Children With Autism Spectrum Disorder. Pediatrics 145, e20193447 (2020).

12. Rylaarsdam, L. \& Guemez-Gamboa, A. Genetic Causes and Modifiers of Autism Spectrum Disorder. Front. Cell. Neurosci. 13, 385 (2019).

13. Tick, B., Bolton, P., Happé, F., Rutter, M. \& Rijsdijk, F. Heritability of autism spectrum disorders: a meta-analysis of twin studies. J. Child Psychol. Psychiatry 57, 585-595 (2016).

14. Xie, F., Peltier, M. \& Getahun, D. Is the Risk of Autism in Younger Siblings of Affected Children Moderated by Sex, Race/Ethnicity, or Gestational Age? J. Dev. Behav. Pediatr. 37, 603-609 (2016).

15. Lord, C., Elsabbagh, M., Baird, G. \& Veenstra-Vanderweele, J. Autism spectrum disorder. The Lancet 392, 508-520 (2018).

16. Gonzalez-Mantilla, A. J., Moreno-DeLuca, A., Ledbetter, D. H. \& Martin, C. L. A Cross-Disorder Method to Identify Novel Candidate Genes for Developmental Brain Disorders. JAMA Psychiatry 73, 275-283 (2016).

17. Li, J.et al. Genes with de novo mutations are shared by four neuropsychiatric disorders discovered from NPdenovo database. Mol. Psychiatry 21, 290-297 (2016).

18. Myers, S. M. et al. Insufficient Evidence for 'Autism-Specific' Genes. Am. J. Hum. Genet. 106, 587-595 (2020).

19. Hultman, C. M., Sandin, S., Levine, S. Z., Lichtenstein, P. \& Reichenberg, A. Advancing paternal age and risk of autism: new evidence from a populationbased study and a meta-analysis of epidemiological studies. Mol. Psychiatry 16, 1203-1212 (2011). 
20. Sandin, S. et al. Advancing maternal age is associated with increasing risk for autism: a review and meta-analysis. J. Am. Acad. Child Adolesc. Psychiatry 51, 477-486.e1 (2012).

21. Sandin, S. et al. Autism risk associated with parental age and with increasing difference in age between the parents. Mol. Psychiatry 21, 693-700 (2016).

22. Wu, S. et al. Advanced parental age and autism risk in children: a systematic review and meta-analysis. Acta Psychiatr. Scand. 135, 29-41 (2017).

23. Sandin, S. et al. The Heritability of Autism Spectrum Disorder. JAMA 318, 11821184 (2017).

24. Christensen, J. et al. Prenatal Valproate Exposure and Risk of Autism Spectrum Disorders and Childhood Autism. JAMA 309, 1696-1703 (2013).

25. Hagberg, K. W., Robijn, A. L. \& Jick, S. Maternal depression and antidepressant use during pregnancy and the risk of autism spectrum disorder in offspring. Clin. Epidemiol. 10, 1599-1612 (2018).

26. Mezzacappa, A. et al. Risk for Autism Spectrum Disorders According to Period of Prenatal Antidepressant Exposure: A Systematic Review and Meta-analysis. JAMA Pediatr. 171, 555-563 (2017).

27. Al-Haddad, B. J. S. et al. Long-term Risk of Neuropsychiatric Disease After Exposure to Infection In Utero. JAMA Psychiatry 76, 594-602 (2019).

28. Li, Y.-M. et al. Association Between Maternal Obesity and Autism Spectrum Disorder in Offspring: A Meta-analysis. J. Autism Dev. Disord. 46, 95-102 (2016).

29. Lee, B. K. et al. Birth seasonality and risk of autism spectrum disorder. Eur. J. Epidemiol. 34, 785-792 (2019).

30. Schieve, L. A. et al. Autism spectrum disorder and birth spacing: Findings from the study to explore early development (SEED). Autism Res. 11, 81-94 (2018).

31. Zhang, T. et al. Association of Cesarean Delivery With Risk of Neurodevelopmental and Psychiatric Disorders in the Offspring: A Systematic Review and Meta-analysis. JAMA Netw. Open 2, e1910236-e1910236 (2019).
32. Brumbaugh, J. E., Weaver, A. L., Myers, S. M., Voigt, R. G. \& Katusic, S. K. Gestational Age, Perinatal Characteristics, and Autism Spectrum Disorder: A Birth Cohort Study. J. Pediatr. 220, 175-183.e8 (2020).

33. Lampi, K. M. et al. Risk of Autism Spectrum Disorders in Low Birth Weight and Small for Gestational Age Infants. J. Pediatr. 161, 830-836 (2012).

34. Fountain, C., Winter, A. S. \& Bearman, P. S. Six Developmental Trajectories Characterize Children With Autism. Pediatrics 129, e1112-e1120 (2012).

35. Szatmari, P. et al. Developmental Trajectories of Symptom Severity and Adaptive Functioning in an Inception Cohort of Preschool Children With Autism Spectrum Disorder. JAMA Psychiatry 72, 276-283 (2015).

36. Maenner, M. J. et al. Evaluation of an activities of daily living scale for adolescents and adults with developmental disabilities. Disabil. Health J. 6, 8-17 (2013).

37. Thurm, A., Farmer, C., Salzman, E., Lord, C. \& Bishop, S. State of the Field: Differentiating Intellectual Disability From Autism Spectrum Disorder. Front. Psychiatry 10, 526 (2019).

38. Buck, T. R. et al. Psychiatric comorbidity and medication use in adults with autism spectrum disorder. J. Autism Dev. Disord. 44, 3063-3071 (2014).

39. Joshi, G. et al. Psychiatric comorbidity and functioning in a clinically referred population of adults with autism spectrum disorders: a comparative study. J. Autism Dev. Disord. 43, 1314-1325 (2013).

40. Lai, M.-C. et al. Prevalence of cooccurring mental health diagnoses in the autism population: a systematic review and meta-analysis. Lancet Psychiatry 6, 819-829 (2019).

41. Lugo-Marín, J. et al. Prevalence of psychiatric disorders in adults with autism spectrum disorder: A systematic review and meta-analysis. Res. Autism Spectr. Disord. 59, 22-33 (2019). 
42. Simonoff, E. et al. Psychiatric disorders in children with autism spectrum disorders: prevalence, comorbidity, and associated factors in a population-derived sample. J. Am. Acad. Child Adolesc. Psychiatry 47, 921-929 (2008).

43. Hodges, H., Fealko, C. \& Soares, N. Autism spectrum disorder: definition, epidemiology, causes, and clinical evaluation. Transl. Pediatr. 9, S55-S65 (2020).

44. Mandell, D. S. et al. Effects of Autism Spectrum Disorder Insurance Mandates on the Treated Prevalence of Autism Spectrum Disorder. JAMA Pediatr. 170, 887-893 (2016).

45. Lord, C. et al. Autism diagnostic observation schedule, second edition. (Western Psychological Services, 2012).

46. Rutter, M., LeCouteur, A. \& Lord, C. (ADIR) Autism Diagnostic Interview-Revised. (Western Psychological Services, 2003).

47. Dawkins, T., Meyer, A. T. \& Van Bourgondien, M. E. The Relationship Between the Childhood Autism Rating Scale: Second Edition and Clinical Diagnosis Utilizing the DSM-IV-TR and the DSM-5. J. Autism Dev. Disord. 46, 3361-3368 (2016).

48. Lord, C. et al. Autism spectrum disorder. Nat. Rev. Dis. Primer 6, 1-23 (2020).

49. Marcus, R. N.et al. A Placebo-Controlled, Fixed-Dose Study of Aripiprazole in Children and Adolescents With Irritability Associated With Autistic Disorder. J. Am. Acad. Child Adolesc. Psychiatry 48, 1110-1119 (2009).

50. McCracken, J. T. et al. Risperidone in children with autism and serious behavioral problems. N. Engl. J. Med. 347, 314-321 (2002).

51. Owen, R. et al. Aripiprazole in the treatment of irritability in children and adolescents with autistic disorder. Pediatrics 124, 1533-1540 (2009).

52. Shea, S.et al. Risperidone in the treatment of disruptive behavioral symptoms in children with autistic and other pervasive developmental disorders. Pediatrics 114, e634-641 (2004).
53. Aman, M. \& Singh, N. Aberrant Behavior Checklist: Manual. (New York, NY; Slosson Educational Publications, 1986).

54. European Medicines Agency. Risperidal - Article 30 referral - ANNEX I, II, III, IV. https://www.ema.europa.eu/en/ documents/referral/risperdal-article30-referral-annex-i-ii-iii-iv_en-0.pdf; Accessed 12 Jul 2020.

55. European Medicines Agency. AbilifyProcedural steps taken and scientific information after the authorisation EMA/248614/2020. https://www.ema. europa.eu/en/documents/proceduralsteps-after/abilify-epar-proceduralsteps-taken-scientific-information-afterauthorisation_en.pdf; Accessed 20 Jul 2020.

56. Volkmar, F. et al. Practice parameter for the assessment and treatment of children and adolescents with autism spectrum disorder. J. Am. Acad. Child Adolesc. Psychiatry 53, 237-257 (2014).

57. NICE. Autism spectrum disorder in under 19s: support and management [CG170]. https://www.nice.org.uk/guidance/ cg170; Accessed 24 May 2020.

58. Howes, O. D. et al. Autism Spectrum Disorder: consensus guidelines on assessment, treatment and research from the British Association for Psychopharmacology. J. Psychopharmacol. Oxf. Engl. 32, 3-29 (2018).

59. Accordino, R. E., Kidd, C., Politte, L. C., Henry, C. A. \& McDougle, C. J. Psychopharmacological interventions in autism spectrum disorder. Expert Opin. Pharmacother. 17, 937-952 (2016).

60. Jobski, K., Höfer, J., Hoffmann, F. \& Bachmann, C. Use of psychotropic drugs in patients with autism spectrum disorders: a systematic review. Acta Psychiatr. Scand. 135, 8-28 (2017).

61. Cortese, S. et al. Comparative efficacy and tolerability of medications for attention-deficit hyperactivity disorder in children, adolescents, and adults: a systematic review and network metaanalysis. Lancet Psychiatry 5, 727-738 (2018). 
62. Hennissen, L. et al. Cardiovascular Effects of Stimulant and NonStimulant Medication for Children and Adolescents with ADHD: A Systematic Review and Meta-Analysis of Trials of Methylphenidate, Amphetamines and Atomoxetine. CNS Drugs 31, 199-215 (2017).

63. Nissen, S. E. ADHD drugs and cardiovascular risk. N. Engl. J. Med. 354, 1445-1448 (2006).

64. Silva, R. R., Skimming, J. W. \& Muniz, R. Cardiovascular safety of stimulant medications for pediatric attentiondeficit hyperactivity disorder. Clin. Pediatr. (Phila.) 49, 840-851 (2010).

65. Cooper, W. O. et al. ADHD Drugs and Serious Cardiovascular Events in Children and Young Adults. N. Engl. J. Med. 365, 1896-1904 (2011).

66. Dalsgaard, S., Kvist, A. P., Leckman, J. F., Nielsen, H. S. \& Simonsen, M. Cardiovascular Safety of Stimulants in Children with Attention-Deficit/ Hyperactivity Disorder: A Nationwide Prospective Cohort Study. J. Child Adolesc. Psychopharmacol. 24, 302-310 (2014).

67. Gould, M. S. et al. Sudden death and use of stimulant medications in youths. Am. J. Psychiatry 166, 992-1001 (2009).

68. Olfson, M. et al. Stimulants and Cardiovascular Events in Youth with Attention-Deficit/Hyperactivity Disorder. J. Am. Acad. Child Adolesc. Psychiatry 51, 147-156 (2012).

69. Shin, J.-Y., Roughead, E. E., Park, B.-J. \& Pratt, N. L. Cardiovascular safety of methylphenidate among children and young people with attention-deficit/ hyperactivity disorder (ADHD): nationwide self controlled case series study. BMJ 353, i2550 (2016).

70. Winterstein, A. G. et al. Cardiovascular safety of central nervous system stimulants in children and adolescents: population based cohort study. BMJ 345, e4627 (2012).
71. Lee, S.-H. et al. Use of antipsychotics increases the risk of fracture: a systematic review and meta-analysis. Osteoporos. Int. 28, 1167-1178 (2017).

72. Papola, D., Ostuzzi, G., Thabane, L., Guyatt, G. \& Barbui, C. Antipsychotic drug exposure and risk of fracture: a systematic review and meta-analysis of observational studies. Int. Clin. Psychopharmacol. 33, 181-196 (2018).

73. Voth, M., Lustenberger, T., Auner, B., Frank, J. \& Marzi, I. What injuries should we expect in the emergency room? Injury 48, 2119-2124 (2017).

74. Clark, E. M. The epidemiology of fractures in otherwise healthy children. Curr. Osteoporos. Rep. 12, 272-278 (2014).

75. Morris, M. W. J. \& Bell, M. J. The socioeconomical impact of paediatric fracture clinic appointments. Injury 37, 395-397 (2006).

76. Fraser, L.-A.et al. Falls and fractures with atypical antipsychotic medication use: a population-based cohort study. JAMA Intern. Med. 175, 450-452 (2015).

77. Calarge, C. A. et al. Possible mechanisms for the skeletal effects of antipsychotics in children and adolescents. Ther. Adv. Psychopharmacol. 3, 278-293 (2013).

78. Chen, C.-Y., Lane, H.-Y. \& Lin, C.-H. Effects of Antipsychotics on Bone Mineral Density in Patients with Schizophrenia: Gender Differences. Clin. Psychopharmacol. Neurosci. 14, 238-249 (2016).

79. Siafis, S., Tzachanis, D., Samara, M. \& Papazisis, G. Antipsychotic Drugs: From Receptor-binding Profiles to Metabolic Side Effects. Curr. Neuropharmacol. 16, 1210-1223 (2018).

80. Salomone, E. et al. Use of early intervention for young children with autism spectrum disorder across Europe. Autism Int. J. Res. Pract. 20, 233-249 (2016).

81. Bowker, A., D’Angelo, N. M., Hicks, R. \& Wells, K. Treatments for Autism: Parental Choices and Perceptions of Change. J. Autism Dev. Disord. 41, 1373-1382 (2011). 
82. Kelleher, K. J. \& Gardner, W. Out of Sight, Out of Mind - Behavioral and Developmental Care for Rural Children. N. Engl. J. Med. 376, 1301-1303 (2017).

83. Wang, L., Mandell, D. S., Lawer, L., Cidav, Z. \& Leslie, D. L. Healthcare service use and costs for autism spectrum disorder: a comparison between medicaid and private insurance. J. Autism Dev. Disord. 43, 1057-1064 (2013).

84. NCSL - National Conference of State Legislatures. Autism and Insurance Coverage | State Laws. https://www. ncsl.org/research/health/autism-andinsurance-coverage-state-laws.aspx (2018).

85. World Health Organization \& Dukes, M. N.

G. Drug utilization studies: methods and uses. (World Health Organization. Regional Office for Europe; https://apps.who.int/ iris/handle/10665/260517, 1993).

86. clinicaltrials.gov/. National Institutes of Health (NIH) | ClinicalTrials.gov. https:// clinicaltrials.gov/; Accessed 24 May 2020.

87. Anagnostou, E. Clinical trials in autism spectrum disorder: evidence, challenges and future directions. Curr. Opin. Neurol. 31, 119-125 (2018).

88. Hong, M. P. \& Erickson, C. A. Investigational drugs in early-stage clinical trials for autism spectrum disorder. Expert Opin. Investig. Drugs 28, 709-718 (2019).

89. NCTO4047355. Propranolol for Challenging Behaviors in Autism - Rutgers, The State University of New Jersey. https://clinicaltrials. gov/ct2/show/NCTO4047355? recrs $=$ ad\&type $=$ Intr\&cond $=$ Autism\&intr $=$ Drug $\&$ phase $=12 \& d r a w=2 \&$ rank $=6$; Accessed 24 May 2020.

90. NCT03715153. Efficacy and Safety of Bumetanide Oral Liquid Formulation in Children Aged From 2 to Less Than 7 Years Old With Autism Spectrum Disorder Institut de Recherches Internationales Servier. https://clinicaltrials.gov/ ct $2 /$ showNCT03715153? recrs = ad\&type $=$ Intr\&cond $=$ Autism\&intr $=$ Drug\&phase $=12 \&$ draw $=3 \&$ rank $=10$; Accessed 24 May 2020.
91. Barthélémy, C. et al. Validation of the Revised Behavior Summarized Evaluation Scale. J. Autism Dev. Disord. 27, 139-153 (1997).

92. Freeman, B. J., Ritvo, E. R., Yokota, A. \& Ritvo, A. A Scale for Rating Symptoms of Patients with the Syndrome of Autism in Real Life Settings. J. Am. Acad. Child Psychiatry 25, 130-136 (1986).

93. Constantino, J. N. \& Gruber, C. P. Social responsiveness scale (SRS). (Torrance, CA; Western Psychological Services., 2012).

94. Mirenda, P. et al. Validating the Repetitive Behavior Scale-revised in young children with autism spectrum disorder. J. Autism Dev. Disord. 40, 1521-1530 (2010).

95. Perry, A. \& Factor, D. C. Psychometric validity and clinical usefulness of the Vineland Adaptive Behavior Scales and the AAMD Adaptive Behavior Scale for an autistic sample. J. Autism Dev. Disord. 19, 41-55 (1989).

96. Sparrow, S. S., Cicchetti, D. V.\&Saulnier, C. A. Vineland Adaptive Behavior Scales, Third Edition. (Bloomington, MN; Pearson's Clinical Assessment group, 2016).

97. Schneeweiss, S. Developments in postmarketing comparative effectiveness research. Clin. Pharmacol. Ther. 82, 143156 (2007).

98. Mitchell, A. J. \& Selmes, T. Why don't patients take their medicine? Reasons and solutions in psychiatry. Adv. Psychiatr. Treat. 13, 336-346 (2007).

99. Osterberg, L. \& Blaschke, T. Adherence to Medication. N. Engl. J. Med. 353, 487497 (2005).

100. Herrett, E. et al. Data Resource Profile: Clinical Practice Research Datalink (CPRD). Int. J. Epidemiol. 44, 827-836 (2015).

101. Unigwe, S. et al. GPs' confidence in caring for their patients on the autism spectrum: an online self-report study. Br. J. Gen. Pract. J. R. Coll. Gen. Pract. 67, e445-e452 (2017).

102. CPRD. Clinical Practice Research Datalink. CPRD: UK data driving realworld evidence https://cprd.com/home; Accessed 22 May 2020. 
103. Hagberg, K. W. \& Jick, S. S. Validation of autism spectrum disorder diagnoses recorded in the Clinical Practice Research Datalink, 1990-2014. Clin. Epidemiol. 9, 475-482 (2017).

104. Smeeth, L. et al. MMR vaccination and pervasive developmental disorders: a case-control study. Lancet Lond. Engl. 364, 963-969 (2004).

105. IBM Watson Health. White paper: IBM MarketScan Research Databases for Health Services Researchers. https://www.ibm.com/downloads/ cas/6KNYVVQ2; Accessed 01 May 2020.

106. SPARK Consortium. SPARK: A US Cohort of 50,000 Families to Accelerate Autism Research. Neuron 97, 488-493 (2018).

107. Fombonne, E. et al. Psychiatric and Medical Profiles of Autistic Adults in the SPARK Cohort. J. Autism Dev. Disord. (2020) doi:10.1007/s10803-02004414-6.

108. Feliciano, P. et al. Exome sequencing of 457 autism families recruited online provides evidence for autism risk genes. NPJ Genomic Med. 4, 19 (2019).

109. Fombonne, E., Goin-Kochel, R. P., O'Roak, B. J. \& SPARK Consortium. Beliefs in vaccine as causes of autism among SPARK cohort caregivers. Vaccine 38, 1794-1803 (2020).

110. Bhat, A. N. Is Motor Impairment in Autism Spectrum Disorder Distinct From Developmental Coordination Disorder? A Report From the SPARK Study. Phys. Ther. 100, 633-644 (2020). 



\section{Chapter 2}

Treatment utilisation in autism spectrum disorder 


\section{Chapter 2.1}

Psychiatric comorbidities and use of psychotropic medications in people with autism spectrum disorder in the United States
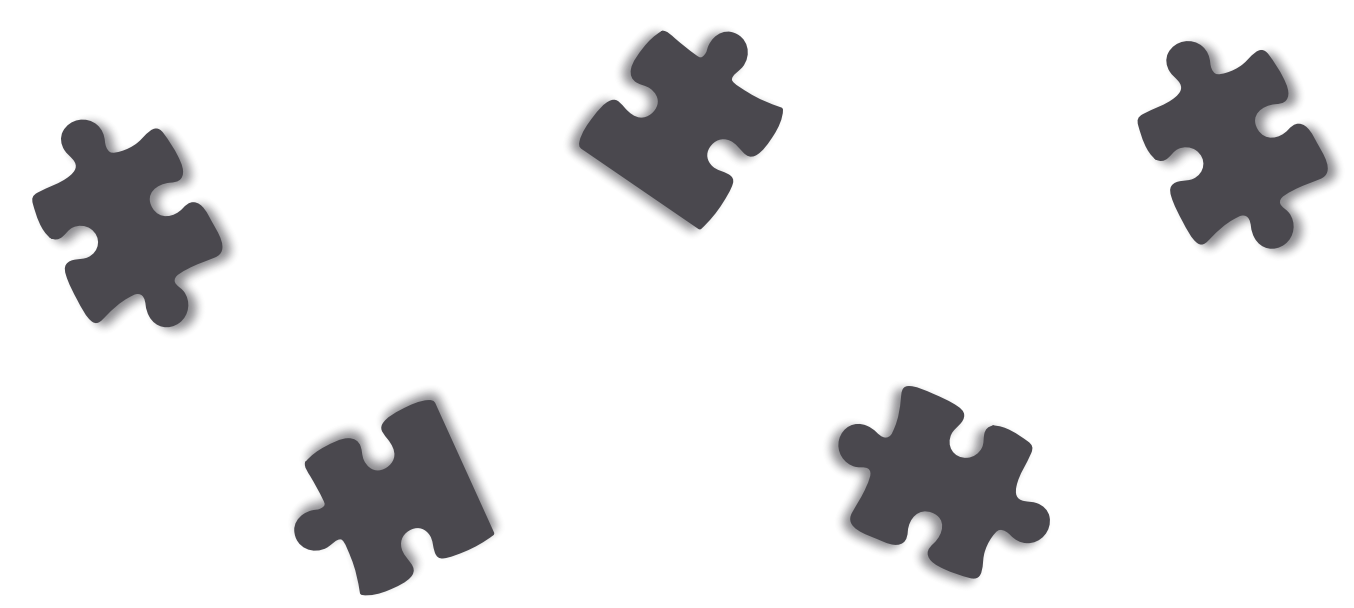


\begin{abstract}
This study investigated psychotropic medication usage in two large cohorts of people with autism spectrum disorder (ASD) throughout the calendar year 2014. The cohorts referred to individuals with commercial (employer-sponsored) and Medicaid insurance in the United States. We aimed to understand prescribing patterns of such medications across a wide age-range and in the presence/absence of other clinical and non-clinical characteristics, including psychiatric comorbidities. We described the prevalence and length of prescriptions by age, psychiatric comorbidity and overall. We also fitted multivariable logistic regression models to describe the relationship between treatments and subject characteristics simultaneously. Eighty percent of the identified population was male, although gender did not impact the odds of receiving medication. Medication use was strongly associated with age, increasing most rapidly before adulthood; generally plateauing thereafter. All psychiatric comorbidities studied also individually increased the chances of medication use, with epilepsy and ADHD having the highest associations in both the commercial (odds ratio $(O R)>7$ ) and Medicaid (OR around 12) cohorts. Those in non-capitated insurance plans, in foster care and white individuals also had increased odds of prescriptions. Overall, slightly more Medicaid enrolees received any psychotropic treatment (commercial: 64\%, Medicaid: 69\%). Nonetheless in both cohorts, a large proportion of individuals received treatment even without a diagnosis of any other psychiatric comorbidity (commercial: 31\%, Medicaid: 33\%). In summary, this report sheds new light on the latest patterns of psychiatric comorbidity profile and psychopharmacological treatment patterns in ASD.
\end{abstract}

\title{
Lay summary
}

This study identified a large number of children and adults in the US with autism spectrum disorder (autism) from employer-sponsored and government funded (Medicaid) health insurance data. Psychotropic medications were used by over two thirds of people, and four in ten people received two medications at the same time. The chances of receiving medication increased for individuals with other psychiatric conditions (e.g. ADHD), and also increased with age. 


\section{Introduction}

The personal, family and public health impact of autism spectrum disorder (ASD) is considerable. The latest reported prevalence estimates of ASD in the United States (US) were $2.24 \%{ }^{1}, 1.46 \%{ }^{2}$, and $2 \%^{3}$ in the National Health Interview Survey, Autism and Developmental Disabilities Monitoring Network, and National Survey of Children's Health, respectively. Estimates also suggest that the prevalence of ASD has been increasing over the last two decades ${ }^{4-6}$.

ASD has a documented impact on affected individuals and their families, for example individuals with an ASD diagnosis experience increased mortality compared with the general population ${ }^{7}$. Other psychiatric conditions are more commonly diagnosed in individuals with ASD than in typically developing individuals, in both adult and paediatric populations ${ }^{8-11}$. For example, in one of the largest studies, Abdallah et al ${ }^{11}$ found that $73 \%$ of individuals with ASD had at least one other psychiatric diagnosis, with the most common being attention deficit hyperactivity disorder (ADHD, 7.9\%), and depression (6.8\%). In another study, 71\% of children with ASD had at least one other DSM-IV psychiatric comorbidity diagnosis, with social anxiety disorder, ADHD and oppositional defiant disorder being the most common ${ }^{8}$. Children with ASD incur six-fold higher medical costs than children without ASD and costs have been found to increase in the presence of another neurodevelopmental disorder (e.g. children with intellectual disability have much higher costs than children with ASD alone) ${ }^{12}$.

Pharmacological treatment options for ASD are currently limited. Only two drugs have been approved in the US by the Food and Drug Administration (FDA) for the treatment of ASD: risperidone in 2006 and aripiprazole in 2009. Both drugs are atypical antipsychotics and are approved for the treatment of irritability associated with ASD. Neither targets the core symptoms of ASD and both are associated with important adverse events, such as tardive dyskinesia, weight gain and sedation. Psychotropic medications are extensively used to treat comorbid psychiatric morbidities, to improve ASD-associated symptoms, and off-label (non-evidence-based) to treat ASD's core symptoms ${ }^{13}$. For example, in a Medicaid dataset of children and adolescents with ASD, Schubart et al ${ }^{14}$ found that approximately $65 \%$ of individuals received at least one psychotropic medication and that psychotropic prescribing had increased over 2000 to 2003.

Earlier studies of ASD treatment patterns in claims used datasets from more than a decade ago, limited their analyses to either commercial or Medicaid insured individuals, and/or mostly focused on one age group (mainly children and adolescents). We wished to better understand psychiatric comorbidities, to describe recent patterns in psychotropic and antiepileptic medication prescribing in people with ASD of all ages, and to identify characteristics associated with use of these prescription medications. For this, we analysed two populations in the US: one of people with ASD insured via commercial providers and another insured via the government's Medicaid program. 


\section{Methods}

\section{Data sources and study population}

This study used US administrative insurance claims data from the Truven Health MarketScan ${ }^{\circledR}$ Commercial Database (abbreviated as "commercial") and the Truven Health MarketScan Multi-State Medicaid Database (abbreviated as "Medicaid"). The commercial database contains data from active employees, early retirees, Consolidated Omnibus Budget Reconciliation Act (COBRA) continuees, and dependents insured by employer-sponsored plans, while the Medicaid database contains data from government funded health insurance enrolees, who typically qualify due to low income or disability. The commercial database covers all states in the US and the Medicaid database covers approximately 10-12 states. The data provider cannot disclose which exactly states these are, but assure they are geographically distributed across the US. Both datasets include insurance claims across the continuum of care (e.g. inpatients, outpatient, outpatient pharmacy, carve-out behavioural healthcare etc.). Research using the MarketScan data has been widely published in peer-reviewed medical and health services journals ${ }^{15}$ and these data are fully anonymised and comply with the US Health Insurance Portability and Accountability Act (HIPAA). The commercial dataset is representative of employees and dependents (mainly children in the case of this study) of mid to large sized companies, with company-sponsored medical insurance. The Medicaid dataset is representative of lower income families and persons with certain disabilities.

The study period was the calendar year 2014. Enrolees were eligible for the analysis if they had at least two claims for ASD (International Classification of Diseases, Ninth Revision (ICD-9) codes 299.0x, 299.8x, 299.9x), of which at least one had to be recorded in 2014 (sample size: commercial: 87,297, Medicaid: 74,744). Requiring at least two codes has been shown to be appropriate in claims analysis to identify ASD ${ }^{16}$. Enrolees below the age of 3 were excluded given diagnostic uncertainties ${ }^{17}$, as were people with any Rett's syndrome claim ${ }^{18,16}$. Finally, all participants were required to have full medical and drug coverage for the whole of 2014, as well as in November and December of 2013 in order to capture drugs dispensed in 2013 with enough quantity supplied to last into 2014. This resulted in two ASD cohorts of 46,943 commercially-insured and 46,696 Medicaid-insured subjects.

\section{Definition of study variables}

Prescription records (mail-order or card program prescription drug claim) for the following psychotropic medication classes were collected: stimulants, antidepressants, antipsychotics/tranquilizers, hypotensive agents, anticonvulsants and anxiolytics/ sedatives/ hypnotics. Psychiatric comorbidities (medical claims) of interest were: attention deficit hyperactivity disorder (ADHD), anxiety disorder, bipolar disorder, conduct disorder, depression, epilepsy, intellectual disability, schizophrenia and sleep disturbances. Drug classes were identified using medication classes from the Truven Health Red Book and 
comorbidities using ICD-9 codes (see online supplementary material for full code lists; https://doi.org/10.1002/aur.1848). We defined psychotropic polypharmacy as having a days-supply for at least two drugs in different medication classes overlapping by 30 consecutive days or more.

Demographic variables available in both datasets were age, sex and insurance plan type. We grouped the plan types based on whether they contained capitation or not. Geographic region was available for the commercially insured cohort only, and race was available for Medicaid only. Also in Medicaid only, we extracted information from the "basis of eligibility" information to derive whether an individual was in foster care, given its association with psychotropic medication use in ASD ${ }^{19}$. ASD subtype was defined by the subjects' most frequent ICD-9 code during 2014 (299.0x=autistic disorder, 299.8x=other specified pervasive developmental disorders, 299.9x=unspecified pervasive developmental disorder).

\section{Data Analysis}

Each cohort was analysed separately. We described all demographic variables by absolute number and percentage, stratifying age (in years) into the following groups: 3-4, 5-11, 12-17, 18-24, 25-49 and $\geq 50$. In bivariate analyses, we described the percentage of subjects with different comorbidities and psychotropic medications by age group and overall. The number of days on different treatments in 2014 (maximum 365 days) was described by means and standard deviations. The prevalence of each medication was also reported within subgroups of subjects where each of the psychiatric comorbidities was observed.

To identify characteristics associated with psychotropic prescribing patterns, we fitted multivariable logistic regression models for the outcomes "any psychotropic medication" and "psychotropic polypharmacy" separately. An odds ratio (OR) greater than one indicates an increased likelihood of the variable to be associated with psychotropic medication use. We included all psychiatric comorbidities, insurance type and demographic variables as covariates. Covariate reduction techniques and interactions were not applied. We assessed model fit using adjusted $r$-squared values for generalised linear models, the area under the curve (AUC) scores, and both Pearson and deviance residuals ${ }^{20}$. The Hosmer and Lemeshow statistic for goodnessof-fit was not employed, as the probability of rejecting an appropriate model increases with sample size, and is actually unsuitable in sample sizes greater than 25,000; like in this case ${ }^{21}$.

\section{Results}

In both cohorts, people with ASD were four times more likely to be male and more than two thirds were between the age of 5 and 17 years (Table 2.1.1). Autistic disorder (ICD-9 299.0x) was the predominant ASD diagnosis code. The commercially insured sample was geographically balanced across the US. The most frequent psychiatric comorbidity was ADHD (around 40\%), followed by anxiety disorder (commercial) or intellectual disability (Medicaid). 
Table 2.1.1: Description of ASD cohorts in commercial and Medicaid claims

\begin{tabular}{|c|c|c|}
\hline \multirow[t]{2}{*}{ Characteristic } & \multicolumn{2}{|c|}{ ASD Cohort } \\
\hline & Commercial & Medicaid \\
\hline $\mathrm{N}$ & 46,943 & 46,696 \\
\hline \multicolumn{3}{|l|}{ Gender [n/\%] } \\
\hline Male & $37,775(80.47)$ & $37,116(79.48)$ \\
\hline Female & $9,168(19.53)$ & $9,580(20.52)$ \\
\hline \multicolumn{3}{|l|}{ Age (years)[n/\%] } \\
\hline $3-4$ & $2,959(6.30)$ & $3,440(7.37)$ \\
\hline $5-11$ & $17,420(37.11)$ & $19,527(41.82)$ \\
\hline $12-17$ & $15,086(32.14)$ & $12,954(27.74)$ \\
\hline $18-24$ & $8,370(17.83)$ & $6,716(14.38)$ \\
\hline $25-49$ & $2,722(5.80)$ & $3,807(8.15)$ \\
\hline$\geq 50$ & $386(0.82)$ & $252(0.54)$ \\
\hline \multicolumn{3}{|l|}{ Geographic region [n/\%] } \\
\hline Northeast & $11,508(24.51)$ & N/A \\
\hline North Central & $10,909(23.24)$ & $\mathrm{N} / \mathrm{A}$ \\
\hline South & $13,660(29.10)$ & $\mathrm{N} / \mathrm{A}$ \\
\hline West & $9,171(19.54)$ & $N / A$ \\
\hline Unknown & $1,695(3.61)$ & N/A \\
\hline \multicolumn{3}{|l|}{ Race [n/\%] } \\
\hline White & N/A & $23,404(50.12)$ \\
\hline Black & $\mathrm{N} / \mathrm{A}$ & $8,792(18.83)$ \\
\hline Hispanic & N/A & 1,909 (4.09) \\
\hline Other & $\mathrm{N} / \mathrm{A}$ & $12,591(26.96)$ \\
\hline \multicolumn{3}{|l|}{ Most prevalent ASD diagnosis [n/\%] } \\
\hline Autistic disorder & $30,588(65.16)$ & $35,554(76.14)$ \\
\hline Other specified pervasive developmental disorder & $13,952(29.72)$ & $9,567(20.49)$ \\
\hline Unspecified pervasive developmental disorder & $2,403(5.12)$ & $1,575(3.37)$ \\
\hline \multicolumn{3}{|l|}{ Insurance plan type (grouped) [n/\%] } \\
\hline Capitated Plan & $6,015(12.81)$ & $17,276(37.00)$ \\
\hline Non-Capitated Plan & $40,432(86.13)$ & $29,404(62.97)$ \\
\hline Missing/Unknown & $496(1.06)$ & $16(0.03)$ \\
\hline \multicolumn{3}{|l|}{ Selected psychiatric comorbidities [n/\%] } \\
\hline 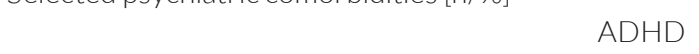 & $17,756(37.82)$ & $18,905(40.49)$ \\
\hline Anxiety disorder & $11,614(24.74)$ & $6,526(13.98)$ \\
\hline Bipolar disorder & $2,298(4.90)$ & $3,718(7.96)$ \\
\hline Conduct disorder & $5,547(11.82)$ & $9,031(19.34)$ \\
\hline Depression & $4,764(10.15)$ & $4,290(9.19)$ \\
\hline Epilepsy & $3,335(7.10)$ & $5,023(10.76)$ \\
\hline Intellectual disability & $2,362(5.03)$ & $10,910(23.36)$ \\
\hline Schizophrenia & $514(1.09)$ & $1,070(2.29)$ \\
\hline Sleep disturbances & $2,688(5.73)$ & $4,400(9.42)$ \\
\hline None & $17,299(36.85)$ & $13,403(28.70)$ \\
\hline \multicolumn{3}{|l|}{ Selected psychotropic medications by class [n/\%] } \\
\hline Anticonvulsants & $7,968(16.97)$ & $11,550(24.73)$ \\
\hline Antidepressant & $15,636(33.31)$ & $13,380(28.65)$ \\
\hline Antipsychotics/Tranquilizer & $11,801(25.14)$ & $16,138(34.56)$ \\
\hline
\end{tabular}


Table 2.1.1: Description of ASD cohorts in commercial and Medicaid claims (cont.)

\begin{tabular}{|c|c|c|}
\hline \multirow[t]{2}{*}{ Characteristic } & \multicolumn{2}{|c|}{ ASD Cohort } \\
\hline & Commercial & Medicaid \\
\hline \multicolumn{3}{|l|}{ Selected psychotropic medications by class [n/\%] } \\
\hline Anxiolytics/Sedatives/Hypnotics & $5,961(12.70)$ & $8,256(17.68)$ \\
\hline Hypotensive agents & $9,312(19.84)$ & $14,626(31.32)$ \\
\hline Stimulants & $14,051(29.93)$ & $14,924(31.96)$ \\
\hline None & $17,043(36.31)$ & $14,494(31.04)$ \\
\hline \multicolumn{3}{|l|}{ Basis of eligibility is foster care [n/\%] } \\
\hline Yes & $\mathrm{N} / \mathrm{A}$ & $1,342(2.87)$ \\
\hline No & $\mathrm{N} / \mathrm{A}$ & $45,354(97.13)$ \\
\hline
\end{tabular}

ASD=Autism Spectrum Disorder, ADHD=Attention Deficit Hyperactivity Disorder. N/A=Not Applicable.

Having none of the selected psychiatric comorbidities was more common in the commercial cohort (37\%) than Medicaid (29\%). The largest difference in comorbidity profiles between the databases was that Medicaid had a far larger proportion of people with intellectual disability across all age groups (commercial: 5\%, Medicaid: 23\%; see Table 2.1.2a and 2.1.2b).

Thirty-six percent of individuals in the commercial cohort were not receiving any psychotropic medication, compared to 31\% in Medicaid. Antidepressants (33\%) and stimulants (30\%) were the most commonly prescribed drug classes in the commercial cohort. Antidepressant usage was lower (29\%) and stimulant usage was similar (32\%) in the Medicaid cohort. Medicaid had a markedly higher proportion of individuals prescribed with each of the other four drug classes, with antipsychotics/tranquilizer (35\%) being the most common.

In the commercial-claims cohort, 77\% in the 3-4 years age group had an ASD diagnosis only, without other psychiatric comorbidities; this proportion was only 53\% in Medicaid (Table 2.1.2a and 2.1.2b). Furthermore, in both cohorts, the frequencies of different comorbidities increased with age, at least until adulthood. In commercial claims, psychotropic medication use in children aged 3-4 years was below 5\% for each of the six classes investigated (Table 2.1.2a). In all children in this age group, only $11 \%$ were prescribed a psychotropic medication; however, during adolescence (12-17 years), this proportion increased to $76 \%$. In the Medicaid data, 25\% of children in the 3-4 years age group were prescribed at least one psychotropic medication (Table 2.1.2b). The most commonly prescribed medication in this group was a 2 adrenergic agonists (clonidine and guanfacine, 14\%). The proportion of Medicaid adolescents with at least one psychiatric medication was 80\%. In both cohorts, most drug classes studied, except Anxiolytics/ Sedatives/Hypnotics, were taken for more than seven months of the year. Anticonvulsants and antipsychotics/tranquilizers were associated with the highest number of days treated; taken about two-thirds of the year on average. Generally, the number of days treated increased with age, and increased more profoundly for antipsychotics/tranquilizers. 


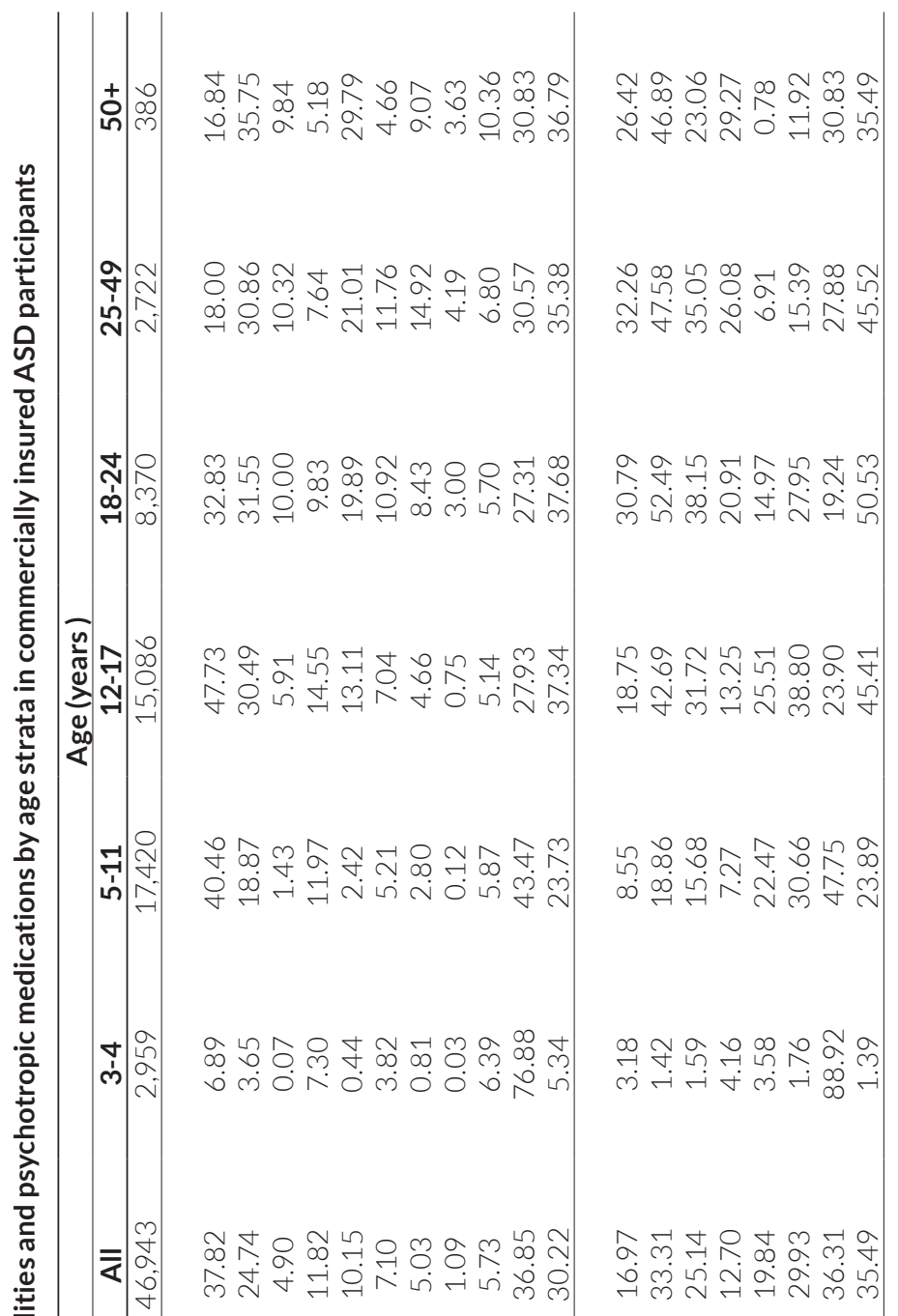

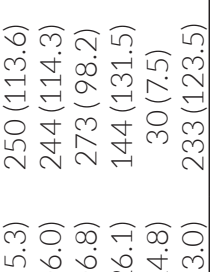

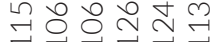
$\exists=\exists \exists=$ $\infty m \infty \infty$ ํํㄹำ

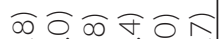
๑ $\exists=\exists=\exists$ 过 슘ํำ N N $\mathrm{N} N$

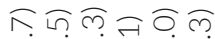
ปี่ $\exists=\underset{y}{-1}=\sqrt{-1}=$

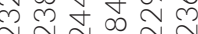

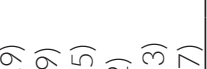
จ

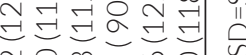
$\approx \circ \cdots a$ 츷ํํㄱำ

ฝฺ즈ำ

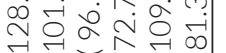
즌ำㄴำ oิ $\widehat{\alpha} \widehat{a}$ 걱요 ले $\exists= \pm \exists=$

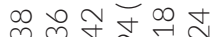

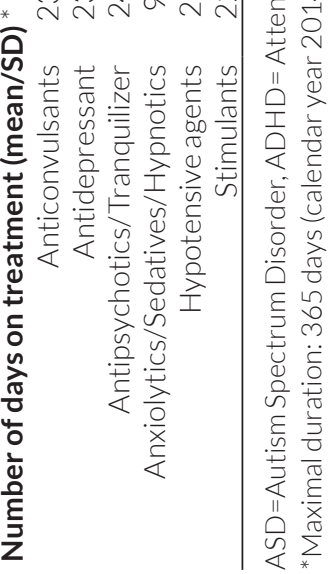

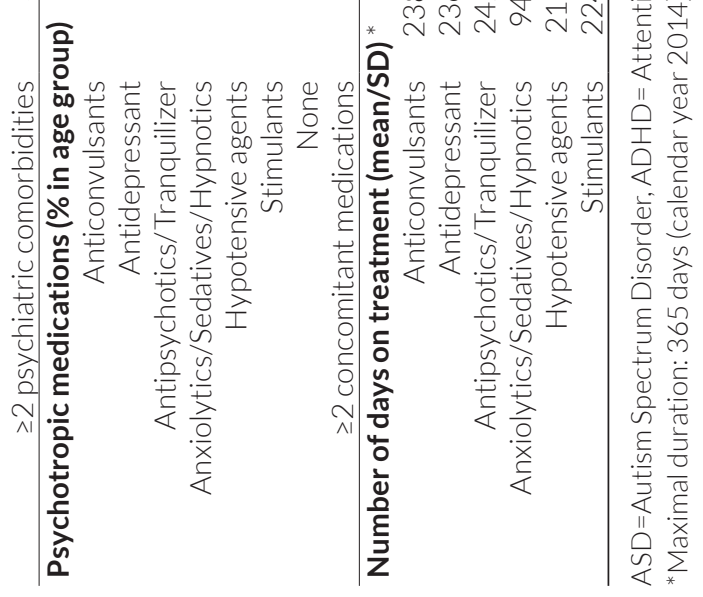




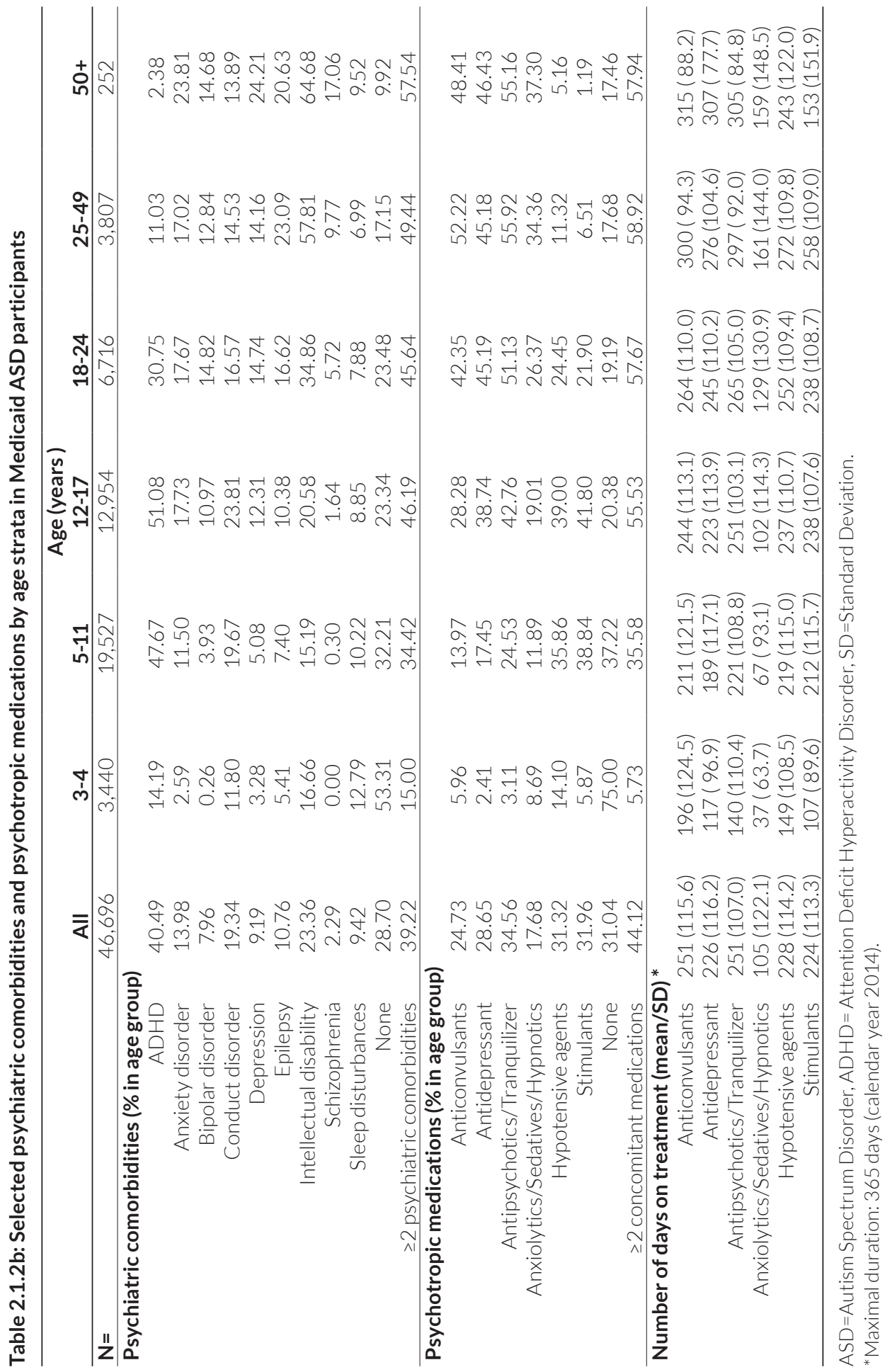


Table 2.1.3 summarises the results of the logistic regressions. In the commercial cohort, age was associated with receiving any psychotropic medication and with polypharmacy, with the highest odds at age 18-24 years (OR 21 for any medication, OR 38 for polypharmacy). This pattern was also observed in Medicaid, but with the highest odds in the age group 25-49 years (OR 12 for any medication, OR 20 for polypharmacy). In both cohorts, gender was not associated with prescribing. All psychiatric comorbidities were associated with prescribing, with epilepsy and ADHD having the highest associations in both the commercial $(O R>7)$ and Medicaid (OR around 12) cohorts. Patients with schizophrenia were more likely to have received psychotropic medication if they were covered by Medicaid compared to individuals covered by commercial insurance. In both cohorts, participating in a capitated plan decreased the likelihood of prescribing. In Medicaid, white individuals and those in foster care also had increased odds of psychotropic prescriptions. All four models had good predictive ability (AUCs between 0.79 and 0.88). Residual checking and adjusted $r$-squared values (between 0.32 and 0.50) indicated that the models fitted the data well in general terms.

In both cohorts, people with ASD were most likely to receive polypharmacy if they had a concomitant diagnosis code of bipolar disorder or schizophrenia (Figure 2.1.1). Over $30 \%$ of participants received medication in the absence of a coded psychiatric condition other than ASD; one sixth in this group had polypharmacy prescribing. Overall, however, medications were most frequently prescribed in conjunction with the indicated psychiatric condition (Figure 2.1.2).

Finally, Figure 2.1.3 shows the mean number of overlapping psychotropic treatments as a function of age for the commercial and Medicaid cohorts. The number of concomitant drug classes increased from an early age, plateauing during adolescence. In older individuals, and especially in the commercial cohort, a small reduction in the number of overlapping drug classes was observed, although the number of subjects at older ages is small, making estimates less certain. 


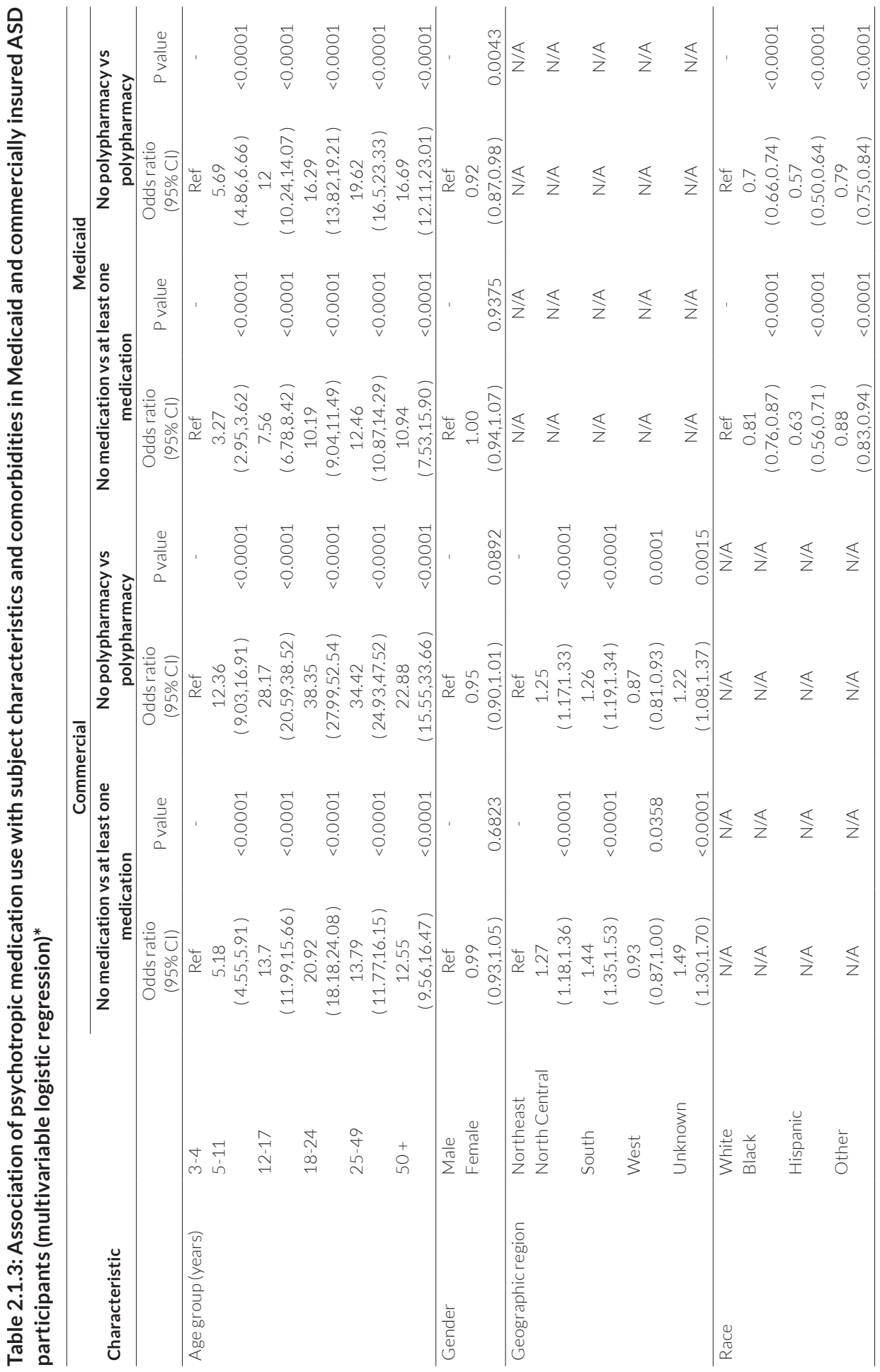




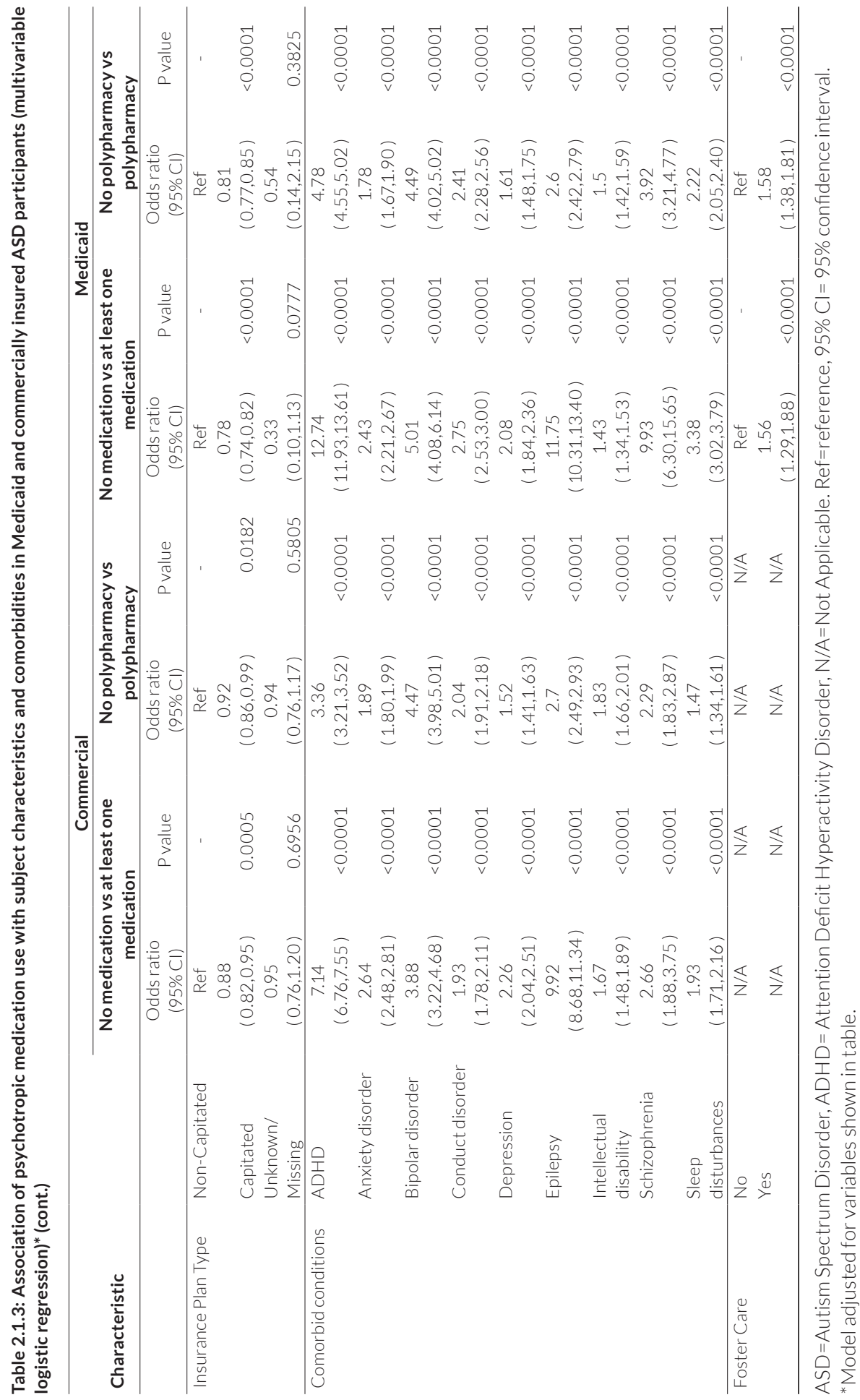


Figure 2.1.1: Psychotropic medication use (any and polypharmacy) by comorbidity

\section{Commercial}

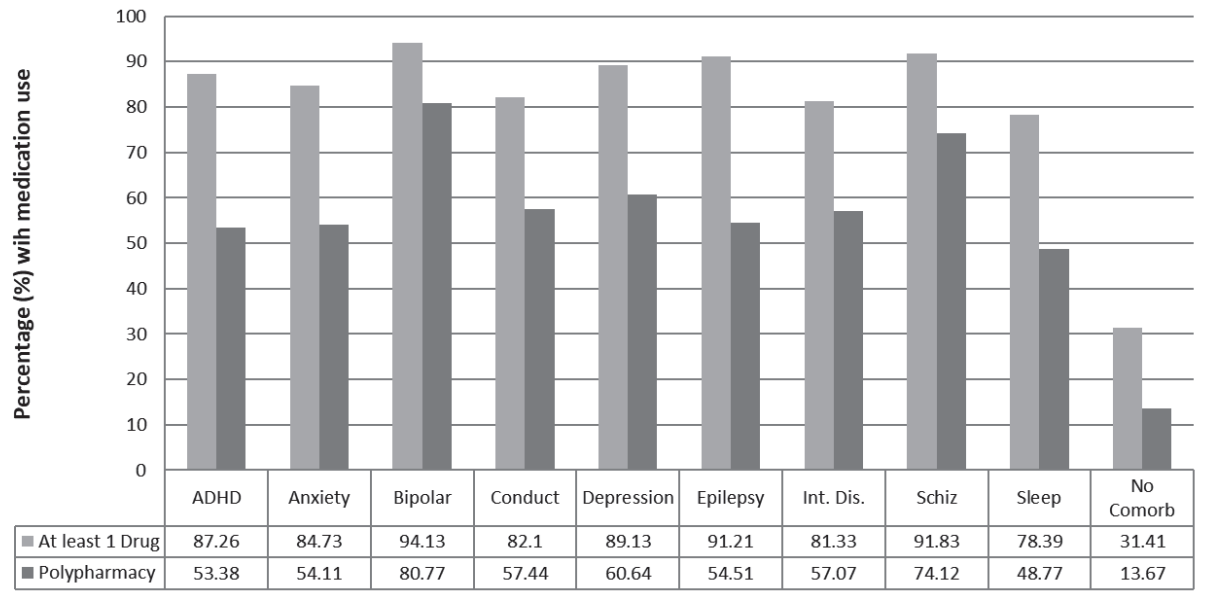

\section{Medicaid}

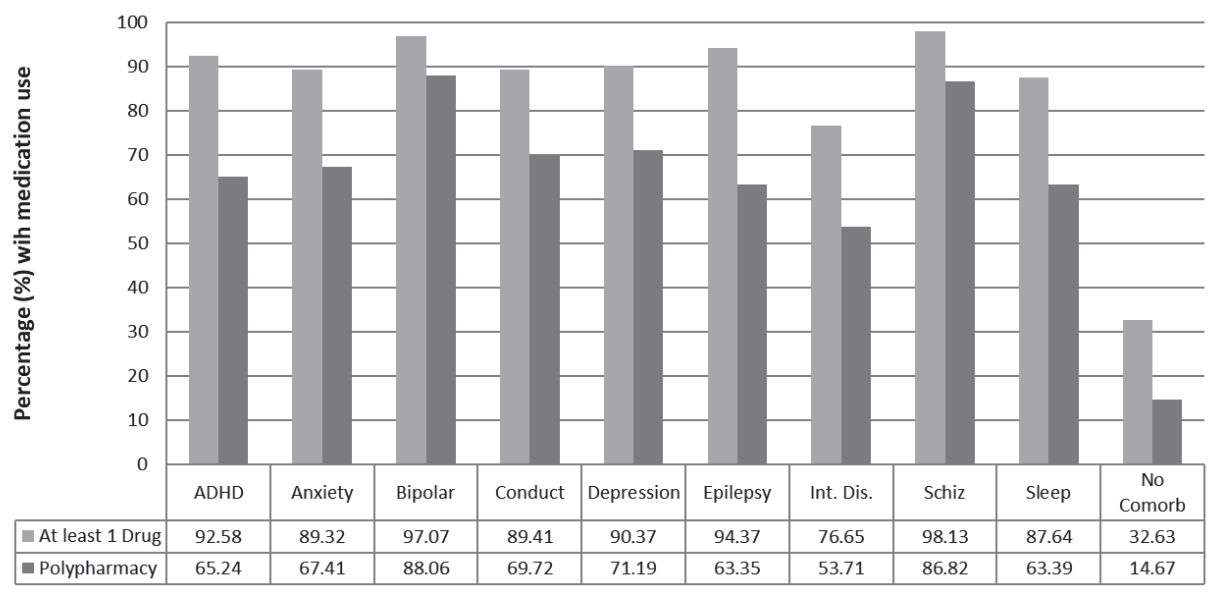

ASD =Autism Spectrum Disorder, ADHD= Attention Deficit Hyperactivity Disorder, Int. Dis= Intellectual Disability, No Comorb=No Comorbidity, Schiz= Schizophrenia. 
Figure 2.1.2: Cross-tabulation of psychiatric comorbidities with classes of psychotropic medications

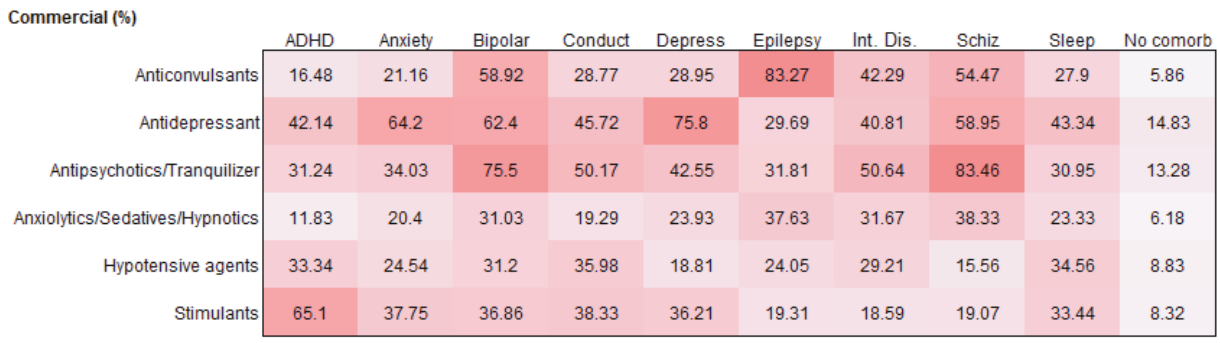

\begin{tabular}{|c|c|c|c|c|c|c|c|c|c|c|}
\hline \multicolumn{11}{|l|}{ Medicaid (\%) } \\
\hline & $\mathrm{ADHD}$ & Anxiety & Bipolar & Conduct & Depress & Epilepsy & Int. Dis. & Schiz & Sleep & No comorb \\
\hline Anticonvulsants & 25.48 & 33.14 & 63.07 & 37.54 & 40.37 & 86.68 & 39.89 & 66.26 & 31.57 & 7.19 \\
\hline Antidepressant & 37.57 & 61.02 & 59.31 & 42.8 & 63.33 & 30.28 & 33.72 & 62.8 & 42.16 & 11.62 \\
\hline Antipsychotics/Tranquilizer & 44.05 & 48.71 & 83.62 & 59.93 & 56.55 & 42.5 & 47.31 & 93.55 & 39.59 & 15.47 \\
\hline Anxiolytics/Sedatives/Hypnotics & 17.18 & 28.75 & 32.97 & 23.53 & 27.27 & 44.18 & 26.48 & 43.74 & 25.2 & 8.62 \\
\hline Hypotensive agents & 51.82 & 39.67 & 48.17 & 51.52 & 37.55 & 32.43 & 32.42 & 26.92 & 55.95 & 12.21 \\
\hline Stimulants & 68.15 & 40.97 & 43.28 & 47.58 & 39.91 & 20.43 & 21.5 & 20.56 & 43.86 & 7.64 \\
\hline
\end{tabular}

Legend

$0 \%$ $100 \%$

Percentages are calculated with psychiatric comorbidity as the denominators. Legend provided to help find associations. ASD=Autism Spectrum Disorder, ADHD= Attention Deficit Hyperactivity Disorder, Depress=Depression, Int. Dis=Intellectual Disability, No Comorb=No Comorbidity, Schiz= Schizophrenia.

Figure 2.1.3: Number of overlapping psychotropic medications classes prescribed by year of age
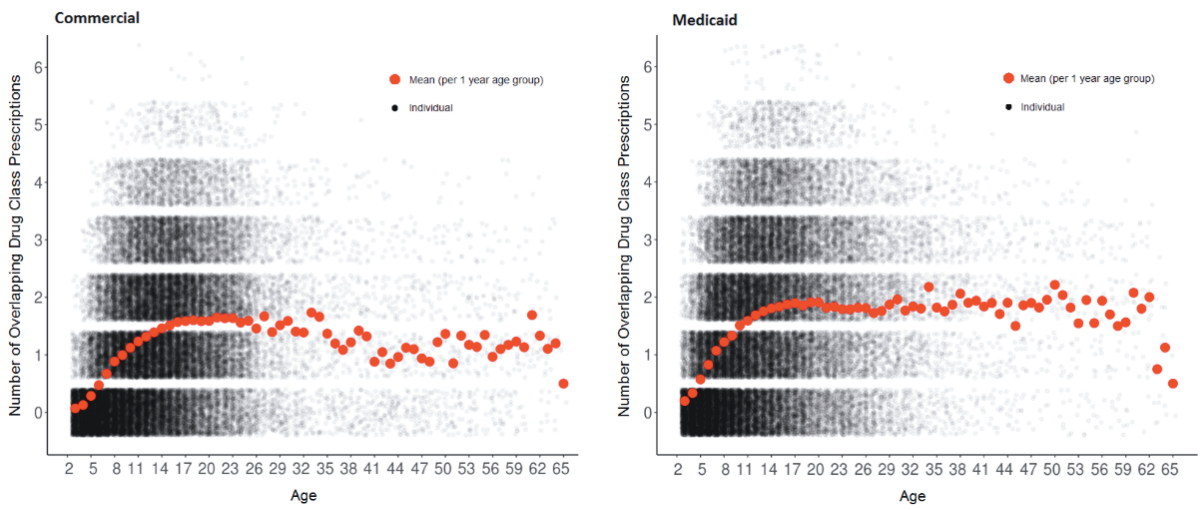

Individual observations are scattered slightly around the exact coordinate, such that they do not overlap. Age given in years. 


\section{Discussion}

We found that psychiatric comorbidities and psychotropic medication use are common in individuals with ASD in the US and, further, that psychotropic medications were prescribed in individuals with ASD without coded comorbid psychiatric conditions, potentially suggesting their use to treat symptoms of ASD. If so, the high prescribing rates may indicate a high symptom burden and large unmet medical need, as ASD is a non-labelled indication for almost all drug classes studied. Our regression models also identified characteristics associated with psychotropic prescribing, such as age, psychiatric comorbidities, and type of insurance plan.

Overall, the pattern of results from the commercial and Medicaid cohorts were similar. However, compared to the commercial cohort, a larger proportion of subjects in the Medicaid cohort had psychiatric comorbidity, used psychotropic medication, and had polypharmacy. This is consistent with the findings by Wang et al ${ }^{22}$ that Medicaid-covered individuals had higher total healthcare, ASD-specific, and psychotropic medication costs. This could be due to the different enrolment criteria in commercial and Medicaid insurance. Individuals with severe ASD are more likely to fulfil disability criteria in Medicaid waiver programs, which allow individuals to receive support in ASD treatment through Medicaid regardless of family income. This may have contributed to the larger proportion of intellectually disabled individuals in the Medicaid cohort. Another potential reason is that commercial insurance plans may not reimburse certain treatments for ASD, resulting in lower observed prescription rate in the database. We also found that non-capitated insurance plans were associated with psychotropic treatment. This could be due to the requirement for capitated plans to keep expenditure under a certain budget. It could also be because those who enrol in non-capitated plans are generally in more genuine need of treatment.

Our findings are largely consistent with earlier studies of ASD treatment patterns in claims, although this current study uses more recent data and has a larger and broader sample than previous work in data more than a decade old. Similar to the overall prescribing rate we found, Schubart et al ${ }^{14}$ found that $65 \%$ of children and adolescents used psychotropic medication in a Medicaid analysis of years 2000-2003. In a similar age group in calendar year 2001 and also in Medicaid, Mandell et al ${ }^{19}$ found that $56 \%$ used any psychotropic medication, with neuroleptics, antidepressants, and stimulants most frequently prescribed. They also showed that age and several psychiatric comorbidities were associated with psychotropic medication use in this age group, although they did not consider anticonvulsants in the study. Oswald and Sonenklar ${ }^{17}$ found increasing psychotropic medication use from 5 years and older in a study of people with ASD younger than 21 years using commercial claims for the year 2002, and Spencer et al ${ }^{18}$ reported that $64 \%$ of children and adolescents with ASD were using at least one psychotropic medication using commercial claims for the years 2001-2009. Also in commercial claims 
but for an adult ASD population only, Vohra et al ${ }^{23}$ showed that annually, around $85 \%$ of subjects were prescribed a psychotropic medication, between years 2000-2008.

Other studies on medication use in subjects with ASD have surveyed parents, with generally inferior sample size. For example, Martin et al ${ }^{24}$ investigated 109 subjects of whom 55\% were taking at least one psychotropic medication and 29\%, at least two concomitantly. About a third took antidepressants and one-fifth took stimulants. In two-thirds of individuals with ASD, anxiety-related symptoms were the main reason for prescribing. As in our study, Aman et al ${ }^{4}$ showed that psychotropic medication use was associated with age. In a web-based survey Rosenberg et al ${ }^{25}$ with over 5,000 parents of children with ASD in the US (conducted in 2008), 35\% used at least one psychotropic medication; this figure is considerably lower than ours and previous studies have found. This may be attributable to the way the cohort was selected and to the extent that parents were able to correctly provide prescription drug information. Also using parent reported information from 2007-2011 in the Autism Treatment Network (ATN) registry, Coury et al ${ }^{26}$ estimated that $27 \%$ of individuals with ASD aged $2-17$ years $(N=2,853)$ took one or more psychotropic medication. The study results agreed with ours in the respect that age and presence of psychiatric comorbidities increased the likelihood of medication. Finally, in a narrower age range of 13-17 years, Frazier et al ${ }^{27}$ also showed that an ADHD diagnosis in addition to ASD increased the frequency of psychotropic medication to 58\%, from $34 \%$ for those with ASD alone. In this case, the parent reported data was captured for 890 subjects via telephone interview and email questionnaire, in 2001.

In another study in the UK, electronic clinical records of children in secondary care between 2008 and 2013 were abstracted $(n=3,482)$. Only about 10\% received antipsychotics, which is substantially lower than that observed in the US. The use of other psychotropic medications was not reported in this publication ${ }^{28}$.

Our analysis extended this previous work in several ways. Firstly, it analysed up-to-date data using two contemporary cohorts and presented the commercially and Medicaidcovered populations side by side. Secondly, we included a broader range of psychotropic medications than previous studies and also analysed a wider age range, which gave results on treatment patterns in both children and adults with ASD. In fact, the cohorts had over 10,000 adult ASD participants in each, which allowed an analysis of how psychiatric comorbidities and prescribing patterns continued into adulthood and beyond. Another strength of using claims data is our large sample size and general representativeness of both privately and publicly insured individuals. The data are recorded by physicians, minimising issues such as recall and selection bias present in surveys.

Our study has some limitations. Diagnosis of ASD or any of the psychiatric comorbidities was based on ICD codes used for reimbursement purposes. The study would also have benefited from having more granular geographical information to understand differences 
at state level. Also, reasons for prescribing psychotropic medications cannot be assessed. Claims data do not contain any details on the severity of ASD or the predominant symptoms. Lastly, some people with ASD may have eligibility in both commercial insurance and Medicaid. However, the prescription rates should not be meaningfully affected, as reimbursement will only be provided by one of the coverage means. Given the nature of the data, we could not assess whether concomitant psychiatric diagnoses were coded to allow for reimbursed prescription medicines for ASD, e.g. for symptomatic treatment, or whether individuals with ASD suffer from other psychiatric comorbidities with need for medication. Only detailed psychiatric assessments would be able to provide those insights.

In conclusion, psychotropic medication use and polypharmacy was frequent in this study of commercially and Medicaid insured people with ASD. This is concerning, considering the paucity of evidence supporting psychotropic medications in individuals with ASD. Longterm effects of psychotropic medications, particularly for developing brains in children, are not well understood, but preclinical evidence provides reason for concern ${ }^{29}$. Overall, this study highlights a need for evidence-based standards of care for psychotropic medication use in ASD, preferably through controlled trials, to assess long-term effectiveness and safety in individuals with different combinations of psychiatric comorbidities 


\section{References}

1. Zablotsky, B., Black, L. I., Maenner, M. J., Schieve, L. A. \& Blumberg, S. J. Estimated Prevalence of Autism and Other Developmental Disabilities Following Questionnaire Changes in the 2014 National Health Interview Survey. NatI. Health Stat. Rep. 87, 1-20 (2015).

2. Christensen, D. L. et al. Prevalence and Characteristics of Autism Spectrum Disorder Among Children Aged 8 Years-Autism and Developmental Disabilities Monitoring Network, 11 Sites, United States, 2012. Morb. Mortal. Wkly. Rep. Surveill. Summ. Wash. DC 2002 65, 1-23 (2016).

3. Blumberg, S. J. et al. Changes in prevalence of parent-reported autism spectrum disorder in school-aged U.S. children: 2007 to 2011-2012. Natl. Health Stat. Rep. 1-11, 1 p following 11 (2013).

4. Aman, M. G., Lam, K. S. L. \& Bourgondien, M. E. V. Medication patterns in patients with autism: Temporal, regional, and demographic influences. J. Child Adolesc. Psychopharmacol. 15, 116-126 (2005).

5. Matson, J. L. \& Kozlowski, A. M. The increasing prevalence of autism spectrum disorders. Res. Autism Spectr. Disord. 5, 418-425 (2011).

6. Kroncke, A. P., Willard, M. \& Huckabee, H. What Is Autism? History and Foundations. In: Assessment of Autism Spectrum Disorder. 3-9, ISBN 978-3319-25504-0 (Springer International Publishing, 2016).

7. Hirvikoski, T.et al. Premature mortality in autism spectrum disorder. Br. J. Psychiatry J. Ment. Sci. 208, 232-238 (2016).

8. Simonoff, E. et al. Psychiatric disorders in children with autism spectrum disorders: prevalence, comorbidity, and associated factors in a population-derived sample. J. Am. Acad. Child Adolesc. Psychiatry 47, 921-929 (2008).
9. Joshi, G. et al. The heavy burden of psychiatric comorbidity in youth with autism spectrum disorders: a large comparative study of a psychiatrically referred population. J. Autism Dev. Disord. 40, 1361-1370 (2010).

10. Skokauskas, N. \& Gallagher, L. Psychosis, affective disorders and anxiety in autistic spectrum disorder: prevalence and nosological considerations. Psychopathology 43, 8-16 (2010).

11. Abdallah, M. W. et al. Psychiatric comorbidities in autism spectrum disorders: findings from a Danish Historic Birth Cohort. Eur. Child Adolesc. Psychiatry 20, 599-601 (2011).

12. Peacock, G., Amendah, D., Ouyang, L. \& Grosse, S. D. Autism spectrum disorders and health care expenditures: the effects of co-occurring conditions. J. Dev. Behav. Pediatr. JDBP 33, 2-8 (2012).

13. Accordino, R. E., Kidd, C., Politte, L. C., Henry, C. A. \& McDougle, C. J. Psychopharmacological interventions in autism spectrum disorder. Expert Opin. Pharmacother. 17, 937-952 (2016).

14. Schubart, J. R., Camacho, F. \& Leslie, D. Psychotropic medication trends among children and adolescents with autism spectrum disorder in the Medicaid program. Autism Int. J. Res. Pract. 18, 631-637 (2014).

15. Truven Health Analytics Inc. COMMERCIAL CLAIMS AND ENCOUNTERS - MEDICARE SUPPLEMENTAL (Data Year 2014 Edition). (2015).

16. Burke, J. P. et al. Does a claims diagnosis of autism mean a true case? Autism Int. J. Res. Pract. 18, 321-330 (2014).

17. Oswald, D. P.\&Sonenklar, N. A. Medication use among children with autism spectrum disorders. J. Child Adolesc. Psychopharmacol. 17, 348-355 (2007). 
18. Spencer, D. et al. Psychotropic medication use and polypharmacy in children with autism spectrum disorders. Pediatrics 132, 833-840 (2013).

19. Mandell, D. S. et al. Psychotropic medication use among Medicaid-enrolled children with autism spectrum disorders. Pediatrics 121, e441-448 (2008).

20. Nagelkerke, N. J. D. A note on a general definition of the coefficient of determination. Biometrika 78, 691-692 (1991).

21. Paul, P., Pennell, M. L. \& Lemeshow, S. Standardizing the power of the HosmerLemeshow goodness of fit test in large data sets. Stat. Med. 32, 67-80 (2013).

22. Wang, L., Mandell, D. S., Lawer, L., Cidav, Z. \& Leslie, D. L. Healthcare service use and costs for autism spectrum disorder: a comparison between medicaid and private insurance. J. Autism Dev. Disord. 43, 1057-1064 (2013).

23. Vohra, R. et al. Prescription Drug Use and Polypharmacy Among Medicaid-Enrolled Adults with Autism: A Retrospective Cross-Sectional Analysis. Drugs - Real World Outcomes 3, 409-425 (2016).

24. Martin, A., Scahill, L., Klin, A. \& Volkmar, F. R. Higher-functioning pervasive developmental disorders: rates and patterns of psychotropic drug use. J. Am. Acad. Child Adolesc. Psychiatry 38, 923-931 (1999).

25. Rosenberg, R. E. et al. Psychotropic medication use among children with autism spectrum disorders enrolled in a national registry, 2007-2008. J. Autism Dev. Disord. 40, 342-351 (2010).

26. Coury, D. L. et al. Use of Psychotropic Medication in Children and Adolescents With Autism Spectrum Disorders. Pediatrics 130, S69-S76 (2012).

27. Frazier, T. W. et al. Prevalence and correlates of psychotropic medication use in adolescents with an autism spectrum disorder with and without caregiver-reported attention-deficit/ hyperactivity disorder. J. Child Adolesc. Psychopharmacol. 21, 571-579 (2011).
28. Downs, J. et al. Clinical predictors of antipsychotic use in children and adolescents with autism spectrum disorders: a historical open cohort study using electronic health records. Eur. Child Adolesc. Psychiatry 25, 649-658 (2016).

29. Steiner, H., Warren, B. L., Van Waes, V. \& Bolaños-Guzmán, C. A. Life-long consequences of juvenile exposure to psychotropic drugs on brain and behavior. Prog. Brain Res. 211, 13-30 (2014). 


\section{Chapter 2.2}

Psychiatric comorbidities and psychotropic medication use in autism: a matched cohort study with ADHD and general population comparator groups in the United Kingdom
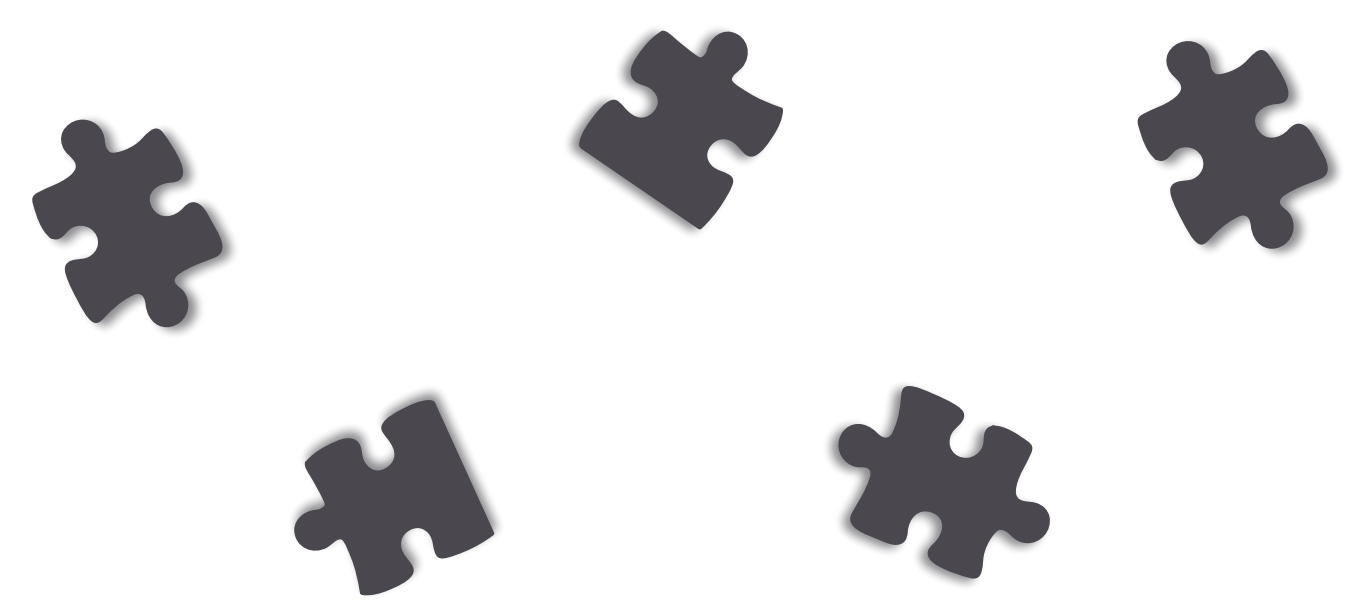


\begin{abstract}
Psychiatric comorbidities and use of psychotropic medications are common among patients with autism spectrum disorder (ASD). However, most previous research used data from the United States (US) and few studies have compared medication use in ASD to control groups, making contextualisation of results difficult. In the United Kingdom (UK), general practitioners play a key role in the management of ASD. We conducted a retrospective, cross-sectional study over calendar year 2015, using primary care data from the UK. We identified a prevalent cohort of ASD cases $(n=10,856)$ and matched control groups of (a) general population ( $n=21,712)$ and (b) attention deficit hyperactivity disorder (ADHD; n=7,058) on age, sex and region. We described psychiatric comorbidities, psychotropic medications, and healthcare utilisation in all three cohorts. Within the ASD cohort, we used multivariable logistic regression models to explore associations between patient characteristics and the outcomes of: any psychotropic medication, polypharmacy, and number of primary care visits. We used conditional logistic regression to compare the ASD and control groups. Psychiatric comorbidities were recorded for $41.5 \%$ of ASD patients; 32.3\% received psychotropic medication and $9.8 \%$ received polypharmacy. Increased age and all psychiatric comorbidities (except conduct disorder) were associated with treatment use. Males were less likely to receive a treatment than females [odds ratio and $95 \%$ confidence interval (OR) $0.74(0.66-0.83)]$. ASD patients were more likely to take psychotropic medications than the general population [OR 4.91 (4.46-5.40)], but less likely compared to ADHD patients [OR 0.40 (0.37-0.44)]. Overall, rates of medication use in the UK were lower than those previously reported in the US.
\end{abstract}

\title{
Lay summary
}

We used electronic medical records from the UK to describe the amount of psychiatric comorbidities, psychotropic medication use and healthcare resource use in ASD. Around one in three people with ASD were prescribed a psychotropic medication, which was more than the general population, but less than for those with ADHD. Increased age, psychiatric comorbidities and female gender were all independently associated with psychotropic medication use. Rates of medication use in the UK were lower than those previously reported in the US. 


\section{Introduction}

Autism spectrum disorder (ASD) is a group of neurobiological disorders of brain development; they are characterised by impairments in social communication and social interaction, as well as the presence of restricted, repetitive interests, behaviours and activities ${ }^{1}$. According to Buescher's report ${ }^{2}$, ASD leads to a huge burden to individual patients and the entire family at both a financial and emotional level and is associated with society burden across health, education and social systems. ASD has been reported across all ethnic, racial and socio-economic groups ${ }^{3}$, with a median reported prevalence rate of approximately $0.62 \%$ worldwide ${ }^{4}$. The increasing prevalence of autism has been reported globally ${ }^{5}$.

There are currently no approved pharmacological treatments for the core symptoms of ASD. Nonetheless previous observational studies have highlighted the high rates of psychotropic medication use in the autistic population ${ }^{6-10}$. These high observed rates are thought to be partly due to the co-occurrence of other mental disorders with ASD ${ }^{11,12}$, but perhaps also due to a lack of available specific pharmacological treatment options. For example, a prior study in the United States (US) showed that one-third of people with ASD aged 3 to 65 years were prescribed a psychotropic medication, even when they did not have other psychiatric conditions ${ }^{13}$.

The majority of studies which have examined psychotropic medication use in ASD have been set in the US. Such studies in the United Kingdom (UK) have generally been limited to smaller sample sizes, less recent data and only to specific drugs classes ${ }^{14-16}$. Moreover, few studies have compared medication use in ASD to control groups; especially not to other neurodevelopmental conditions. The absence of comparisons to such control groups makes contextualisation of results more difficult.

We aimed to conduct a more contemporary study in the UK, with a large number of ASD patients, and compare their pharmacological treatment patterns to two control cohorts -- namely, a general population and a cohort with attention deficit hyperactivity disorder (ADHD). ADHD was deemed an appropriate comparison due to it being another neurodevelopmental condition, affecting a similar age range in onset and also having a higher prevalence in males ${ }^{17}$. We also assessed healthcare resource utilisation in terms of general practitioner (GP) visits and referrals to secondary care. The rationale was to provide a more complete picture of treatment provided for ASD in primary care. 


\section{Methods}

\section{Data source}

This study used data from the Clinical Practice Research Datalink (CPRD) database from the UK. The CPRD data is electronically and routinely captured in the primary care setting by GPs, who are seen as the "gatekeepers" for all non-emergency health matters. Over $98 \%$ of the UK population is registered with a GP ${ }^{18}$, and $7 \%$ of whom are registered at a practice which uses software feeding into the CPRD database. The data includes patient demographics, consultations, diagnoses and prescriptions from primary care, as well as key referrals to, and diagnoses from secondary care. It is representative in terms of age and $\operatorname{sex}^{19}$. A recent study suggested that ASD diagnoses recorded in the CPRD are reliable and can be used with confidence 20 .

\section{Study design and populations}

This was a retrospective, year-long, cohort study set in the calendar year 2015. The study follow-up time was exactly one year for all participants. As such, an inclusion criterion for all patients was continued registration at the same GP practice throughout the whole calendar year. In order to assess baseline characteristics and if prescriptions during 2015 were new or repeated, all subjects were also required to be enrolled during the calendar year 2014. Both the subject and practice level data had to meet the CPRD derived "up to standard" and "patient acceptability" quality criteria throughout 2014 and 2015.

We defined an ASD cohort and two control cohorts of interest. Patients were identified for the ASD cohort if they had at least one diagnosis code for ASD recorded at any time before 1st January 2015. This meant the cohort selected was a prevalent ASD cohort. Given diagnostic uncertainties, individuals were excluded from the ASD cohort if they were below the age of 3 years ${ }^{7}$, or had a record for Rett's syndrome at any time in their file ${ }^{9,21}$.

The control cohorts were: (a) a cohort of people without ASD ("general controls"), and (b) cohort of people with ADHD ("ADHD controls"). All patients without any record of ASD in their patient file were considered as potential general controls. All patients with an ADHD record prior to 1st January 2015, and without record of ASD in their whole patient file were considered as potential ADHD controls. Exact matching was performed randomly and without replacement so that individuals in both control cohorts matched individual ASD cases on year of birth, sex and region of GP practice. Matching each of the comparator cohorts was initially intended with a 2:1 ratio, with the pre-specified option of matching 1:1 if necessitated by available sample size.

\section{Definition of study variables}

The key outcome variables included in the analysis were definitions of comorbid conditions (attention deficit hyperactivity disorder (ADHD), anxiety disorder, bipolar disorder, conduct disorder, depression, epilepsy, intellectual disability, schizophrenia, 
sleep disturbances and obsessive compulsive disorder (OCD)), classes of psychotropic medications (stimulants/atomoxetine, antidepressants, antipsychotics/tranquilizers, hypotensive agents, anticonvulsants and anxiolytics/sedatives/hypnotics), polypharmacy, and resource utilisation.

Each of the comorbidities was considered either absent or present based on whether an associated code was recorded prior to or during the study period (any time prior to 31st December 2015). Each of the psychotropic medications were considered absent or present based on whether there was a prescription recorded within the study period (any time during calendar year 2015). Polypharmacy was defined in accordance with previous research $6,9,13$ as having a days-supply for at least two drugs in different psychotropic medication classes overlapping by 30 consecutive days or more throughout 2015. Additionally, patients with any psychotropic medication prescribed in the study period (calendar year 2015) were split into two categories as follows: (a) "new users" and (b) "continuing users", based on if they had been prescribed at least one psychotropic medication of interest within one year prior to first psychotropic medication in 2015 or not. Finally for medications, the number of days on treatment per psychotropic drug class was calculated based on the quantity prescribed and the recommended numeric daily dose. When the daily dose was missing, we imputed the median daily dose per drug class among non-missing information. When a patient had two prescriptions from the same drug class with overlapping dates, we assumed that treatment supply left from the first prescription was not carried over.

Resource utilisation included the number of visits to primary care, and the number of recorded referrals to secondary care during 2015. The presence of referrals to secondary care is well captured in the database, but the specialty-type of secondary care is generally omitted. Hence, we summarised the most frequent specialties of secondary care, but a more detailed analysis was not possible.

Demographic variables available were age, sex and region. All comorbidities were identified based on Read codes, and drug codes were identified on the bases of the British National Formulary ${ }^{22}$. A full list of codes used is available in the online supplementary material (https://doi.org/10.1002/aur.2040).

\section{Data analysis}

First we described the demographic characteristics of the ASD cohort and both control cohorts by absolute number and percentage, stratifying age into the following groups: 3-4, 5-11, 12-17, 18-24, 25-49 and $\geq 50$ years. We also grouped region to the country level: England, Scotland, Wales and Northern Ireland. In bivariate analyses, we described the percentage of subjects with different comorbidities, psychotropic medications and amount of resource use by age group. 
For the ASD cohort only, the number of days on different psychotropic medications in 2015 (maximum 365 days) was described by means and standard deviations. The prevalence of each medication was also reported within subgroups of subjects where each of the psychiatric comorbidities was observed. The prevalence and days on treatment with each medication, the prevalence of psychiatric comorbidity and frequency of resource use were also reported within the subgroups of new and continuing psychotropic medication users. Also in the ASD cohort only, we fitted separate multivariable logistic regression models for the outcomes "any psychotropic medication", "psychotropic polypharmacy" and "greater than median number of GP visits". We included presence/absence of each psychiatric comorbidity and demographic variables as covariates.

For a comparison of ASD patients with control groups, conditional logistic regression models - taking matching into account - were fitted for the same outcome variables as described above. Age, sex and region were adjusted for by exact matching, and we also adjusted for the presence or absence of any psychiatric comorbidity.

In pre-specified sensitivity analyses, we restricted all regression modelling to the age range 5-24 years. The rationale for this was to see if results remained consistent in a population for whom we might expect fewer unobserved potential sources of bias associated with younger or older age (like uncertainty of diagnosis, other comorbid conditions and factors to do with family home). This is also the same upper age range as used by Murray et al ${ }^{16}$, the prior study most similar to ours. We used R version 3.4.2 for all analyses.

\section{Protocol and ethics approval}

The protocol was submitted to and accepted by the Independent Scientific Advisory Committee (ISAC) of CPRD (reference: 17_103, available in the online supplementary material: https://doi.org/10.1002/aur.2040). The presented data are anonymised. Exact numbers in subgroups with five patients or fewer are not presented, in order to maintain a very low risk of patient identification. In minor deviation from the protocol and the ISAC submission, we added OCD to the investigated psychiatric comorbidities. Failure to account for this in the original protocol was due to oversight.

\section{Results}

\section{Study populations}

Figure 2.2.1 shows the attrition table for selection of the cohorts from the database. A total of 38,753 subjects were identified in CPRD with an ASD record. After applying the selection criteria (see methods), 10,856 subjects remained in the final ASD study cohort. As expected, a higher proportion of participants were male (80.7\%). The mean (SD) age was 18.76 (11.86) years. The majority (62.0\%) resided in England. It was possible to match cases with a 2:1 ratio for general controls $(n=21,712)$ and the ADHD cohort was 
matched 1:1 ( $n=7,058)$. Matching was performed on sex, age, and region, so for these characteristics all matched cohorts were perfectly balanced (see Table 2.2.1). The full ADHD cohort was remarkably similar to the full ASD cohort even before matching (see supplement S.2.2.1).

Figure 2.2.1: Attrition table for patients included in study cohorts

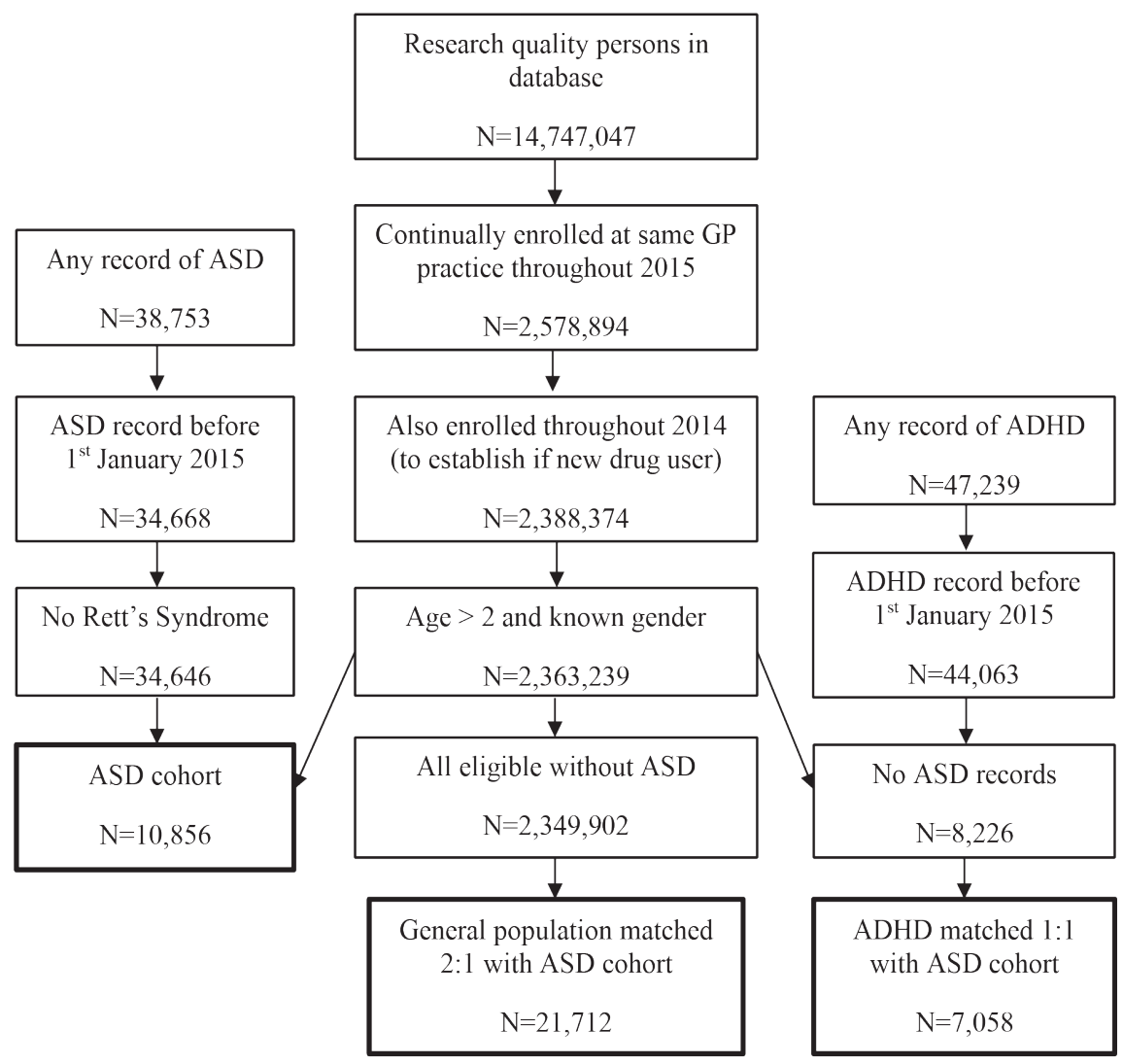

$\mathrm{ADHD}=$ attention deficit hyperactive disorder; $\mathrm{ASD}=$ autism spectrum disorder

\section{Descriptive data}

Forty-two percent of the ASD patients had at least one of the psychiatric comorbidities of interest; with the most frequent being sleep disturbances and ADHD, with 14.9\% and 13.8\% respectively. About one third had at least one of the psychotropic medication classes prescribed (32.3\%). The most frequently prescribed classes were anxiolytics/ sedatives/hypnotics (14.2\%), followed by antidepressants (12.8\%). Polypharmacy was observed in $9.8 \%$ of ASD patients. A sizeable proportion of patients (13.8\%) received medication despite having none of the mental comorbidities. The vast majority of ASD 
patients had at least one GP visit in calendar year 2015 (86.1\%), and seven visits per year on average. About one-fifth of ASD patients had at least one referral to secondary care (18.9\%). The specialties most commonly referred to were: paediatrics; ear, nose and throat; and child and adolescent psychiatry. Information on the specialty referred to was however seldom recorded in the database. Matching to the ADHD cohort did not qualitatively affect the distribution of mental comorbidities and psychotropic drug classes in the ASD cohort.

In the matched ADHD cohort, 28.8\% had any of the selected mental comorbidities. Similarly to the ASD cohort, the most common was sleep disturbances (15.2\%), followed by depression (10.2\%). Almost half the ADHD patients had any psychotropic medication, and by far the most frequently prescribed were stimulants/atomoxetine (34.6\%). Interactions with the health care system were similar to the ASD cohort (GP visits and referrals).

In the matched overall population, mental comorbidities were recorded in 11.9\%, again with sleep disturbances and depression being most common (4.8\% and 4.3\%, respectively). Any psychotropic medication was used by $6.5 \%$.

\section{Detailed patterns in the ASD cohort}

Table 2.2.2 describes the comorbidities and medications in ASD by age group. The presence of any mental comorbidity was increasingly recoded in the higher age groups, starting at 7.3\% in the age group 3-4 years and reaching $76.2 \%$ in those at least 50 years. The same general pattern was observed for all individual comorbidities except ADHD, which peaked at $17.7 \%$ in the age group 12-17 years. The greatest increase in prevalence with age was for depression and anxiety disorder, primarily starting with age group 25-49 years.

The use of any of the psychotropic drug classes steadily increased with age, with $10.9 \%$ in the youngest group to $68.2 \%$ in the oldest group. This pattern was mainly due to increased use of the classes of antidepressants, antipsychotics/tranquilizers and anxiolytics/ sedatives/hypnotics. Polypharmacy increased about 10 percentage points between age groups $18-24$ years and $25-49$ years (from $8.9 \%$ to 19.9\%), and similar then again to the oldest age group (30.6\%).

Patients newly starting a psychotropic medication in 2015 typically started with a single class of treatments (polypharmacy was 6.5\% for new-users vs 34.8\% for continuing users). New users were less often prescribed anticonvulsants, antipsychotic/tranquilizers and stimulants/atomoxetine, while the proportion of patients receiving antidepressants, anxiolytics/sedatives/hypnotics or hypotensives was similar to those having been on any of the classes before. Interactions with the health care system were remarkably similar between the two groups, although new-users had more referrals (31.2\% vs 24.6\%). Full details are presented in Table 2.2.3. 
Table 2.2.1: Baseline demographics, mental comorbidities and psychometric medication use of ASD patients and matched* control groups (ADHD and general population)

\begin{tabular}{|c|c|c|c|c|}
\hline & \multicolumn{2}{|c|}{$\begin{array}{c}\text { ASD vs general population } \\
\text { comparison } \\
\text { (matched } 2: 1 \text { ratio) }\end{array}$} & \multicolumn{2}{|c|}{$\begin{array}{l}\text { ASD vs ADHD comparison } \\
\text { (matched 1:1 ratio) }\end{array}$} \\
\hline & ASD cohort & $\begin{array}{l}\text { General } \\
\text { Population }\end{array}$ & $\begin{array}{c}\text { ASD } \\
\text { (matched to } \\
\text { ADHD) }\end{array}$ & $\begin{array}{c}\text { ADHD } \\
\text { (matched to } \\
\text { ASD) }\end{array}$ \\
\hline$n$ & 10,856 & 21,712 & 7,058 & 7,058 \\
\hline Male & $8,760(80.7)$ & $17,520(80.7)$ & $5,736(81.3)$ & $5,736(81.3)$ \\
\hline \multicolumn{5}{|l|}{ Region } \\
\hline England & $6,730(62.0)$ & $13,460(62.0)$ & $4,412(62.5)$ & $4,412(62.5)$ \\
\hline Northern Ireland & $779(7.2)$ & $1,558(7.2)$ & $400(5.7)$ & $400(5.7)$ \\
\hline Scotland & $1,864(17.2)$ & $3,728(17.2)$ & $1,167(16.5)$ & $1,167(16.5)$ \\
\hline Wales & $1,483(13.7)$ & $2,966(13.7)$ & $1,079(15.3)$ & $1,079(15.3)$ \\
\hline Age (mean (SD))[years] & $18.76(11.86)$ & $18.76(11.86)$ & $20.05(9.91)$ & $20.05(9.91)$ \\
\hline \multicolumn{5}{|l|}{ Age group (years) } \\
\hline $3-4$ & $110(1.0)$ & $220(1.0)$ & 1,082 (15.4) & $1,082(15.4)$ \\
\hline $5-11$ & $2,813(25.9)$ & $5,626(25.9)$ & $* *$ & $* *$ \\
\hline $12-17$ & $3,371(31.1)$ & $6,742(31.1)$ & 2,187 (31.0) & $2,187(31.0)$ \\
\hline $18-24$ & $2,467(22.7)$ & $4,934(22.7)$ & 2,259 (32.0) & $2,259(32.0)$ \\
\hline $25-49$ & $1,667(15.4)$ & $3,334(15.4)$ & $1,368(19.4)$ & $1,368(19.4)$ \\
\hline$\geq 50$ & 428 ( 3.9$)$ & $856(3.9)$ & $162(2.3)$ & $162(2.3)$ \\
\hline \multicolumn{5}{|l|}{ Mental comorbidity } \\
\hline ADHD & $1,495(13.8)$ & $314(1.4)$ & $1,076(15.2)$ & 7,058 (100.0) \\
\hline Anxiety & $826(7.6)$ & $516(2.4)$ & $618(8.8)$ & $430(6.1)$ \\
\hline Bipolar disorder & $83(0.8)$ & $20(0.1)$ & $57(0.8)$ & $33(0.5)$ \\
\hline Conduct disorder & $46(0.4)$ & $16(0.1)$ & $37(0.5)$ & $78(1.1)$ \\
\hline Depression & $919(8.5)$ & $923(4.3)$ & $679(9.6)$ & $722(10.2)$ \\
\hline Epilepsy & $713(6.6)$ & $211(1.0)$ & 518 ( 7.3) & $198(2.8)$ \\
\hline Intellectual disability & 408 ( 3.8$)$ & $15(0.1)$ & $274(3.9)$ & $58(0.8)$ \\
\hline Schizophrenia & $57(0.5)$ & $17(0.1)$ & $39(0.6)$ & $17(0.2)$ \\
\hline Sleep disturbances & $1,620(14.9)$ & $1,035(4.8)$ & $1,099(15.6)$ & $1,075(15.2)$ \\
\hline OCD & $267(2.5)$ & $54(0.2)$ & $202(2.9)$ & $65(0.9)$ \\
\hline Any & $4,505(41.5)$ & $2,593(11.9)$ & $3,167(44.9)$ & $2,032(28.8)$ \\
\hline \multicolumn{5}{|l|}{ Psychotropic medication use } \\
\hline Anticonvulsants & $770(7.1)$ & $251(1.2)$ & $541(7.7)$ & $220(3.1)$ \\
\hline Antidepressants & $1,386(12.8)$ & $872(4.0)$ & $1,039(14.7)$ & $796(11.3)$ \\
\hline Antipsychotics/Tranquilizers & $898(8.3)$ & $91(0.4)$ & $614(8.7)$ & $234(3.3)$ \\
\hline Anxiolytics/Sedatives/Hypnotics & $1,540(14.2)$ & $415(1.9)$ & $941(13.3)$ & $966(13.7)$ \\
\hline Hypotensives & $40(0.4)$ & $11(0.1)$ & $33(0.5)$ & $35(0.5)$ \\
\hline Stimulants/atomoxetine & $713(6.6)$ & $159(0.7)$ & $470(6.7)$ & $2,445(34.6)$ \\
\hline Any & 3,507 (32.3) & $1,415(6.5)$ & $2,397(34.0)$ & $3,354(47.5)$ \\
\hline
\end{tabular}


Table 2.2.1: Baseline demographics, mental comorbidities and psychometric medication use of ASD patients and matched* control groups (ADHD and general population) (cont.)

\begin{tabular}{rrrrr}
\hline & $\begin{array}{r}\text { ASD vs general population } \\
\text { comparison } \\
\text { (matched 2:1 ratio) }\end{array}$ & $\begin{array}{c}\text { ASD vs ADHD comparison } \\
\text { (matched 1:1 ratio) }\end{array}$ \\
\cline { 2 - 5 } & ASD cohort & $\begin{array}{c}\text { General } \\
\text { Population }\end{array}$ & $\begin{array}{c}\text { ASD } \\
\text { (matched to } \\
\text { ADHD) }\end{array}$ & $\begin{array}{c}\text { ADHD } \\
\text { (matched to } \\
\end{array}$ \\
\cline { 2 - 5 } & & & & ASD) \\
\hline Drug classes used concurrently & & & & \\
Polypharmacy & $1,059(9.8)$ & $152(0.7)$ & $709(10.0)$ & $802(11.4)$ \\
Exactly 2 & $803(7.4)$ & $125(0.6)$ & $541(7.7)$ & $696(9.9)$ \\
Exactly 3 & $229(2.1)$ & $25(0.1)$ & $150(2.1)$ & $88(1.2)$ \\
Exactly 4 & $25(0.2)$ & $1(0.0)$ & $16(0.2)$ & $16(0.2)$ \\
Exactly 5 & $2(0.0)$ & $1(0.0)$ & $2(0.0)$ & $2(0.0)$ \\
\hline GP consultation & $9,346(86.1)$ & $15,720(72.4)$ & $5,928(84.0)$ & $6,211(88.0)$ \\
GP consultations (mean (SD)) & $6.93(8.20)$ & $3.69(5.33)$ & $6.68(8.27)$ & $7.05(7.65)$ \\
Referral to secondary care & $2,053(18.9)$ & $2,758(12.7)$ & $1,313(18.6)$ & $1,355(19.2)$ \\
Referrals (mean (SD)) & $0.27(0.67)$ & $0.17(0.51)$ & $0.27(0.69)$ & $0.28(0.69)$ \\
\hline
\end{tabular}

$\mathrm{ADHD}=$ Attention Deficit Hyperactive Disorder, $\mathrm{ASD}=$ Autism Spectrum Disorder, $\mathrm{OCD}=$ Obsessivecompulsive Disorder, $\mathrm{SD}=$ Standard Deviation.

${ }^{*}$ Cohorts are matched on age (exact, not grouped) at index (1st Jan 2015), gender and region (four regions). Data are n(\%) for year 2015 unless stated otherwise. ${ }^{* *}$ Adjacent cells combined as patient count in 3-4 age group is $<5$. 


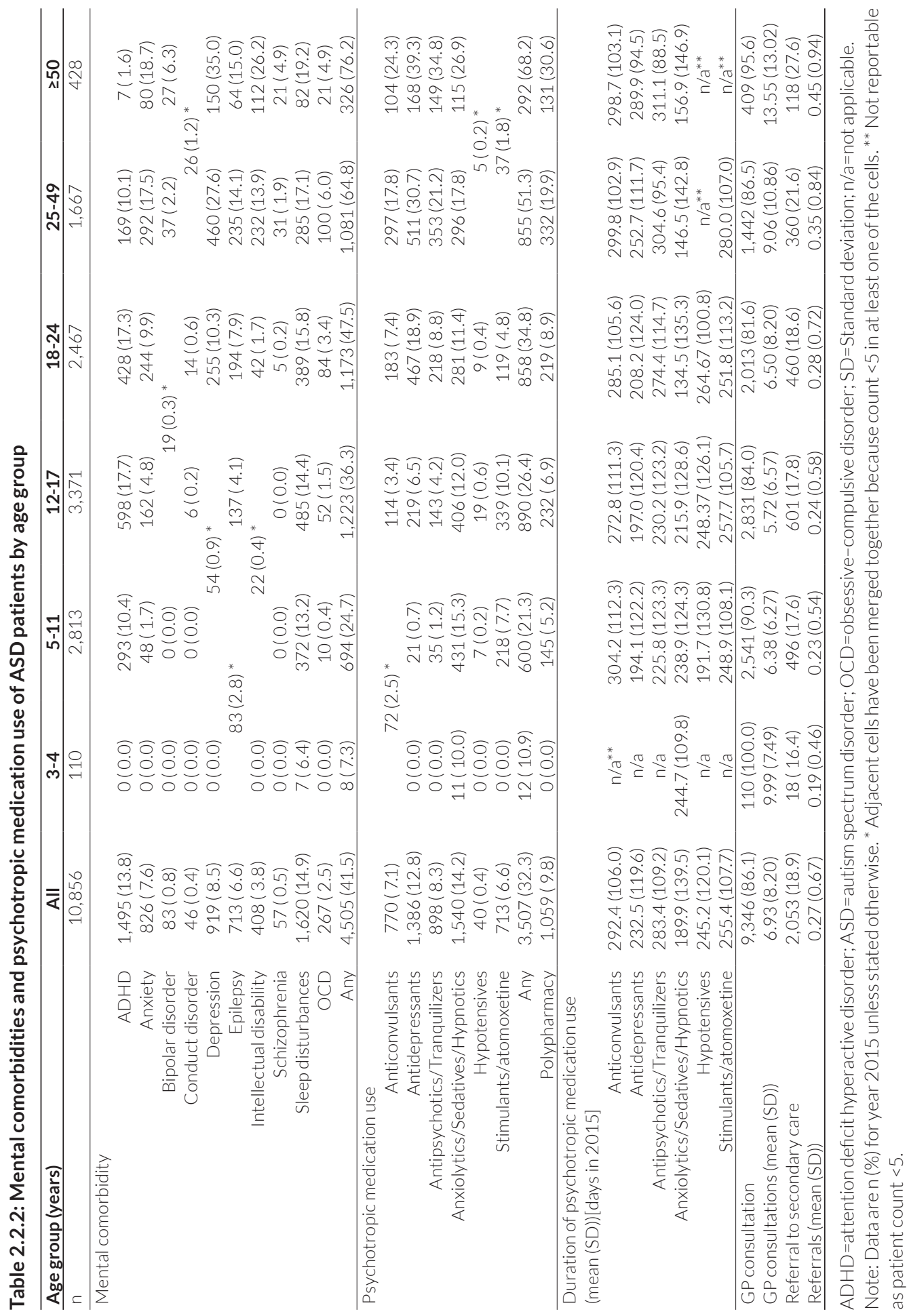


Table 2.2.3: Mental comorbidities and psychometric medication use of ASD patients by treatment status

\begin{tabular}{|c|c|c|}
\hline & $\begin{array}{l}\text { New psychotropic } \\
\text { medication user }\end{array}$ & $\begin{array}{c}\text { Continuing psychotropic } \\
\text { medication user }\end{array}$ \\
\hline$n$ & 567 & 2,940 \\
\hline \multicolumn{3}{|l|}{ Mental comorbidity } \\
\hline ADHD & $111(19.6)$ & 729 ( 24.8) \\
\hline Anxiety & $72(12.7)$ & $445(15.1)$ \\
\hline Bipolar disorder & $<5(<0.9)^{*}$ & $74(2.5)$ \\
\hline Conduct disorder & $<5(<0.9)^{*}$ & $24(0.8)$ \\
\hline Depression & $90(15.9)$ & $584(19.9)$ \\
\hline Epilepsy & $27(4.8)$ & $561(19.1)$ \\
\hline Intellectual disability & $12(2.1)$ & $283(9.6)$ \\
\hline Schizophrenia & $<5(<0.9)^{*}$ & $38(1.3)$ \\
\hline Sleep disturbances & $145(25.6)$ & $733(24.9)$ \\
\hline OCD & $15(2.6)$ & $168(5.7)$ \\
\hline Any & $343(60.5)$ & $2,290(77.9)$ \\
\hline \multicolumn{3}{|l|}{ Psychotropic medication use } \\
\hline Anticonvulsants & $29(5.1)$ & $741(25.2)$ \\
\hline Antidepressants & $232(40.9)$ & $1,154(39.3)$ \\
\hline Antipsychotics/Tranquilizers & $53(9.3)$ & $845(28.7)$ \\
\hline Anxiolytics/Sedatives/Hypnotics & $275(48.5)$ & $1,265(43.0)$ \\
\hline Hypotensives & $<5(<0.9)^{*}$ & $36(1.2)$ \\
\hline Stimulants/atomoxetine & $80(14.1)$ & $633(21.5)$ \\
\hline Any & $567(100.0)$ & $2,940(100.0)$ \\
\hline Polypharmacy & $37(6.5)$ & $1,022(34.8)$ \\
\hline \multicolumn{3}{|l|}{$\begin{array}{l}\text { Duration of psychotropic medication } \\
\text { use (mean (SD)) [days in 2015]** }\end{array}$} \\
\hline Anticonvulsants & $240.5(115.8)$ & $301.3(99.0)$ \\
\hline Antidepressants & $219.4(119.3)$ & $262.7(107.9)$ \\
\hline Antipsychotics/Tranquilizers & $192.8(125.9)$ & $298.1(97.2)$ \\
\hline Anxiolytics/Sedatives/Hypnotics & $162.1(133.3)$ & $218.8(135.9)$ \\
\hline Hypotensives & $\mathrm{n} / \mathrm{a}^{*}$ & $260.1(114.5)$ \\
\hline Stimulants/atomoxetine & $217.3(120.5)$ & $275.9(94.8)$ \\
\hline GP consultations & $566(99.8)$ & $2885(98.1)$ \\
\hline GP consultations (mean (SD)) & $11.87(9.70)$ & $12.01(10.59)$ \\
\hline Referrals to secondary care & $177(31.2)$ & $723(24.6)$ \\
\hline Referrals (mean (SD)) & $0.48(0.87)$ & $0.38(0.84)$ \\
\hline
\end{tabular}

$\mathrm{ADHD}=$ attention deficit hyperactive disorder; $\mathrm{ASD}=$ autism spectrum disorder; $\mathrm{OCD}=$ obsessivecompulsive disorder; SD=standard deviation; $n / a$, not applicable. *Not reportable as patient count $<5$.

${ }^{* *}$ Adjusted to whole-year for new drug users, to allow comparison with continuing users.

Data are n(\%) for year 2015 unless stated otherwise.

\section{Regression analyses in ASD cohort}

The multivariable logistic regression demonstrated - similarly to the descriptive analysis - that increasing age was associated with both any psychotropic medication use and polypharmacy in patients with ASD (Table 2.2.4). Male patients had lower odds of receiving any class of psychotropic medication [odds ratio and 95\% confidence interval (OR) 0.74 (0.660.83)] or two or more psychotropic medications at the same time [OR 0.80 (0.68-0.95)]. 
Table 2.2.4: Association of psychotropic medication use and visits to GP with patient characteristics and comorbidities in ASD patients (multivariable logistic regression, GP visits dichotomised at median)

\begin{tabular}{|c|c|c|c|c|c|c|}
\hline & \multicolumn{2}{|c|}{$\begin{array}{c}\text { No medication } \\
\text { vs any medication }\end{array}$} & \multicolumn{2}{|c|}{$\begin{array}{l}\text { No polypharmacy } \\
\text { vs polypharmacy }\end{array}$} & \multicolumn{2}{|c|}{$\begin{array}{c}<5 \text { GP visits } \\
\text { vs } \geq 5 \text { GP visits }\end{array}$} \\
\hline & $\begin{array}{c}\text { Odds ratio }(95 \% \\
\mathrm{Cl})\end{array}$ & $p$-value & $\begin{array}{l}\text { Odds ratio } \\
(95 \% \mathrm{Cl})\end{array}$ & $\begin{array}{l}\text { Odds ratio } \\
(95 \% \mathrm{Cl})\end{array}$ & $p$-value & $\begin{array}{c}\text { Odds ratio } \\
(95 \% \mathrm{Cl})\end{array}$ \\
\hline \multicolumn{7}{|l|}{ Age group (years) } \\
\hline $3-4$ & Ref. & & $n / a^{*}$ & & Ref. & \\
\hline $5-11$ & $\begin{array}{c}1.65 \\
(0.92,3.25)\end{array}$ & 0.119 & Ref. & & $\begin{array}{c}0.25 \\
(0.16,0.39)\end{array}$ & $<0.01$ \\
\hline $12-17$ & $\begin{array}{c}1.73 \\
(0.96,3.41)\end{array}$ & 0.087 & $\begin{array}{c}1.07 \\
(0.85,1.33)\end{array}$ & 0.574 & $\begin{array}{c}0.16 \\
(0.10,0.25)\end{array}$ & $<0.01$ \\
\hline $18-24$ & $\begin{array}{c}1.93 \\
(1.07,3.80)\end{array}$ & 0.041 & $\begin{array}{c}1.08 \\
(0.86,1.37)\end{array}$ & 0.513 & $\begin{array}{c}0.14 \\
(0.09,0.22)\end{array}$ & $<0.01$ \\
\hline $25-49$ & $\begin{array}{c}2.54 \\
(1.40,5.04)\end{array}$ & $<0.01$ & $\begin{array}{c}2.03 \\
(1.59,2.61)\end{array}$ & $<0.01$ & $\begin{array}{c}0.16 \\
(0.10,0.26)\end{array}$ & $<0.01$ \\
\hline$\geq 50$ & $\begin{array}{c}5.14 \\
(2.73,10.51)\end{array}$ & $<0.01$ & $\begin{array}{c}3.50 \\
(2.52,4.83)\end{array}$ & $<0.01$ & $\begin{array}{c}0.37 \\
(0.22,0.61)\end{array}$ & $<0.01$ \\
\hline \multicolumn{7}{|l|}{ Gender } \\
\hline Female & Ref. & & Ref. & & Ref. & \\
\hline Male & $\begin{array}{c}0.74 \\
(0.66,0.83)\end{array}$ & $<0.01$ & $\begin{array}{c}0.80 \\
(0.68,0.95) \\
\end{array}$ & 0.011 & $\begin{array}{c}0.54 \\
(0.49,0.60)\end{array}$ & $<0.01$ \\
\hline \multicolumn{7}{|l|}{ Geographic region } \\
\hline England & Ref. & & Ref. & & Ref. & \\
\hline Northern Ireland & $\begin{array}{c}1.29 \\
(1.07,1.54)\end{array}$ & $<0.01$ & $\begin{array}{c}1.41 \\
(1.06,1.85)\end{array}$ & 0.015 & $\begin{array}{c}1.16 \\
(1.00,1.36)\end{array}$ & 0.057 \\
\hline Scotland & $\begin{array}{c}1.49 \\
(1.32,1.70)\end{array}$ & $<0.01$ & $\begin{array}{c}1.40 \\
(1.16,1.69)\end{array}$ & $<0.01$ & $\begin{array}{c}0.81 \\
(0.73,0.91)\end{array}$ & $<0.01$ \\
\hline Wales & $\begin{array}{c}1.18 \\
(1.02,1.35) \\
\end{array}$ & 0.025 & $\begin{array}{c}0.95 \\
(0.76,1.18)\end{array}$ & 0.632 & $\begin{array}{c}1.01 \\
(0.90,1.14)\end{array}$ & 0.882 \\
\hline \multicolumn{7}{|l|}{ Comorbid conditions } \\
\hline ADHD & $\begin{array}{c}4.67 \\
(4.12,5.30)\end{array}$ & $<0.01$ & $\begin{array}{c}4.69 \\
(3.97,5.54)\end{array}$ & $<0.01$ & $\begin{array}{c}2.16 \\
(1.92,2.43)\end{array}$ & $<0.01$ \\
\hline Anxiety & $\begin{array}{c}2.39 \\
(2.00,2.85)\end{array}$ & $<0.01$ & $\begin{array}{c}1.48 \\
(1.19,1.84)\end{array}$ & $<0.01$ & $\begin{array}{c}2.16 \\
(1.83,2.57)\end{array}$ & $<0.01$ \\
\hline Bipolar disorder & $\begin{array}{c}8.68 \\
(4.13,20.64)\end{array}$ & $<0.01$ & $\begin{array}{c}5.51 \\
(3.33,9.16)\end{array}$ & $<0.01$ & $\begin{array}{c}2.53 \\
(1.40,4.87)\end{array}$ & $<0.01$ \\
\hline Conduct disorder & $\begin{array}{c}1.01 \\
(0.47,2.14)\end{array}$ & 0.991 & $\begin{array}{c}1.36 \\
(0.61,2.88)\end{array}$ & 0.435 & $\begin{array}{c}0.76 \\
(0.39,1.47)\end{array}$ & 0.404 \\
\hline Depression & $\begin{array}{c}4.82 \\
(4.02,5.78)\end{array}$ & $<0.01$ & $\begin{array}{c}2.11 \\
(1.70,2.61)\end{array}$ & $<0.01$ & $\begin{array}{c}2.25 \\
(1.89,2.67)\end{array}$ & $<0.01$ \\
\hline Epilepsy & $\begin{array}{c}12.76 \\
(10.36,15.81)\end{array}$ & $<0.01$ & $\begin{array}{c}5.09 \\
(4.18,6.19)\end{array}$ & $<0.01$ & $\begin{array}{c}3.49 \\
(2.90,4.22)\end{array}$ & $<0.01$ \\
\hline Intellectual disability & $\begin{array}{c}3.29 \\
(2.52,4.31)\end{array}$ & $<0.01$ & $\begin{array}{c}3.33 \\
(2.56,4.33)\end{array}$ & $<0.01$ & $\begin{array}{c}2.28 \\
(1.77,2.95)\end{array}$ & $<0.01$ \\
\hline Schizophrenia & $\begin{array}{c}2.31 \\
(1.19,4.65)\end{array}$ & 0.016 & $\begin{array}{c}1.60 \\
(0.83,2.98)\end{array}$ & 0.150 & $\begin{array}{c}2.79 \\
(1.39,6.09)\end{array}$ & $<0.01$ \\
\hline Sleep disturbances & $\begin{array}{c}2.65 \\
(2.34,3.00)\end{array}$ & $<0.01$ & $\begin{array}{c}1.91 \\
(1.61,2.24)\end{array}$ & $<0.01$ & $\begin{array}{c}1.82 \\
(1.62,2.04)\end{array}$ & $<0.01$ \\
\hline OCD & $\begin{array}{c}3.29 \\
(2.43,4.46)\end{array}$ & $<0.01$ & $\begin{array}{c}2.92 \\
(2.13,3.97)\end{array}$ & $<0.01$ & $\begin{array}{c}1.67 \\
(1.27,2.22)\end{array}$ & $<0.01$ \\
\hline
\end{tabular}

*n/a: not applicable as n = 0. Model diagnostics for "any drug": McFaddon R-squared=0.22; Nagelkerke R-squared=0.34; area under curve $(A \cup C)=0.81$. Model diagnostics for "polypharmacy": McFaddon R-squared=0.19; Nagelkerke R-squared=0.24; $A \cup C=0.80$. Model diagnostics for visits to GP: McFaddon R-squared $=0.08$; Nagelkerke R-squared $=0.15 ; \mathrm{AUC}=0.76 . \mathrm{ADHD}=$ attention deficit hyperactive disorder; $A S D=$ autism spectrum disorder; $O C D=$ obsessive-compulsive disorder. Ref=Reference group 
All types of mental comorbidities except conduct disorder increased the odds of receiving any psychotropic medication. Subjects with epilepsy [OR 12.76 (10.36-15.81)] and bipolar disorder [OR 8.68 (4.13-20.64)] were the most likely to receive medication. Epilepsy [OR 5.09 (4.18-6.19)] and bipolar disorder [OR 5.51 (3.33-9.16)] were also the comorbidities most strongly associated with polypharmacy. Similarly to any psychotropic medication, there was no association between conduct disorder and polypharmacy. Again, all other types of mental comorbidities increased the odds of polypharmacy, although schizophrenia was only directionally related and not statistically significant.

Apart from conduct disorder, all comorbidities were significantly associated with the intensity of GP consultations. Male ASD patients were also much more likely to have a low intensity of GP interactions [OR 0.54 (0.49-0.60)]. The 3-4 years age group had by far the highest frequency of GP consultations, followed by those over 50 and then those aged 5-11 years. The proportion of ASD patients with high GP visits frequency was rather similar in all age groups between 12 and 49 years.

ASD patients in England were least likely to receive psychotropic medications, and ASD patients in Scotland were least likely to have a high frequency of GP visits. In sensitivity analyses, the above findings were very similar when repeated in the analysis population with age range 5 -24 years.

\section{Comparisons of ASD cohort to control cohorts}

Compared to an age-, gender-, and region-matched cohort from the general population, and adjusting for comorbidities, ASD was associated with a substantially increased likelihood of receiving any psychotropic medication [OR 4.91 (4.46-5.40)], or polypharmacy [OR 9.60 (7.72-11.93)]. ASD patients also had twice the odds of a high intensity of GP visits [OR 2.08 (1.97-2.21)].

In comparison to the matched cohort with ADHD and adjusting for comorbidities however, the odds were about halved for all three outcomes (any psychotropic medication use: OR 0.40 (0.37-0.44); polypharmacy: OR 0.60 (0.52-0.68); and GP visit intensity: OR 0.61 (0.57-0.66)). Mainly this was attributable to the higher frequency of stimulant/ atomoxetine prescriptions (6.7\% in ASD vs 34.6\% in ADHD).

Table 2.2.5 demonstrates that in crude analyses (without adjusting for comorbidities), these associations were directionally the same and also statistically significant. Once more, in sensitivity analyses, these results remained very similar when restricting the age of all cohorts to range from 5-24 years. 
Table 2.2.5: Conditional logistic regressions in matched groups: control groups versus ASD patients

\begin{tabular}{|c|c|c|c|c|c|}
\hline \multirow[t]{2}{*}{ Comparison } & \multirow[t]{2}{*}{ Outcome } & \multicolumn{2}{|c|}{ Crude model* } & \multicolumn{2}{|c|}{ Adjusted model ${ }^{* *}$} \\
\hline & & $\begin{array}{l}\text { Odds ratio } \\
(95 \% \mathrm{Cl})\end{array}$ & $p$-value & $\begin{array}{l}\text { Odds ratio } \\
\text { (95\% Cl) }\end{array}$ & $p$-value \\
\hline \multicolumn{6}{|l|}{$\begin{array}{r}\text { General population } \\
\text { vs ASD }\end{array}$} \\
\hline & Any psychotropic medication & $\begin{array}{c}8.20 \\
(7.57,8.88)\end{array}$ & $<0.01$ & $\begin{array}{c}4.91 \\
(4.46,5.40)\end{array}$ & $<0.01$ \\
\hline & Polypharmacy & $\begin{array}{c}16.51 \\
(13.72,19.86)\end{array}$ & $<0.01$ & $\begin{array}{c}9.60 \\
(7.72,11.93)\end{array}$ & $<0.01$ \\
\hline & 5 or more primary care visits & $\begin{array}{c}2.87 \\
(2.72,3.02)\end{array}$ & $<0.01$ & $\begin{array}{c}2.08 \\
(1.97,2.21)\end{array}$ & $<0.01$ \\
\hline \multicolumn{6}{|l|}{ ADHD vs ASD } \\
\hline & Any psychotropic medication & $\begin{array}{c}0.58 \\
(0.54,0.62)\end{array}$ & $<0.01$ & $\begin{array}{c}0.40 \\
(0.37,0.44)\end{array}$ & $<0.01$ \\
\hline & Polypharmacy & $\begin{array}{c}0.87 \\
(0.78,0.97)\end{array}$ & 0.011 & $\begin{array}{c}0.60 \\
(0.52,0.68)\end{array}$ & $<0.01$ \\
\hline & 5 or more primary care visits & $\begin{array}{c}0.75 \\
(0.70,0.81)\end{array}$ & $<0.01$ & $\begin{array}{c}0.61 \\
(0.57,0.66)\end{array}$ & $<0.01$ \\
\hline
\end{tabular}

Each cell represents a different model. Odds ratios are for ASD compared to control groups as reference.

* Crude comparison of cohorts which are already matched for age, gender and region

**Also adjusted for presence of any psychiatric comorbidity (yes/no).

$\mathrm{ADHD}=$ attention deficit hyperactive disorder; $\mathrm{ASD}=$ autism spectrum disorder.

\section{Discussion}

This study shows that ASD patients in the UK have a high psychiatric comorbidity burden and substantial use of psychotropic medications. Findings are largely consistent with the most comparable previous study set in primary care in the UK, in which Murray et al ${ }^{16}$ explored pharmacological treatments in ASD using The Health Improvement Network (THIN) database, between 1992 and 2008. Murray's sample was about half the size of the sample in this present study and only included people with ASD up until age 24 years. Thirty-seven percent of ASD participants in Murray's study had a psychiatric comorbidity compared to $41.5 \%$ in the present study, and rates of any psychotropic medication use were $29.0 \%$ and $32.3 \%$ respectively. Overall this provides reassurance that the data used reflects practice across the UK and that findings can be reproduced. Our study extended the work by Murray et al, by providing more recent data, expanding the age range studied and most notably, making comparisons to control cohorts.

A major finding of ours was that females with ASD were far more likely to receive psychotropic medications than males, even when controlling for specific comorbidities. This could be additional support to the growing evidence that ASD is underdiagnosed 
in females ${ }^{23-25}$. In a recent meta-analysis, Loomes et al ${ }^{26}$ concluded that the true male to female ratio in ASD is closer to 3:1, rather than 4:1, which we observed in this study. It follows, if diagnosed females generally have more severe symptoms, reasonably we would expect those females to be in need of more care and more treatment. White et al ${ }^{27}$, for example, reported that females with ASD face more difficulties with activities of daily living than their male counterparts. Also consistent with this finding was that female gender in our study was associated with more GP visits. Murray et al ${ }^{16}$ also observed higher psychotropic treatment rates in females with ASD in the UK. A multitude of other gender related, but unmeasured symptoms, such as functional social behaviour, aggression or self-injurious tendencies might also play a role in the choice of whether to initiate psychotropic treatment or not ${ }^{28-30}$.

We also extended previous work by showing that psychotropic medications are far more likely to be given to people with ASD than the general population, even when controlling for psychiatric comorbidities. This suggests that prescriptions of psychotropic medications are sometimes actually targeted at ASD itself; highlighting an unmet medical need in ASD targeted medication. Indeed, 14\% of participants in this study were prescribed psychotropic medication despite having no records of corresponding psychiatric comorbidities. An alternative explanation for some, but likely not all of this discrepancy, is that some diagnoses recorded in secondary and specialist care may not have been manually entered into the CPRD by the GP. The rate of psychotropic treatment in ADHD was higher than in ASD, but this was driven by the use of stimulants/atomoxetine, which are approved in ADHD, while no medication is currently approved in the UK to treat ASD.

Another novel finding was that anti-depressants and anxiolytics/sedatives/hypnotics are the most common psychotropic medications initially prescribed to people with ASD. Other psychotropic medication classes are typically added later. A general point however, is that the reason for prescription is not recorded in CPRD, nor are medications bought over-the-counter.

\section{Comparison to non-UK setting}

Previous findings in the US are systematically different in that the rate of psychotropic medication use is much higher, about double as high in fact. In 2014, psychotropic medication use among ASD patients was $64 \%$ in a database of privately insured individuals and $69 \%$ covered by Medicaid ${ }^{13}$. Other studies set in the US had largely similar numbers, for example Mandell et al with $56 \%^{6}$, Schubart et al with $65 \%^{8}$, and Spencer et al with $64 \%$

9. Contrasts from the latter three studies to the UK are even more significant given they included only children and adolescents, yet a consistent finding throughout has been that greater age is among the strongest predictors for treatment.

Sizeable ( $n>1000$ ) European studies have been conducted by Dalsgaard et al ${ }^{31}$ and Bachmann et al ${ }^{32}$ in Finland $(n=1,577)$ and Germany $(n=1,124)$, respectively. Bachmann 
et al reported an annual psychotropic prevalence rate of 33\% in Germany, while Dalsgaard et al only studied medications for ADHD, but recorded an annual prevalence rate of $16 \%$. Like this study, Dalsgaard et al also unsurprisingly confirmed, that such medications (methylphenidate, dexamphetamine and atomoxetine) were more commonly prescribed in ADHD verses ASD. Both studies again limited analyses to children and adolescents. Nevertheless, these results are more in-line with our findings, confirming that psychotropic medication in ASD is more limited in Europe than in the US. Both studies were also included in a systematic review by Jobski et al ${ }^{33}$, who reported a crude median overall psychotropic medication rate of 45.7\%: higher than our estimate for the UK. The review included participants from Europe, the Middle East, and South Africa, but overwhelmingly from North America (37/47 studies and approximately $94 \%$ of participants were from the US or Canada). This demonstrates the need for studies like the present one to investigate ASD populations outside the US.

The proportion of ASD patients with a record of another mental condition is also markedly higher in the US than in the UK. Houghton et al ${ }^{13}$ estimated that $63 \%$ with commercial insurance in the US and $71 \%$ in Medicaid had one or more mental comorbidities compared with only $42 \%$ in our current study. Lower recorded rates of comorbidity in the UK could partly be due to the fact that CPRD is an electronic medical record (EMR) database, where medical history is stored and is retrievable by GPs after only entering the information once. This is in contrast to insurance claims data, where diagnosis codes are supplied at each consultation for reimbursement purposes, thus potentially inflating the rate of comorbidities. We took measures to account for this where possible, by using all prior diagnoses in CPRD, rather than only using the diagnoses appearing during the study period like typically done in previous ASD claims data analyses 6,10,13.

Finally, results from this study also suggest that GPs in the UK play an important role in the overall care of people with ASD, because the ratio of visits to primary care verses secondary care is very high. Almost all participants in our study had a consultation with the GP during the one year study period. This is in line with the findings of Unigwe et al ${ }^{34}$, whose survey of GPS reported that they play a "key role" in the management of ASD, despite approximately 40\% "never having received formal training" and being "divided about the remit of the GP" in ASD-care. This could call for more guidance about ASD to be given to GPs in the UK.

\section{Limitations and possible extensions}

There are several limitations to the present study. Firstly, there are no standardised definitions of diseases in CPRD (i.e. Read code lists for diseases, including ASD). Thus, inconsistent definitions may hamper comparability between different studies. Wherever possible we used existing code lists from other published CPRD studies for ASD and the other mental comorbidities of interest. Secondly, as mentioned, not all diagnoses made in secondary care are guaranteed to be reported to the GP and entered in the CPRD. 
Warranting further research is the need to quantify the types and frequencies of nondrug therapies available for and accessed by those with ASD. This would provide an even more well-rounded understanding of ASD care and allow further comparisons between different countries. For example, Salomone et al ${ }^{35}$ identified a lower rate of early childhood interventions in the UK compared to other European countries (except Ireland).

\section{Conclusion}

This study demonstrates prescription of psychotropic medications to people with ASD and a high mental comorbidity burden. GPs play an important role in ASD care in the UK, as evidenced by the high consultation rate observed. The rate of psychotropic medication use in the UK however, is distinctly lower than in the US. 


\section{References}

1. Maenner, M. J. et al. Potential impact of DSM-5 criteria on autism spectrum disorder prevalence estimates. JAMA Psychiatry 71, 292-300 (2014).

2. Buescher, A. V. S., Cidav, Z., Knapp, M. \& Mandell, D. S. Costs of autism spectrum disorders in the United Kingdom and the United States. JAMA Pediatr. 168, 721-728 (2014).

3. Ivanov, H. Y., Stoyanova, V. K., Popov, N. T. \& Vachev, T. I. Autism Spectrum Disorder - A Complex Genetic Disorder. Folia Med. (Plovdiv) 57, 19-28 (2015).

4. Elsabbagh, M. et al. Global prevalence of autism and other pervasive developmental disorders. Autism Res. Off. J. Int. Soc. Autism Res. 5, 160-179 (2012).

5. Manning-Courtney, P. et al. Autism spectrum disorders. Curr. Probl. Pediatr. Adolesc. Health Care 43, 2-11 (2013).

6. Mandell, D. S. et al. Psychotropic medication use among Medicaid-enrolled children with autism spectrum disorders. Pediatrics 121, e441-448 (2008).

7. Oswald, D. P. \& Sonenklar, N. A. Medication use among children with autism spectrum disorders. J. Child Adolesc. Psychopharmacol. 17, 348-355 (2007).

8. Schubart, J. R., Camacho, F. \& Leslie, D. Psychotropic medication trends among children and adolescents with autism spectrum disorder in the Medicaid program. Autism Int. J. Res. Pract. 18, 631-637 (2014).

9. Spencer, D. et al. Psychotropic medication use and polypharmacy in children with autism spectrum disorders. Pediatrics 132, 833-840 (2013).

10. Vohra, R. et al. Prescription Drug Use and Polypharmacy Among Medicaid-Enrolled Adults with Autism: A Retrospective Cross-Sectional Analysis. Drugs - Real World Outcomes 3, 409-425 (2016).
11. Accordino, R. E., Kidd, C., Politte, L. C., Henry, C. A. \& McDougle, C. J. Psychopharmacological interventions in autism spectrum disorder. Expert Opin. Pharmacother. 17, 937-952 (2016).

12. Cummings, J. R. et al. Health Services Utilization among Children with and without Autism Spectrum Disorders. J. Autism Dev. Disord. 46, 910-920 (2016).

13. Houghton, R., Ong, R. C. \& Bolognani, F. Psychiatric comorbidities and use of psychotropic medications in people with autism spectrum disorder in the United States. Autism Res. Off. J. Int. Soc. Autism Res. 10, 2037-2047 (2017).

14. Downs, J. et al. Clinical predictors of antipsychotic use in children and adolescents with autism spectrum disorders: a historical open cohort study using electronic health records. Eur. Child Adolesc. Psychiatry 25, 649-658 (2016).

15. Morgan, S. \& Taylor, E. Antipsychotic drugs in children with autism. BMJ 334, 1069-1070 (2007)

16. Murray, M. L. etal. Pharmacological treatments prescribed to people with autism spectrum disorder (ASD) in primary health care. Psychopharmacology (Berl.) 231, 1011-1021 (2014).

17. Leitner, Y. The Co-Occurrence of Autism and Attention Deficit Hyperactivity Disorder in Children - What Do We Know? Front. Hum. Neurosci. 8, 268 (2014).

18. NHS Digital. Attribution Data Set GPRegistered Populations Scaled to ONS Population Estimates - 2011 - NHS Digital. https://digital.nhs.uk/catalogue/ PUB05054; Accessed 12 Nov 2017.

19. Herrett, E. et al. Data Resource Profile: Clinical Practice Research Datalink (CPRD). Int. J. Epidemiol. 44, 827-836 (2015). 
20. Hagberg, K. W. \& Jick, S. S. Validation of autism spectrum disorder diagnoses recorded in the Clinical Practice Research Datalink, 1990-2014. Clin. Epidemiol. 9, 475-482 (2017).

21. Burke, J. P. et al. Does a claims diagnosis of autism mean a true case? Autism Int. J. Res. Pract. 18, 321-330 (2014).

22. British National Formulary 67th Revised edition. (Pharmaceutical Press, 2014).

23. Begeer, S. et al. Sex Differences in the Timing of Identification Among Children and Adults with Autism Spectrum Disorders. J. Autism Dev. Disord. 43, 1151-1156 (2013).

24. Gould, J. \& Ashton-Smith, J. Missed diagnosis or misdiagnosis? Girls and women on the autism spectrum. Good Autism Pract. GAP 12, 34-41 (2011).

25. Wilson, C. E. et al. Does sex influence the diagnostic evaluation of autism spectrum disorder in adults? Autism 20, 808-819 (2016).

26. Loomes, R., Hull, L. \& Mandy, W. P. L. What Is the Male-to-Female Ratio in Autism Spectrum Disorder? A Systematic Review and Meta-Analysis. J. Am. Acad. Child Adolesc. Psychiatry 56, 466-474 (2017).

27. White, E. I. et al. Sex differences in parent-reported executive functioning and adaptive behavior in children and young adults with autism spectrum disorder. Autism Res. 10, 1653-1662 (2017).

28. Bresin, K. \& Schoenleber, M. Gender differences in the prevalence of nonsuicidal self-injury: A meta-analysis. Clin. Psychol. Rev. 38, 55-64 (2015).

29. Halladay, A. K. et al. Sex and gender differences in autism spectrum disorder: summarizing evidence gaps and identifying emerging areas of priority. Mol. Autism 6, 36 (2015).

30. Summers, J. et al. Self-Injury in Autism Spectrum Disorder and Intellectual Disability: Exploring the Role of Reactivity to Pain and Sensory Input. Brain Sci. 7, 140 (2017).
31. Dalsgaard, S., Nielsen, H. S. \& Simonsen, M. Five-fold increase in national prevalence rates of attention-deficit/ hyperactivity disorder medications for children and adolescents with autism spectrum disorder, attentiondeficit/hyperactivity disorder, and other psychiatric disorders: a Danish register-based study. J. Child Adolesc. Psychopharmacol. 23, 432-439 (2013).

32. Bachmann, C. J., Manthey, T., KampBecker, I., Glaeske, G. \& Hoffmann, F. Psychopharmacological treatment in children and adolescents with autism spectrum disorders in Germany. Res. Dev. Disabil. 34, 2551-2563 (2013).

33. Jobski, K., Höfer, J., Hoffmann, F. \& Bachmann, C. Use of psychotropic drugs in patients with autism spectrum disorders: a systematic review. Acta Psychiatr. Scand. 135, 8-28 (2017).

34. Unigwe, S. et al. GPs' confidence in caring for their patients on the autism spectrum: an online self-report study. Br. J. Gen. Pract. J. R. Coll. Gen. Pract. 67, e445-e452 (2017).

35. Salomone, E. et al. Use of early intervention for young children with autism spectrum disorder across Europe. Autism Int. J. Res. Pract. 20, 233-249 (2016). 


\section{S.2.2.1: Baseline demographics of full ADHD population}

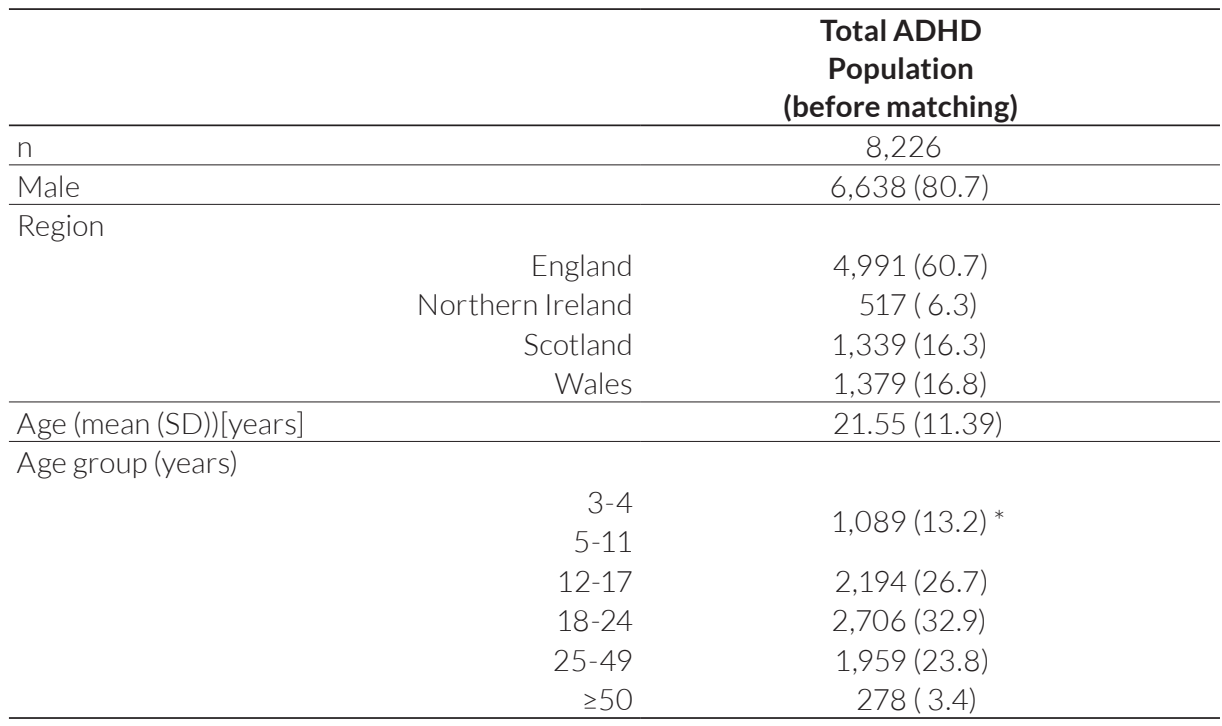

Abbreviations: ADHD, attention deficit hyperactive disorder; SD, standard deviation;

* Adjacent cells have been merged together because count $<5$ in at least one of the cells.

Data are n(\%) unless stated otherwise. 

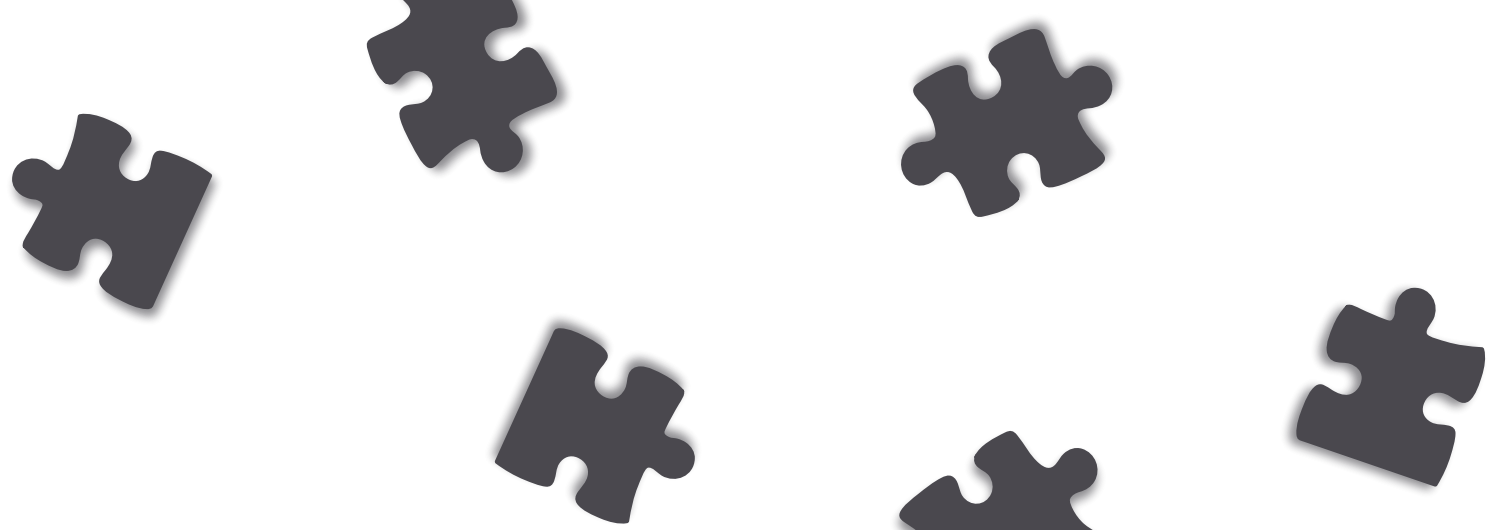

Note: the published manuscript comes with a large online appendix (42 pages). A selection of supplementary tables and figures has been added to the current chapter. The full appendix is available on the publishers' website in the supporting information section: https://doi.org/10.1002/aur.2070 


\section{Chapter 2.3}

\section{Treatment patterns in children with autism in the United States}
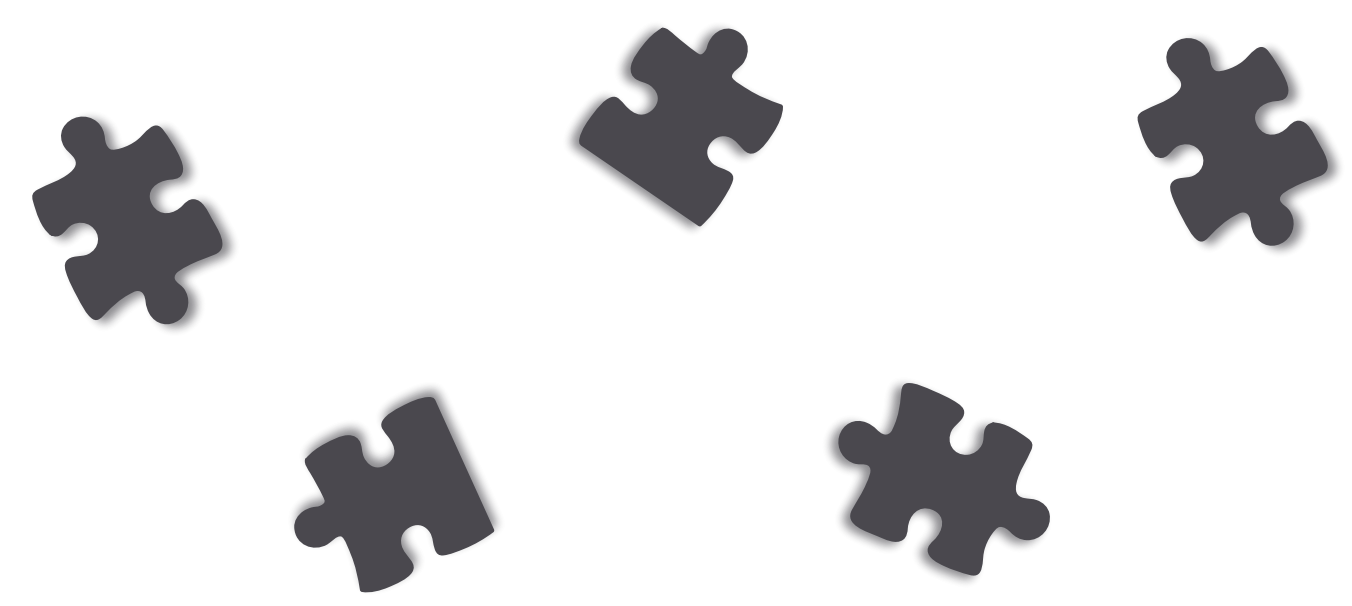

Brigitta Monz

Richard Houghton

Kiely Law

Georg Loss 


\begin{abstract}
Children with autism receive different types of non-drug treatments. We aimed to describe caregiver-reported pattern of care and its variability by geography and healthcare coverage in a US-wide sample of children aged 3-17 years. We recruited caregivers from the Simons Foundation Powering Autism Research for Knowledge (SPARK) cohort. Two online questionnaires (non-drug treatment, Autism Impact Measure) were completed in September/October 2017. Primary outcome measures were caregiver-reported types and intensities of treatments (behavioural, developmental/relationship, speech and language (SLT), occupational, psychological, "other"; parent/caregiver training) in the previous 12 months. Main explanatory variables were geography and type of healthcare coverage. We investigated associations between the type/intensity of treatments and geography (metropolitan/nonmetropolitan) or coverage (Medicaid vs privately insured by employer) using regression analysis. Caregivers ( $n=5,122)$ were mainly mothers $(92.1 \%)$ with mean (SD) age of 39.0 (7.3) years. Children had mean (SD) age 9.1 (3.9) years and were mostly males (80.0\%). Almost all children received at least one intervention (96.0\%). Eighty percent received SLT or occupational therapy, while 52.0\% received both. Behavioural therapy and SLT were significantly more frequent and more intense in metropolitan than in nonmetropolitan areas. No consistently significant associations were seen between healthcare coverage and frequency or intensity of interventions. At least one barrier such as "waiting list" and "no coverage" was reported by 44.8\%. In conclusion, in children sampled from SPARK, we observed differences between metropolitan and nonmetropolitan areas, while we did not find significant differences between those privately insured versus Medicaid.
\end{abstract}

\title{
Lay summary
}

The American Academy of Child and Adolescent Psychiatry recommends the use of multiple treatment modalities in autism spectrum disorder (ASD). We wanted to understand what types of treatment children (aged 3-17 years) with ASD receive in the United States, how and where the treatments take place and for how long. We invited caregivers from Simons Foundation Powering Autism Research for Knowledge ("SPARK"; https://sparkforautism.org/) to complete the study questions online. Participants reported on utilisation of conventional, non-drug treatments for ASD, including behavioural interventions, developmental/relationship interventions, speech and language therapy (SLT), occupational therapy, psychological therapy, and parent/caregiver training. People that completed the study ( $n=5,122)$ were primarily mothers of the child with ASD (92\%); most of the children were boys (80\%). The ASD care for the child was mostly coordinated by the mother. Almost all children received at least some type of non-drug therapies (96\%), most often SLT and/or occupational therapy, mainly provided in school. Behavioural therapy was most often received in public school in rural areas, while at home in urban areas. We saw less use of behavioural therapy and SLT in rural areas, but overall comparable use between children covered by Medicaid and those covered by private insurance. Almost half the caregivers reported at least one barrier to treatment, such as "waiting 
list" and "no coverage." More than half said that their child benefited "much" or "very much" from the therapies received. While overall non-drug treatment rates for children with ASD were high in the United States in our study, differences existed depending on where the family lives; not only regarding the type of therapy, but also where it takes place.

\section{Introduction}

Autism spectrum disorder (ASD) is a family of neurodevelopmental disorders characterised by repetitive or stereotyped behaviours and deficits in social interactions. An estimated 11.9 in 1,000 children in the United States (US) have ASD ${ }^{1}$. The American Academy of Child and Adolescent Psychiatry recommends the use of multiple treatment modalities in $\mathrm{ASD}^{2}$. Conventional non-drug treatments, including behavioural interventions, speech and language therapy (SLT), and occupational therapy (OT), are utilised across paediatric age groups and administered in diverse settings (e.g. home, school, and specialty clinics/ offices) ${ }^{3}$. Previous studies reporting on the pattern of care received by children with ASD either relied on a network of centres or providers ${ }^{4}$, utilised subsections of existing US surveys ${ }^{5}$, used claims analysis ${ }^{6}$, or investigated certain age groups ${ }^{7,8}$.

Prior research has raised concerns that children in more rural settings have access to fewer services ${ }^{9}$, as well as that the type of healthcare coverage may dictate utilisation of services, specifically that Medicaid provided for more interventions than commercial plans ${ }^{10}$. Therefore, the goal of our study was to describe the caregiver-reported pattern of non-drug ASD treatment and its variation by geographic region and type of healthcare coverage across the US in children aged 3-17 years. Our study recruited caregivers from the Simons Foundation Powering Autism Research for Knowledge (SPARK) cohort, a US-based online research cohort with individuals and families who have consented to providing information and medical samples to further autism research ${ }^{11}$.

\section{Methods}

Online surveys for non-drug therapy and for the Autism Impact Measure (AIM; to assess frequency and impact of ASD symptoms ${ }^{12,13}$ ) were sent to caregivers (i.e. parents and guardians/legally authorised representatives) in the SPARK cohort between 13th September and 22nd October 2017. Invitations were sent in four batches, first inviting potentially eligible caregivers (i.e. have children with ASD registered in SPARK in required age range) that had most recently joined SPARK. Participants and their oldest ASD dependent aged 3-17 years (hereafter "children with ASD") had to have been living in the same household, with the caregiver as the main person supporting this child for at least the preceding 12 months. SPARK currently provides information in English and requires for inclusion that participants be able to read and understand English. 
The research protocol was approved by an institutional review board (Western IRB) and participants consented online. Upon completion of both surveys, participants received a $\$ 20$ online shop voucher via email.

The primary outcome measures were the types and intensities of non-drug treatments in the preceding 12 months as reported by the caregiver, categorised into seven groups (child-directed: behavioural, developmental and/or relationship-based, SLT, OT, psychological, and "other"; and parent/caregiver training). This categorisation was similar to a study by Salomone et al ${ }^{14}$ in order to allow comparisons, with the only difference being that we separated out psychological interventions from the "other" category. The main explanatory variables were geography of residence and healthcare coverage type. Geography was defined by a six-level urban-rural classification scheme based on US state and county (i.e. six-level metropolitan statistical area (MSA) ${ }^{15}$ ). For the analysis of the association between geography and outcomes, we collapsed this to two levels (i.e. twolevel MSA), nonmetropolitan and metropolitan, indicating "rural" and "urban" as per the classification scheme. Healthcare coverage types were categorised into mutually exclusive groups: those with only Medicaid ("Medicaid-only"), those with only private insurance provided by an employer ("private insurance-only"), one other type of coverage, more than one, and uninsured. Barriers (adapted from a previous study ${ }^{16}$ ), caregiver's role in access to and perceived benefits of non-drug treatments, demographic characteristics of caregivers and children with ASD, and AIM scores were also analysed.

\section{Statistical Methods}

Data were summarised descriptively. Types and intensities of non-drug treatments were also stratified by geography, healthcare coverage type, and age group.

To model the associations between explanatory variables (geography, type of healthcare coverage) and outcomes, we identified covariates needed for adjustment using directed acyclic graphs ${ }^{17}$ (see supplement S.2.3.1 and online supplementary material: https://doi. org/10.1002/aur.2070), followed by propensity score methodology (inverse probability weighting) to create balance in the covariates, and finally applied regression modelling (logistic for treatment types; negative binomial for intensities). Populations did not sufficiently overlap to allow modelling the association between all four categories of insurance simultaneously, using multinomial logistic regression to derive propensity scores (i.e. Medicaid, private provided by employer, one type of coverage from the other categories, more than one coverage type). We therefore present only a comparison between private via employer versus Medicaid.

An intervention was counted as "absent" if a response of "don't know" was given. For present treatments, missing intensity values were set to $0.5 \mathrm{hr} /$ week. These imputations were necessary $<5 \%$ of the time as the data were generally very well populated. For AIM, no total or domain scores were calculated if $>20 \%$ of items had missing responses. 
We justified the sample size target of 5,000 based on the following: if $85 \%$ of children received at least one non-drug treatment and up to 16 strata analysed, a precision of 0.85 (95\% confidence interval [Cl]: 0.81-0.89) could be achieved, which was considered adequate. R version 3.3.2 was used for all analyses.

\section{Results}

Invitations were emailed to 11,514 of 19,142 potentially eligible caregivers. The non-drug treatment survey was completed by 5,122 (44.5\% of those invited), and the AIM by 5,001 (43.4\%; see supplement S.2.3.2). The study was closed online when the targeted sample size had been reached.

\section{Characteristics of caregivers and children with ASD}

The majority (92.1\%) of the caregivers were mothers, with a mean age of 39.0 years and were mostly (76.5\%) White/non-Hispanic (Table 2.3.1). Two-thirds had a higher education (completed college or higher) and most (81.2\%) lived in metropolitan areas. The children with ASD were predominantly (80.0\%) male, with a mean age of 9.1 years, and mostly (68.5\%) White/non-Hispanic. About two-thirds had been diagnosed before age 5 years. Almost all had at least some insurance coverage. Of those who reported having ever had an IQ test, $44.6 \%$ scored $<100$.

There were a few demographic differences between children enrolled in special-education schools (21.7\% of the total) and the overall group; notably, mean age was lower, a lower proportion were of White/non-Hispanic ethnicity, a higher proportion had been diagnosed before 5 years, and had IQ test scores of 70 or below (see online supplementary material: https://doi.org/10.1002/aur.2070).

The mean (SD) total AIM score was 220.8 (54.1); possible score range: 82-410, with higher scores indicating higher symptom frequency/impact. Mean (SD) [possible range] domain scores were: Repetitive Behavior, 41.1 (13.8) [16-80]; Communication, 30.6 (11.9) [12-60]; Atypical Behavior, 34.7 (10.1) [12-60]; Social Reciprocity 27.1 (7.4) [10-50]; and Peer Interaction, 22.9 (7.1) [8-40]. 
Table 2.3.1: Characteristics of caregivers and their children with ASD

\begin{tabular}{|c|c|c|}
\hline & Characteristic & $\begin{array}{c}\text { Number (\%) } \\
\text { [except where } \\
\text { indicated otherwise] }\end{array}$ \\
\hline \multicolumn{3}{|l|}{ Caregivers } \\
\hline & Age, years [mean (SD)] & $39.02(7.30)$ \\
\hline & Relation to child & \\
\hline & Mother & $4719(92.1)$ \\
\hline & Father & $314(6.1)$ \\
\hline & Legal guardian & $55(1.1)$ \\
\hline & Other & $34(0.7)$ \\
\hline & Married/living with partner & $4101(80.1)$ \\
\hline & $\begin{array}{l}\text { Completed college or higher } \\
\text { Employment }\end{array}$ & 3259 (63.6) \\
\hline & Working (full or part-time) & $3024(59.0)$ \\
\hline & Full-time homemaker & $1541(30.1)$ \\
\hline & Other & $557(10.9)$ \\
\hline & Race/ethnicity & \\
\hline & White/Non-Hispanic & $3919(76.5)$ \\
\hline & White/Hispanic & 301 (5.9) \\
\hline & Non-white/Non-Hispanic & $650(12.7)$ \\
\hline & Non-white/Hispanic & $252(4.9)$ \\
\hline & $\begin{array}{l}\text { More than one child with autism in family } \\
\text { Region }\end{array}$ & $801(15.6)$ \\
\hline & Region & $1297(25.3)$ \\
\hline & Midwest & $1124(21.9)$ \\
\hline & Northeast & $825(16.1)$ \\
\hline & South & $1868(36.5)$ \\
\hline & Unknown & $8(0.2)$ \\
\hline & Metropolitan Statistical Area & \\
\hline & Metropolitan & $4158(81.2)$ \\
\hline & Nonmetropolitan & $588(11.5)$ \\
\hline & Unknown & $376(7.3)$ \\
\hline & Self-reported health & \\
\hline & Excellent & $838(16.4)$ \\
\hline & Very Good & $2049(40.0)$ \\
\hline & Good & 1699 (33.2) \\
\hline & Fair & $448(8.7)$ \\
\hline & Poor & $82(1.6)$ \\
\hline & Missing & $6(0.1)$ \\
\hline & Household income & \\
\hline & Less than $\$ 20,000$ & $512(10.0)$ \\
\hline & $\$ 20,000-\$ 34,999$ & $696(13.6)$ \\
\hline & $\$ 35,000-\$ 49,999$ & $619(12.1)$ \\
\hline & $\$ 50,000-\$ 74,999$ & 904 (17.6) \\
\hline & $\$ 75,000-\$ 99,999$ & 681 (13.3) \\
\hline & $\$ 100,000-\$ 124,999$ & $577(11.3)$ \\
\hline & $\$ 125,000-\$ 149,999$ & $312(6.1)$ \\
\hline & $\$ 150,000$ or more & $576(11.2)$ \\
\hline & Missing & $245(4.8)$ \\
\hline
\end{tabular}


Table 2.3.1: Characteristics of caregivers and their children with ASD (cont.)

\begin{tabular}{|c|c|c|}
\hline & Characteristic & $\begin{array}{c}\text { Number (\%) } \\
\text { [except where } \\
\text { indicated otherwise] }\end{array}$ \\
\hline \multicolumn{3}{|l|}{ Children with ASD } \\
\hline & Age, years [mean (SD)] & $9.10(3.92)$ \\
\hline & Male & $4096(80.0)$ \\
\hline & Race/ethnicity & \\
\hline & White/Non-Hispanic & $3510(68.5)$ \\
\hline & White/Hispanic & $501(9.8)$ \\
\hline & Non-white/Non-Hispanic & $830(16.2)$ \\
\hline & Non-white/Hispanic & $281(5.5)$ \\
\hline & Caregiver-reported child's health & \\
\hline & Excellent & $1694(33.1)$ \\
\hline & Very Good & $2174(42.4)$ \\
\hline & Good & $1063(20.8)$ \\
\hline & Fair & 169 (3.3) \\
\hline & Poor & $13(0.3)$ \\
\hline & Missing & $9(0.2)$ \\
\hline & Autism diagnosis & \\
\hline & ASD & $3786(73.9)$ \\
\hline & Autism/Autistic Disorder & $553(10.8)$ \\
\hline & Asperger Syndrome & $455(8.9)$ \\
\hline & PDDNOS & $289(5.6)$ \\
\hline & Unknown/Missing & $39(0.8)$ \\
\hline & Age at autism diagnosis, years & \\
\hline & $0-2$ & $1337(26.1)$ \\
\hline & $3-4$ & $1999(39.0)$ \\
\hline & $5-9$ & $1418(27.7)$ \\
\hline & $>9$ & $348(6.8)$ \\
\hline & Missing & $20(0.4)$ \\
\hline & Years since autism diagnosis & \\
\hline & $0-1$ & $1093(21.3)$ \\
\hline & $2-3$ & $1303(25.4)$ \\
\hline & $4-5$ & $883(17.2)$ \\
\hline & $6-9$ & $1130(22.1)$ \\
\hline & $\geq 10$ & $697(13.6)$ \\
\hline & Missing & $16(0.3)$ \\
\hline & Insurance & \\
\hline & Uninsured/unknown & $87(1.7)$ \\
\hline & Only Medicaid & $1564(30.5)$ \\
\hline & Only private (via employer) & $2083(40.7)$ \\
\hline & One type of other coverage & $418(8.2)$ \\
\hline & More than one type & $970(18.9)$ \\
\hline & Insurance drug coverage & $4672(91.2)$ \\
\hline & Prescription drug use & \\
\hline & Overall & $2683(52.4)$ \\
\hline & Drugs for autism & $1718(33.5)$ \\
\hline
\end{tabular}


Table 2.3.1: Characteristics of caregivers and their children with ASD (cont.)

\begin{tabular}{|c|c|}
\hline Characteristic & $\begin{array}{c}\text { Number (\%) } \\
\text { [except where } \\
\text { indicated otherwise] }\end{array}$ \\
\hline \multicolumn{2}{|l|}{ Over-the-counter drug use } \\
\hline Overall & $3106(60.6)$ \\
\hline Drugs for autism & 965 (18.8) \\
\hline Has other medical problems & $2348(45.8)$ \\
\hline Has other mental health or psychiatric problems & $2424(47.3)$ \\
\hline $\begin{array}{l}\text { Primary care physician main healthcare provider } \\
\text { IQ test results* }\end{array}$ & $2911(56.8)$ \\
\hline$\leq 70$ & $464(20.1)$ \\
\hline 71-99 & $564(24.5)$ \\
\hline$\geq 100$ & $789(34.2)$ \\
\hline Unknown & $488(21.2)$ \\
\hline Attending school with special education students only & 1109 (21.7) \\
\hline At least $60 \%$ of classroom time with typically-developing peers & $2312(45.1)$ \\
\hline
\end{tabular}

$\mathrm{n}=5122$ is the number of caregiver respondents and ASD children (oldest eligible autistic child considered per respondent).

ASD: Autism Spectrum Disorder; IQ: intelligence quotient; PDDNOS: pervasive developmental disorder - not otherwise specified; SD: standard deviation.

${ }^{*}$ IQ test was previously completed by $n=2305$ (45.0\%).

\section{Types of treatments}

As shown in Table 2.3.2, 96.0\% of children received at least one type of non-drug treatment, the most common being SLT (71.4\%). A higher proportion of children in metropolitan versus nonmetropolitan areas received behavioural therapy $(57.2 \%$ vs 46.4\%) and SLT (72.3\% vs 65.0\%). There was a pattern toward lower utilisation across urbanisation categories from large central metro to noncore for behavioural therapy, SLT, and parent/caregiver training (see supplement S.2.3.3). Similar proportions of children covered by Medicaid-only and private insurance-only received at least one therapy $(96.2 \%$ vs 95.4\%), although children under Medicaid-only received OT more frequently $(61.8 \%$ vs 55.7\%) and "other" therapies less frequently (63.4\% vs 69.9\%). For most therapies, use decreased from the lowest age group to the highest age group.

Most caregivers reported therapy as ongoing (i.e. not having ended in the 12-month recall period; ranging from 61.0\% for parent/caregiver training to 89.1\% for SLT). Approximately $42 \%$ reported four or more interventions. Children were most likely to receive SLT and OT concurrently, followed by parent/caregiver training and behavioural therapy (supplement S.2.3.4). The most common interventions used concurrently were behavioural-SLT-OT-other (7.1\%) and SLT-OT-other (5.9\%; see supplement S.2.3.5). In nonmetropolitan areas, behavioural therapy was not in the three most common concurrently used interventions, and developmental/ relationship-based and parental training not in any of the combinations occurring for $>2 \%$ of the children. Overall, 52.0\% received at least SLT and OT, while 79.5\% received at least either. 


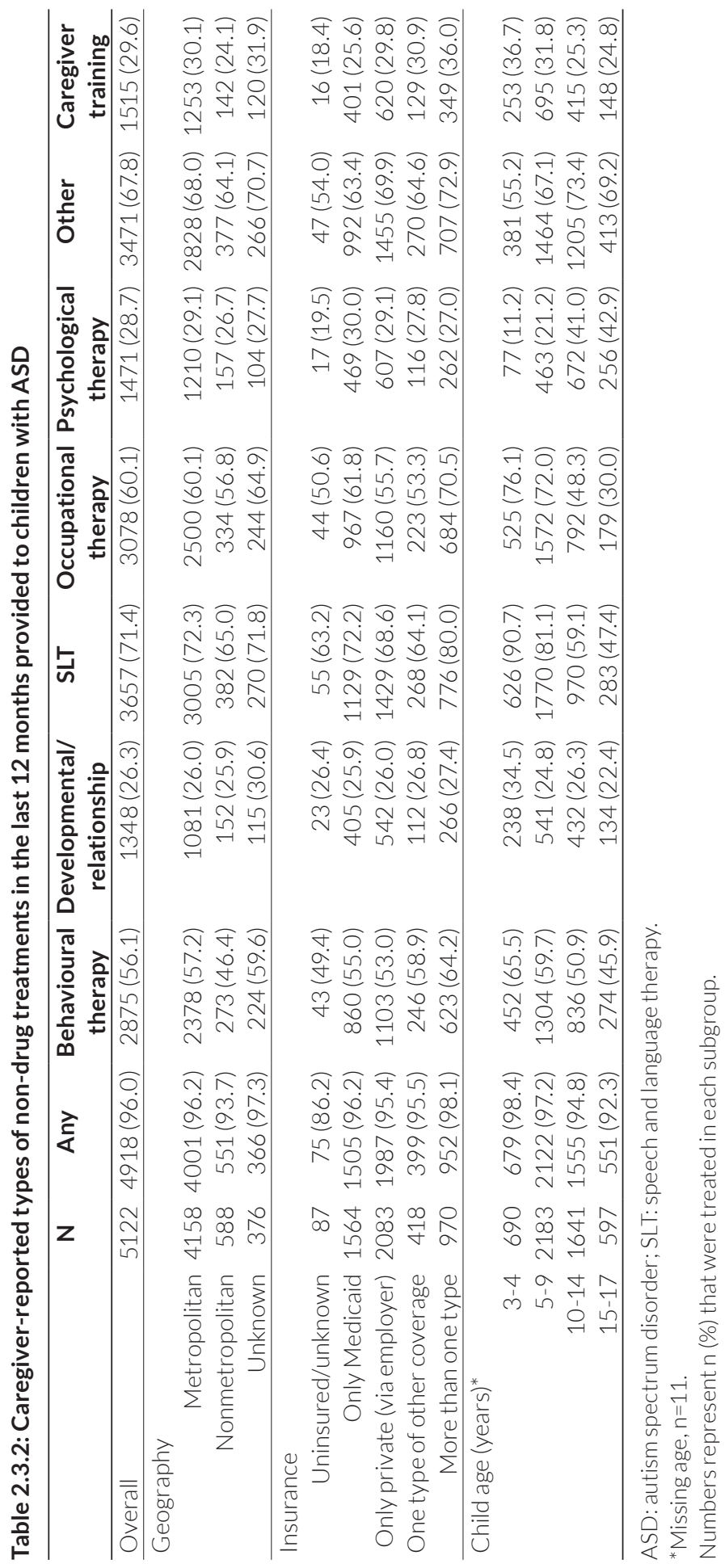




\section{Intensity of treatments}

The median intensity of all treatments was $6.0 \mathrm{hr} /$ week, with behavioural therapy being the most intense (4.0 hr/week; Table 2.3.3). Metropolitan areas reported higher intensity for "any" therapy, behavioral therapy, "other" therapy, and developmental/relationshipbased interventions (supplement S.2.3.6). A difference between Medicaid-only and private insurance-only patients was seen only for behavioral therapy (2.0 and $4.0 \mathrm{hr}$ / week, respectively) and "other" (supplement S.2.3.7). Although there was no consistent pattern overall across age groups, the two lowest (3-4 and 5-9 years) had the highest intensity for behavioural therapy (Table 2.3.3).

\section{Setting of treatments}

Non-drug therapies were more often given in individual rather than group sessions, with psychological interventions and OT having the highest individual-to-group ratios (IGRs: 3.27 and 2.93, respectively; Table 2.3.3). Children in nonmetropolitan areas were more likely to receive individual sessions than those in metropolitan areas, with notable differences seen for behavioural therapy, psychological interventions, OT, and SLT. Children under Medicaid-only more often received individual sessions than those under private insurance-only; with differences seen for OT, SLT, and behavioural therapy. There was a pattern of individual sessions from lowest in large central metro to highest in noncore (supplement S.2.3.8).

SLT and OT were more often provided in school (school/not in school ratio [SNR]: 1.79 and 1.33, respectively), while behavioural therapy and psychological interventions were more frequently provided outside school (SNR: 0.64 and 0.40, respectively). There were no notable differences in SNR's between metropolitan and nonmetropolitan areas. A small difference was seen between the Medicaid-only and private insurance-only patients for SLT.

The most common place of care was the home for behavioural interventions (45.0\%); public school for developmental/relationship-based interventions (56.0\%), SLT (76.5\%), OT (63.6\%) and "Other" interventions (57.7\%); and private therapist (57.8\%) for psychological interventions (Table 2.3.4). Behavioural therapy was most often received in public school in nonmetropolitan areas (44.7\%), but at home in metropolitan areas (46.3\%).

\section{Barriers to treatments}

Overall, 44.8\% reported at least one barrier to non-drug therapy. "Waiting list" (26.4\%) was the most common provider-related barrier (Table 2.3.5), whereas "no coverage" (17.9\%) and "cost" (16.7\%) were the most common health-plan-related barriers (Table 2.3.6). Metropolitan areas reported a higher frequency of "waiting list" than nonmetropolitan areas, but a much lower frequency of "not available in area" (15.1\% vs $32.0 \%$ ). 


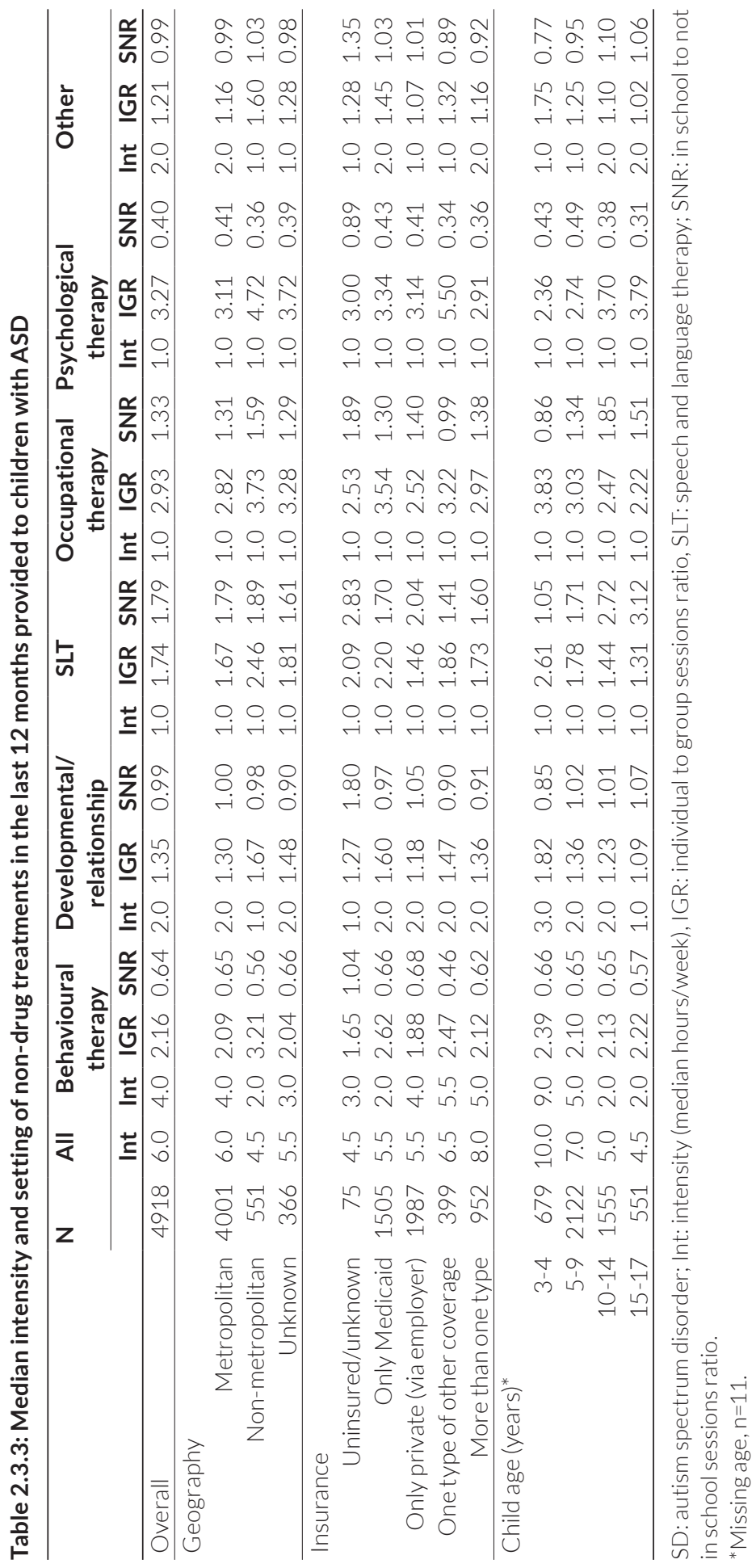




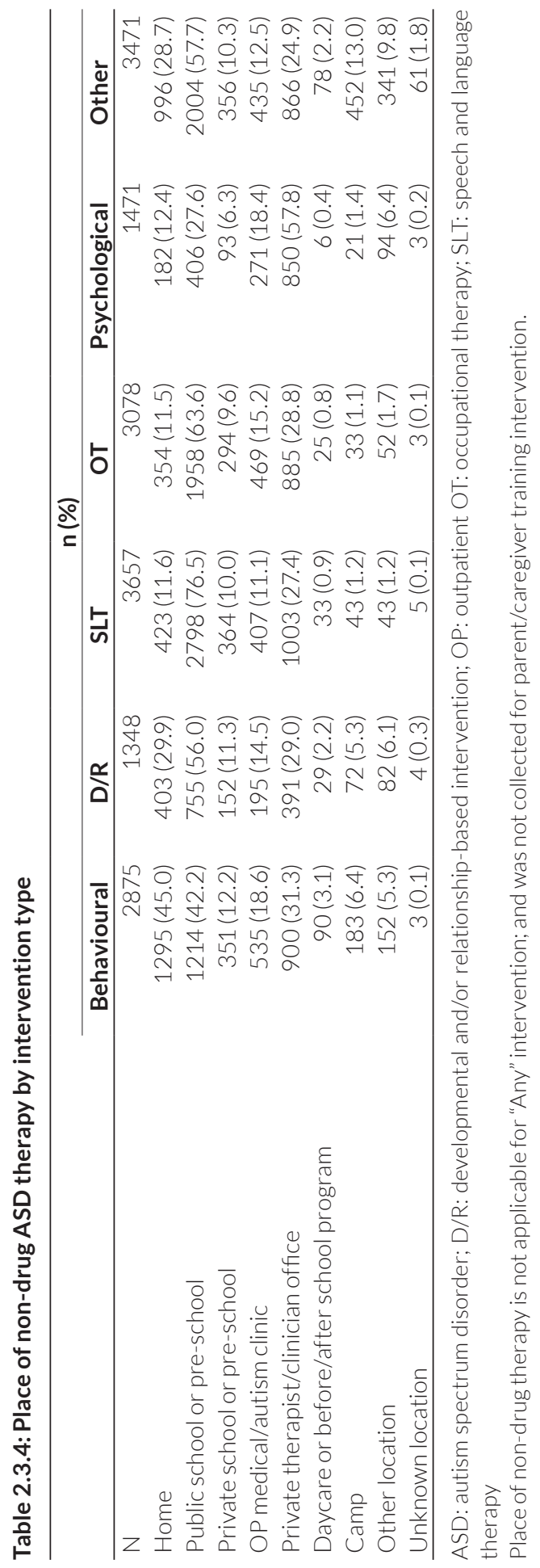




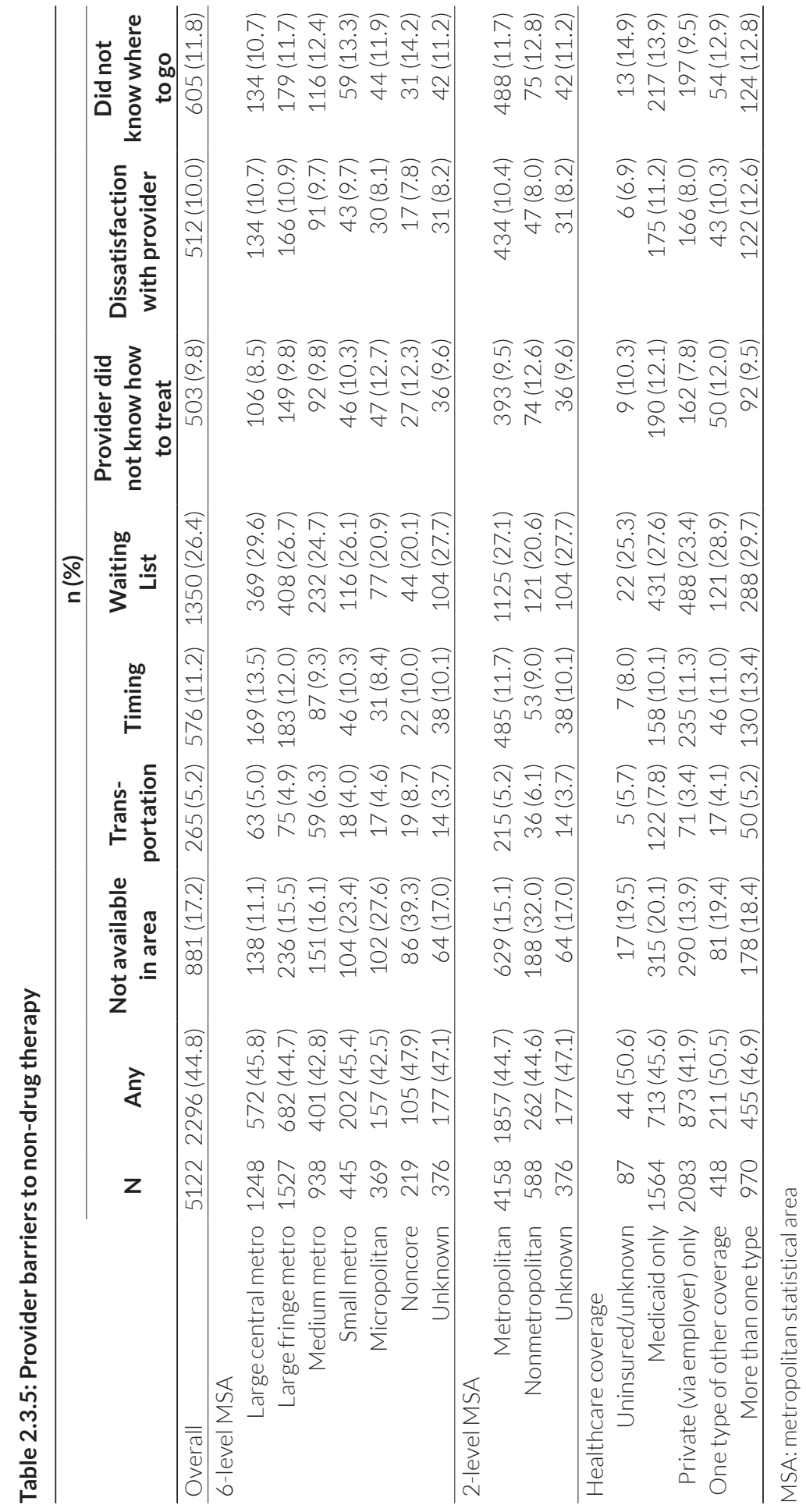




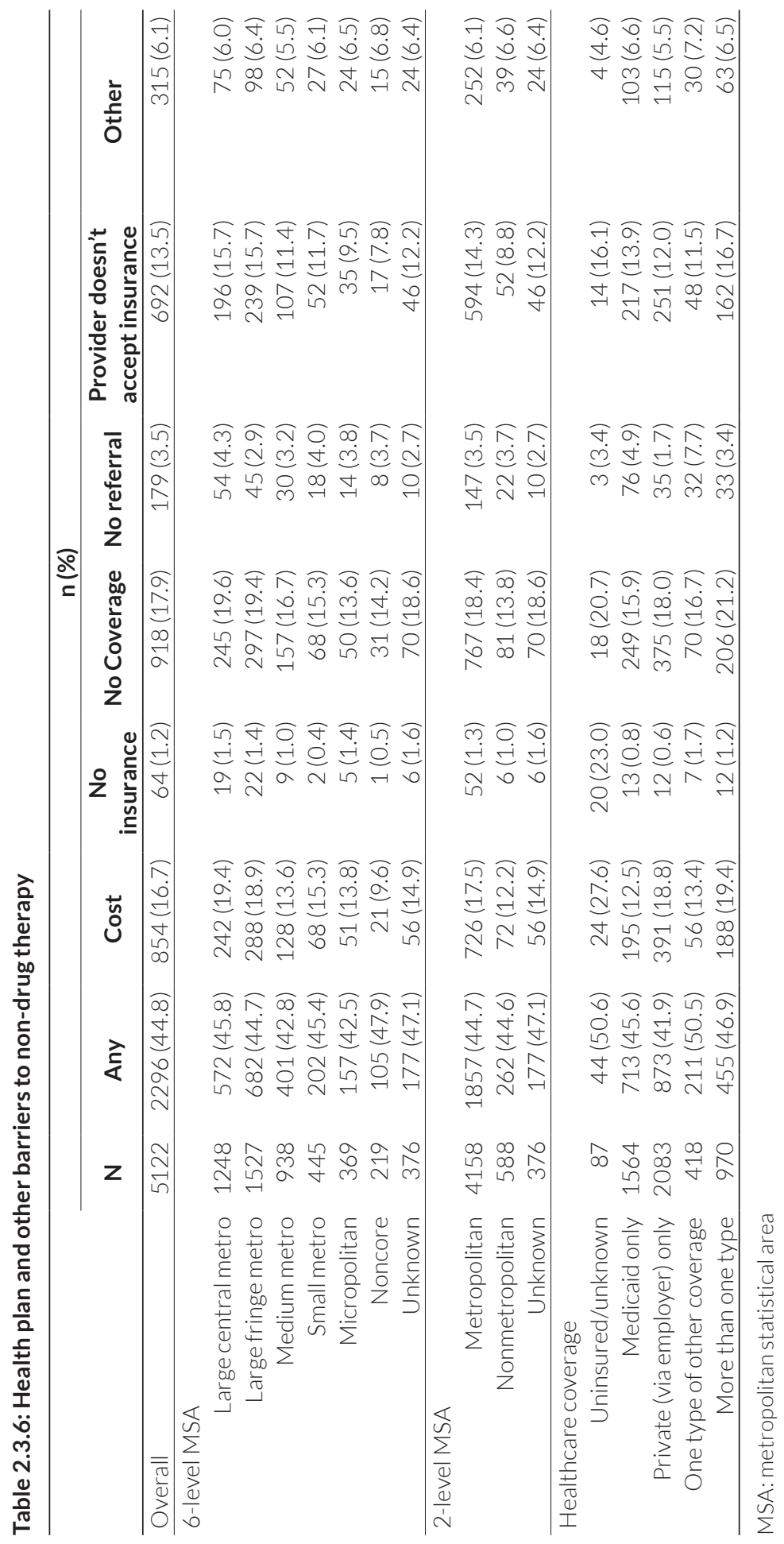




\section{Caregiver's role and perception}

Table 2.3.7 lists the non-drug therapies in the "other" category. The most frequent therapies were social skills training (37.0\%) and academic support (28.3\%). The main care coordinator was the caregiver (81.9\%). Three quarters reported that they were satisfied with the current level of care, and 58.2\% reported that their child benefited "much" or "very much" from care.

\section{Associations between treatments and geography/insurance}

As shown in Table 2.3.8, the odds of receiving "any" treatment, behavioural therapy and SLT were significantly greater in metropolitan areas than in nonmetropolitan areas (odds ratios [OR]: 1.71, 1.54, and 1.41, respectively). No consistently (i.e., significant in both, the PSadjusted and double-adjusted analyses) significant associations were seen between type of insurance (Medicaid-only vs private insurance-only) and type of treatment received.

The intensity of "any" treatment was significantly greater in metropolitan areas than in nonmetropolitan areas (rate ratio [RR]: 1.35), based on the intensities of behavioural therapy, SLT, psychological and "other" interventions that were all significantly greater in metropolitan areas. The RRs for the intensity of any of the treatment options did not indicate a consistently significant difference between the two types of healthcare coverage, except for "other," where the rates were lower for those with private insurance provided by employer. For results on the six-level MSA and other additional analyses, refer to the supplements S.2.3.9 to S.2.3.11.

Table 2.3.7: Non-drug ASD therapies in the "other" category

\begin{tabular}{lrr}
\hline Type & $\mathbf{n}$ & \% \\
\hline Social skills training & 1894 & 36.98 \\
Academic support (for example reading, writing, and math tutor) & 1450 & 28.31 \\
Sensory integration & 792 & 15.46 \\
Physical therapy & 739 & 14.43 \\
Recreational* & 715 & 13.96 \\
Biomedical** & 431 & 8.41 \\
Animal-assisted activities and therapies*** & 395 & 7.71 \\
Other therapy not previously mentioned & 395 & 7.71 \\
Other therapy - but don't know which & 234 & 4.57 \\
Fast Forward, APE & 206 & 4.02 \\
Structured Teaching (TEACCH) & 177 & 3.46 \\
SCERTS & 152 & 2.97 \\
AIT & 78 & 1.52 \\
The Built Environment & 2 & 0.04 \\
\hline
\end{tabular}

Percentages are based on the 3,471 children who received at least one "other" therapy.

AIT: auditory integration training/therapy; APE: Adaptive physical education; ASD: Autism Spectrum Disorder; SCERTS: Social Communication/Emotional Regulation/Transactional Support; TEACCH:

Training and Education of Autistic and Related Communication Handicapped Children

*For example, specialised summer camp

**For example, biofeedback, special diets, vitamins

***For example, therapies including dogs, horses, dolphins 
Table 2.3.8: Association of caregiver-reported type and intensity of non-drug therapy with geography and insurance

\begin{tabular}{|c|c|c|c|}
\hline \multirow{2}{*}{$\begin{array}{l}\text { Non-drug } \\
\text { therapy type }\end{array}$} & \multirow[t]{2}{*}{$\begin{array}{c}\text { Geography } \\
\text { (Metropolitan } \\
\text { vs Nonmetropolitan) } \\
\mathrm{N}=4,746 \\
\text { Unadjusted }\end{array}$} & \multicolumn{2}{|c|}{$\begin{array}{c}\text { Insurance } \\
\text { (Only private via employer } \\
\text { vs only Medicaid) } \\
\mathrm{N}=1,632\end{array}$} \\
\hline & & PS-adjusted & Double-adjusted \\
\hline & \multicolumn{3}{|c|}{ Association with type of therapy: OR $(95 \% \mathrm{Cl})$} \\
\hline Any & $1.71(1.17,2.45)$ & $0.63(0.34,1.17)$ & $0.58(0.32,1.06)$ \\
\hline Behavioural & $1.54(1.30,1.83)$ & $1.12(0.78,1.62)$ & $1.07(0.78,1.47)$ \\
\hline Developmental/ relationship & $1.01(0.83,1.23)$ & $0.99(0.65,1.52)$ & $1.04(0.7$ \\
\hline SLT & 1.69) & $0.67(0.44,1.03)$ & $0.97)$ \\
\hline Occupational & $1.15(0.9$ & $0.80(0.54,1.19)$ & $0.77(0.5$ \\
\hline Psychological & 1.13 & $0.77(0.55,1.09)$ & .97) \\
\hline \multirow[t]{2}{*}{ Other } & $1.19(0.99,1.42)$ & $0.86(0.58,1.26)$ & $0.86(0.62,1.20)$ \\
\hline & \multicolumn{3}{|c|}{ Association with intensity of therapy: $\mathrm{RR}(95 \% \mathrm{CI})$} \\
\hline Any & $1.35(1.23,1.48)$ & $0.90(0.75,1.07)$ & $0.84(0.72,0.97)$ \\
\hline Behaviou & $1.71(1.45,2.01)$ & $1.02(0.81,1.27)$ & $0.98(0.78,1.24)$ \\
\hline Developmental/ relationship & $1.23(0.95,1.57)$ & $1.37(0.93,2.01)$ & $0.99(0.70,1.39)$ \\
\hline SLT & $1.18(1.06,1.31)$ & $0.90(0.70,1.17)$ & $0.88(0.72,1.07)$ \\
\hline Occupationa & $1.04(0.93,1.17)$ & $0.83(0.64,1.08)$ & $0.83(0.66,1.05)$ \\
\hline Psychological & $1.46(1 \cdot 19,1.79)$ & $0.89(0.60,1.32)$ & $0.81(0.56,1.17)$ \\
\hline Other & $1.19(1.04,1.35)$ & $0.66(0.54,0.81)$ & $0.65(0.54,0.80)$ \\
\hline
\end{tabular}

Cl: Confidence interval; OR: Odds Ratio; PS: propensity score; RR: Rate ratio; SLT: Speech and language therapy

Models for insurance were adjusted for below variables using propensity score inverse probability weighting (adjusted and double adjusted: see methods section). See supplement S.2.3.9 for trimmed results.

Variables for adjustment: AIM domain scores (continuous), child race/ethnicity (White/Hispanic, White/ Non-Hispanic, Non-White/Hispanic, Non-White/Non-Hispanic), child other medical problems (yes/no), child other mental health or psychiatric problems (yes/no), geography (nonmetropolitan/ metropolitan), household income (four strata, $\geq \$ 20,000$ to $\leq \$ 99,999$ ), marital status (married/living with partner yes/no), mother employment (work full time/part time yes/no), US state (excluded states with $\mathrm{n}<3$ for Medicaid and private employer insurance).

\section{Discussion}

This study investigated the caregiver-reported pattern of non-drug therapy and the variability of care by geographic region and type of healthcare coverage, in children with ASD.

The cohort was largely representative of children with ASD in the US. Population weighted survey data, from the 2016 National Survey of Children's Health ${ }^{18}$, reported a similar male to female ratio (around $80 \%$ male), use of medications for autism (around $1 / 3$ ), age at diagnosis (around 1/3 beyond age 5 years), and insurance coverage (around 98\% with coverage). The sample of the 2016 survey included close to $70 \%$ White/nonHispanics, as in our study, while the population estimate was around 53\%, indicating overrepresentation of this group among the survey respondents. 
Salomone et al ${ }^{14}$ who grouped therapies similarly, reported that $91 \%$ of respondents from 18 European countries used at least one non-drug therapy (vs 96\% in our study). Behavioural therapy, SLT, and OT were reported at 32\%, 64\%, and 35\%, although there was wide variation across countries. Our study reported higher rates (66\% and 60\%, $91 \%$ and $81 \%, 76 \%$ and $72 \%$ for these therapies, for the age groups $3-4$ years and 5-9 years, respectively, corresponding best to the age range investigated by Salomone et al). Most children in our cohort received more than one non-drug therapy. Approximately one-quarter used three therapies concurrently and two-thirds used three or more in the past 12 months with the most common combination being behavioural-SLT-OT-other (7.1\%). It is difficult to compare these rates with other studies as they either did not use similar groupings of non-drug treatments, included medications and other modalities (e.g. vitamin supplements) in their count of combinations, or did not report on combinations at all. Guideline-recommended intensity of treatment of $25 \mathrm{hr} /$ week for children ${ }^{19}$, was not achieved in our study. Even the most intense treatments have not reached this level (children aged 3-4 years, four or more different interventions, mean intensity $19.7 \mathrm{hr}$ / week).

SLT was the most frequently used therapy in our study and was predominantly provided at public schools. That SLT was the most frequent therapy agrees with previous findings in the US (from a 2012 survey of four Kaiser Permanente regions ${ }^{4}$ ) and in Europe ${ }^{14}$. Since communication deficits are a core symptom of ASD, the high rate of SLT is not surprising.

We classified geography based on state and county, defining six levels of urbanisation from most urban/highest density to most rural/lowest density ${ }^{15}$. The difference in frequency of "any" treatment between metropolitan and nonmetropolitan areas (96.2\% vs $93.7 \%$, OR 1.71 [95\% Cl: 1.17, 2.45]), although significant, was less pronounced than might have been expected ${ }^{9}$. This may be because the most frequently used therapies (SLT and OT) are mostly provided at public school, and are thus not dependent on infrastructure such as specialists' offices. The intensity of "any" treatment was also significantly greater in metropolitan areas than in nonmetropolitan areas, mostly driven by significant differences in the intensity of behavioural therapy, SLT, psychological, and "other" interventions. Specifically, behavioural therapy was less often used in nonmetropolitan settings, and, where used, was much less intense. Given that public school was reported as the most frequent setting for this therapy in nonmetropolitan areas, its use as a platform for care delivery probably warrants further consideration. A previous study ${ }^{9}$ additionally suggested telehealth programs to provide better access to behavioural therapy in remote areas. The level of urbanisation has an influence on the number of children diagnosed with ASD ${ }^{20}$, also called "treated prevalence" ${ }^{21}$. Hence, the true difference in unmet need between metropolitan and nonmetropolitan areas is likely underestimated in our study.

Historically, large differences have been reported for ASD-related services between children covered under Medicaid versus those under private insurance, such that 
Medicaid spending was much higher ${ }^{10,22}$. We were able to investigate the association between frequency/intensity and type of healthcare coverage in two mutually exclusive insurance groups, namely Medicaid-only and private employer-based-only. We made these groups as comparable as possible by focusing on a subgroup of children with similar characteristics and further adjusting for important confounders. No significant differences in the frequency or intensity of treatment was observed, with the exception that intensity for "other" interventions was lower in privately insured children. However, given that the sample size for this analysis was substantially smaller than for the analysis of association with geography, the results may also reflect limited statistical power, as point estimates mostly directionally favoured Medicaid, except for behavioural and developmental-relationship based therapies.

Our study has some limitations. The use of the SPARK cohort might have introduced selection bias toward caregivers with higher motivation and higher education. This is likely to have underestimated the difference between nonmetropolitan and metropolitan areas, given the known associations between area of residence and educational level and likelihood of seeking treatment ${ }^{8}$. The possibility of recall bias cannot be ruled out as the study relied on caregiver-reported information over the previous year. However, as most of the treatments were still ongoing, this seems to be less of a concern. Our grouping of treatment options into categories may not be universally acceptable, although a very similar grouping has been used before ${ }^{14}$, and caregivers may not have been able to clearly identify and distinguish the different treatment options. We also did not collect in the "other" category further details for the response option "other therapy not previously mentioned" which may include those considered complementary health approaches. Finally, as this was a cross-sectional study, it is not possible to conclude any causal relationships, particularly between geographic region and any type of healthcare coverage, although it is implied that the pattern of care is determined by geography of residence and type of coverage.

Strengths of this study lie in the recency of the data presented (2016 and 2017), and its collection from a large sample not linked to a specific provider or network of centres, and no reliance on claims data. Since most respondents reported themselves as the main care coordinator, the data are likely to be complete. The design of the survey also allowed us to capture important details including the setting of care. Finally, these data will be made available via SPARK, and will be linkable to other data collected from the same cohort.

While this study provides unprecedented detail on current non-drug therapy in ASD for children in the US, future research should investigate the effectiveness of those treatments in routine practice. 


\section{References}

1. Durkin, M. S. et al. Autism spectrum disorder among US children (20022010): Socioeconomic, racial, and ethnic disparities. Am. J. Public Health 107, 1818-1826 (2017).

2. Volkmar, F. et al. Practice parameter for the assessment and treatment of children and adolescents with autism spectrum disorder. J. Am. Acad. Child Adolesc. Psychiatry 53, 237-257 (2014).

3. Nguyen, C. T., Krakowiak, P., Hansen, R., Hertz-Picciotto, I. \& Angkustsiri, K. Sociodemographic Disparities in Intervention Service Utilization in Families of Children with Autism Spectrum Disorder. J. Autism Dev. Disord. 46, 3729-3738 (2016).

4. Becerra, T. A. et al. A Survey of Parents with Children on the Autism Spectrum: Experience with Services and Treatments. Perm. J. 21, 16-009 (2017).

5. Vohra, R., Madhavan, S., Sambamoorthi, U. \& St Peter, C. Access to services, quality of care, and family impact for children with autism, other developmental disabilities, and other mental health conditions. Autism Int. J. Res. Pract. 18, 815-826 (2014).

6. Candon, M. K. et al. The Differential Effects of Insurance Mandates on Health Care Spending for Children's Autism Spectrum Disorder. Med. Care 56, 228232 (2018).

7. Zuckerman, K., Lindly, O. J. \& Chavez, A. E. Timeliness of Autism Spectrum Disorder Diagnosis and Use of Services Among U.S. Elementary School-Aged Children. Psychiatr. Serv. Wash. DC 68, 33-40 (2017).

8. Payakachat, N., Tilford, J. M. \& Kuhlthau, K. A. Parent-Reported Use of Interventions by Toddlers and Preschoolers With Autism Spectrum Disorder. Psychiatr. Serv. Wash. DC 69, 186-194 (2018).

9. Kelleher, K. J. \& Gardner, W. Out of Sight, Out of Mind - Behavioral and Developmental Care for Rural Children. N. Engl. J. Med. 376, 1301-1303 (2017).

10. Wang, L., Mandell, D. S., Lawer, L., Cidav, Z. \& Leslie, D. L. Healthcare service use and costs for autism spectrum disorder: a comparison between medicaid and private insurance. J. Autism Dev. Disord. 43, 1057-1064 (2013).
11. SPARK Consortium. SPARK:A US Cohort of 50,000 Families to Accelerate Autism Research. Neuron 97, 488-493 (2018).

12. Kanne, S. M. et al. The Autism Impact Measure (AIM): initial development of a new tool for treatment outcome measurement. J. Autism Dev. Disord. 44, 168-179 (2014).

13. Mazurek, M. O. et al. Construct Validity of the Autism Impact Measure (AIM). J. Autism Dev. Disord. 50, 2307-2319 (2020).

14. Salomone, E. etal. Use of early intervention for young children with autism spectrum disorder across Europe. Autism Int. J. Res. Pract. 20, 233-249 (2016).

15. Ingram, D. D. \& Franco, S. J. 2013 NCHS Urban-Rural Classification Scheme for Counties. Vital Health Stat. 2. 1-73 (2014).

16. Chiri, G. \& Warfield, M. E. Unmet need and problems accessing core health care services for children with autism spectrum disorder. Matern. Child Health J. 16, 1081-1091 (2012).

17. Greenland, S., Pearl, J. \& Robins, J. M. Causal diagrams for epidemiologic research. Epidemiol. Camb. Mass 10, 37-48 (1999).

18. Data Resource Center for Child \& Adolescent Health. National Survey of Children's Health (2016 - present). https://www.childhealthdata.org/ browse/survey (2018).

19. Maglione, M. A. et al. Nonmedical interventions for children with ASD: recommended guidelines and further research needs. Pediatrics 130 Suppl 2, S169-178 (2012).

20. Antezana, L., Scarpa, A., Valdespino, A., Albright, J. \& Richey, J. A. Rural Trends in Diagnosis and Services for Autism Spectrum Disorder. Front. Psychol. 8, 590 (2017).

21. Mandell, D. S. et al. Effects of Autism Spectrum Disorder Insurance Mandates on the Treated Prevalence of Autism Spectrum Disorder. JAMA Pediatr. 170, 887-893 (2016).

22. Zhang, W. \& Baranek, G. The Impact of Insurance Coverage Types on Access to and Utilization of Health Services for U.S. Children With Autism. Psychiatr. Serv. 67, 908-911 (2016) 
S.2.3.1: Directed acyclic graph displaying the relationship between non-drug treatment, covariates, and key exposures (insurance type and geography)

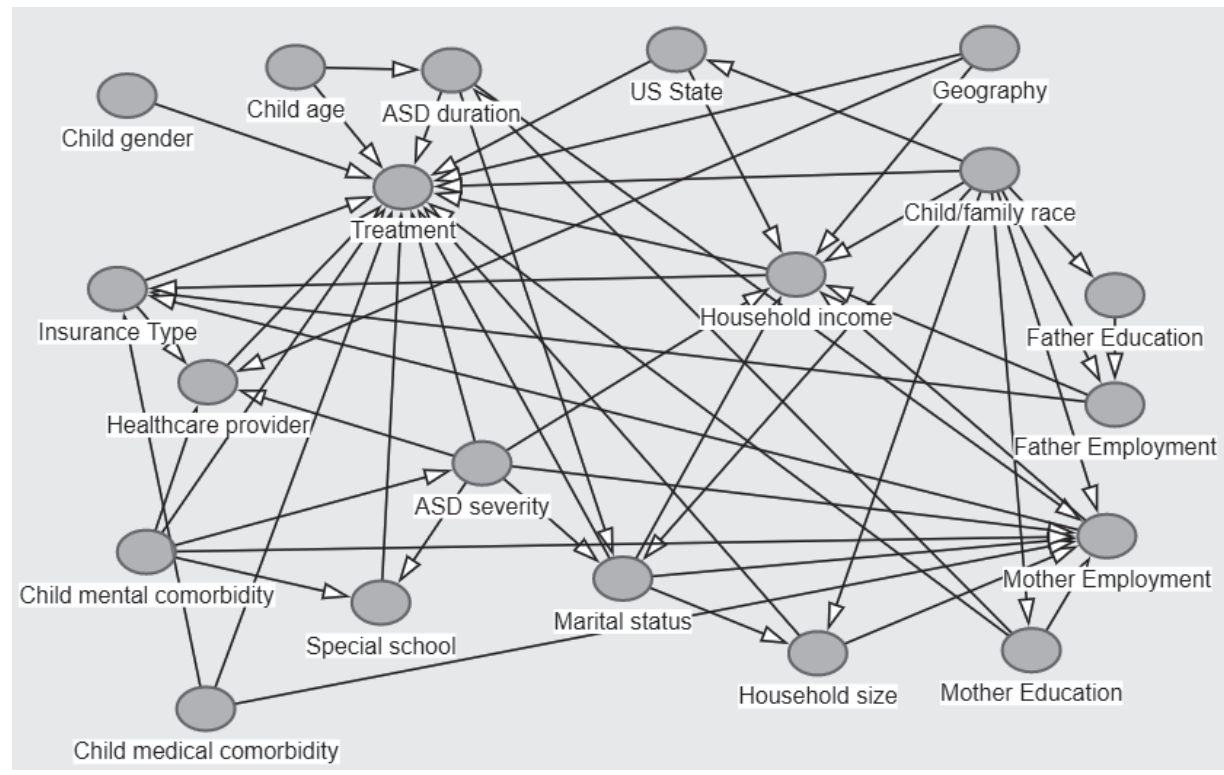

ASD: autism spectrum disorder 


\section{S.2.3.2: Participant flow}

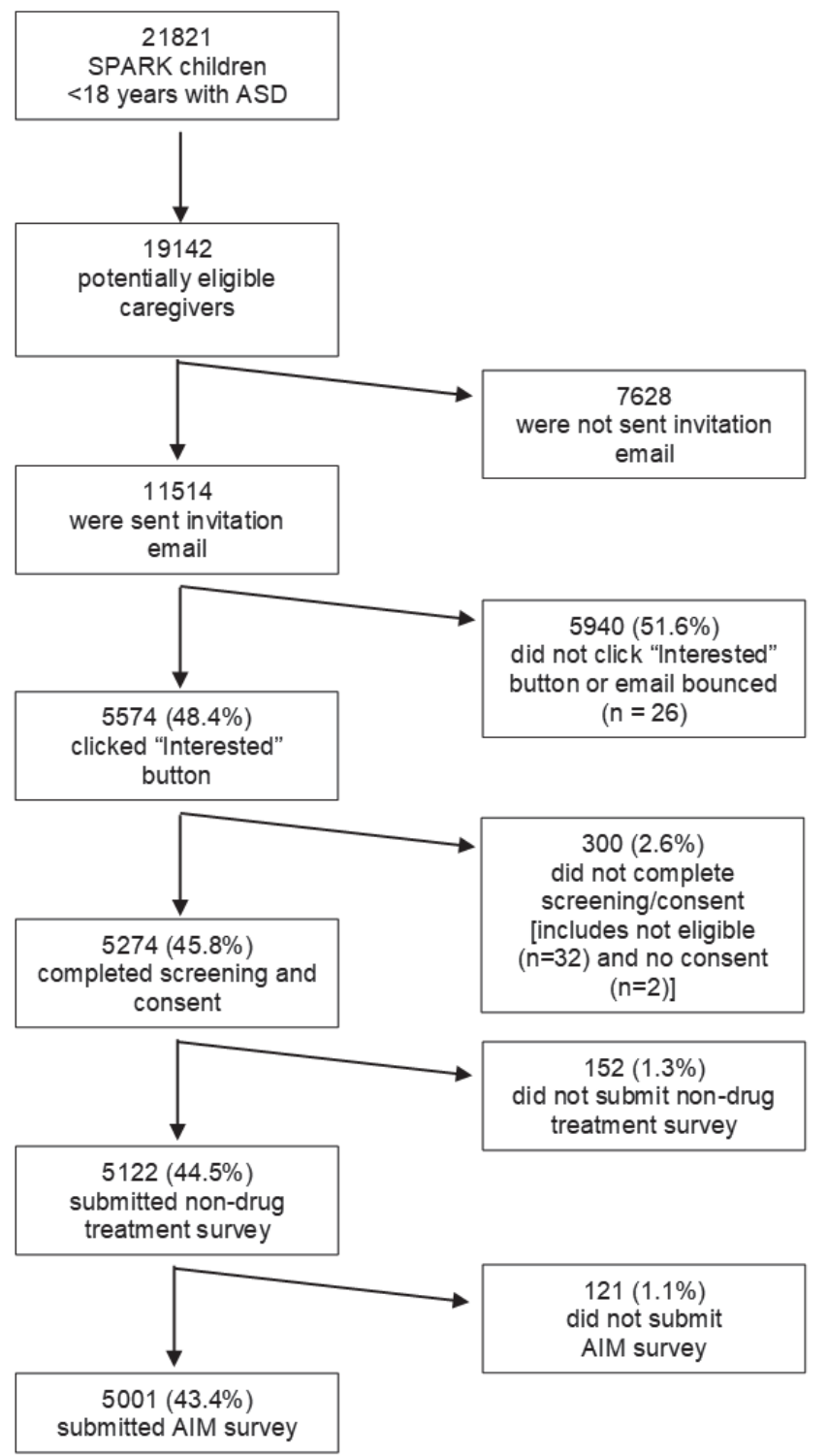

Parentheses show \% of total participants sent the invitation email 


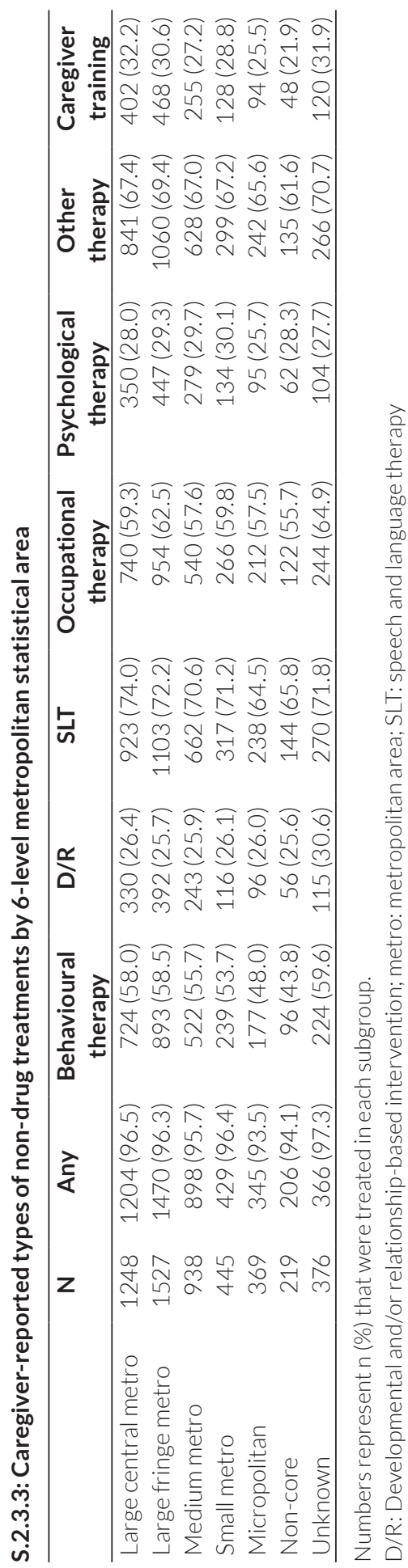




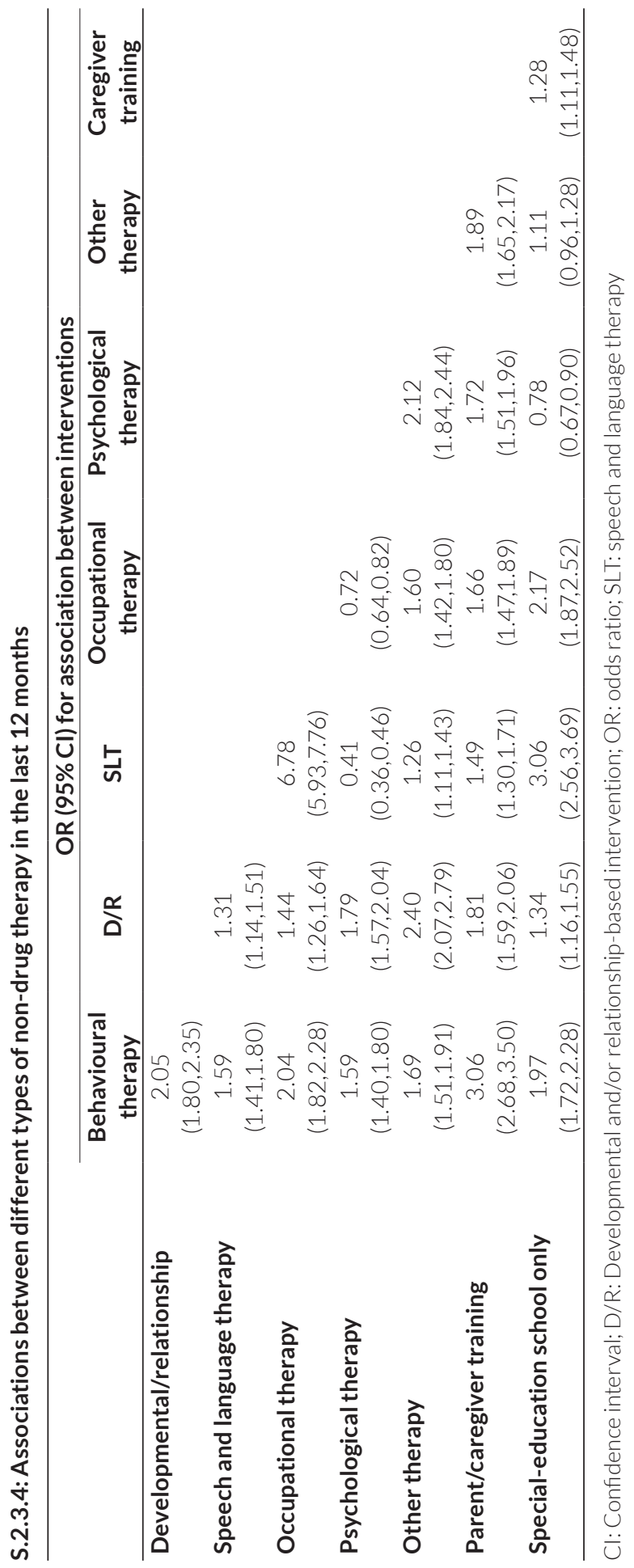


S.2.3.5: Patterns of treatments of non-drug therapy for all groups with $>2 \%$

\begin{tabular}{|c|c|c|c|c|c|c|c|c|}
\hline $\begin{array}{c}\text { Behavioural } \\
\text { therapy }\end{array}$ & $D / R$ & SLT & $\begin{array}{c}\text { Occupational } \\
\text { therapy }\end{array}$ & $\begin{array}{c}\text { Psychological } \\
\text { therapy }\end{array}$ & $\begin{array}{c}\text { Other } \\
\text { therapy }\end{array}$ & $\begin{array}{c}\text { Caregiver } \\
\text { training }\end{array}$ & $\mathrm{n}$ & $\%$ \\
\hline \multicolumn{9}{|c|}{ Overall $(n=5,122)$} \\
\hline Y & $\mathrm{N}$ & Y & Y & N & Y & N & 362 & 7.1 \\
\hline$N$ & $\mathrm{~N}$ & Y & Y & $N$ & Y & $\mathrm{N}$ & 302 & 5.9 \\
\hline N & $\mathrm{N}$ & Y & Y & $\mathrm{N}$ & N & $\mathrm{N}$ & 243 & 4.7 \\
\hline Y & N & Y & Y & N & Y & Y & 235 & 4.6 \\
\hline N & $\mathrm{N}$ & $N$ & $N$ & $N$ & N & $\mathrm{N}$ & 185 & 3.6 \\
\hline Y & $\mathrm{N}$ & Y & Y & $\mathrm{N}$ & N & $\mathrm{N}$ & 180 & 3.5 \\
\hline N & $\mathrm{N}$ & Y & N & N & Y & $\mathrm{N}$ & 153 & 3.0 \\
\hline Y & Y & Y & Y & N & Y & $\mathrm{N}$ & 150 & 2.9 \\
\hline N & $\mathrm{N}$ & Y & $\mathrm{N}$ & N & N & $\mathrm{N}$ & 146 & 2.9 \\
\hline Y & Y & Y & Y & N & Y & Y & 143 & 2.8 \\
\hline N & N & N & $\mathrm{N}$ & $\mathrm{N}$ & Y & $\mathrm{N}$ & 140 & 2.7 \\
\hline \multicolumn{9}{|c|}{ By geography: Metropolitan $(n=588)$} \\
\hline Y & N & Y & Y & N & Y & N & 305 & 7.3 \\
\hline N & $\mathrm{N}$ & Y & Y & N & Y & N & 225 & 5.4 \\
\hline Y & $\mathrm{N}$ & Y & Y & N & Y & Y & 202 & 4.9 \\
\hline N & $\mathrm{N}$ & Y & Y & N & N & N & 186 & 4.5 \\
\hline Y & $\mathrm{N}$ & Y & Y & N & N & $\mathrm{N}$ & 152 & 3.7 \\
\hline N & $\mathrm{N}$ & $N$ & $N$ & $N$ & $N$ & $\mathrm{~N}$ & 142 & 3.4 \\
\hline $\mathrm{N}$ & $N$ & $Y$ & $\mathrm{~N}$ & $N$ & $Y$ & $N$ & 126 & 3.0 \\
\hline$Y$ & $Y$ & $Y$ & $Y$ & $N$ & $Y$ & $\mathrm{~N}$ & 124 & 3.0 \\
\hline$Y$ & $Y$ & $Y$ & $Y$ & $N$ & $Y$ & $Y$ & 121 & 2.9 \\
\hline$N$ & $\mathrm{~N}$ & $Y$ & $N$ & $N$ & $N$ & $\mathrm{~N}$ & 112 & 2.7 \\
\hline$N$ & $\mathrm{~N}$ & $N$ & $N$ & $N$ & $Y$ & $\mathrm{~N}$ & 111 & 2.7 \\
\hline$Y$ & $\mathrm{~N}$ & $Y$ & $Y$ & $Y$ & $Y$ & $Y$ & 87 & 2.1 \\
\hline$Y$ & $\mathrm{~N}$ & $Y$ & $Y$ & $Y$ & $Y$ & $\mathrm{~N}$ & 86 & 2.1 \\
\hline \multicolumn{9}{|c|}{ By geography: Nonmetropolitan $(n=4,158)$} \\
\hline N & $\mathrm{N}$ & Y & Y & $\mathrm{N}$ & Y & $\mathrm{N}$ & 47 & 8.0 \\
\hline N & N & Y & Y & N & N & N & 41 & 7.0 \\
\hline N & N & N & $\mathrm{N}$ & N & N & $\mathrm{N}$ & 35 & 6.0 \\
\hline Y & N & Y & Y & N & Y & N & 31 & 5.3 \\
\hline N & N & Y & $\mathrm{N}$ & N & N & N & 28 & 4.8 \\
\hline N & N & $N$ & $\mathrm{~N}$ & $N$ & $Y$ & $\mathrm{~N}$ & 23 & 3.9 \\
\hline$N$ & $\mathrm{~N}$ & $Y$ & $\mathrm{~N}$ & $\mathrm{~N}$ & $Y$ & $\mathrm{~N}$ & 19 & 3.2 \\
\hline$Y$ & $\mathrm{~N}$ & $Y$ & $Y$ & $N$ & $N$ & $\mathrm{~N}$ & 16 & 2.7 \\
\hline$Y$ & $\mathrm{~N}$ & $Y$ & $\mathrm{~N}$ & $N$ & $Y$ & $\mathrm{~N}$ & 14 & 2.4 \\
\hline$Y$ & $\mathrm{~N}$ & $Y$ & $Y$ & Y & $Y$ & $\mathrm{~N}$ & 13 & 2.2 \\
\hline
\end{tabular}

D/R: Developmental and/or relationship-based intervention; SLT: speech and language therapy; N: No; Y: Yes 


\section{S.2.3.6: Intensity of treatment (hours/week) by metropolitan statistical area (MSA)}

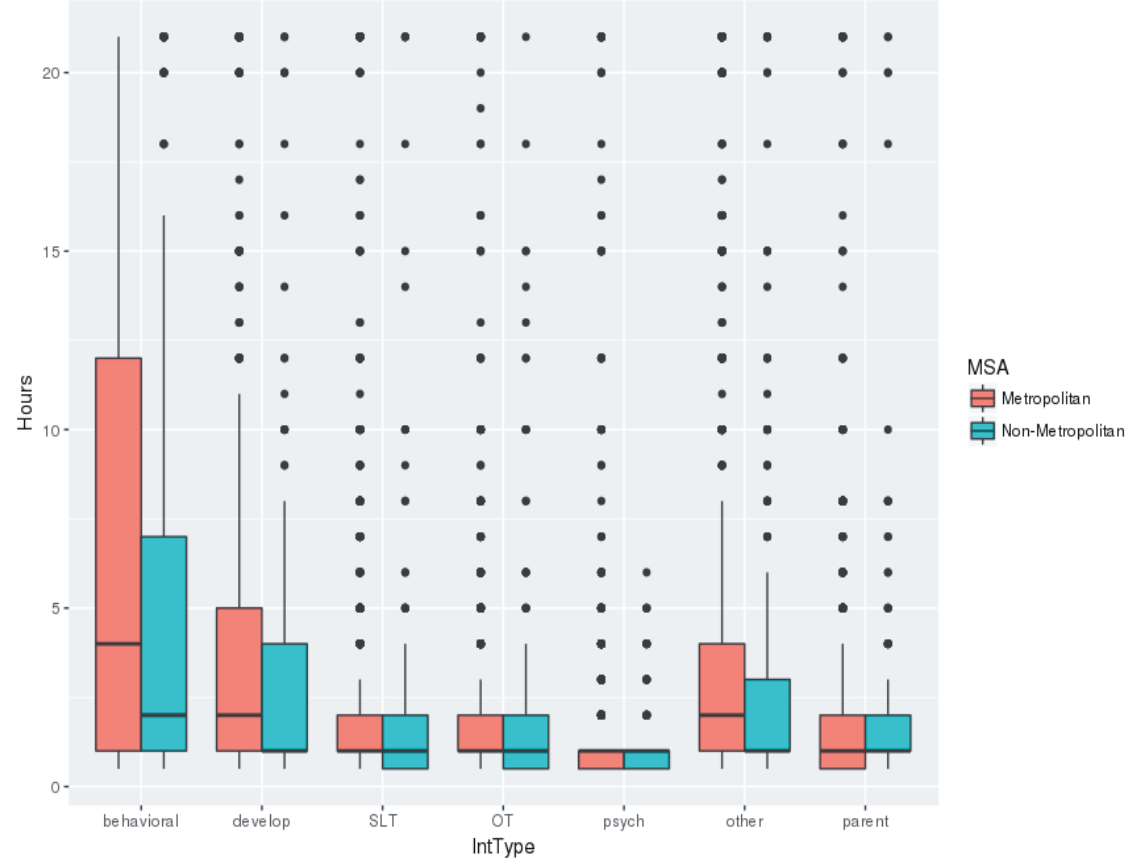

Behavioral: Behavioural intervention; Develop: Developmental and/or relationship-based intervention; IntType: Intervention Type; MSA: Metropolitan Statistical Area; OT: Occupational Therapy; Other: Other intervention; Parent: Parent/Caregiver training; Psych: Psychological intervention; SLT: Speech and language therapy 


\section{S.2.3.7: Intensity of treatment (hours/week) by insurance type}

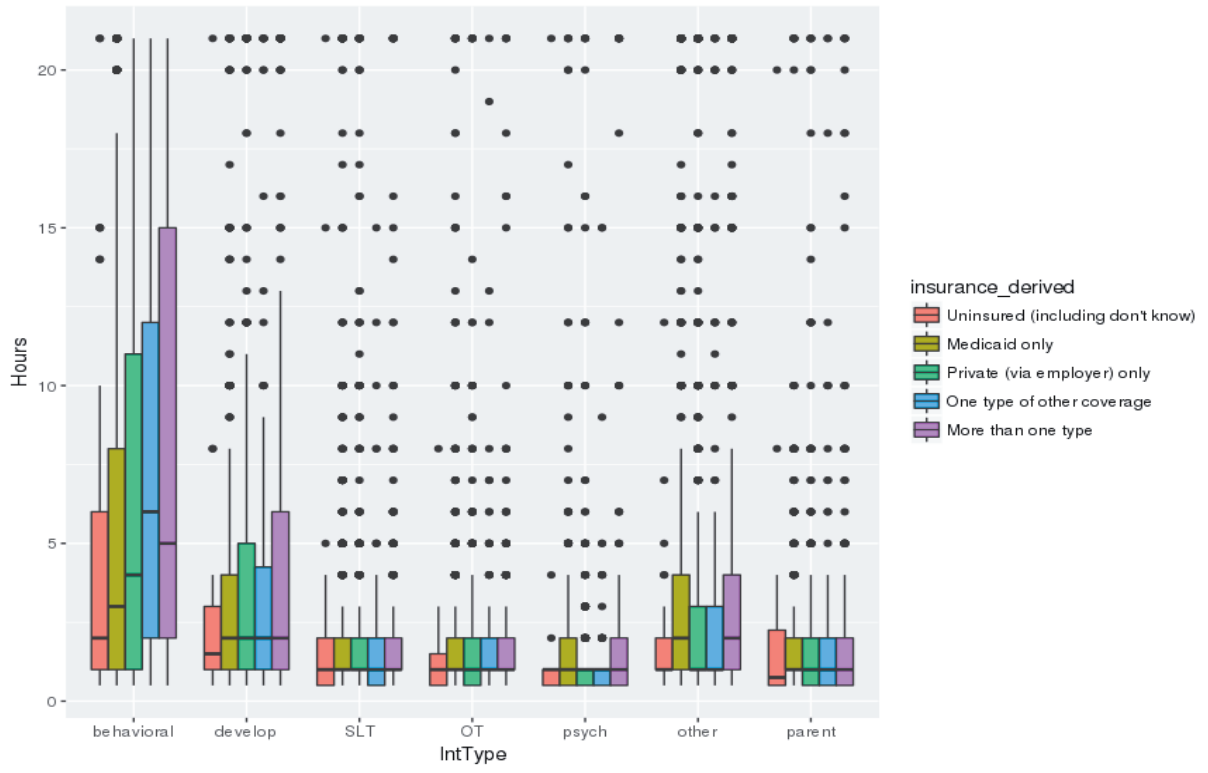

Behavioral: Behavioural intervention; Develop: Developmental and/or relationship-based intervention; IntType: Intervention Type; OT: Occupational Therapy; Other: Other intervention; Parent: Parent/ Caregiver training; Psych: Psychological intervention; SLT: Speech and language therapy 


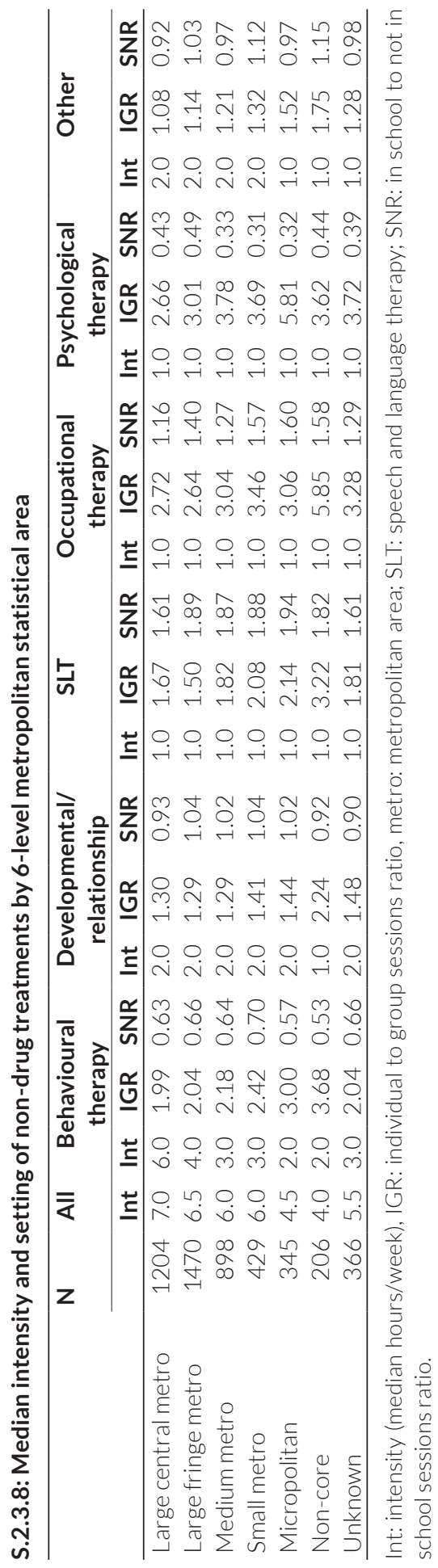


S.2.3.9: Association of caregiver-reported type and intensity of non-drug therapy with insurance (PS trimmed analyses)

\begin{tabular}{|c|c|c|}
\hline \multirow[b]{2}{*}{ Non-drug therapy type } & \multicolumn{2}{|c|}{$\begin{array}{l}\text { Insurance } \\
\text { (Only private via employer vs only Medicaid) } \\
\mathrm{N}=1,625^{*}\end{array}$} \\
\hline & PS-adjusted & Double-adjusted \\
\hline & \multicolumn{2}{|c|}{ Association with type of therapy OR $(95 \% \mathrm{Cl})$} \\
\hline Any & $0.57(0.31,1.04)$ & $0.53(0.28,0.99)$ \\
\hline Behavioural & $0.84(0.64,1.10)$ & $0.84(0.63,1.13)$ \\
\hline Developmental/ relationship & $1.27(0.94,1.72)$ & $1.32(0.95,1.83)$ \\
\hline SLT & $0.91(0.68,1.21)$ & $0.90(0.64,1.28)$ \\
\hline Occupational & $0.86(0.66,1.14)$ & $0.85(0.62,1.16)$ \\
\hline Psychological & $0.80(0.61,1.07)$ & $0.77(0.55,1.06)$ \\
\hline \multirow[t]{2}{*}{ Other } & $0.89(0.67,1.18)$ & $0.85(0.62,1.17)$ \\
\hline & \multicolumn{2}{|c|}{ Association with intensity of therapy $\mathrm{RR}(95 \% \mathrm{Cl}$} \\
\hline Any & $0.94(0.80,1.11)$ & $0.89(0.77,1.03)$ \\
\hline Behavioural & $1.04(0.84,1.28)$ & $0.98(0.78,1.22)$ \\
\hline Developmental/relationship & $1.49(1.03,2.17)$ & $1.21(0.86,1.71)$ \\
\hline SLT & $0.94(0.74,1.21)$ & $0.90(0.76,1.08)$ \\
\hline Occupational & $0.86(0.68,1.08)$ & $0.89(0.72,1.10)$ \\
\hline Psychological & $0.95(0.66,1.38)$ & $0.92(0.63,1.33)$ \\
\hline Other & $0.71(0.58,0.86)$ & $0.69(0.56,0.85)$ \\
\hline
\end{tabular}

CI: Confidence interval; OR: Odds Ratio; PS: propensity score; RR: Rate ratio; SLT: Speech and language therapy

Models were adjusted for below variables using propensity score inverse probability weighting (adjusted and double adjusted: see methods section)

Variables for adjustment: AIM domain scores (continuous), child race/ethnicity (White/Hispanic, White/ Non-Hispanic, Non-White/Hispanic, Non-White/Non-Hispanic), child other medical problems (yes/no), child other mental health or psychiatric problems (yes/no), geography (nonmetropolitan/ metropolitan), household income (four strata, $\geq \$ 20,000$ to $\leq \$ 99,999$ ), marital status (married/living with partner yes/ no), mother employment (work full time/part time yes/no; excluded fathers), US state (excluded states with $n<3$ for Medicaid and private employer insurance).

* 7 participants trimmed ( 2 from only Medicaid group, 5 from only private via employer group) 


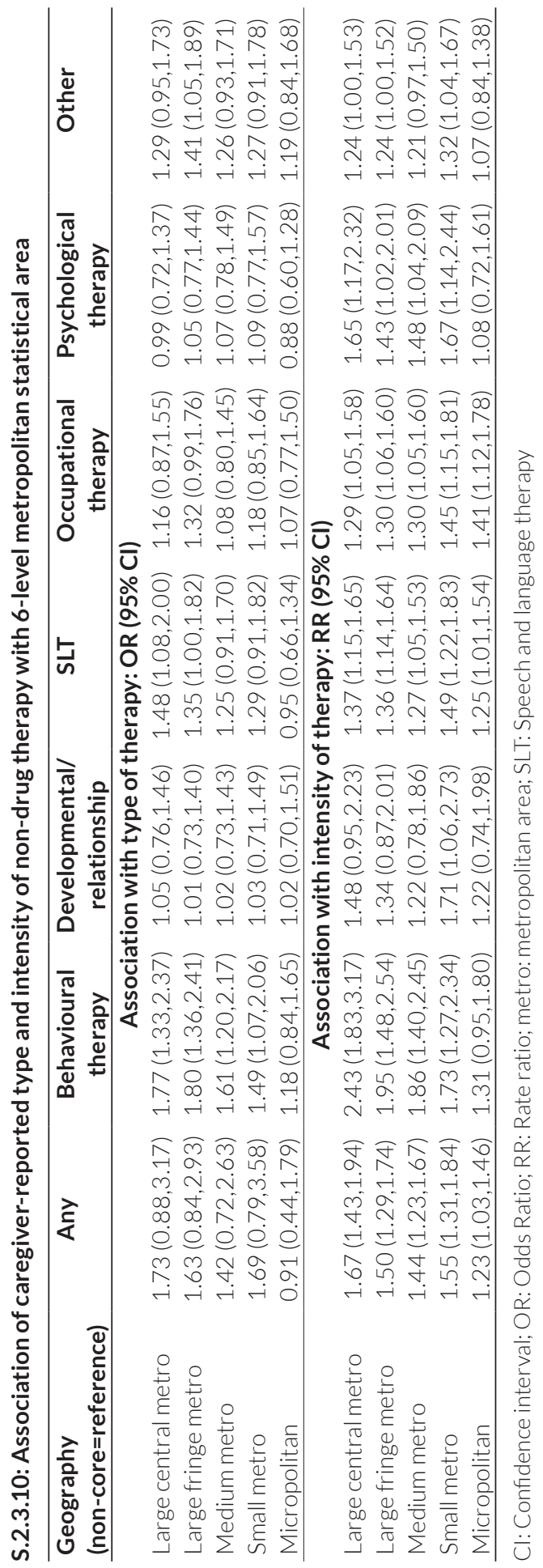




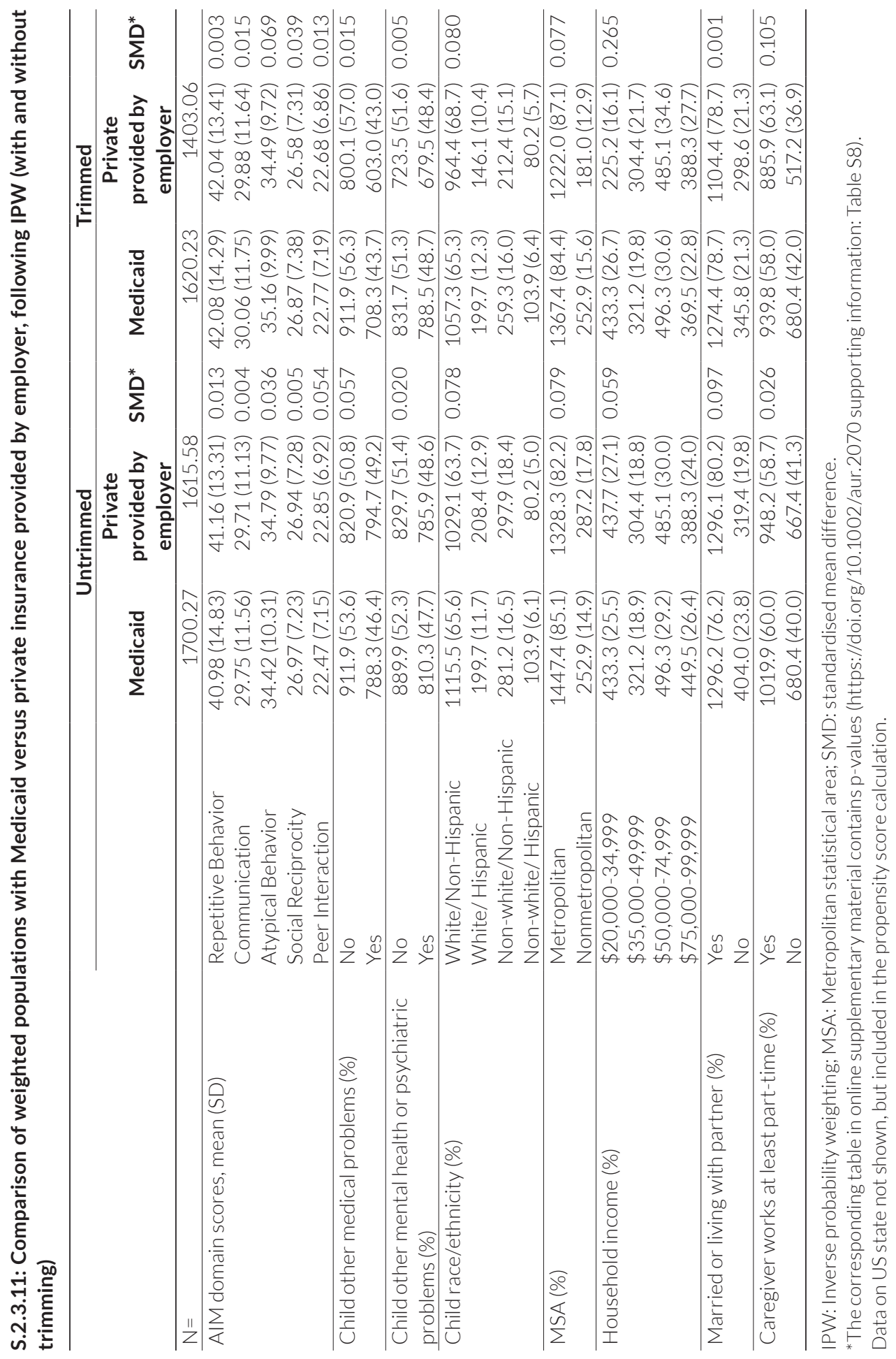


Treatment patterns in children with autism in the United States 



\section{Chapter 3}

Safety evaluation of treatments in children with autism spectrum disorder 


\section{Chapter 3.1}

Psychostimulants/atomoxetine and serious cardiovascular events in children with ADHD or autism spectrum disorder
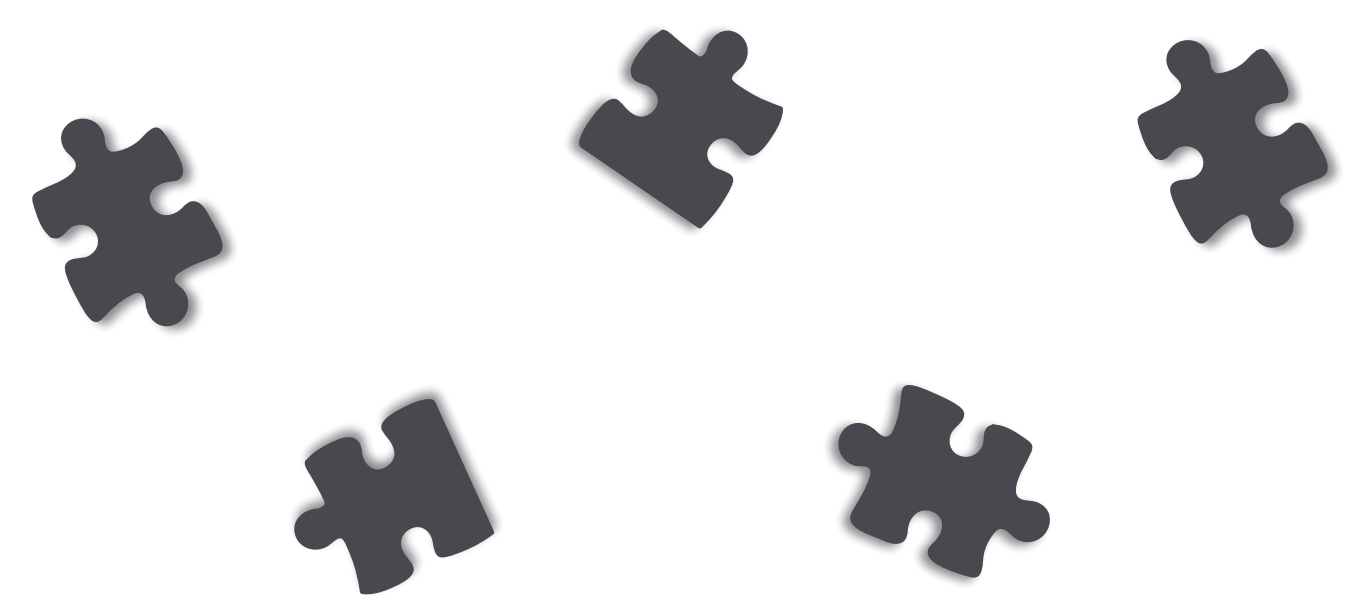


\section{Abstract}

Psychostimulants and atomoxetine have been shown to increase blood pressure, heart rate, and QT interval in children and adolescents; however, based on current literature, it is unclear if these "attention-deficit/hyperactivity disorder (ADHD) medications" are also associated with serious cardiovascular (SCV) events. We addressed this question in commonly exposed groups of children and adolescents with either ADHD or autism spectrum disorder (ASD). Using commercial (years 2000-2016) and Medicaid (years 2012-2016) administrative claims data from the United States (US), we conducted two case-control studies, nested within respective cohorts of ADHD and ASD children aged 3-18 years. We defined cases by a composite outcome of stroke, myocardial infarction, or serious cardiac arrhythmia. For each case, we matched ten controls on age, sex, and insurance type. We conducted conditional logistic regression models to test associations between SCV outcomes and a primary exposure definition of current ADHD medication use. Additionally, we controlled for resource use, cardiovascular and psychiatric comorbidities, and use of medications in a variety of sensitivity analyses. We identified 2,240,774 children for the ADHD cohort and 326,221 children for the ASD cohort. For ADHD, 33.9\% of cases (63 of 186) versus 32.2\% of controls (598 of 1860) were exposed, which yielded an odds ratio and 95\% confidence interval (OR) of 1.08 (0.78-1.49). For ASD, 12.5\% of cases (6 of 48) versus $22.1 \%$ of controls (106 of 480) were exposed [OR 0.49 (0.20-1.20)]. Covariate-adjusted results and results for individual outcomes and other exposure definitions were consistent with no increased risk of SCV events. Using large US claims data, we found no evidence of increased SCV risk in children and adolescents with ADHD or ASD exposed to ADHD medications.

\section{Lay summary}

ADHD medications have been shown to increase blood pressure and heart rate in children. Using historical insurance claims data, we tested if these drugs were also related with increased risks of serious cardiovascular events like stroke or heart attack, in children with ADHD and ASD. We found this not to be the case. There were no significant differences in ADHD medication use between patients who did or did not have a serious event. 


\section{Introduction}

Prescription stimulants such as methylphenidate and non-stimulants such as atomoxetine are labelled for the treatment of attention-deficit/hyperactivity disorder (ADHD) in from age approximately 6 and above years, and are also commonly used in medical practice for the management of non-core symptoms of autism spectrum disorder (ASD) ${ }^{1}$. In the US and in Europe, there has been a trend toward higher prescription rates of these drugs (hereon referred to as "ADHD medications") for both children and adults over the past two decades, nevertheless, use remains most common in middle childhood and not all ADHD diagnosed individuals are prescribed ADHD medications ${ }^{2-7}$.

Despite evidence for short-term effectiveness ${ }^{8}$, both placebo-controlled and open-label extension trials have repeatedly shown ADHD medication-induced increases in mean blood pressure, heart rate, and QT interval in children, adolescents, and adults ${ }^{9-11}$. Although these increases were described as relatively minor, their existence has raised concern to what degree ADHD medication could influence the likelihood of serious cardiovascular (SCV) events such as stroke, myocardial infarction (MI), and cardiac arrhythmia, especially in people with underlying heart problems ${ }^{11}$. Furthermore, ADHD medications have been linked to sudden cardiac death in case reports and currently carry a US Food and Drug Administration (FDA) class-specific warning regarding these potential increased risks ${ }^{12-14}$.

A limited number of observational studies have generally found no increased risk of SCV events with ADHD medication use, but results have not been consistent ${ }^{14}$. The majority of such studies were conducted on data from over a decade ago and due to the low absolute numbers of SCV events, the ability to rule out such an association has been limited ${ }^{15,16}$. To the best of our knowledge, no studies have specifically studied this question in the growing subgroup of exposed children with ASD, who frequently use other psychotropic co-medications such as antidepressants or antipsychotics ${ }^{17}$, which may further increase heart rate, QT interval, and consequently the risk of SCV events.

Given the uncertainty described above, which surrounds the relationship between SCV events and ADHD medications, plus the increasing number of children and adolescences with ADHD and ASD that are exposed, our study aimed to quantify this risk, in large cohorts, representative of these populations.

\section{Methods}

\section{Study design and data}

This was a retrospective, nested case-control study using the Truven Health MarketScan administrative insurance claims database. At the time of analysis, the full database contained billed records of care on 184 million commercially insured and 19 million 
Medicaid insured individuals between calendar years 2000-2016 (with at least some coverage from each US state) and 2012-2016 (10-12 states), respectively.

\section{Cohort selection and follow-up}

From within the whole MarketScan database, we defined two main cohorts of interest: individuals with (a) ADHD (but not ASD) and (b) individuals with ASD (with or without ADHD). Eligibility requirements were: two or more claims for ASD or ADHD respectively; age between 3 and 18 years; and individuals were excluded from the ASD cohort if they ever had any claim for Rett's syndrome, to avoid possibly misdiagnosed cases ${ }^{17-19}$. To avoid overlap between the two cohorts, individuals with ASD claims were removed from the ADHD cohort, but not vice versa. This decision was made because previous studies have shown over 1 in 3 autistic people have an ADHD comorbidity versus a lower proportion (around 1 in 8) of the ADHD population with comorbid ASD ${ }^{20,21}$. We also excluded individuals with any previous SCV event of interest prior to diagnosis and start of follow up. Individuals in both cohorts were followed from first ASD diagnosis claim (minimum age of 3 years) until first SCV event, the end of database enrolment, or end of calendar year marking their 18th birthday, whichever occurred first.

\section{Outcomes and case/control selection}

From within each of the two cohorts, we conducted a nested case-control study. Cases were identified by the first inpatient primary diagnosis claim for any of the three secondary SCV outcomes, namely: (1) stroke, (2) MI, and (3) serious cardiac arrhythmia (SCA). SCA included cardiac arrest, complete atrioventricular block, and ventricular tachycardia, ventricular fibrillations or flutter. Definitions were based upon previously published studies and systematic reviews which show high positive predictive values (PPV >85\%) ${ }^{15,22-27}$. For each case we defined the index date as the date of the composite (first) event.

For each case, 10 controls were matched, randomly and without replacement, using the risk set sampling technique ${ }^{28}$. Matching was based on age, sex, insurance type, and calendar time, so controls were assigned the same index date as their case. Finally, both cases and controls were required to have at least 30-days continuous enrolment in the database, directly prior to the index date. This was needed in order to establish baseline risk factors and to observe exposures.

\section{Exposure definitions}

Based on dispense date and days-supply, the primary exposure variable was defined as currently versus not-currently exposed to any ADHD medication on the index date. As per previous studies ${ }^{15,23}$, current use was deemed to be the most etiologically relevant exposure as the half-life of stimulants/atomoxetine is short (hours opposed to days).

\section{Statistical analysis}

After matching, we used conditional logistic regression to perform the crude (matched) analysis. Beyond the crude analysis, a causal diagram was used to identify other covariates 
to be included in a minimal adjustment set (see supplement S.3.1.1 and Greenland et al ${ }^{29}$ and Textor et al ${ }^{30}$ for diagram theory). We refer to this set as adjustment set 1 , which included the concepts of underlying cardiovascular risk and healthcare resource use (HCRU). We defined underlying cardiovascular risk by taking prior record of the following comorbidities into account: congenital circulatory system disorders, congestive heart failure (CHF), essential hypertension, disorders of lipid metabolism, peripheral artery disease, asthma, chronic obstructive pulmonary disease (COPD), diabetes, and obesity ${ }^{15,23}$. We approximated HCRU via presence/absence of a visit to the emergency department, cardiology specialist, behavioural therapist, inpatient visit for any reason, and the total number of medical claims pro-rated to the past year. When deriving these variables, we ignored data during the month prior to index, in order to avoid overadjustment bias by using data potentially collected post exposure. The total number of medical claims in the past year was pro-rated for individuals with less than 12 months prior follow up. Covariate adjustment was made by selecting a weighted subset of the controls that had characteristics most similar to the cases. To achieve optimal covariate balance between cases and controls, weights were assigned by a generalised boosted model algorithm ${ }^{31}$, before unmatched logistic regression was applied to test the exposureoutcome association.

\section{Sensitivity analyses}

We conducted two sets of sensitivity analyses and a post-hoc subgroup analysis. In the first sensitivity analysis, we adjusted exposure definitions to within 90-days and "ever use" prior to index. In the second sensitivity analysis, we additionally controlled for an expanded set of other covariates. Adjustment set 2 included all covariates from adjustment set 1 as well as severe medical comorbidities, common psychiatric comorbidities, psychotropic medications, and beta-blocker use. Psychotropic and beta-blocker medication use were based on prescriptions in the 6-months prior to index. These covariates were selected a priori using potential confounders and clinical assumptions from the literature (but not using causal diagrams like adjustment set 1). Additionally, we adjusted for both adjustment sets via adjusted conditional logistic regression to test if model specification had an impact on findings.

Lastly, in a post-hoc subgroup analysis, we excluded cases and controls with either congenital circulatory system disorders, congestive heart failure, or any cardiology specialty visit in the past year (and their matched pairs). We also repeated crude and weighted analysis by individual endpoints (stroke, MI, SCA). Throughout, results were deemed statistically significant or not based upon 95\% confidence intervals. 


\section{Results}

A total of 2,240,774 children and adolescents were identified for the ADHD cohort and 326,221 were identified for the ASD cohort (Table 3.1.1). The ADHD cohort had 1,531,687 (68.4\%) males and the mean (standard deviation; SD) age at first ADHD claim was 11.1 (3.7) years. This cohort had 186 composite SCV events over a mean (SD) 2.66 (2.11) years of at-risk time, resulting in an incidence rate (95\% confidence interval; CI) of 3.12 (2.70-3.60) per 100,000 person years. The ASD cohort had 262,434 (80.4\%) males and the mean (SD) age at first ASD claim was 9.3 (4.4) years. This cohort had 48 composite SCV events over a mean (SD) 2.62 (2.14) years of at-risk time, resulting in an incidence rate $(95 \% \mathrm{Cl}$ ) of $5.62(4.23-7.45)$ per 100,000 person years. The most common specific event in both cohorts was stroke, and MI was the rarest. See supplements S.3.1.2 and S.3.1.3 for full listings of events.

\section{Table 3.1.1: Attrition table and selection of cohorts}

\begin{tabular}{lrr}
\hline & ADHD & ASD \\
\hline At least 1 claim for ASD/ADHD at age at least 3 years & $5,978,601$ & 612,856 \\
At least 2 claims for ASD/ADHD at age at least 3 years & $4,428,572$ & 452,851 \\
Exclude individuals with claim for ASD (from ADHD & $4,211,082$ & 451,832 \\
cohort) and Rett's syndrome (from ASD cohort) & & \\
Only include individuals enrolled for some time & $2,240,854$ & 326,246 \\
between ages 3-18 (inclusive) and first claim for ASD/ & & \\
ADHD at age before 19 years & & \\
Exclude individuals with event of interest (stroke, & $2,240,774$ & 326,221 \\
myocardial infarction, serious cardia arrhythmia) prior & & 186 \\
to first ASD/ADHD diagnosis claim & 102 & 22 \\
Total SCV events & 10 & 75 \\
$\quad$ Stroke & 75 \\
$\quad$ Myocardial infarction & & \\
Serious cardiac arrhythmia & & \\
\hline
\end{tabular}

Composite event was the main event of interest, defined as the first of individual events.

ADHD = attention-deficit/hyperactivity disorder; ASD = autism spectrum disorder; SCV events = serious cardiovascular events.

Characteristics of cases and controls, selected from the ADHD and ASD cohorts based on the composite SCV endpoint, are presented in Table 3.1.2. We found 10 controls for each case as planned on the matching characteristics (age, sex, and insurance). Cases in both cohorts more often had underlying cardiovascular comorbidities and higher amounts of inpatient, emergency and cardiology resource use than controls. ADHD cases were on average slightly older at time of SCV event compared to ASD cases (mean 13.9 vs 12.5 years) and received fewer psychotropic drugs. By design, none of the ADHD cases has comorbid ASD, but 9 (18\%) of the ASD cases had comorbid ADHD. 
Table 3.1.2: ADHD and ASD cases and control characteristics (based on composite serious cardiovascular event)

\begin{tabular}{|c|c|c|c|c|c|c|}
\hline & \multicolumn{3}{|c|}{ ADHD } & \multicolumn{3}{|c|}{ ASD } \\
\hline & $\begin{array}{l}\text { Cases } \\
\mathrm{N}=186\end{array}$ & $\begin{array}{l}\text { Controls } \\
N=1,860\end{array}$ & SMD & $\begin{array}{l}\text { Cases } \\
\mathrm{N}=48\end{array}$ & $\begin{array}{l}\text { Controls } \\
\mathrm{N}=480\end{array}$ & SMD \\
\hline \multicolumn{7}{|c|}{ Demographics (initial matching criteria) } \\
\hline & $53(28.5)$ & $530(28.5)$ & NA & $10(20.8)$ & $100(20.8)$ & \\
\hline Age in $y$ & $13.9(3.4)$ & $13.9(3.4)$ & & $12.5(4.4)$ & $12.5(4.4)$ & \\
\hline ars & & & & & & \\
\hline $3-4$ & $0(0.0)$ & $0(0.0)$ & & $4(8.3)$ & $40(8.3)$ & \\
\hline $5-9$ & $20(10.8)$ & $200(10.8)$ & & $9(18.8)$ & $90(18.8)$ & \\
\hline-14 & $78(41.9)$ & $780(41.9)$ & & $15(31.2)$ & $150(31.2)$ & \\
\hline & $88(47.3)$ & $880(47.3)$ & & $20(41.7)$ & $200(41.7)$ & \\
\hline Medi & $58(31.2)$ & $580(31.2)$ & & $14(29.2)$ & $140(29.2)$ & \\
\hline Capitated ins & $52(28.0)$ & $520(28.0)$ & & $14(29.2)$ & $140(29.2)$ & \\
\hline \multicolumn{7}{|c|}{ History of cardiovascular comorbidities } \\
\hline Congenital circulator & $29(15.6)$ & $20(1.1)$ & 0.544 & $9(18.8)$ & $11(2.3)$ & 0.557 \\
\hline Congestive hea & $11(5.9)$ & $1(0.1)$ & & $6(12.5)$ & $0(0.0)$ & 0.535 \\
\hline & $11(5.9)$ & $32(1.7)$ & & $2(4.2)$ & $14(2.9)$ & 0.068 \\
\hline Disorde & $5(2.7)$ & $30(1.6)$ & 0.074 & $2(4.2)$ & $9(1.9)$ & 0.134 \\
\hline se & $3(1.6)$ & $1(0.1)$ & 0.1 & $1(2.1)$ & $0(0.0)$ & 0.206 \\
\hline & $38(20.4)$ & $295(15.9)$ & 0.1 & $9(18.8)$ & 85 ( 17.7) & 0.027 \\
\hline Chronic obstr & $1(0.5)$ & $5(0.3)$ & 0.042 & $1(2.1)$ & $7(1.5)$ & 0.047 \\
\hline e & & & & & & \\
\hline es & & & & & & 64 \\
\hline & & & & & & \\
\hline \multicolumn{7}{|l|}{ HCRU } \\
\hline & 71( & $350(18.8)$ & 0.4 & 21( & 9.4) & 0.543 \\
\hline & $32(17.2)$ & $51(2.7)$ & 0.4 & 5) & $.8)$ & 0.324 \\
\hline & $35(18.8)$ & $33(1.8)$ & 0.5 & $9(18.8)$ & 4) & 0.461 \\
\hline apy & 57( & $452(24.3)$ & 0.142 & $7(14.6)$ & 1631 & 0.464 \\
\hline Days with any me & 21.5 & 12.0 & 0.349 & $28.6(3$ & 22.7 & 0.160 \\
\hline \multicolumn{7}{|l|}{ Psychiatric comorbidities } \\
\hline & NA & NA & NA & $.8)$ & 191 & 0.475 \\
\hline & & & & 8) & 107 & 0.03 \\
\hline n & .7) & $215(1$ & & 5 & 54 & 0.02 \\
\hline & 7.0) & $32(1.7)$ & 0.2 & $9(1$ & 41 & 0.30 \\
\hline & & & & & & 0 \\
\hline \multicolumn{7}{|l|}{ Other serious medical conditions } \\
\hline Cancer & $12(6.5)$ & $7(0.4)$ & 0 . & & & 0.065 \\
\hline & & $0.2)$ & 0. & & & 0.06 \\
\hline & $4(2.2)$ & $6(0.3)$ & 0.1 & & & 0.10 \\
\hline Human immunodeficiency & & & & & & 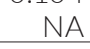 \\
\hline \multicolumn{7}{|l|}{ Psychotropic medications } \\
\hline & $33(17.7)$ & $222(11.9)$ & & $8(16.7)$ & $123(25.6)$ & 0.221 \\
\hline & $7(3.8)$ & $110(5.9)$ & & $13(27.1)$ & $98(20.4)$ & 0.15 \\
\hline ytics/Sedatives/Hypnotics & $4(2.2)$ & $38(2.0)$ & 0.008 & $6(12.5)$ & $14(2.9)$ & 0.365 \\
\hline & $13(7.0)$ & $16(0.9)$ & 0.3 & $8(16.7)$ & $21(4.4)$ & 0.409 \\
\hline eta-blockers & $12(6.5)$ & $3(0.2)$ & 0.357 & $2(4.2)$ & $2(0.4)$ & 0.253 \\
\hline
\end{tabular}

Results are $(\%)$ unless stated otherwise. $A D H D=$ attention-deficit/hyperactivity disorder; $A S D=$ autism spectrum disorder; HCRU = healthcare resource use; NA = not applicable; $\mathrm{SD}$ = standard deviation; $\mathrm{SMD}=$ standardised mean difference between cases and controls 
Table 3.1.3 shows that for both ADHD and ASD, there was no increased risk of SCV events associated with ADHD medication use. For ADHD, the proportion of cases currently exposed was $33.9 \%$ (63 of 186 cases) versus $32.2 \%$ of controls (598 of 1,860 controls). This translated to no association of ADHD medication use with SCV events in the crude analysis [odds ratio (OR) $(95 \% \mathrm{Cl}) 1.08(0.78-1.49)]$. For ASD, the proportion of cases currently exposed was $12.5 \%$ (6 of 48 cases) versus $22.1 \%$ of controls (106 of 480 controls). This also translated to no crude association of ADHD medication use with SCV events in the ASD cohort [OR (95\% Cl) 0.49 (0.20-1.20)].

Furthermore, based on the current exposure definition, and across both ADHD and ASD cohorts, all results statistically adjusted for covariates were consistent with these findings (Table 3.1.3). For weighted cohort characteristics, see supplements S.3.1.4 and S.3.1.5. After completely excluding individuals with underlying congenital circulatory system disorders, CHF, or recent cardiology visits, odds ratios were closer to a null association than in crude (and most adjusted) analyses.

Point estimates for associations between the outcomes and exposures were also stable (and without trend) regardless of the exposure definition used. Due to small sample sizes, some of the associated confidence intervals were wide, especially in the ASD cohort. There were no obvious differences in specific drugs or dosages used, between cases and controls nor ASD and ADHD (see supplement S.3.1.6). Finally, Table 3.1.4 demonstrates that crude and adjusted results based upon the individual outcomes (stroke, MI, SCA) were not materially different than those for the composite endpoint. 
ADHD Drugs and CV Safety in ADHD and ASD Children

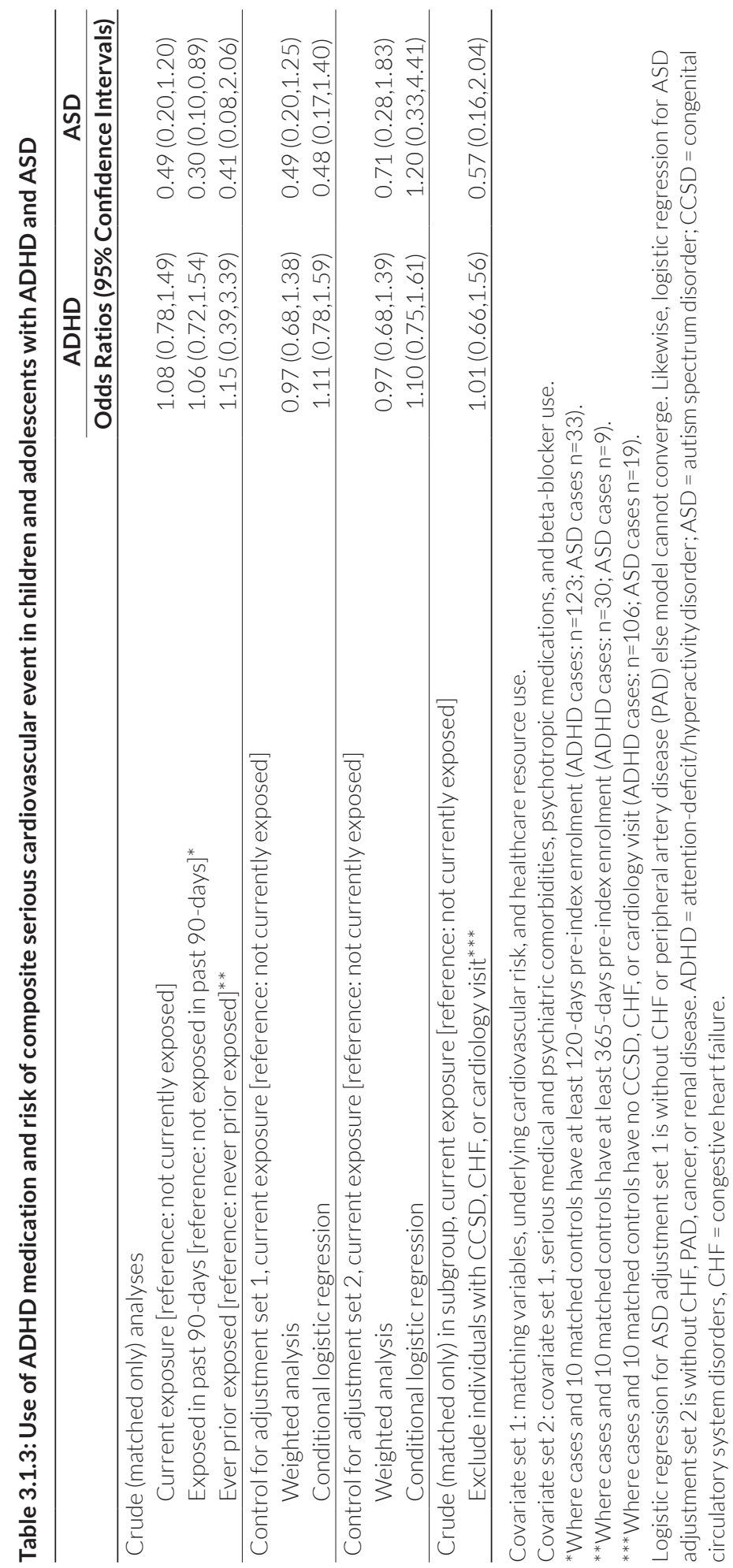




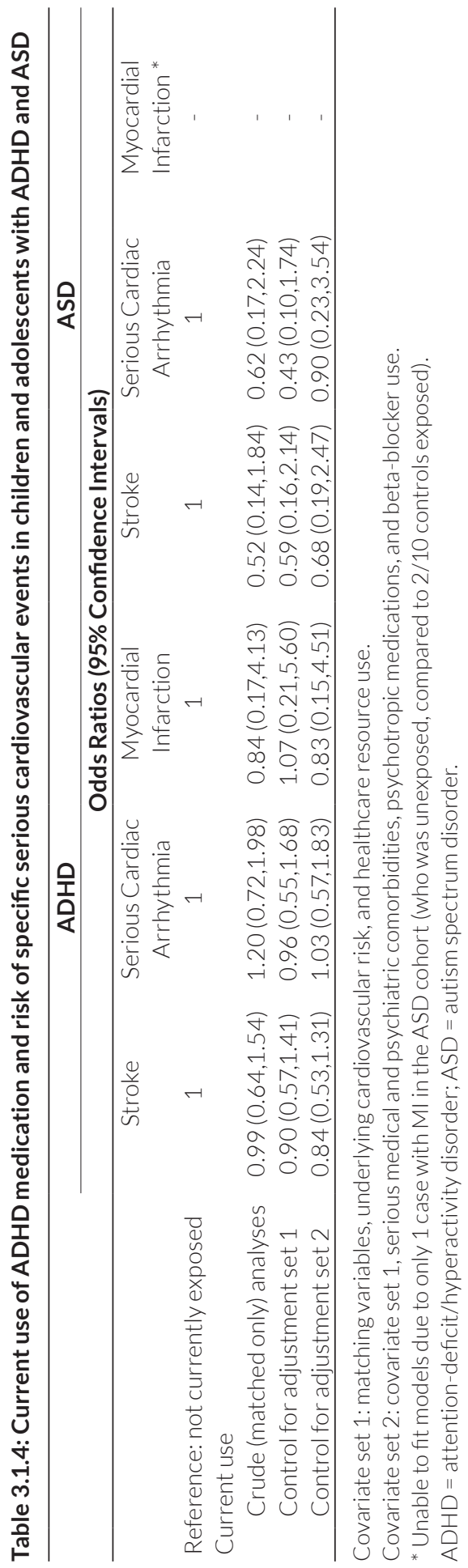




\section{Discussion}

Results of this study indicate that there is no association between the use of ADHD medications and increased risk of SCV events in children and adolescents with ADHD and ASD. Strengths of our study lie in the large number of individuals observed (234 events in total vs 81 in the largest previous study with similar outcomes ${ }^{15}$ ), representivity across all states of the US, and the objectivity of administrative claims data (e.g. no recall bias). Moreover, our results were stable across a series of sensitivity analyses, which adjusted for different covariates (demographics, resource use, comorbidities and concomitant treatment use) and used different statistical models. There was no increased risk found regardless of the timing of exposure, nor for any individual SCV events. Overall, in both cohorts, SCV events were extremely rare.

These findings are largely in line with former research. Indeed, seven of the nine previous studies included in a recent literature review also found no associations between stimulants and paediatric cardiovascular risk ${ }^{14}$. This included three studies perhaps most comparable to ours, also based on US claims data and with similar outcome definitions 15,16,32. Another study in claims data found no associations between current, former, or non-use of stimulants and cardiovascular-related hospitalisations and emergency room visits ${ }^{33}$. The two studies with findings contrary to ours had different outcome definitions. Gould et al ${ }^{34}$ took an unconventional approach in comparing cases of any unexpected deaths to victims of road traffic accidents, while Dalsgaard et al ${ }^{35}$ analysed a cohort of children from Danish national data but used a much wider event definition that included any hospital contact for any cardiovascular reason. A study by Shin et al ${ }^{36}$ found an increased association between methylphenidate use and arrhythmia among children and adolescents in Korea, but again, the definition of arrhythmia was also much wider and included less serious events. No consistent increased risks were found for MI, stroke, heart failure, or hypertension. These data, on the whole are consistent with a recent metaanalysis of methylphenidate, atomoxetine, and/or placebo in controlled trials that showed a pre-post exposure elevation of systolic blood pressure and heart rate in children and adolescents, but no increase of serious cardiac adverse events ${ }^{10}$. In general, these more minor cardiovascular effects during treatment with ADHD medications are thought to be manageable, although should not be underestimated ${ }^{37}$. For consistency with abovementioned previous systematic reviews of ADHD medications on SCV events ${ }^{13,14}$ as well as blood pressure and heart rate 9,10 we did not include guanfacine and clonidine as exposures in our analyses. However, given that these medications have been more recently been approved in some countries for treatment of ADHD, this could be an area for further research.

A novel aspect of our study is the contemporaneous nature of data used (up until the end of 2016). In contrast, the most recent data used by any of the studies included by the Zito and Burcu review ${ }^{14}$ was from 2007, only one year after an FDA advisory committee 
first advised for class-specific warning for stimulants and SCV risk to be introduced ${ }^{12}$. Regardless of these policy statements or subsequent debate about limiting use in people with heart problems ${ }^{38-40}$, inference from our study results remains the same compared to the majority of earlier observational studies: no association found between SCV events and ADHD medications. In any case, across both ADHD and ASD, cases were more likely to already have underlying serious cardiac conditions than controls, which may indicate the class wide warnings are not always followed.

The overall incidence rate of SCV events was extremely low. Our incidence rate estimate of 3.1/100,000 person years in the ADHD cohort is consistent with the rates observed in other cohorts that primarily comprised ADHD children and adolescents (3.1/100,000 15 and 2.8/100,000 ${ }^{32}$ ). Underlying risk for the subgroup of children and adolescents with ASD in our study was slightly higher (5.6/100,000 person years), which may be partially explained by higher prevalence of other psychotropic drugs within this group ${ }^{41}$. The lower point estimate for exposure-outcome relationship found in the ASD group may also be a consequence of their higher concurrent treatment use, with more caution exercised by prescribing doctors deciding if to suggest ADHD medication as an additional treatment, or not.

Designing this study presented different methodological considerations. Due to the expected rarity of events, we used a nested case-control study design to include as many events in the analyses as possible. However, this meant there was possibility of overadjustment via inclusion of post-exposure variables, and hence we emphasised results of the crude matched analyses. When we did adjust for covariates, we tried to mitigate the risk of over-adjustment by not counting medical diagnoses and HCRU variables within the month prior to index. Furthermore, as logistic regression adjustment for many covariates and small sample sizes is known to increase the chance of unstable results ${ }^{42}$, we opted for a weighted analysis as our primary adjusted model. Attaching weights to observations from the control group, such that this group is more similar to the cases, is an extension of simple matching, with the same theoretical motivation. In matched cohort studies, propensity scores are commonly used to find suitable weights, but here we preferred the gradient boosted method because the algorithm directly assigns weights for optimum balance without need to model the propensity of group assignment in the first place. This has two advantages: firstly, that many covariates can be controlled for without considering the functional form of their relationships to each other and to group assignment ${ }^{31}$, and secondly, that there are known difficulties in estimating propensity scores for case-control studies ${ }^{43}$.

Other limitations of our study include the inability to confirm outcomes by linking claims data to medical records, or assessing medication adherence beyond prescription filing, however we expect such misclassifications to be few, non-differential between groups, and have little bearing on our results. The case-control design also limits interpretation 
to the subgroup of ADHD and ASD children and adolescents reflective of those who experience SCV events. Confounding by contraindication means that cases with more severe underlying cardiac conditions and inpatient, emergency and cardiology resource use may actually have been least likely to receive ADHD medication, biasing results away from a positive association. Finally, despite controlling for many factors, it is possible that residual confounding remained, either through unobserved variables (e.g. diet/exercise) or limited detail in the database (e.g. severity of comorbid conditions). Such limitations are common to many epidemiological studies, but since the SCV event rate is low and ADHD medications are widely used, randomised studies to address this question are unpractical, and analysis of large-scale, real world observational data is meaningful and relevant.

In conclusion, in a large, contemporary insurance database, we found low rates of SCV events in children and adolescents with ADHD (3.1/100,000 person years) and ASD (5.6/100,000 person years). Furthermore, we found no evidence of an increased SCV risk when exposed to ADHD medications. 


\section{References}

1. Jobski, K., Höfer, J., Hoffmann, F. \& Bachmann, C. Use of psychotropic drugs in patients with autism spectrum disorders: a systematic review. Acta Psychiatr. Scand. 135, 8-28 (2017).

2. Burcu, M., Zito, J. M., Metcalfe, L., Underwood, H. \& Safer, D. J. Trends in Stimulant Medication Use in Commercially Insured Youths and Adults, 2010-2014. JAMA Psychiatry 73, 992993 (2016).

3. Olfson, M., King, M. \& Schoenbaum, M. Stimulant Treatment of Young People in the United States. J. Child Adolesc. Psychopharmacol. 26, 520-526 (2016).

4. Bachmann, C. J. et al. Trends in ADHD medication use in children and adolescents in five western countries, 2005-2012. Eur. Neuropsychopharmacol. J. Eur. Coll. Neuropsychopharmacol. 27, 484-493 (2017).

5. Hales, C. M., Kit, B. K., Gu, Q. \& Ogden, C. L. Trends in Prescription Medication Use Among Children and AdolescentsUnited States, 1999-2014. JAMA 319, 2009-2020 (2018).

6. Piper, B. J. et al. Trends in use of prescription stimulants in the United States and Territories, 2006 to 2016. PLOS ONE 13, e0206100 (2018).

7. Raman, S. R. et al. Trends in attentiondeficit hyperactivity disorder medication use: a retrospective observational study using population-based databases. Lancet Psychiatry 5, 824-835 (2018).

8. Cortese, S. et al. Comparative efficacy and tolerability of medications for attentiondeficit hyperactivity disorder in children, adolescents, and adults: a systematic review and network meta-analysis. Lancet Psychiatry 5, 727-738 (2018).

9. Hennissen, L. et al. Cardiovascular Effects of Stimulant and NonStimulant Medication for Children and Adolescents with ADHD: A Systematic Review and Meta-Analysis of Trials of Methylphenidate, Amphetamines and Atomoxetine. CNS Drugs 31, 199-215 (2017).

10. Liang, E. F. et al. The Effect of Methylphenidate and Atomoxetine on Heart Rate and Systolic Blood Pressure in Young People and Adults with AttentionDeficit Hyperactivity Disorder (ADHD): Systematic Review, Meta-Analysis, and Meta-Regression. Int. J. Environ. Res. Public. Health 15, (2018).
11. Silva, R. R., Skimming, J. W. \& Muniz, R. Cardiovascular safety of stimulant medications for pediatric attentiondeficit hyperactivity disorder. Clin. Pediatr. (Phila.) 49, 840-851 (2010).

12. Nissen, S. E. ADHD drugs and cardiovascular risk. N. Engl. J. Med. 354 , 1445-1448 (2006).

13. Westover, A. N. \& Halm, E. A. Do prescription stimulants increase the risk of adverse cardiovascular events?: A systematic review. BMC Cardiovasc. Disord. 12, 41 (2012).

14. Zito, J. M. \& Burcu, M. Stimulants and Pediatric Cardiovascular Risk. J. Child Adolesc. Psychopharmacol. 27, 538-545 (2016).

15. Cooper, W. O. et al. ADHD Drugs and Serious Cardiovascular Events in Children and Young Adults. N. Engl. J. Med. 365, 1896-1904 (2011).

16. Schelleman, $H$. et al. Cardiovascular Events and Death in Children Exposed and Unexposed to ADHD Agents. Pediatrics 127, 1102-1110 (2011).

17. Houghton, R., Ong, R. C. \& Bolognani, F. Psychiatric comorbidities and use of psychotropic medications in people with autism spectrum disorder in the United States. Autism Res. 10, 2037-2047 (2017).

18. Oswald, D.P.\&Sonenklar, N.A. Medication use among children with autism spectrum disorders. J. Child Adolesc. Psychopharmacol. 17, 348-355 (2007).

19. Burke, J. P. et al. Does a claims diagnosis of autism mean a true case? Autism Int. J. Res. Pract. 18, 321-330 (2014).

20. Leitner, Y. The Co-Occurrence of Autism and Attention Deficit Hyperactivity Disorder in Children - What Do We Know? Front. Hum. Neurosci. 8, 268 (2014).

21. Zablotsky, B., Bramlett, M.D. \& Blumberg, S. J. The Co-Occurrence of Autism Spectrum Disorder in Children With ADHD. J. Atten. Disord. 24, 94-103 (2020).

22. Choma, N. N. et al. An algorithm to identify incident myocardial infarction using Medicaid data. Pharmacoepidemiol. Drug Saf. 18, 1064-1071 (2009).

23. Habel, L. A. et al. ADHD Medications and Risk of Serious Cardiovascular Events in Young and Middle-aged Adults. JAMA 306, 2673 (2011). 
24. Hennessy, S. et al. Validation of diagnostic codes for outpatient-originating sudden cardiac death and ventricular arrhythmia in Medicaid and Medicare claims data. Pharmacoepidemiol. Drug Saf. 19, 555-562 (2010).

25. Kumamaru, $H$. et al. Validity of claimsbased stroke algorithms in contemporary Medicare data: reasons for geographic and racial differences in stroke (REGARDS) study linked with medicare claims. Circ. Cardiovasc. Qual. Outcomes 7, 611-619 (2014).

26. McCormick, N., Lacaille, D., Bhole, V. \& Avina-Zubieta, J. A. Validity of Myocardial Infarction Diagnoses in Administrative Databases: A Systematic Review. PLOS ONE 9, e92286 (2014).

27. McCormick, N., Bhole, V., Lacaille, D. \& Avina-Zubieta, J. A. Validity of Diagnostic Codes for Acute Stroke in Administrative Databases: A Systematic Review. PLOS ONE 10, e0135834 (2015).

28. Langholz, B. \& Goldstein, L. Risk Set Sampling in Epidemiologic Cohort Studies. Stat. Sci. 11, 35-53 (1996).

29. Greenland, S., Pearl, J. \& Robins, J. M. Causal diagrams for epidemiologic research. Epidemiol. Camb. Mass 10, 37-48 (1999).

30. Textor, J., Hardt, J. \& Knüppel, S. DAGitty: a graphical tool for analyzing causal diagrams. Epidemiol. Camb. Mass 22, 745 (2011).

31. McCaffrey, D. F., Ridgeway, G. \& Morral, A. R. Propensity score estimation with boosted regression for evaluating causal effects in observational studies. Psychol. Methods 9, 403-425 (2004).

32. Winterstein, A. G. et al. Cardiovascular safety of central nervous system stimulants in children and adolescents: population based cohort study. The BMJ 345, e4627 (2012).

33. Olfson, M. et al. Stimulants and Cardiovascular Events in Youth with AttentionDeficit/Hyperactivity Disorder. J. Am. Acad. Child Adolesc. Psychiatry 51, 147156 (2012).

34. Gould, M. S. et al. Sudden death and use of stimulant medications in youths. Am. J. Psychiatry 166, 992-1001 (2009).
35. Dalsgaard, S., Kvist, A. P., Leckman, J. F., Nielsen, H. S. \& Simonsen, M. Cardiovascular Safety of Stimulants in Children with Attention-Deficit/ Hyperactivity Disorder: A Nationwide Prospective Cohort Study. J. Child Adolesc. Psychopharmacol. 24, 302-310 (2014).

36. Shin, J.-Y., Roughead, E. E., Park, B.-J. $\&$ Pratt, N. L. Cardiovascular safety of methylphenidate among children and young people with attention-deficit/ hyperactivity disorder (ADHD): nationwide self controlled case series study. BMJ 353, i2550 (2016).

37. Cortese, S. et al. Practitioner review: current best practice in the management of adverse events during treatment with ADHD medications in children and adolescents. J. Child Psychol. Psychiatry 54, 227-246 (2013).

38. Perrin, J. M., Friedman, R. A., Knilans, T. K., Group, the B. B.W. \& Surgery, the S. on C. and C. Cardiovascular Monitoring and Stimulant Drugs for Attention-Deficit/ Hyperactivity Disorder. Pediatrics 122, 451-453 (2008).

39. Vetter, V. L. et al. Cardiovascular Monitoring of Children and Adolescents With Heart Disease Receiving Medications for Attention Deficit/Hyperactivity Disorder: A Scientific Statement From the American Heart Association Council on Cardiovascular Disease in the Young Congenital Cardiac Defects Committee and the Council on Cardiovascular Nursing. Circulation 117, 2407-2423 (2008).

40. Wilens, T. E., Prince, J. B., Spencer, T. J. $\&$ Biederman, J. Stimulants and sudden death: what is a physician to do? Pediatrics 118, 1215-1219 (2006).

41. Ray, W. A. et al. Association of Antipsychotic Treatment With Risk of Unexpected Death Among Children and Youths. JAMA Psychiatry 76, 162-171 (2019). 42. Peduzzi, P., Concato, J., Kemper, E., Holford, T. R. \& Feinstein, A. R. A simulation study of the number of events per variable in logistic regression analysis. J. Clin. Epidemiol. 49, 1373-1379 (1996).

43. Mansson, R., Joffe, M. M., Sun, W. \& Hennessy, S. On the Estimation and Use of Propensity Scores in Case-Control and Case-Cohort Studies. Am. J. Epidemiol. 166, 332-339 (2007). 
S.3.1.1: Directed acyclic graph for the causal assumptions between exposure (ADHD medication), outcome (serious $\mathrm{CV}$ event), and possible confounders

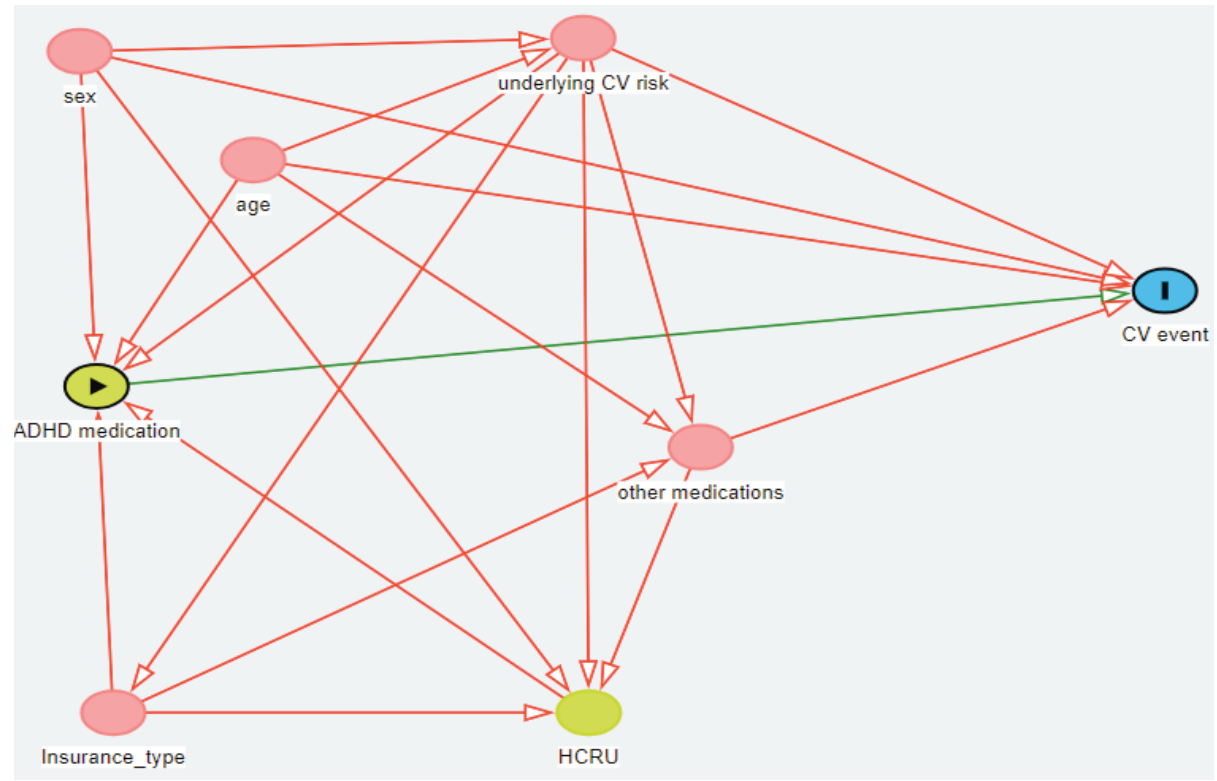

$\mathrm{ADHD}=$ attention-deficit/hyperactivity disorder. $\mathrm{CV}$ event = serious cardiovascular event.

Underlying CV risk = risk from underlying comorbidities.

$\mathrm{HCRU}=$ Healthcare resource utilisation, a proxy for general health status and propensity to use and claim for healthcare interactions. Insurance = commercial vs Medicaid and capitated vs fee-for-service plans.

According to causal diagram theory and our diagram, the minimal efficient set of variables needed for adjustment was sex, age, insurance type, underlying CV risk, and healthcare resource utilisation (HCRU). While sex, age, and insurance type were adjusted for by matching, underlying CV risk and HCRU were included in both covariate adjustments set 1 and set 2 (see methods). 


\section{S.3.1.2: Listing of serious cardiovascular events observed among cases in the ADHD cohort} ( $n=186)$

Stroke $(\mathrm{n}=102)$

$n(\%)$

ICD-9-CM

431: Intracerebral hemorrhage

430: Subarachnoid hemorrhage

434.91: Cerebral artery occlusion, unspecified with cerebral infarction

434.11: Cerebral embolism with cerebral infarction

434.01: Cerebral thrombosis with cerebral infarction

433.11: Occlusion and stenosis of carotid artery with cerebral infarction

433.81: Occlusion and stenosis of other specified precerebral artery with cerebral infarction

ICD-10-CM

161.1: Nontraumatic intracerebral hemorrhage in hemisphere, cortical

163.8: Other cerebral infarction

161.8: Other nontraumatic intracerebral hemorrhage

163.9: Cerebral infarction, unspecified

160.6: Nontraumatic subarachnoid hemorrhage from other intracranial arteries

160.8: Other nontraumatic subarachnoid hemorrhage

161.5: Nontraumatic intracerebral hemorrhage, intraventricular

161.9: Nontraumatic intracerebral hemorrhage, unspecified

163.10: Cerebral infarction due to embolism of unspecified precerebral artery

1633.11: Cerebral infarction due to thrombosis of right middle cerebral artery

163.419: Cerebral infarction due to embolism of unspecified middle cerebral artery

163.511: Cerebral infarction due to unspecified occlusion or stenosis of right middle cerebral artery

163.512: Cerebral infarction due to unspecified occlusion or stenosis of left middle cerebral artery

163.59: Cerebral infarction due to unspecified occlusion or stenosis of other cerebral artery

\section{Myocardial infarction $(n=10)$}

ICD-9-CM

410.71: Subendocardial infarction, initial episode of care

410.01: Acute myocardial infarction of anterolateral wall, initial episode of care

410.41: Acute myocardial infarction of other inferior wall, initial episode of care

410.11: Acute myocardial infarction of other anterior wall, initial episode of care

410.91: Acute myocardial infarction of unspecified site, initial episode of care 
S.3.1.2: Listing of serious cardiovascular events observed among cases in the ADHD cohort ( $n=186)$ (cont.)

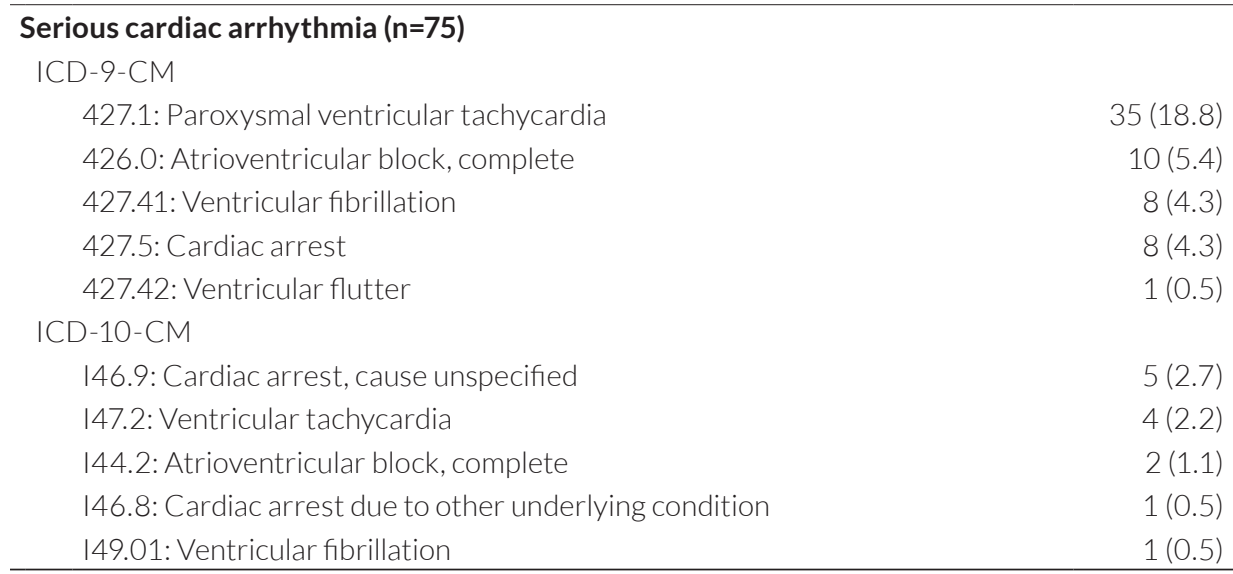

$\mathrm{n}(\%)$ are based on the $\mathrm{n}=186$ composite events. One person had more than one event, namely a stroke (433.11) and serious cardiac arrhythmia (I47.2).ADHD = attention-deficit/hyperactivity disorder. ICD-CM = International Classification of Diseases, Clinical Modification (version 9 and version 10 used). 


\section{S.3.1.3: Listing of serious cardiovascular events observed among cases in the ASD cohort} $(n=48)$

\begin{tabular}{lr}
\hline Stroke (n=25) & $n(\%)$ \\
ICD-9-CM & \\
434.91: Cerebral artery occlusion, unspecified with cerebral infarction & $8(16.7)$ \\
431: Intracerebral hemorrhage & $5(10.4)$ \\
430: Subarachnoid hemorrhage & $4(8.3)$ \\
434.01: Cerebral thrombosis with cerebral infarction & $1(2.1)$ \\
434.11: Cerebral embolism with cerebral infarction & $1(2.1)$ \\
ICD-10-CM & \\
161.1: Nontraumatic intracerebral hemorrhage in hemisphere, cortical & $1(2.1)$ \\
161.5: Nontraumatic intracerebral hemorrhage, intraventricular & $1(2.1)$ \\
163.132: Cerebral infarction due to embolism of left carotid artery & $1(2.1)$ \\
163.232: Cerebral infarction due to unspecified occlusion or stenosis of left carotid & $1(2.1)$ \\
arteries & \\
163.49: Cerebral infarction due to embolism of other cerebral artery & $1(2.1)$ \\
163.532: Cerebral infarction due to unspecified occlusion or stenosis of left posterior & $1(2.1)$ \\
cerebral artery & \\
\hline Myocardial infarction (n=1) & \\
ICD-9-CM & $1(2.1)$ \\
410.41: Acute myocardial infarction of other inferior wall, initial episode of care & \\
\hline Serious cardiac arrhythmia (n=22) & \\
ICD-9-CM & $8(16.7)$ \\
427.1: Paroxysmal ventricular tachycardia & $5(10.4)$ \\
427.5: Cardiac arrest & $3(6.3)$ \\
427.41: Ventricular fibrillation & $2(4.2)$ \\
146.9: Cardiac arrest, cause unspecified & $2(4.2)$ \\
147.2: Ventricular tachycardia & $1(2.1)$ \\
144.2: Atrioventricular block, complete & $1(2.1)$ \\
146.2: Cardiac arrest due to underlying cardiac condition &
\end{tabular}

$\mathrm{n}(\%)$ are based on the $\mathrm{n}=48$ composite events. ASD = autism spectrum disorder.

ICD-CM = International Classification of Diseases, Clinical Modification (version 9 and version 10 used). 


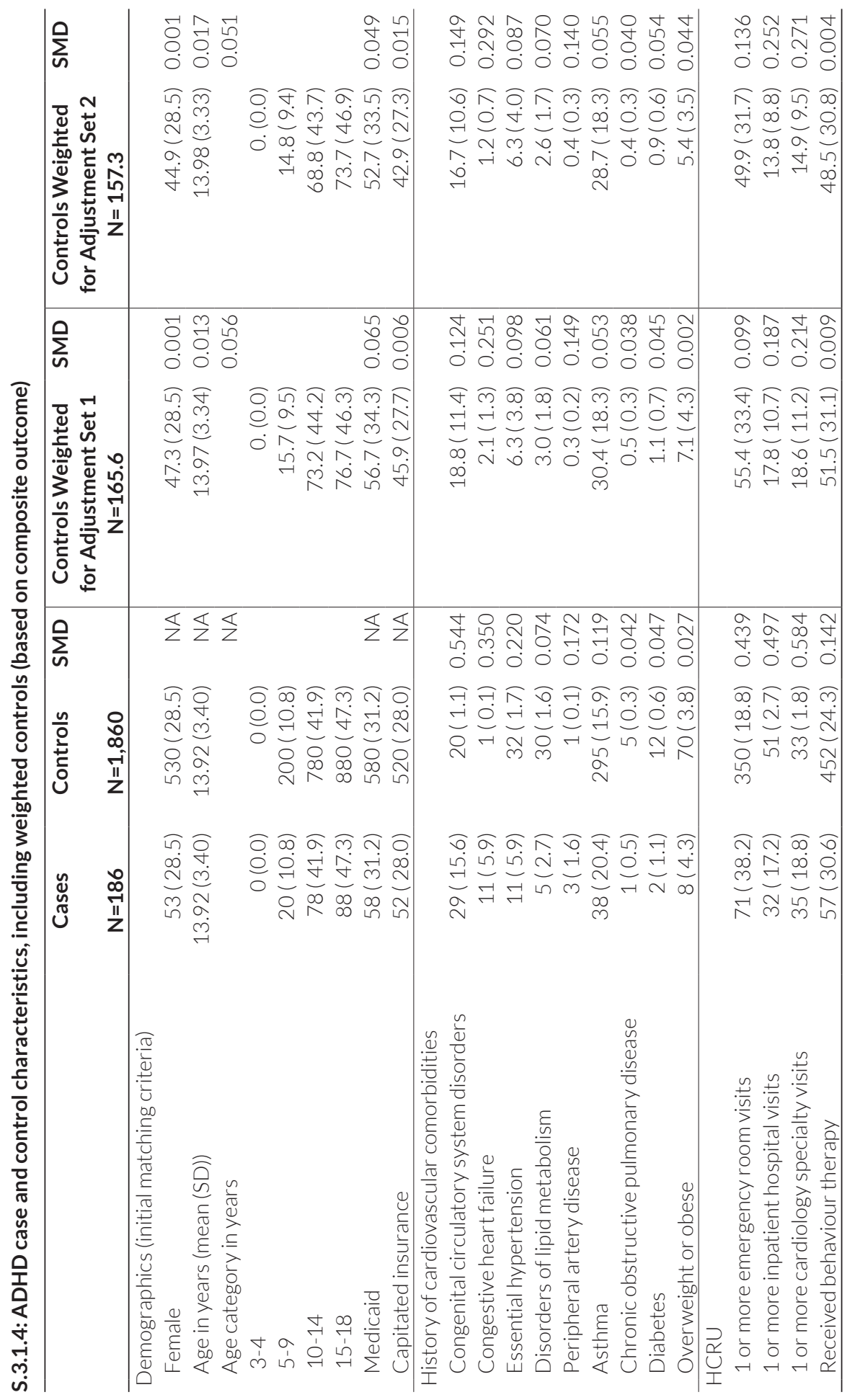




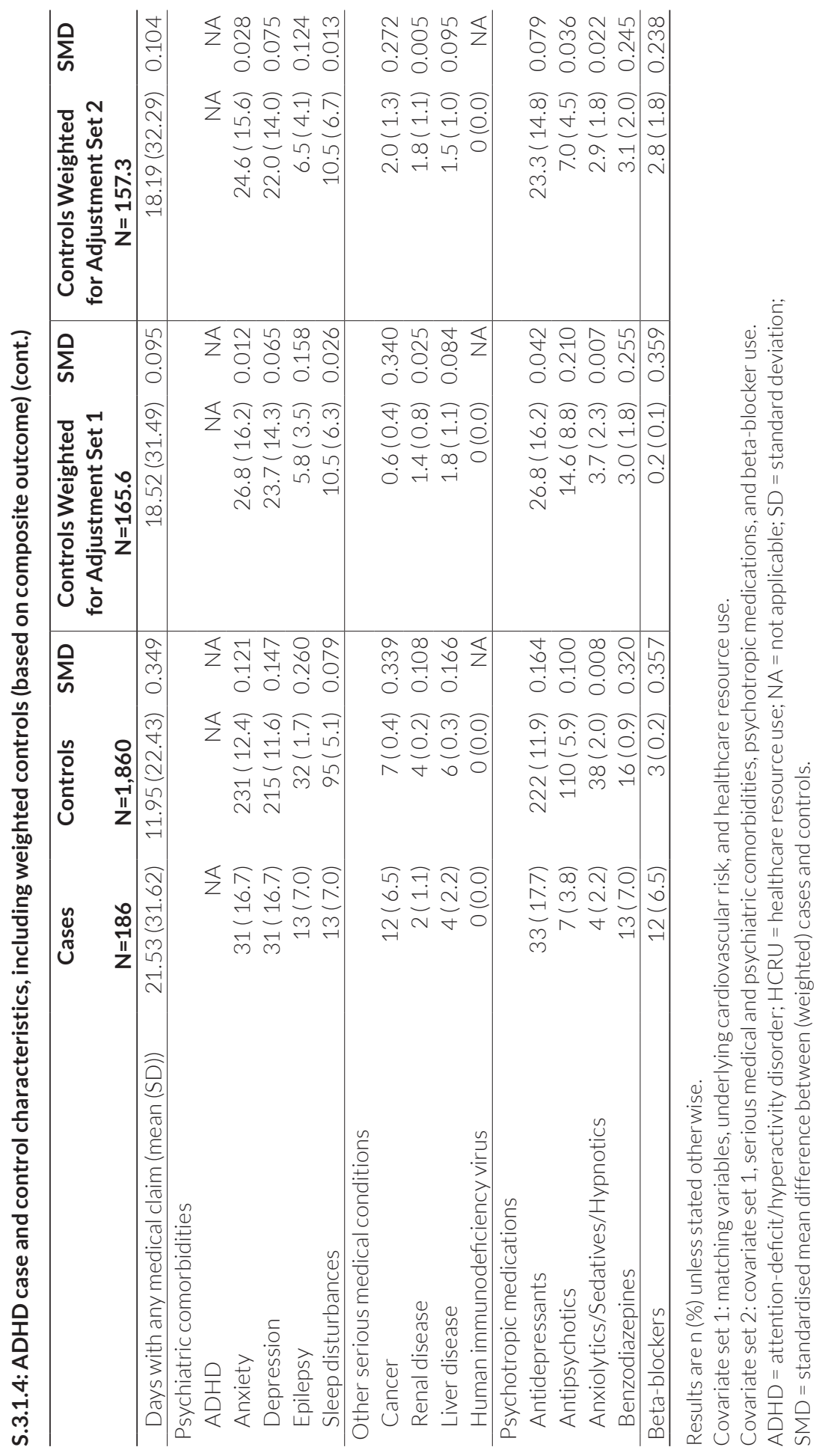




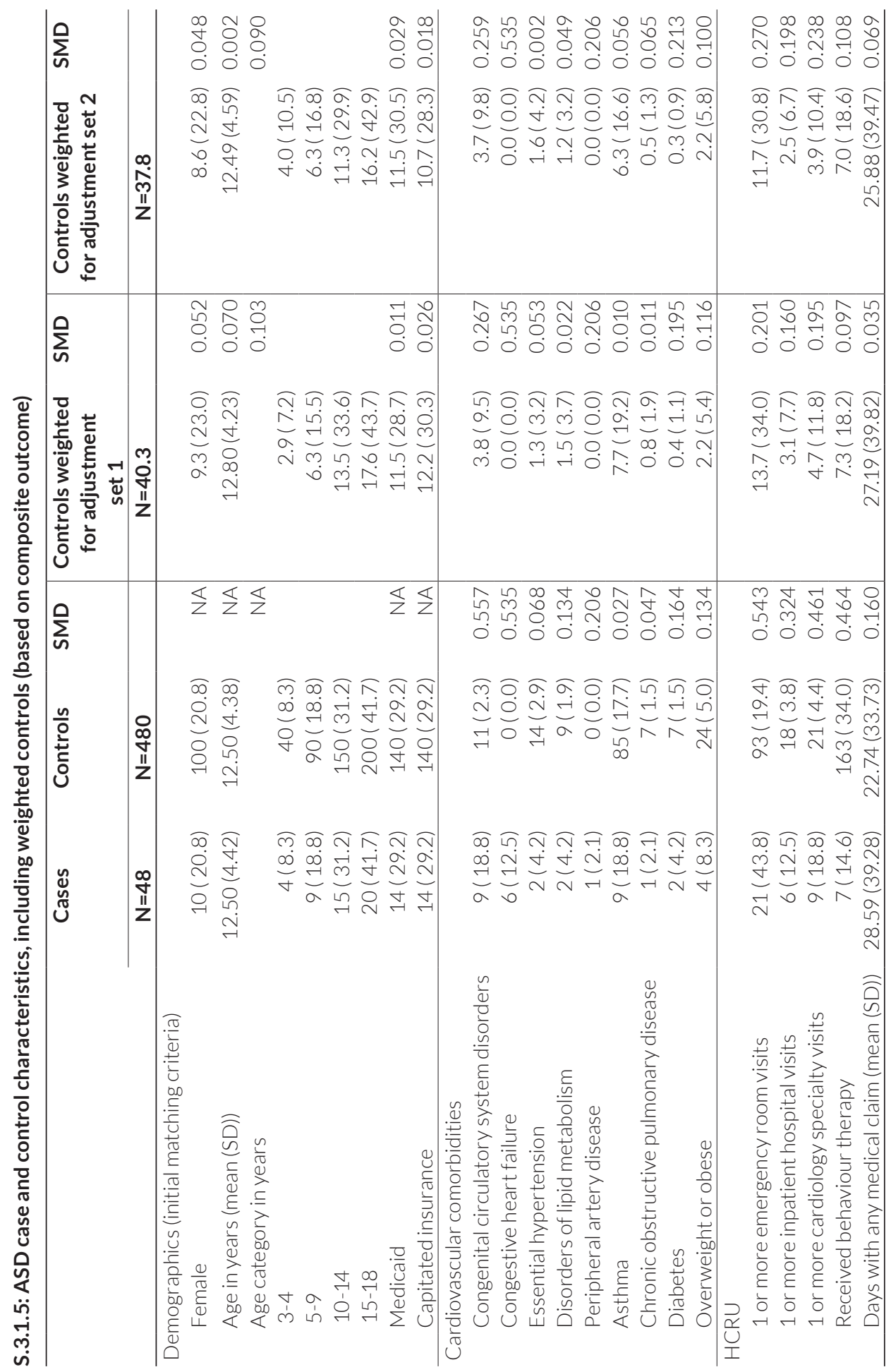




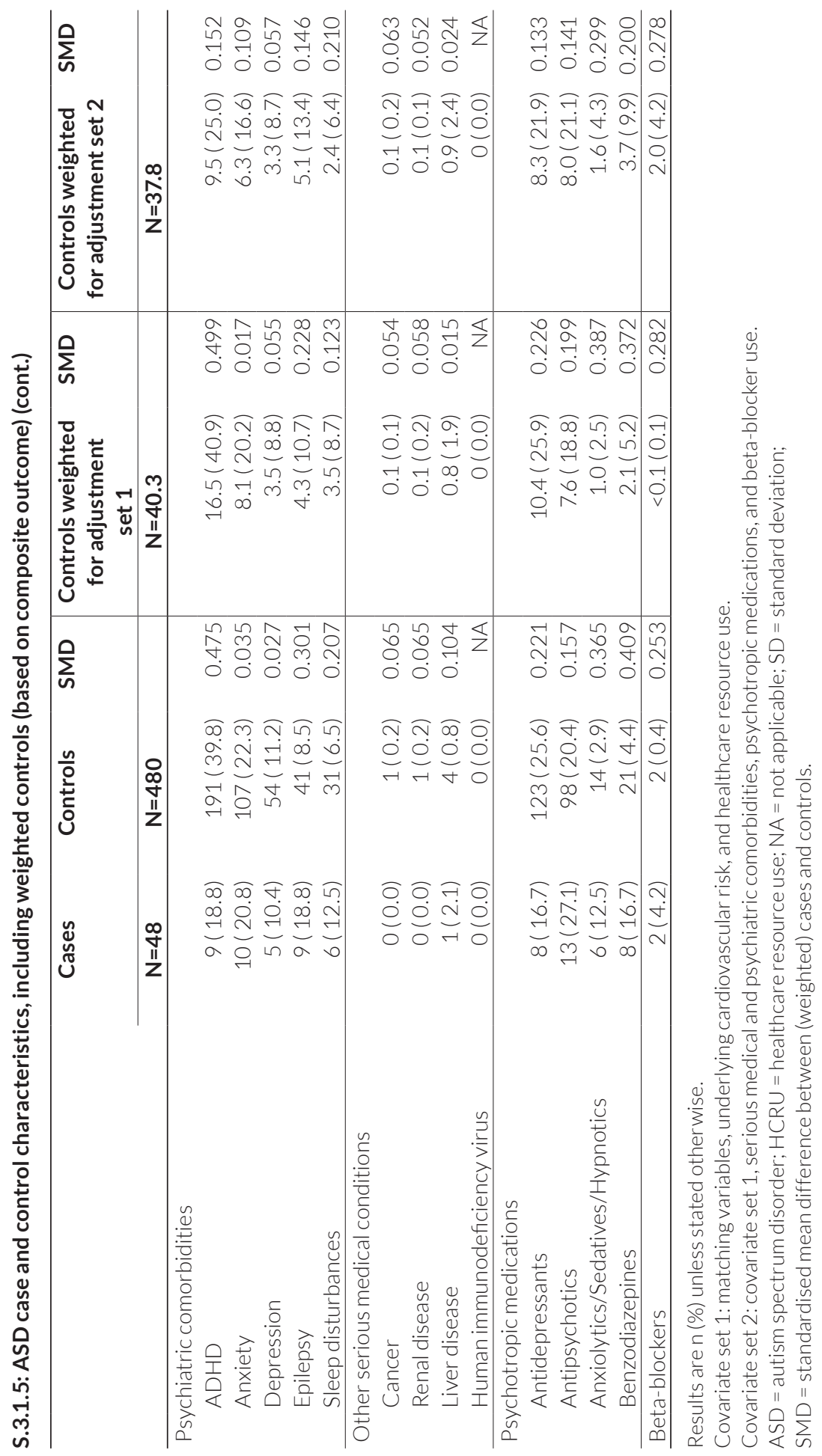


S.3.1.6: Specific ADHD medications and mean doses currently prescribed to cases and controls with ADHD and ASD

\begin{tabular}{|c|c|c|c|c|}
\hline & \multicolumn{2}{|c|}{ ADHD } & \multicolumn{2}{|c|}{ ASD } \\
\hline & $\begin{array}{c}\text { Case } \\
(\mathrm{N}=186)\end{array}$ & $\begin{array}{c}\text { Control } \\
(\mathrm{N}=1,860)\end{array}$ & $\begin{array}{c}\text { Case } \\
(\mathrm{N}=48)\end{array}$ & $\begin{array}{l}\text { Control } \\
(\mathrm{N}=480)\end{array}$ \\
\hline \multicolumn{5}{|l|}{ Atomoxetine } \\
\hline Currently exposed (n, \%) & $2(1.1)$ & $44(2.4)$ & $0(0.0)$ & $12(2.5)$ \\
\hline Dose, mg/day (mean, SD) & $60.00(28.28)$ & $45.84(22.23)$ & NA & $55.14(28.10)$ \\
\hline \multicolumn{5}{|l|}{ Amphetamine } \\
\hline Currently exposed (n, \%) & $17(9.1)$ & $138(7.4)$ & $1(2.1)$ & $15(3.1)$ \\
\hline Dose, mg/day (mean, SD) & $22.21(16.00)$ & $24.03(13.85)$ & 10.00 (NA) & $19.29(10.72)$ \\
\hline \multicolumn{5}{|l|}{ Dexmethylphenidate } \\
\hline Currently exposed (n, \%) & $7(3.8)$ & $59(3.2)$ & $3(6.2)$ & $11(2.3)$ \\
\hline Dose, mg/day (mean, SD) & $12.86(4.88)$ & $16.43(9.89)$ & $27.50(17.68)$ & $15.23(8.25)$ \\
\hline \multicolumn{5}{|l|}{ Lisdexamfetamine } \\
\hline Currently exposed (n, \%) & $8(4.3)$ & $121(6.5)$ & $1(2.1)$ & $24(5.0)$ \\
\hline Dose, mg/day (mean, SD) & $41.25(11.26)$ & $41.73(17.50)$ & 30.00 (NA) & $39.59(19.88)$ \\
\hline \multicolumn{5}{|l|}{ Methylphenidate } \\
\hline Currently exposed (n, \%) & $28(15.1)$ & $232(12.4)$ & $1(2.1)$ & $44(9.1)$ \\
\hline Dose, mg/day (mean, SD) & $36.54(18.67)$ & $35.71(18.69)$ & 10.00 (NA) & $34.55(17.98)$ \\
\hline
\end{tabular}

Dextroamphetamine was the other stimulant prescribed; to 5 individuals with ADHD (1 case and 4 controls). $\mathrm{ADHD}=$ attention-deficit/hyperactivity disorder; $\mathrm{ASD}=$ autism spectrum disorder; NA = not applicable; $\mathrm{SD}=$ standard deviation. 
ADHD Drugs and CV Safety in ADHD and ASD Children 
This chapter is embargoed at request

\section{Chapter 3.2}

Risperidone versus aripiprazole fracture risk in children and adolesce
autism spectrum disorder

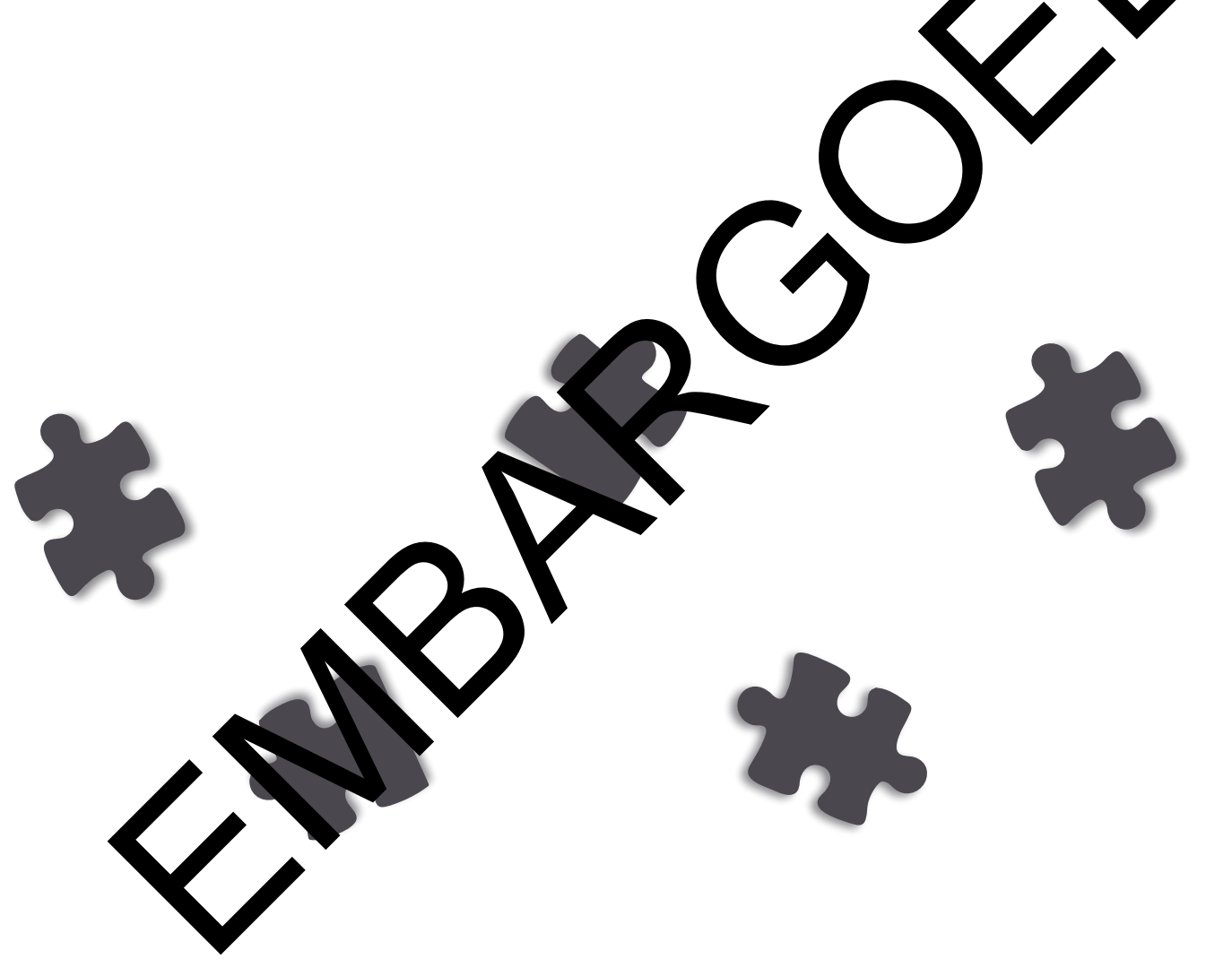

Richard Houghton Joop van den Bergh

Kiely Law Yutong Liu Frank de Vries 



\section{Chapter 4}

Validation of caregiver-reported severity of autism spectrum disorder 


\section{Chapter 4.1}

Psychometric validation of the Autism Impact Measure (AIM)
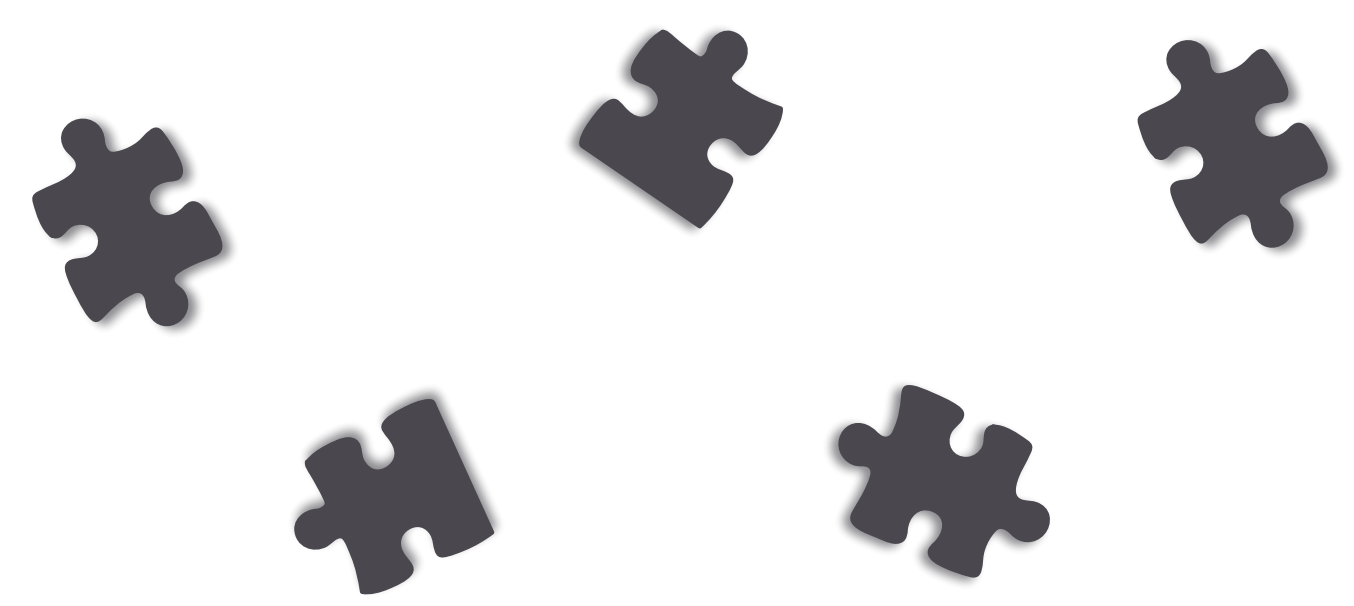

Richard Houghton

Brigitta Monz Kiely Law

Georg Loss

Stephanie Le Scouiller

Frank de Vries

Tom Willgoss 


\section{Abstract}

The Autism impact measure (AIM) is a caregiver-reported questionnaire assessing autism symptom frequency and impact in children, previously shown to have good test-retest reliability, convergent validity and structural validity. This study extended previous work by exploring the AIM's ability to discriminate between "known-groups" of children, and estimating thresholds for clinically important responses. Data were collected online and electronically on computer and mobile devices; hence, it was also possible to confirm other psychometric properties of the AIM in this format. This study provides confirmatory and additional psychometric validation of the AIM. The AIM offers a valid, quick and inexpensive method for caregivers to report core symptoms of autism spectrum disorder (ASD) including communication deficits, difficulties with social interactions and repetitive behaviours.

\section{Lay summary}

The Autism impact measure (AIM) is a caregiver-reported questionnaire to assess autism symptom frequency and impact in children. This study showed the AIM is a valid tool, which can be quickly completed by caregivers on computer and mobile devices. 


\section{Introduction}

The Autism Impact Measure (AIM) is a caregiver-reported questionnaire, designed to be used in clinical trials and clinical practice to assess effectiveness of interventions in Autism Spectrum Disorders (ASD) 1,2. It consists of 41 items, and each is rated on a 5-point Likert-type scale for both frequency and impact. Caregiver-reported clinical outcome assessment measures such as the AIM may have some advantages over established interview-administered measures in that they are often less time consuming and do not need specifically trained personnel to administer. As a result, the AIM could also potentially serve as suitable tool for real world monitoring of ASD symptoms, embedded in more routine care or remote settings. Additional advantages of the AIM are that other commonly used scales have either been created for diagnostic purposes only (e.g. Autism Diagnostic Observational Scale: ADOS ${ }^{3}$ ), were developed and tested according to older and more narrow definitions of ASD (e.g. Behavioral Summarized Evaluation Scale: BSE ${ }^{4}$ and Real Life Rating Scale: RLRS ${ }^{5}$ ) or focus on non-core or not all core characteristics (e.g. Social responsiveness scale: SRS-2 ${ }^{6}$ ). The AIM, in contrast, has been shown to exhibit 5 "theoretically and empirically meaningful" symptom domains, namely; Repetitive Behavior, Communication, Atypical Behavior, Social Reciprocity and Peer Interaction 2 ${ }^{2}$ The domain scores utilise only 29 of the 41 items, while the total score still builds on all items. Higher domain and total scores represent worse severity of ASD symptoms.

While the AIM has shown to have good test-retest reliability, cross-informant reliability and convergent validity with other scales ${ }^{1,2}$, other important validation questions remain untested. Importantly, the ability of the AIM to detect differences between known subgroups of individuals with ASD has not been demonstrated. Known-group analysis is needed to demonstrate that a measure is sensitive and able to discriminate between subgroups previously established to have differences in severity. Furthermore, there has been no attempt to estimate magnitudes of such differences that constitute clinically meaningful changes. Successful validation of these two concepts is fundamental for confidence to use the AIM in any study wishing to demonstrate efficacy of a given intervention. Therefore, the primary objective of our study was to address these gaps in a large and representative sample. Also, because participants in our study completed the questionnaire electronically, rather than on paper, secondary objectives were to assess the time needed to complete the AIM and confirm other measures of psychometric validity in this format, including internal/external validity and confirmatory factor analysis.

\section{Methods}

\section{Data Collection}

Participants were invited to take part in our study via the Simons Foundation Powering Autism Research for Knowledge (SPARK) cohort. SPARK is an online community for 
people with ASD and their families in the United States (US), who are interested in participating in ASD research ? . Families complete a battery of questionnaires on entry to the cohort, and third-party researchers (industry or academic) can recruit the same families to their studies thereafter. All data generated are anonymised and made linkable via unique identifiers. To be eligible for the current study, participants had to be the main caregiver living in the same household as a child with ASD, and were instructed to answer the AIM in relation to only the oldest child with ASD between 3-17 years. All data used for the study were provided by caregiver-report and were collected during September and October 2017 as part of a wider study on non-drug treatments and potential barriers to care. Details on recruitment and data collection have been published elsewhere ${ }^{8}$. Informed consent was obtained from all individual participants included in the study. The study protocol was approved by Western IRB.

\section{Analysis Populations}

In total, 5,001 participants returned the AIM survey. As the current AIM scoring system does not mention methods for handling missing data, our main analysis population of interest was those 4,415 participants who completed all items. We qualitatively checked for differences in characteristics of those who did not complete all items however, as well as between those who took part on either a vertical or horizontal layout. In the horizontal layout, possible responses to each item were displayed "across" the screen. In the vertical layout the possible responses were displayed "down" the screen (i.e. underneath one another). The format deployed was based on the screen dimensions of the device used to complete the survey (e.g. vertical layout for most mobile phones and horizontal layout for laptops/computers).

A subgroup of respondents had also previously completed the Social Communication Questionnaire-Lifetime (SCQ) ${ }^{9,10}$ and/or the Repetitive Behaviors Sale-Revised (RBS-R) ${ }^{11}$ as part of the SPARK procedures. We linked this data for convergent validity analysis so long as the age of the child differed by no more than 1 year between the time of AIM assessment and the time of SCQ/RBS-R (exact date of SCQ/RBS-R was unknown). Linked sample sizes available were 3,064 for the SCQ and 3,190 for the RBS-R. There was a significant overlap of 2,571 participants who completed all of AIM, SCQ and RBS-R.

\section{Descriptive analysis}

We calculated the mean and median score for all items in order to identify items with higher or lower than average impact and frequency and to assess response distributions. For the purposes of this descriptive analysis, we highlighted items with 50\% or more of responses at the lowest or highest possible values as the cut-off value for which some items might be considered to show floor or ceiling characteristics, respectively. We also assessed missingness for each item and the time taken to complete the AIM. All descriptive analyses were also stratified by vertical/horizontal format. 


\section{Internal consistency}

To measure consistency of underlying concepts, Cronbach's alpha ${ }^{12}$ was calculated for each of the domain scores. We specified a threshold of $\geq 0.7^{13}$ to identify domains with a good internal consistency. Similarly, we also calculated Cronbach's alpha for total AIM score and total scores based on just frequency items or impact items in order to assess whether AIM items contributing to a specific score measured the same construct. We calculated inter-domain correlations using Spearman's correlation coefficient.

\section{Convergent validity}

Pearson correlation coefficients were derived to assess the correlations between total SCQ and total AIM scores, as well as between the total SCQ and different domains of the AIM, and the domain scores of both. This approach was repeated for the RBS-R scores. For the SCQ, three domain scores were derived from item responses as per the SCQ scoring manual ${ }^{10}$. The domains are Reciprocal Social Interaction, Communication and Repetition/Stereotyped Behavior. For RBS-R, factor analysis supports a total score, but also 2-, 3-, 4-, 5- and 6-factor solutions for domain-level scores, but this study utilised the 3 -factor solution, which appears to have the most relevant conceptual structure ${ }^{11}$ : Stereotypy Restricted; Self-injurious; Compulsive, Ritualistic, Sameness.

We expected at least a moderate correlation (>0.3) between the total SCQ/RBS-R and total AIM scores, as well as between the total SCQ/RBS-R and each of the AIM domain scores. Those domains for which we hypothesised the highest correlations (>0.5) have been marked alongside the results for all domains in Table 4.1.3. Our hypotheses were based on domains which were conceptually related. Post-hoc, we recalculated correlations between SCQ and AIM communication domains within certain subgroups. The subgroups of interest were ASD individuals who were verbal or non-verbal only, as this limits the scoring range of the SCQ communication domain score, as well as those aged 4-5 years old, as this is the age range asked to focus on for half of the items of the SCQ: the other half have a lifetime perspective, e.g. "ever had" ${ }^{10}$. In comparison, the RBS-R has no specified recall period and the AIM has a two-week recall period.

\section{Factor analysis}

We summed frequency and impact scores for each of the 29 items which are needed to create the 5 domain scores proposed by the scale developers ${ }^{2}$. We then fitted a 5 -factor solution on those 29 items with Varimax rotation. Finally, we compared items with highest loadings on each factor in our solution, with the domains proposed. The purpose of our factor analysis was only to confirm the five domains suggested by the developers rather than to explore other potential factor solutions. 


\section{Known-groups analysis}

Based on previous literature and clinical knowledge, pre-specified "known-groups" were defined based on the following variables: (1) IQ score ${ }^{14,15}$; (2) proportion of school-time spent with typically developing peers ${ }^{16,17}$; (3) presence/absence of psychiatric comorbidity ${ }_{18}^{18}$; (4) received speech and language therapy (SLT) in the preceding 12-months (particularly relevant for communication domain); (5) caregiver reported overall health status of child (expected to be correlated with ASD severity if caregiver deems ASD symptoms relevant to overall health); (6) children who qualified for Medicaid despite family income greater than $\$ 75,000$ per annum (to identify the subgroup who were Medicaid-eligible based on severity opposed to financial circumstance); (7) the number of non-drug therapies received for ASD in last 12 months 16,17; (8) medication prescribed for ASD (assuming prescriptions are made for individuals with more severe symptoms, on average); (9) verbal/ non-verbal ability (based on item 1 of the SCQ). More detailed definitions of these knowngroups are provided in Table 4.1.1.

We summarised mean and median scores within each level of each known-group and conducted analysis of variance (ANOVA) to see if those differences were statistically significant $(p<0.05)$. We produced both crude and age-adjusted ANOVA results based on the total AIM scores, total frequency/impact scores and individual domain scores.

The range of possible scores for the total AIM was 82-410. For both frequency and impact domains the possible range was 41-205. For each of the domains, the possible ranges were: 16-80 for Repetitive Behavior; 12-60 for Communication; 12-60 for Atypical Behavior; 10-50 for Social Reciprocity; 8-40 for Peer Interaction.

\section{Clinically important responder (CIR) estimates}

As data were collected cross-sectionally we estimated clinically important responder (CIR) thresholds ${ }^{19}$ for the total AIM scores and domain scores using distribution-based methods. Specifically the estimates were based on one-fifth and one-half of standard deviations ${ }^{20,21}$. Prior to generating estimates, we rescaled the maximum range of total and domains scores to 0-100 points. This was done in order to make the magnitude of CIR estimates easier to compare across domains. For completeness we also presented CIR estimates based on raw scores, and we repeated the analysis by age and IQ strata to check for homoscedasticity.

\section{Results}

\section{Cohorts and descriptive analysis}

Figure 4.1.1 and Table 4.1.1 display the flow chart of key study groups and their characteristics, respectively. The majority of participants completed all items $(n=4,415$; 88.3\%). This "completers" group was used as the main analysis group. Around two-thirds of completers (66.4\%) took part in the AIM in vertical layout. 


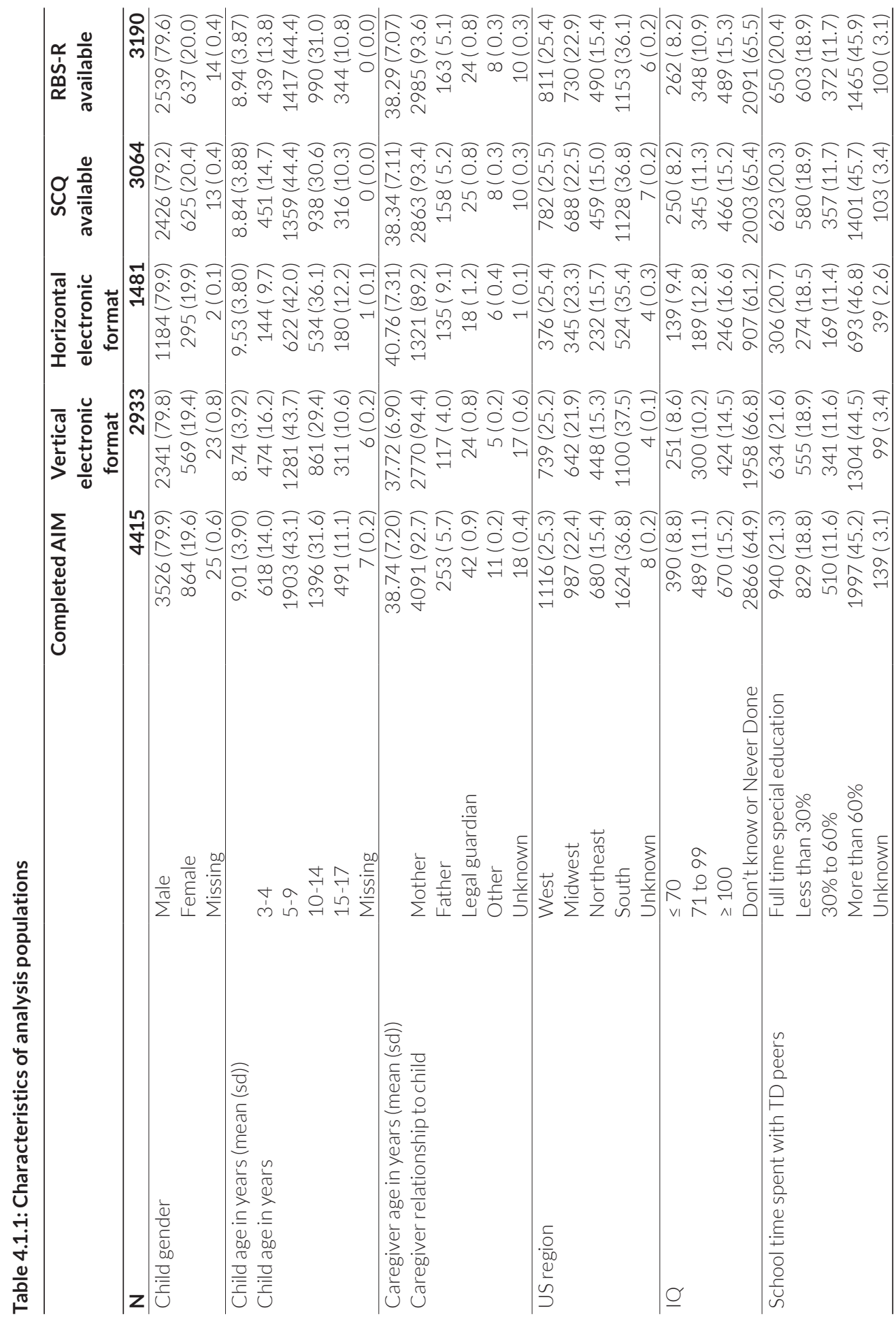




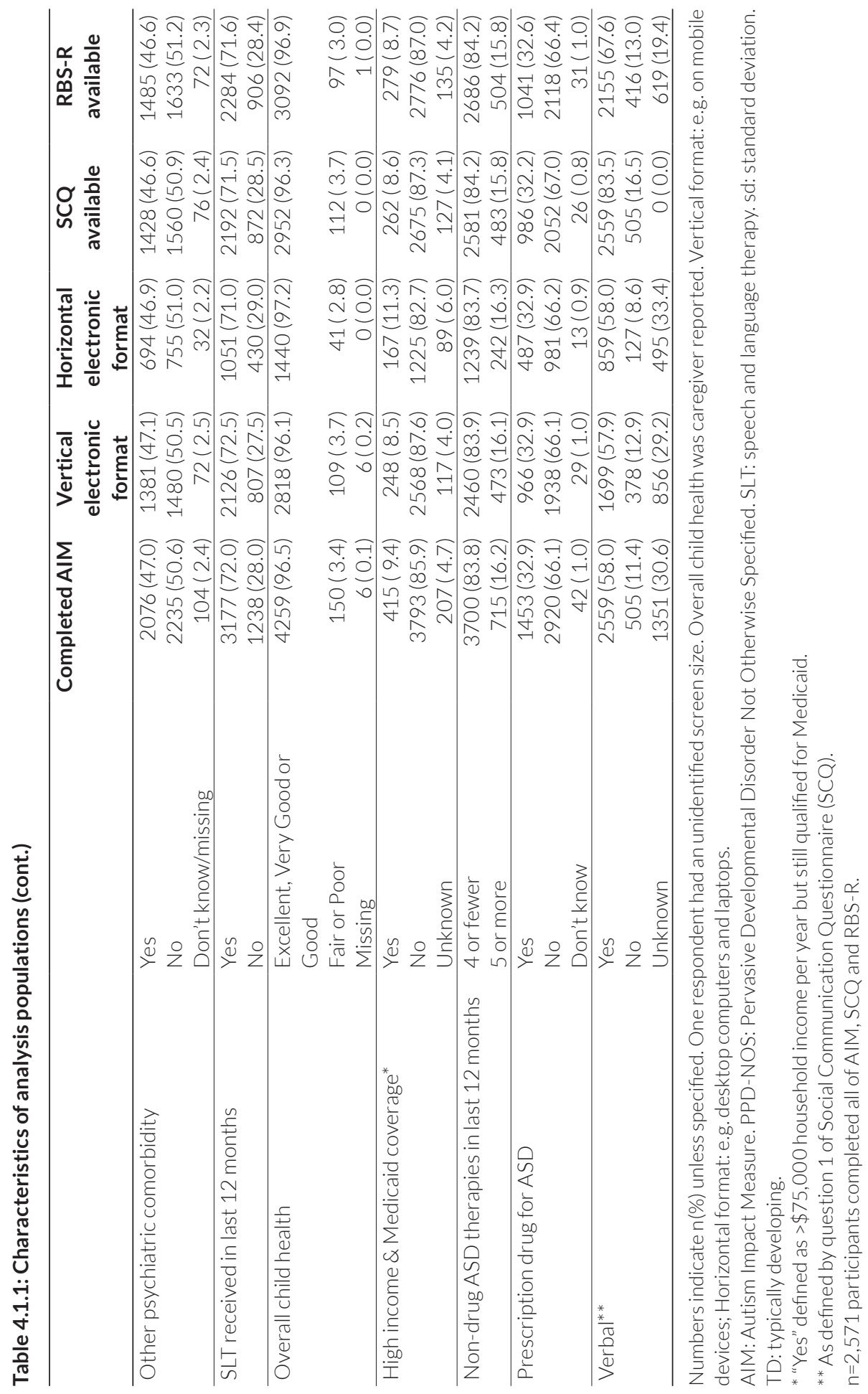


Figure 4.1.1: Flow chart of analysis populations

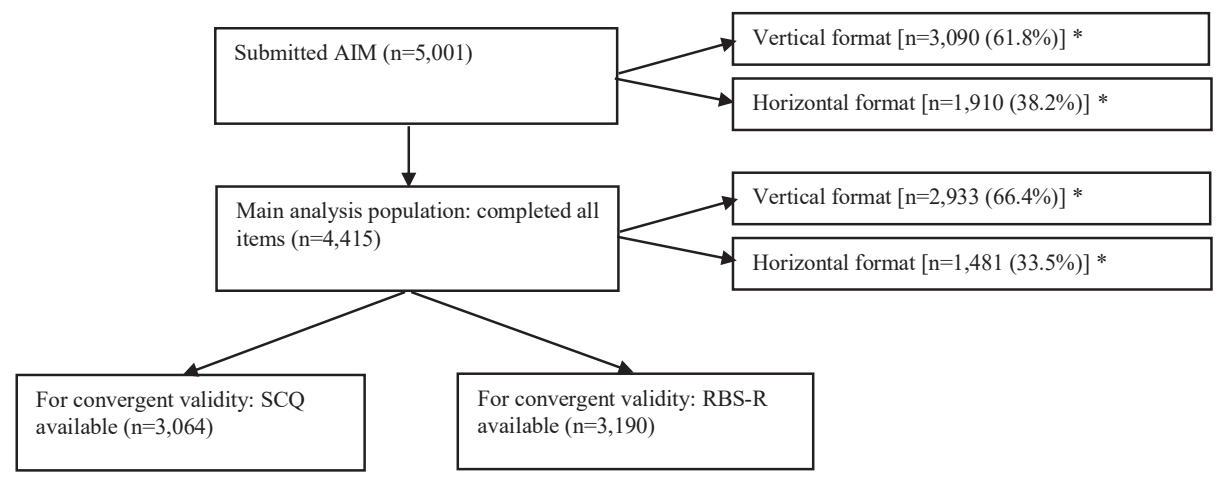

* One respondent had an unidentified screen size. AIM: Autism Impact Measure.

Respondents with complete AIM were mainly mothers (92.7\%) with a mean (SD) age 38.74 (7.20) years. All 50 states of the US were represented as well as some overseas territories. Children with ASD had a mean (SD) age of 9.01 (3.90) and were mainly male (79.9\%). Almost a quarter of children (23.1\%) attended full time special education school, while $45.2 \%$ spent between $60-100 \%$ of school time with typically developing peers. Of those with SCQ available, 83.5\% were verbal (according to item 1 of the SCQ). The only qualitatively notable difference between caregivers who used the vertical instead of horizontal format was their slightly younger mean age (37.7 vs 40.8 years). Furthermore, there were no notable differences for completers, non-completers, and those which had SCQ and/or RBS-R data available for linkage.

The median time to complete the AIM was 7.08 minutes [IQR 5.53 - 9.82]. The mean time was just over one minute faster for completers on the horizontal format (median [IQR] 6.28 minutes [4.90 - 8.63]) versus the vertical format (median [IQR] 7.47 minutes [5.97 - 10.45]). A minority (4.1\% in both vertical and horizontal format) took over one hour to complete all questions.

\section{Item level analysis}

Full item level analyses are summarised in supplement S.4.1.1. Responses to most items were approximately normally distributed. None of the items had a ceiling effect, but 5 had a floor effect which was defined by a median response of 1. Namely these items were: Q3 "lined things up" [impact only, repetitive behavior domain]: Q5 "used hand over hand" [frequency and impact; communication domain]: Q27 "used made-up or private language" [frequency and impact; communication domain].

Disregarding missing values, the item with highest (most severe) mean score (3.90) was Q38 "engaged in chit-chat [frequency; Social Reciprocity domain]. Furthermore, the top 
5 highest scoring items were all frequency questions and only 3 of the top 20 highest scoring (mean 23.02 ) were impact questions. Only 2 of the 20 lowest scoring items (mean $\leq 2.28$ ) were frequency related. Mean scores for each item were not systematically higher or lower based on the vertical or horizontal layout.

Overall, there was very little missing data on an item-by-item basis. Some questions had as little as 10 missed responses from the whole sample (0.20\%). Q36 "showed interest in others" [impact] was most frequently missed but still only for 76 participants (1.52\%). All items were more often missing on the horizontal format, however with $2.46 \%$ being the highest rate of missing data in this layout (Q36 impact). In general, impact questions were more commonly missing than frequency questions.

\section{Internal consistency}

Cronbach alpha for the total AIM score was 0.96, which is well above the threshold of 0.7, which we pre-specified would identify scores with a good internal consistency. Frequency items and impact items also showed high internal consistency (0.96 and 0.95 respectively), as did each of the individual domains (from 0.79 for Social Reciprocity to 0.91 for Communication). The median (IQR) of all inter-item correlations was $r=0.15$ (0.22 - 0.30) and only the correlation between frequency and impact scores for Q6 "problems with speech" was higher than 0.90. These results indicate little item redundancy.

All domains were positively and moderately inter-related according to Spearman's rank coefficient (Table 4.1.2). The weakest relationship was between Repetitive Behavior and Social Reciprocity (0.39). The strongest relationship was between Repetitive Behavior and Atypical Behavior (0.67). Domain correlations were very similar with both Spearman and Pearson correlation methods, indicating that relationships between domain scores were linear.

Table 4.1.2: AIM inter-domain Spearman-rank correlations

\begin{tabular}{lccccc}
\hline & $\begin{array}{c}\text { Repetitive } \\
\text { Behavior }\end{array}$ & Communication & $\begin{array}{c}\text { Atypical } \\
\text { Behavior }\end{array}$ & $\begin{array}{c}\text { Social } \\
\text { Reciprocity }\end{array}$ & $\begin{array}{c}\text { Peer } \\
\text { Interaction }\end{array}$ \\
\hline Repetitive Behavior & - & 0.52 & 0.67 & 0.39 & 0.43 \\
Communication & & - & 0.45 & 0.54 & 0.48 \\
Atypical Behavior & & & - & 0.51 & 0.58 \\
Social Reciprocity & & & & - & 0.63 \\
\hline
\end{tabular}

AIM: Autism Impact Measure. 


\section{Convergent validity}

The total AIM score showed good convergent validity with the total SCQ score $(r=0.55$, Table 4.1.3). Each individual AIM domain was also positively correlated ( $r \geq 0.34)$ with the total SCQ score. As hypothesised, the SCQ Reciprocal Social Interaction domain has highest correlations with the AIM Social Reciprocity (0.48) and Peer Interaction (0.45) domains. Also as expected, the SCQ Repetition/Stereotyped Behavior domain had the strongest relationship with AIM domains of Repetitive Behavior (0.48) and Atypical Behavior (0.34). None of the SCQ-AIM domain-domain relationships met the threshold of 0.5 however, and specifically against our expectations, the SCQ Communication domain was least correlated with the AIM Communication domain (0.18). In sensitivity analyses, this correlation was raised to 0.34 in verbal children and 0.25 in non-verbal children. When restricting to a 4 to 5 years old age-range, the correlation was 0.19.

The RBS-R total score had a strong positive correlation with the total AIM score (0.64). It also had good correlation ( $\geq 0.30$ ) with all AIM domains, frequency and impact scores. Furthermore, for the RBS-R and AIM, all domain-domain correlations were positive, and were strongest (between 0.51 and 0.74 ) in the 4 pre-hypothesised cases. Results for both SCQ and RBS-R remained stable when restricting the analysis population to those children who were exactly the same age (in years) at the time of SCQ/RBS-R and AIM (opposed to within 1-year, as per main analyses; see supplement S.4.1.3).

\section{Factor analysis}

Table 4.1.4 provides a detailed comparison of the proposed factors ${ }^{2}$ and factors found in our confirmatory analysis. The Communication domain was replicated perfectly in our data. The proposed six items for this domain all loaded highest on the third factor produced by our data and no other item loaded highest on this same factor. Other well pronounced and well reproduced latent concepts were Repetitive Behavior and Social Reciprocity. All items proposed for these domains loaded highest on factor 1 and factor 2 in our data, respectively. The only additional item with highest loading on factor 2 was Q32 "had positive response to approach", which was supposed to be part of the Peer Interaction domain. Q32 also had a high loading on factor 4 however, and factor 4 otherwise only had highest loadings of the other 3 of the 4 items representing the Peer Interaction domain. Hence, Peer Interaction was also well reproduced as a latent variable. Finally, 3 of the 6 items expected to load together to form the Atypical Behavior domain indeed did load together in a distinct fifth factor. The other three items however loaded highest on factor 1 , showing some similarity with the Repetitive Behavior concept. The first three factors collectively explained $37.1 \%$ of total variance in the data. Five factors explained $48.4 \%$. 


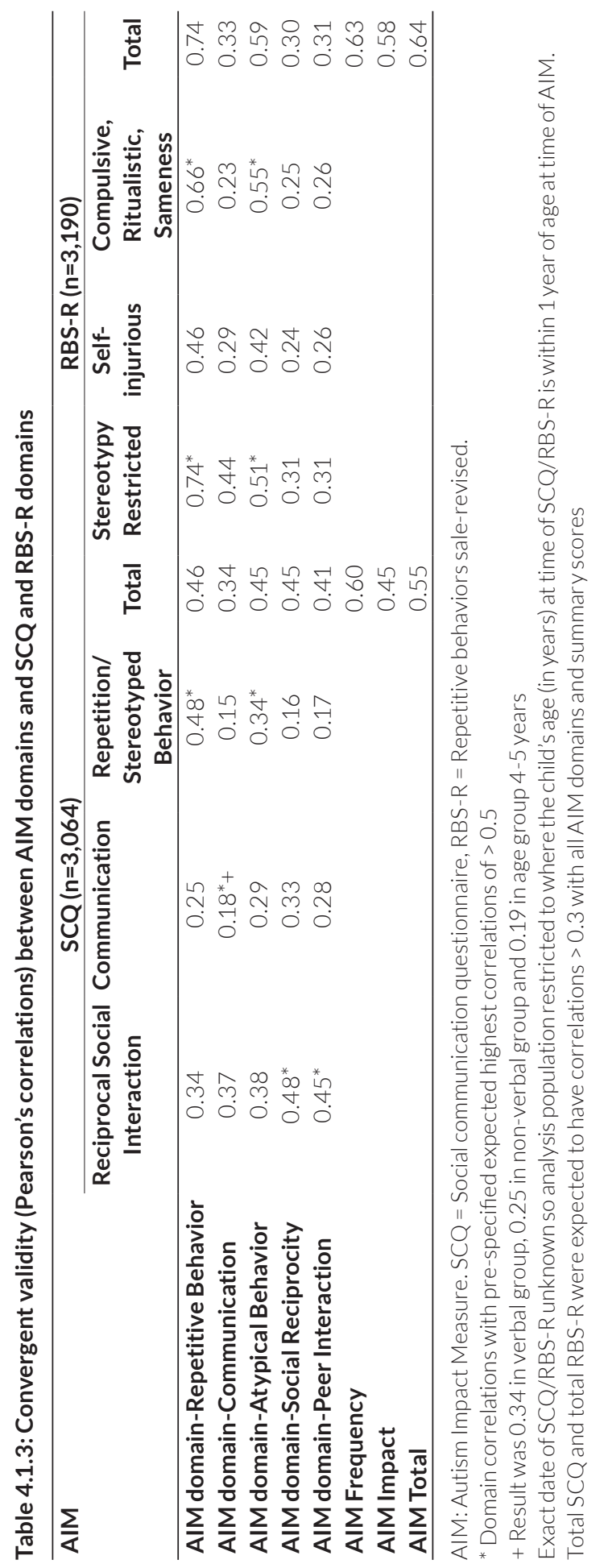




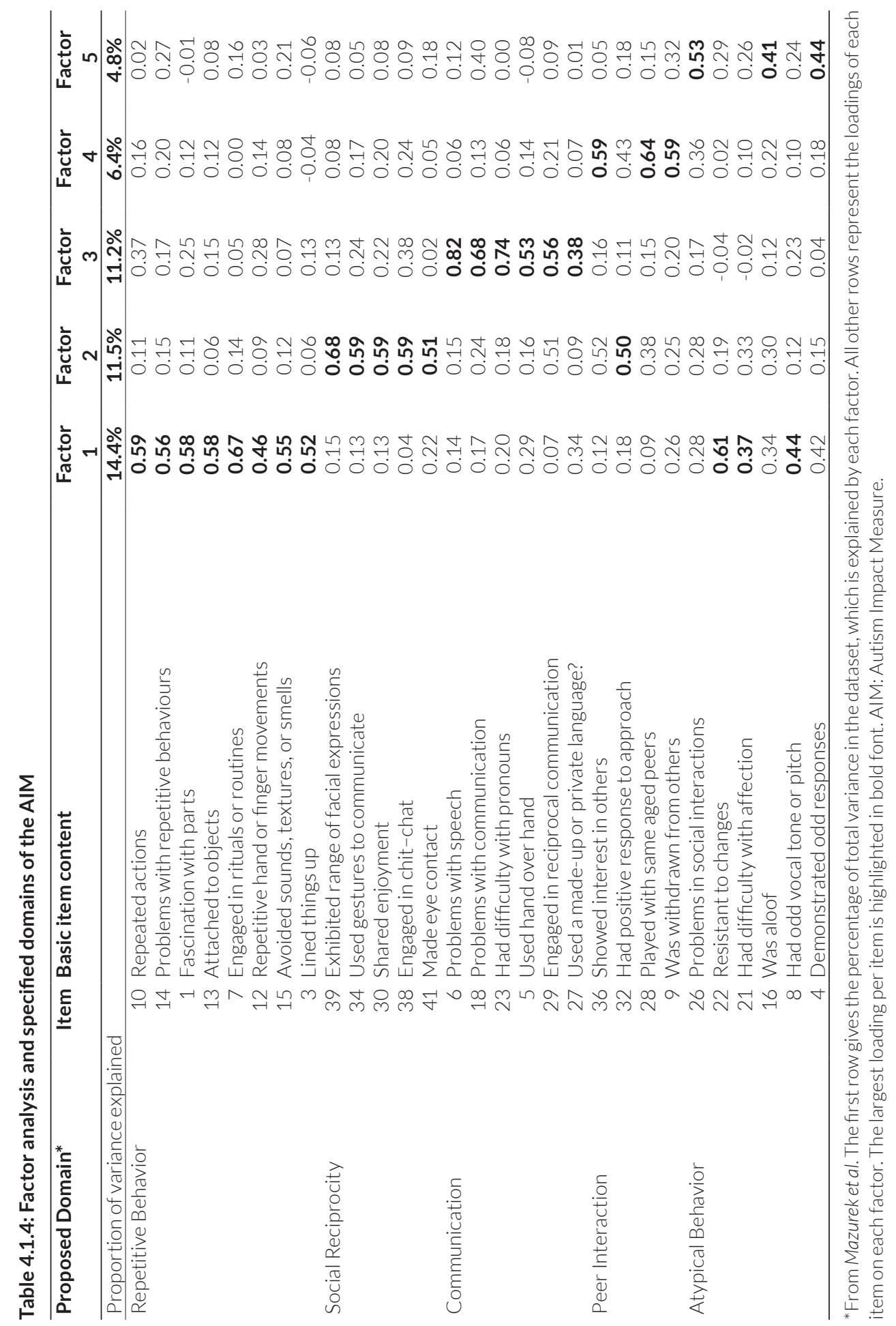




\section{Known-group analysis}

For the patients who completed all items, the mean (median) total AIM score was 220.8 (219). In general, frequency items received higher scores than impact items [119.9 (120) vs 100.9 (99)]. Mean and median scores for the five domains were; Repetitive Behavior: 41.3 (40); Communication: 30.7 (28); Atypical Behavior: 34.8 (35); Social Reciprocity 27.1 (27); Peer Interaction 22.9 (23). All of the above summary scores were approximately normally distributed.

Mean scores for total AIM, frequency, impact and all domains increased monotonically from high IQ to low IQ. These associations of low IQ and greater ASD severity were statistically significant in ANOVA analysis ( $p<0.01$ in all domains). AIM scores were similar between those in full time special education and those who spent less than 30\% of school-time with typically developing peers. Otherwise, AIM scores increased with higher proportion of special-education activity and all differences were statistically significant $(p<0.01)$.

Other "known-groups" were binary-categorised. Both total AIM score (Figure 4.1.2) and impact score (supplement S.4.1.5) were able to differentiate between all pre-defined known-groups ( $p<0.01$ ). All such associations were directionally as expected, with higher scores in the group expected to have more severe ASD. The largest difference in mean total AIM score was between verbal and non-verbal children (257 vs 214, respectively). The frequency score also differentiated between all known groups $(p<0.01)$ except for those children with or without another psychiatric comorbidity ( $p=0.41$, supplement S.4.1.4). Mean scores for the Communication (Figure 4.1.3) and Peer Interaction (supplement S.4.1.9) domains were significantly different $(p<0.01)$ between levels of all 9 pre-defined known-groups. Repetitive Behavior, Social Reciprocity and Atypical Behavior domains significantly $(p<0.01)$ distinguished between levels of 8,8 and 7 of the 9 known-groups respectively, too (see supplements S.4.1.6-S.4.1.8). None of the results for known-groups were altered by adjusting for age, i.e. $p$-values always remained stable (either $\geq 0.05$, between 0.01 and 0.05 , or $<0.01$ ). Results for a total AIM score based on only 29 items were very similar to those based on all 41 items. 
Figure 4.1.2: Mean Total AIM score by known-groups

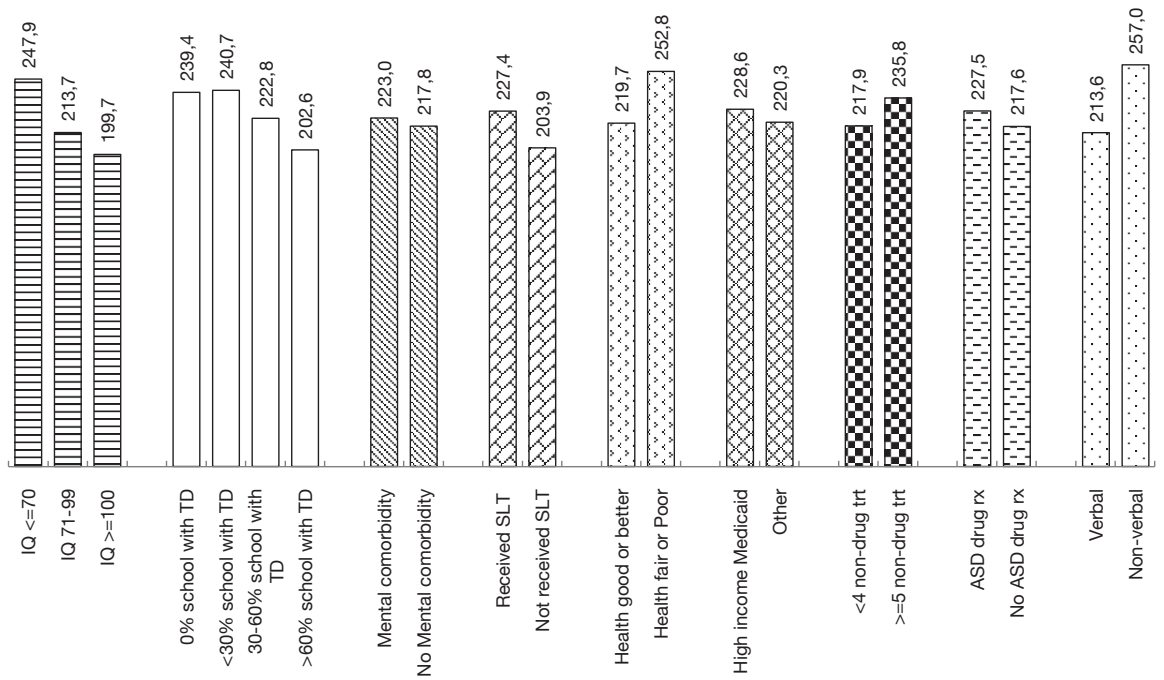

AIM: Autism Impact Measure. TD: typically developing peers, rx: prescription, SLT: Speech and language therapy in last 12 months, trt=treatments, verbal/non-verbal ability as assessed by item 1 of SCQ. Significant differences observed across all known-groups ( $p<0.01$ in both crude analysis and analyses adjusted for age). Higher scores represent higher symptom burden.

Figure 4.1.3: Mean AIM Communication domain score by known-groups

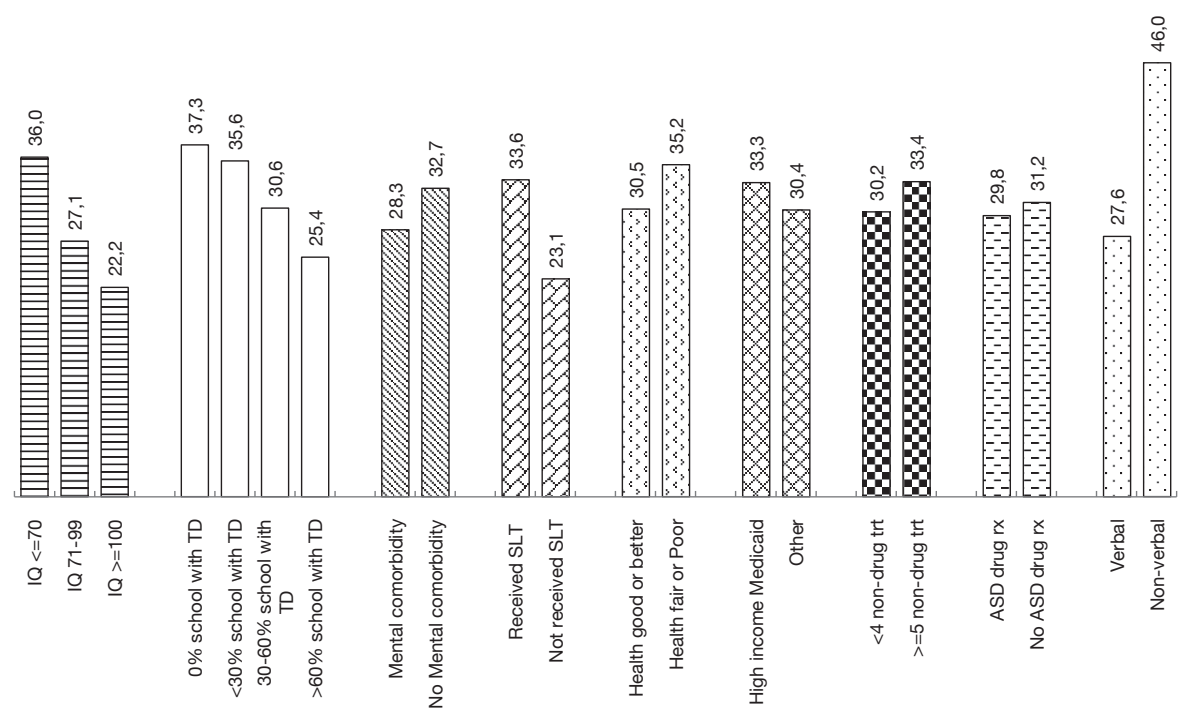

AIM: Autism Impact Measure. TD: typically developing peers, rx: prescription, SLT: Speech and language therapy in last 12 months, trt=treatments, verbal/non-verbal ability as assessed by item 1 of SCQ. Significant differences observed across all known-groups ( $p<0.01$ in both crude analysis and analyses adjusted for age). Higher scores represent higher symptom burden. 


\section{Clinically important response estimates}

For the total AIM score, the CIR estimate ranged from 3.30\% to 8.25\% (Table 4.1.5). This corresponded to a change of between 10.8 and 27.1 points on the raw scale (supplement S.4.1.2). The CIR estimate range for the frequency score was between $3.21 \%$ and $8.04 \%$ and between $3.74 \%$ and $9.34 \%$ for the impact score. Of the domains, Social Reciprocity had the least variability and hence the smallest estimates for the CIR (3.67\% to 9.16\%). All other domains had CIR estimates ranging between $4.20 \%$ and $4.96 \%$ at the lower end, and between $10.49 \%$ and $12.41 \%$ at the upper end.

The largest change in variability across strata was for the Communication domain and IQ level. CIR estimates decreased monotonically from low to high IQ (11.69\% for $1 \mathrm{Q}<70$, 7.78\% for IQ>100; upper estimates). This corresponded to a 3.7 to 5.6 point difference on the raw scale (in which a maximum change of 48 points is possible). This example aside, the data had stable variance across $I Q$ and age ranges, because estimates of variability were generally only slightly higher in the groups with smallest sample size (IQ <70 and age 15-17 years). Generally, variance was slightly smaller within children of similar IQ, rather than of similar age. 


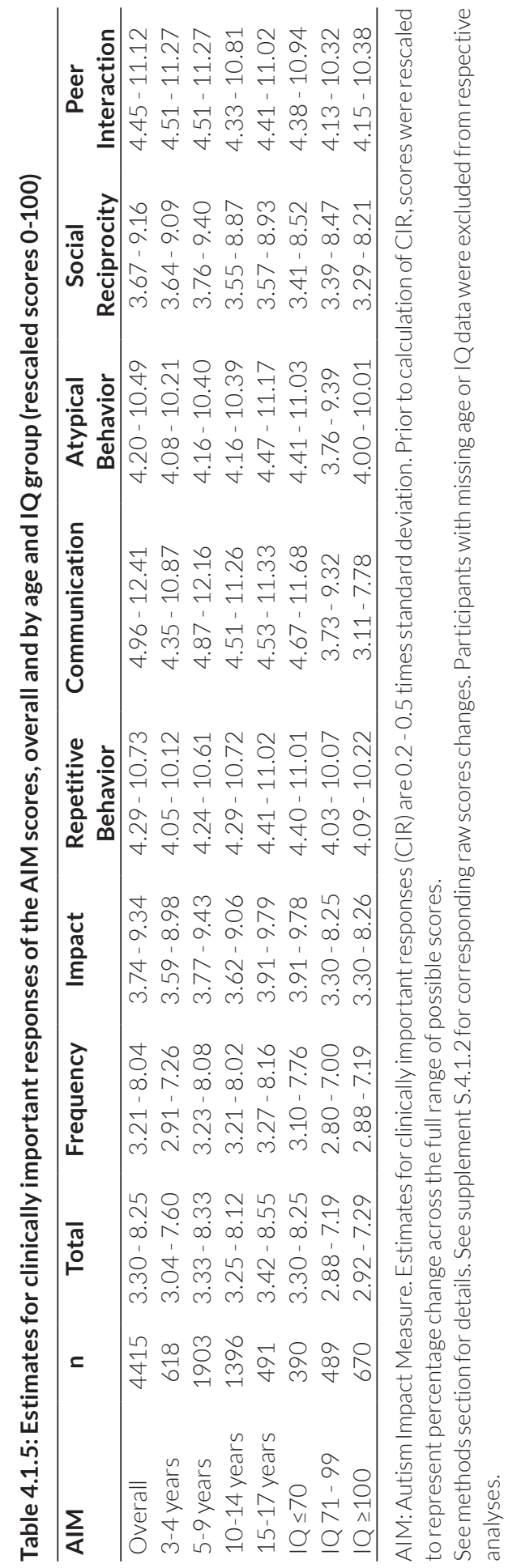




\section{Discussion}

To our knowledge, this study represents the largest fielding of the AIM to an ASD population to date. Our main findings were the estimates of thresholds of clinical importance and the ability of the AIM to separate known groups of children with ASD. We also believe our study represents the first investigations of these concepts for the AIM. Overall, the CIR for the total AIM score was estimated to be in the range of $3.30 \%$ to $8.25 \%$, corresponding to $10.8-27.1$ points of the full 82 to 410 score range. Total AIM scores and most domain scores were generally homogeneous across age and IQ strata, with the only marked exception being that there was more variation in communicative abilities of children with low IQ. Of 9 pre-defined known-groups, the AIM total score statistically differentiated all of them. Mean scores on each of the domains separated almost all known-groups too. Moreover, according to the lower bound for CIR estimates from above, the majority of these differences represented clinical meaningfulness. Even for the Communication domain, which had the largest CIR estimates relative to scale, the lower estimate (5.0\%) was surpassed in all but one of the known-groups (yes/no to current prescription drug for ASD). The more stringent upper estimate of $12.4 \%$ was even achieved in 4 of the 9 known-groups. Namely these groups were: school time with typically developing peers, IQ strata, verbal ability and participation in SLT. In all, these results do provide some confidence that the AIM should be able to respond to symptom changes over time. However, it is uncertain whether any intervention (pharmacological or nonpharmacological) could change such fundamental personal characteristics as represented by our known-groups. Likely our lower estimates for CIR are a most reasonable goal. A limitation of the CIR results is that only distribution-based estimates were generated due to a lack of follow-up data and an appropriate anchor, such as caregiver reported assessment of change. Therefore, further evaluation is required to test empirically the estimates generated.

Our sample, on the whole, was very similar to those used in previous AIM studies ${ }^{1,2}$, in that respondents were mainly mothers of the child with ASD (around 90\%), and families lived at various locations across the US. Children with ASD in each study were mainly male (between 80\%-84\%) and of similar age (between 2-14, 2-16, or 3-17 years). A key difference however, was that we fielded the AIM electronically, rather than on paper. We used this opportunity for secondary objectives of retesting other psychometric properties of the AIM in this format.

Importantly, there were no striking differences in the characteristics of participants or their responses, based on if they used the vertical or horizontal version of the questionnaire. Our data in the most part also confirmed the suitability of an underlying 5 -factor structure of the AIM proposed by Mazurek et al ${ }^{2}$. Items proposed for the Repetitive Behavior, Communication, and Social Reciprocity domains all loaded highly and separately from each other. These first 3 domains accounted for almost $40 \%$ of the 
variability in our data: impressive, given the heterogeneity of symptoms on the autism spectrum. In addition, the magnitude of variability explained by each of these domains was almost equal (11-15\% each) and in line with the three core symptoms of ASD.

External convergent validity of the AIM total scores and most domain scores was also demonstrated. Specifically, both Repetitive Behavior and Atypical Behavior correlated highly ( $r>0.50$ ) with the RBS-R domains of similar concepts. This is despite the RBS-R having no specific recall period, but the AIM having a two-week recall. Correspondence of the AIM to the SCQ total score was also high. Four out of 5 domain-domain relationships that were expected to generate the highest correlation coefficients did exactly that, albeit not to the extent hypothesised ( $r=0.34$ to 0.48). Only the relationship between AIM Communication and SCQ Communication domains were at odds to the expected. The correlation was still positive but of modest magnitude $(r=0.18)$. Sensitivity analysis in children aged 4-5 years - which is the age range asked to focus on for some items of the SCQ ${ }^{10}$ - did not improve this ( $r=0.19$ ). Nonetheless, the AIM Communication domain does represent a clear latent variable, given the perfect representation of this domain mentioned in factor-analysis results above. One explanation is that the AIM and SCQ Communication domains measure subtly different concepts. AIM Communication items mainly already assume verbal ability with some questions relating to concepts like madeup languages, use of pronouns, and reciprocal communication. In contrast some SCQ items relating to communication are specifically omitted for non-verbal children ${ }^{10}$. An alternative explanation is that the AIM directs caregivers to recall symptom severity over the last two weeks, whereas SCQ items have a lifetime perspective. A limitation of this study is that the SCQ and RBS-R surveys were not taken at the same time as the AIM, hence it is difficult to evaluate if non-concordance is due to differences in conceptual constructs or is due to actual differences in symptom severity at time of survey completion. Another more general limitation of the study is that all data are caregiver-reported and therefore some demographic and personal characteristics (e.g. IQ score) may be based on estimates only.

\section{Future research and use of the AIM}

Our CIR estimates above can be used to inform studies wishing to use the AIM in the near future. Better still would be to have repeated follow up in the same patients in order to also estimate CIR based on anchor based approaches ${ }^{22,23}$. This is a possibility, as all data from this current study will be made available via SPARK.

Missing data was slightly more common in the horizontal layout and for impact questions but otherwise was seldom and unsystematic. The most commonly skipped item was only done so by $1.52 \%$ of respondents, but overall we had to exclude around $10 \%$ of the sample, as the developers currently offer no advice on dealing with missing data ${ }^{1,2}$. Given our findings that missing item level data is infrequent, that the AIM has good internal consistency ( $\mathrm{a}=0.96$ ), and that most items are normally distributed, we recommend imputing missing items by multiple imputation (perhaps only excluding some observations 
with missing data above a pre-defined threshold value of e.g. 20\%). This approach has worked well for the AIM elsewhere ${ }^{8}$.

Throughout our analysis, the Frequency and Impact summary scores also displayed good psychometric properties. This means that in addition to total and domain scores, future researchers could use the summed Frequency or Impact scores, depending on their specific question. In particular, the Frequency score might be more useful, because Impact may be more easily affected by other things than interventions, such as coping mechanisms built into everyday life. Furthermore, if an items frequency score is low, then the impact question may become redundant.

Electronically reported outcome measures have added benefits over paper-based measures. These include the avoidance of data entry errors, increased willingness of respondents to share sensitive information, and quicker access to this data for research ${ }^{24}$. Electronic measures can also be completed remotely. A clear advantage of the AIM, is the limited time needed to complete it (median time: 7 minutes). This coupled with high overall participation rate in our study ${ }^{8}$ demonstrates that caregivers are comfortable completing the AIM in such a way. This means that the AIM could potentially enable cheaper and low burden monitoring of severity changes as well as effectiveness of interventions in a real-world setting.

\section{Conclusion}

Our study provides estimates of thresholds of clinical importance for the AIM, as well as some indication that the AIM can distinguish between known groups of children with ASD. Our results also confirm the validity of the AIM based on other important psychometric properties. When administered electronically, the AIM offers a quick and relatively inexpensive method for caregivers to report core symptoms of children with ASD, including communication deficits, difficulties with social interactions and repetitive behaviours. 


\section{References}

1. Kanne, S. M. et al. The Autism Impact Measure (AIM): initial development of a new tool for treatment outcome measurement. J. Autism Dev. Disord. 44, 168-179 (2014).

2. Mazurek, M. O. et al. Construct Validity of the Autism Impact Measure (AIM). J. Autism Dev. Disord. 50, 2307-2319 (2020).

3. Lord, C.et al. Autism diagnostic observation schedule, second edition. (Western Psychological Services, 2012).

4. Barthélémy, C.etal.Validation of the Revised Behavior Summarized Evaluation Scale. J. Autism Dev. Disord. 27, 139-153 (1997).

5. Freeman, B. J., Ritvo, E. R., Yokota, A. \& Ritvo, A. A Scale for Rating Symptoms of Patients with the Syndrome of Autism in Real Life Settings. J. Am. Acad. Child Psychiatry 25, 130-136 (1986).

6. Constantino, J. N. \& Gruber, C. P. Social responsiveness scale (SRS). (Western Psychological Services., 2012).

7. SPARK Consortium.SPARK:A US Cohort of 50,000 Families to Accelerate Autism Research. Neuron 97, 488-493 (2018).

8. Monz, B., Houghton, R., Law, K. \& Loss, G. Treatment patterns in children with autism in the United States. Autism Res. 12, 517-526 (2019).

9. Chandler, S. et al. Validation of the social communication questionnaire in a population cohort of children with autism spectrum disorders. J. Am. Acad. Child Adolesc. Psychiatry 46, 1324-1332 (2007).

10. Rutter, M., Bailey, A., Berument, S. K., Le Couteur, A. \& Lord, C. Social communication questionnaire (SCQ) manual. (Western Psychological Services, 2003).

11. Mirenda, P.et al. Validating the Repetitive Behavior Scale-revised in young children with autism spectrum disorder. J. Autism Dev. Disord. 40, 1521-1530 (2010).

12. Cronbach, L. J. Coefficient alpha and the internal structure of tests. Psychometrika 16, 297-334 (1951).

13. Nunnally, J. C. \& Bernstein, I. H. Psychometric theory (third ed.).ISBN: 9780070478497 (McGraw-Hill Companies, 1994).
14. Kanne, S. M. et al. The Role of Adaptive Behavior in Autism Spectrum Disorders: Implications for Functional Outcome. J. Autism Dev. Disord. 41, 1007-1018 (2011).

15. Mayes, S. \& Calhoun, S. Impact of IQ, age, SES, gender, and race on autistic symptoms. Res. Autism Spectr. Disord. 5, 749-757 (2011).

16. Rosen, T., E., Spaulding, C., J., Gates, J., A. \& Lerner, M., D. Autism severity, co-occurring psychopathology, and intellectual functioning predict supportive school services for youth with autism spectrum disorder. Autism. 23, 1805-1816 (2019).

17. Spaulding, C., J., Lerner, M., D. \& Gadow, K. Trajectories and correlates of special education supports for youth with autism spectrum disorder and psychiatric comparisons. Autism 21, 423-435 (2017).

18. Rosenberg, R. E., Kaufmann, W., E., Law, K., J. \& Law, P. A. Parent Report of Community Psychiatric Comorbid Diagnoses in Autism Spectrum Disorders. Autism Res. Treat. 2011:405849, 1-10 (2011).

19. Coon, C. D.\& Cappelleri, J. C. Interpreting Change in Scores on Patient-Reported Outcome Instruments. Ther. Innov. Regul. Sci. 50, 22-29 (2016).

20. Fayers, P. M. \& Hays, R. D. Don't middle your MIDs: regression to the mean shrinks estimates of minimally important differences. Qual. Life Res.23, 1-4 (2014).

21. Norman, G. R., Sloan, J. A. \& Wyrwich, K. $W$. Interpretation of changes in healthrelated quality of life: the remarkable universality of half a standard deviation. Med. Care 41, 582-592 (2003).

22. Engel, L., Beaton, D. E. \& Touma, Z. Minimal Clinically Important Difference: A Review of Outcome Measure Score Interpretation. Rheum. Dis. Clin. North Am. 44, 177-188 (2018).

23. Wright, A., Hannon, J., Hegedus, E. J. \& Kavchak, A. E. Clinimetrics corner: a closer look at the minimal clinically important difference (MCID). J. Man. Manip. Ther. 20, 160-166 (2012).

24. Deshpande, P. R., Rajan, S., Sudeepthi, B. L. \& Abdul Nazir, C. P. Patient-reported outcomes: A new era in clinical research. Perspect. Clin. Res. 2, 137-144 (2011). 


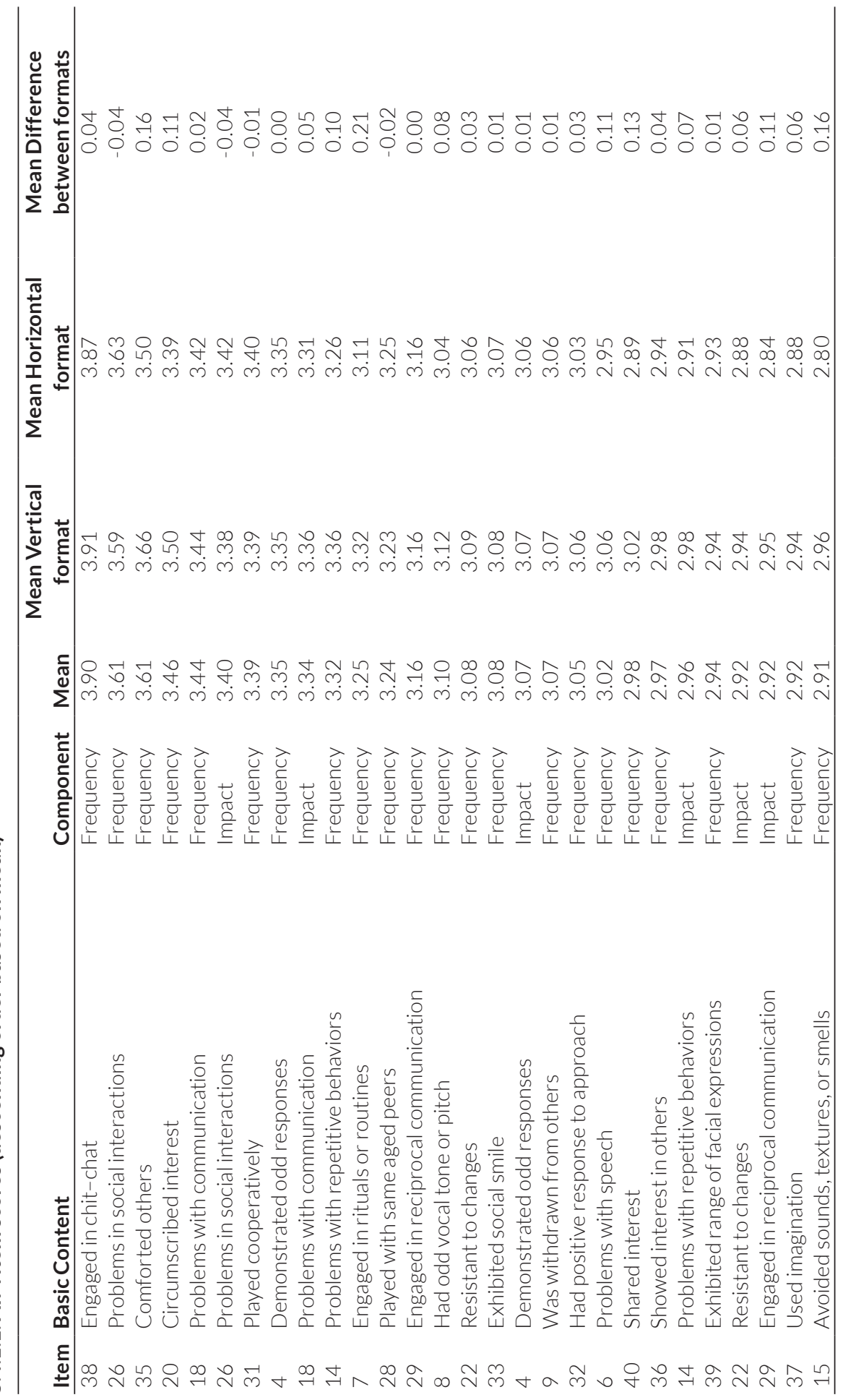




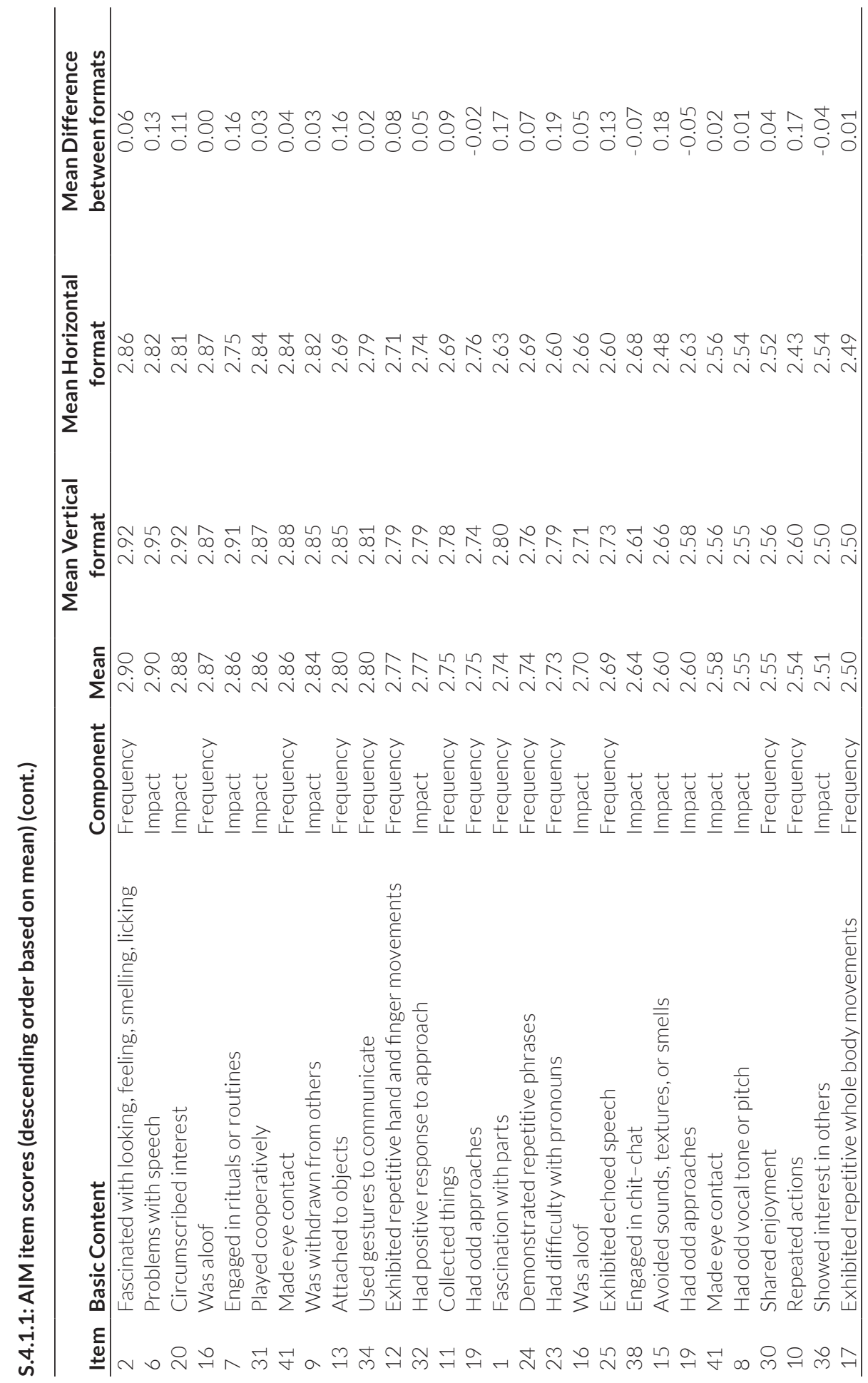




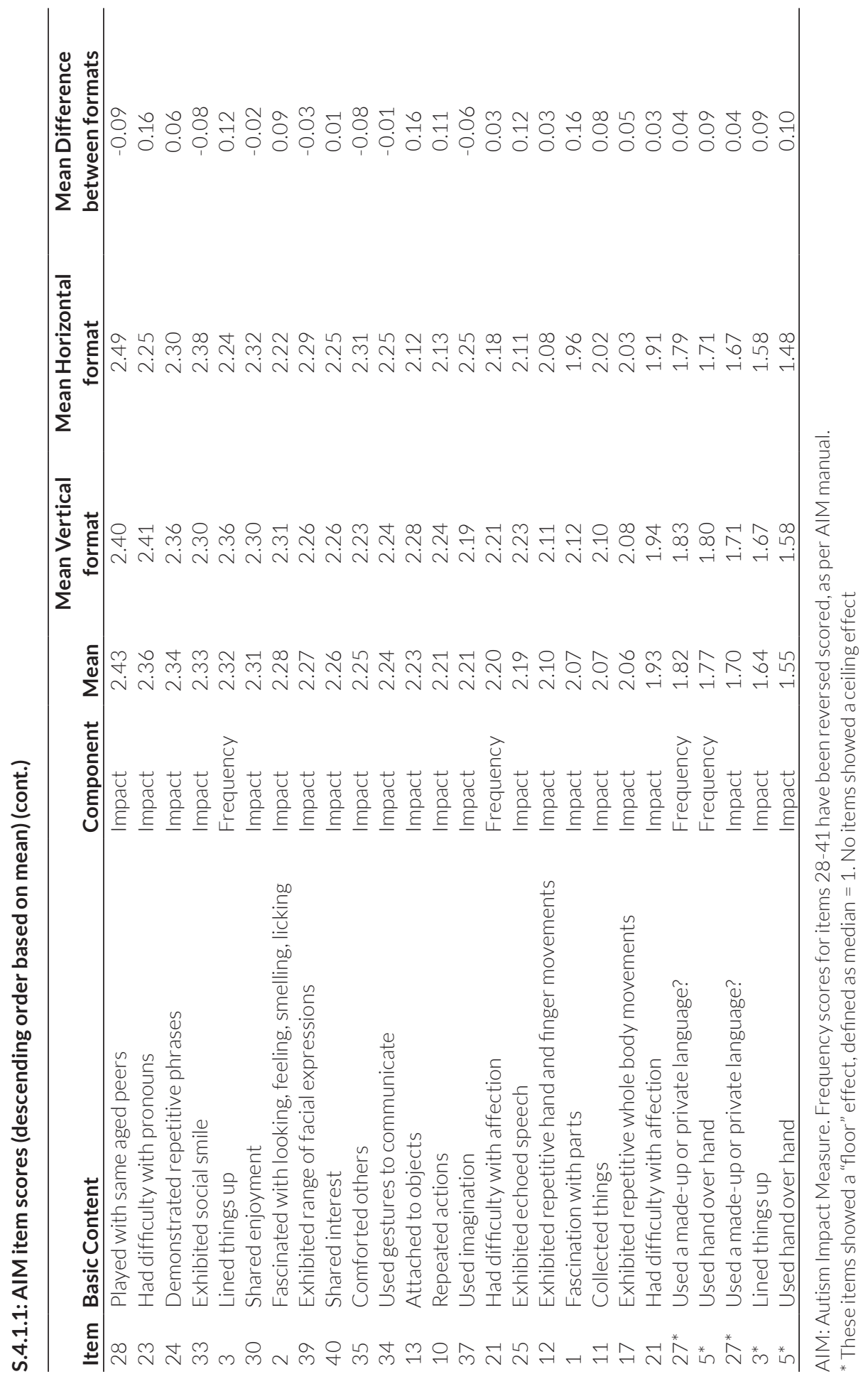




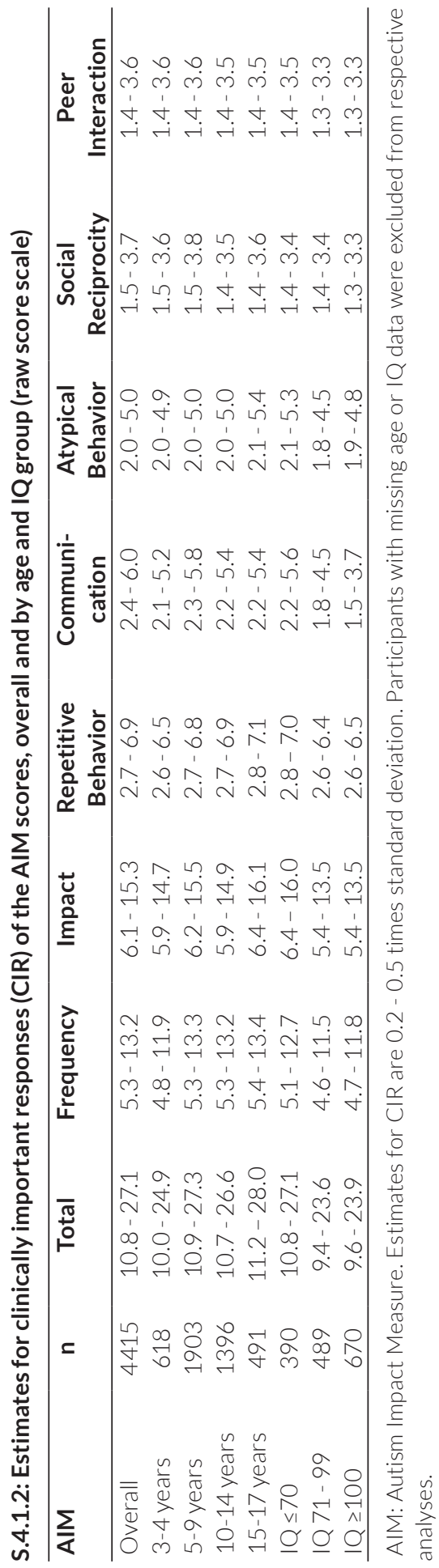




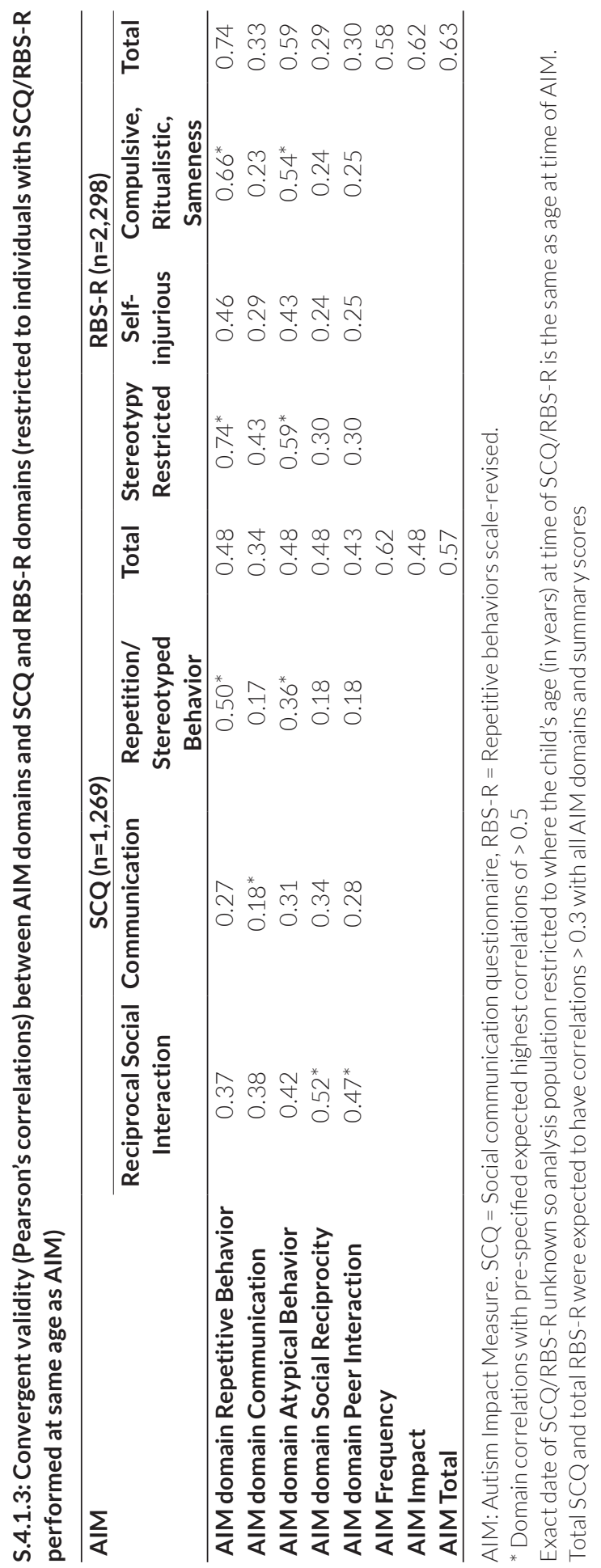




\section{S.4.1.4: Mean AIM Frequency score by known-groups}

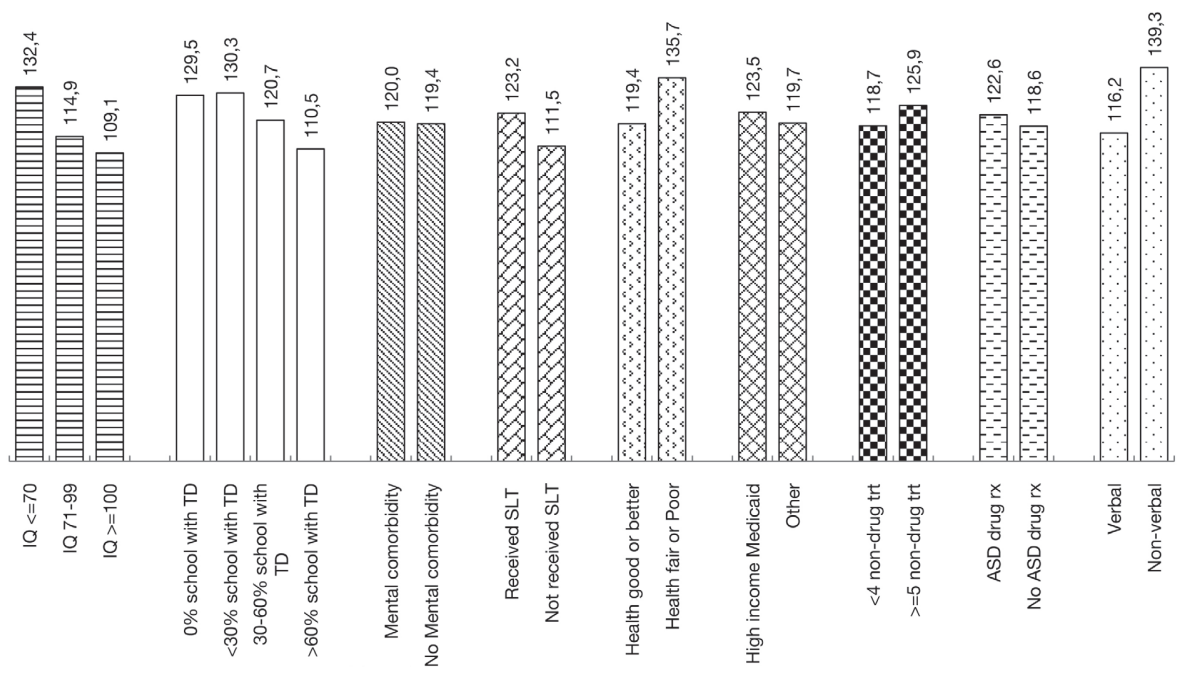

AIM: Autism Impact Measure. TD: typically developing peers, rx: prescription, SLT: Speech and language therapy in last 12 months, trt=treatments, verbal/non-verbal ability as assessed by item 1 of SCQ. Significant differences observed across all known-groups $(p<0.01$ in both crude analysis and analyses adjusted for age), except for mental comorbidity vs no mental comorbidity ( $p=0.41$, adjusted $p=0.42$ ). Higher scores represent higher symptom burden.

\section{S.4.1.5: Mean AIM Impact score by known-groups}

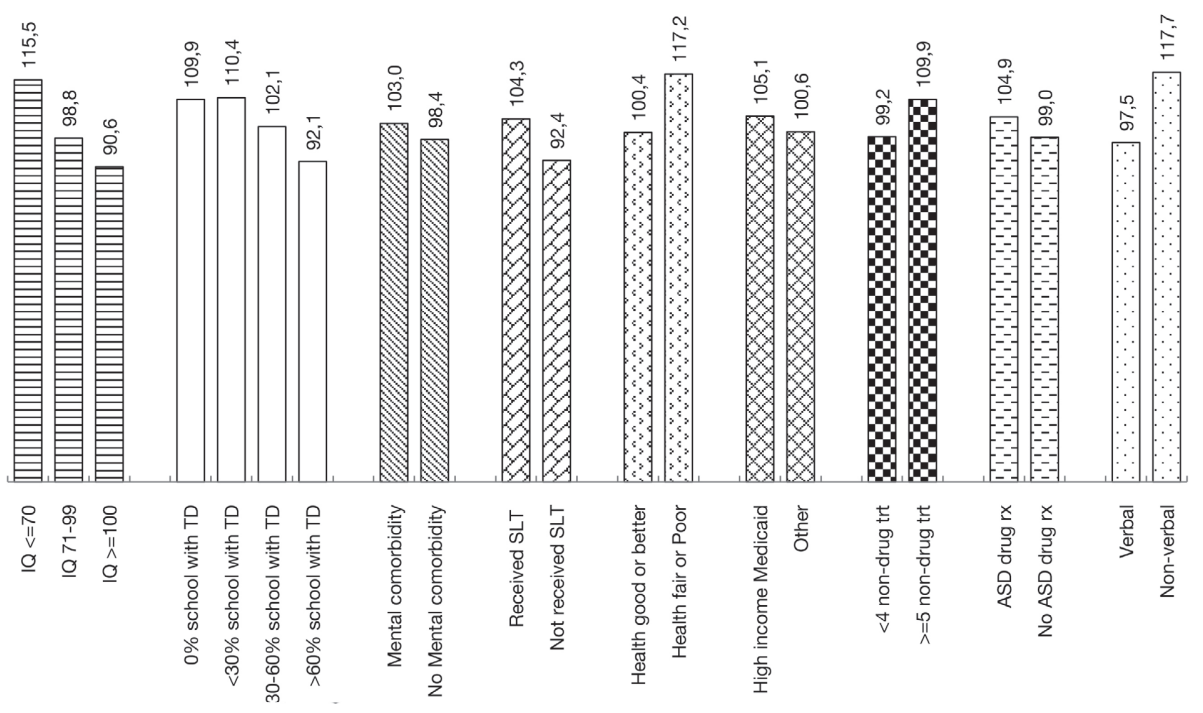

AIM: Autism Impact Measure. TD: typically developing peers, rx: prescription, SLT: Speech and language therapy in last 12 months, trt=treatments, verbal/non-verbal ability as assessed by item 1 of SCQ. Significant differences observed across all known-groups ( $p<0.01$ in both crude analysis and analyses adjusted for age). Higher scores represent higher symptom burden. 


\section{S.4.1.6: Mean AIM Repetitive Behavior score by known-groups}

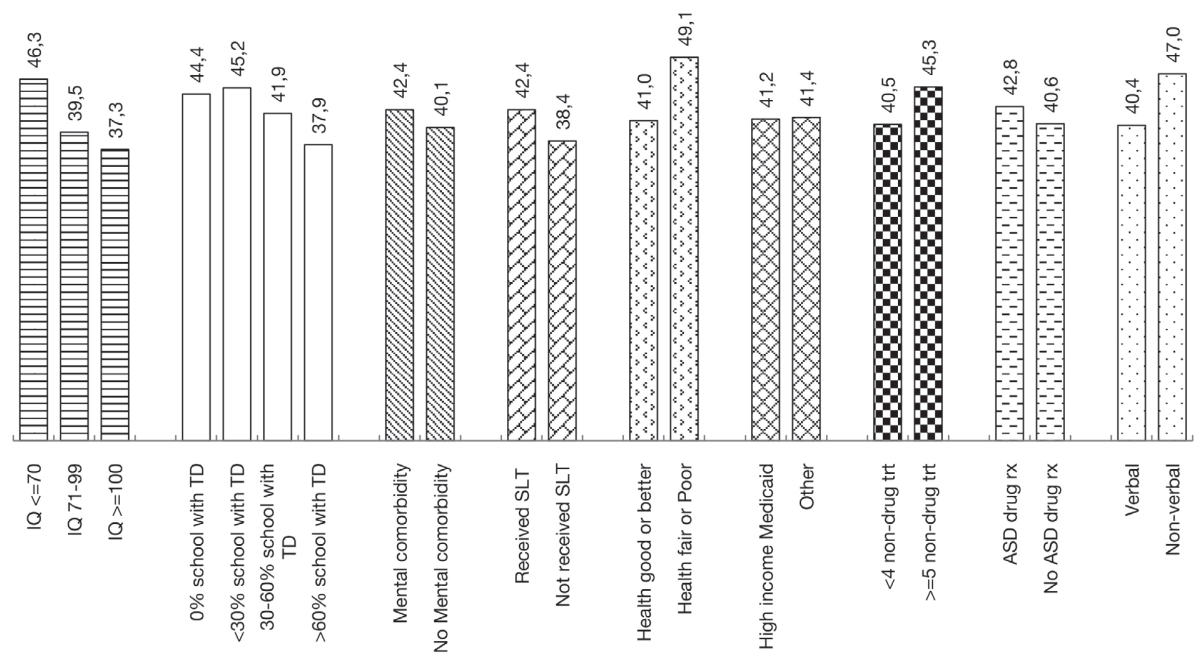

AIM: Autism Impact Measure. TD: typically developing peers, rx: prescription, SLT: Speech and language therapy in last 12 months, trt=treatments, verbal/non-verbal ability as assessed by item 1 of SCQ. Significant differences observed across all known-groups $(p<0.01$ in both crude analysis and analyses adjusted for age), except for High income Medicaid vs other $(p=0.77$, adjusted $p=0.77)$. Higher scores represent higher symptom burden.

\section{S.4.1.7: Mean AIM Atypical Behavior score by known-groups}

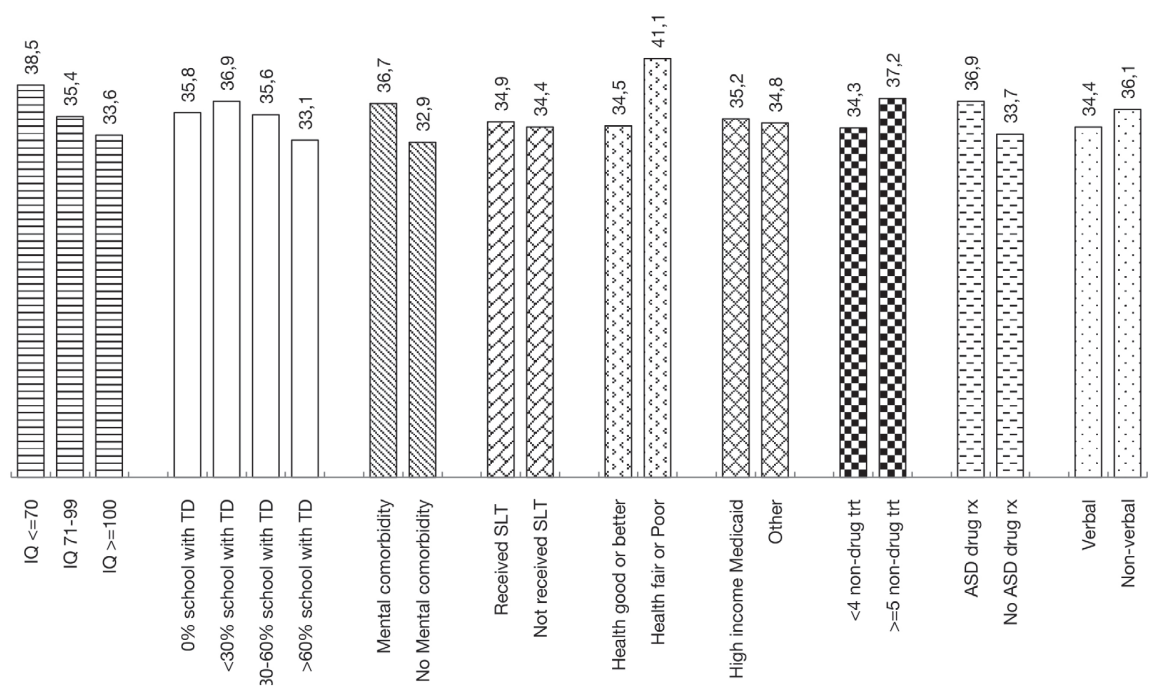

AIM: Autism Impact Measure. TD: typically developing peers, rx: prescription, SLT: Speech and language therapy in last 12 months, trt=treatments, verbal/non-verbal ability as assessed by item 1 of SCQ. Significant differences observed across all known-groups ( $p<0.01$ in both crude analysis and analyses adjusted for age), except for SLT vs no SLT ( $p=0.17$, adjusted $p=0.16$ ) and high income Medicaid vs other $(p=0.40$, adjusted $p=0.40$ ). Higher scores represent higher symptom burden. 


\section{S.4.1.8: Mean AIM Social Reciprocity score by known-groups}

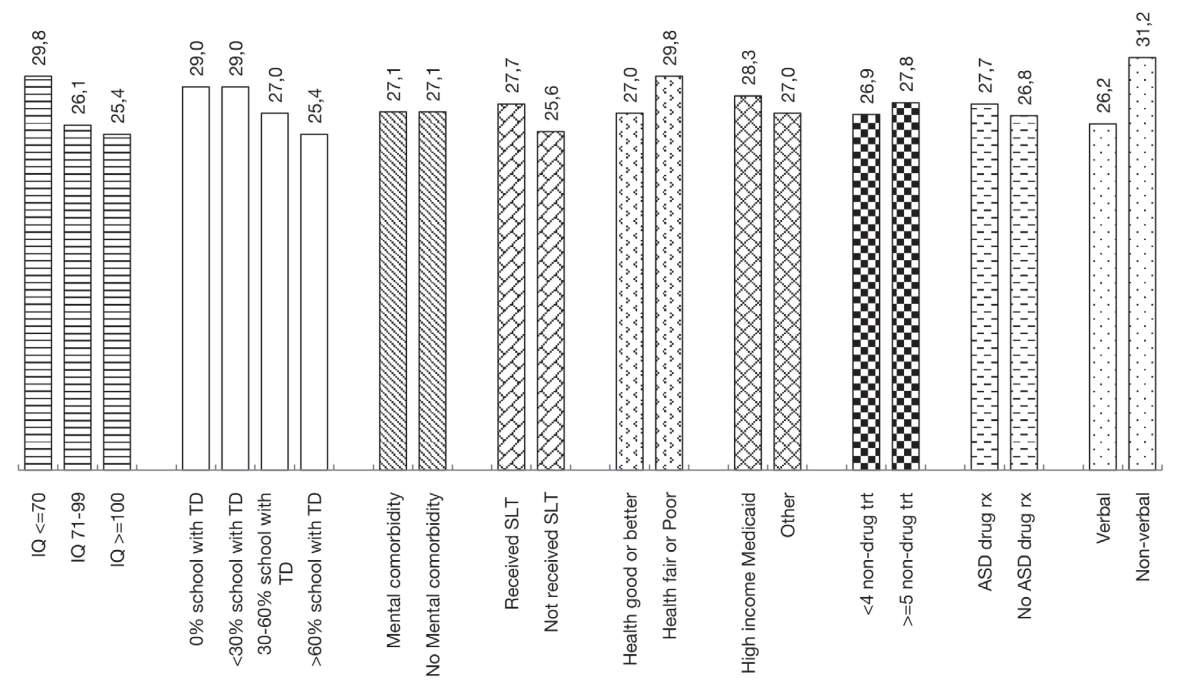

AIM: Autism Impact Measure. TD: typically developing peers, rx: prescription, SLT: Speech and language therapy in last 12 months, trt=treatments, verbal/non-verbal ability as assessed by item 1 of SCQ. Significant differences observed across all known-groups ( $p<0.01$ in both crude analysis and analyses adjusted for age), except for mental comorbidity vs no mental comorbidity ( $p=0.97$, adjusted $p=0.98$ ).

\section{S.4.1.9: Mean AIM Peer Interaction score by known-groups}

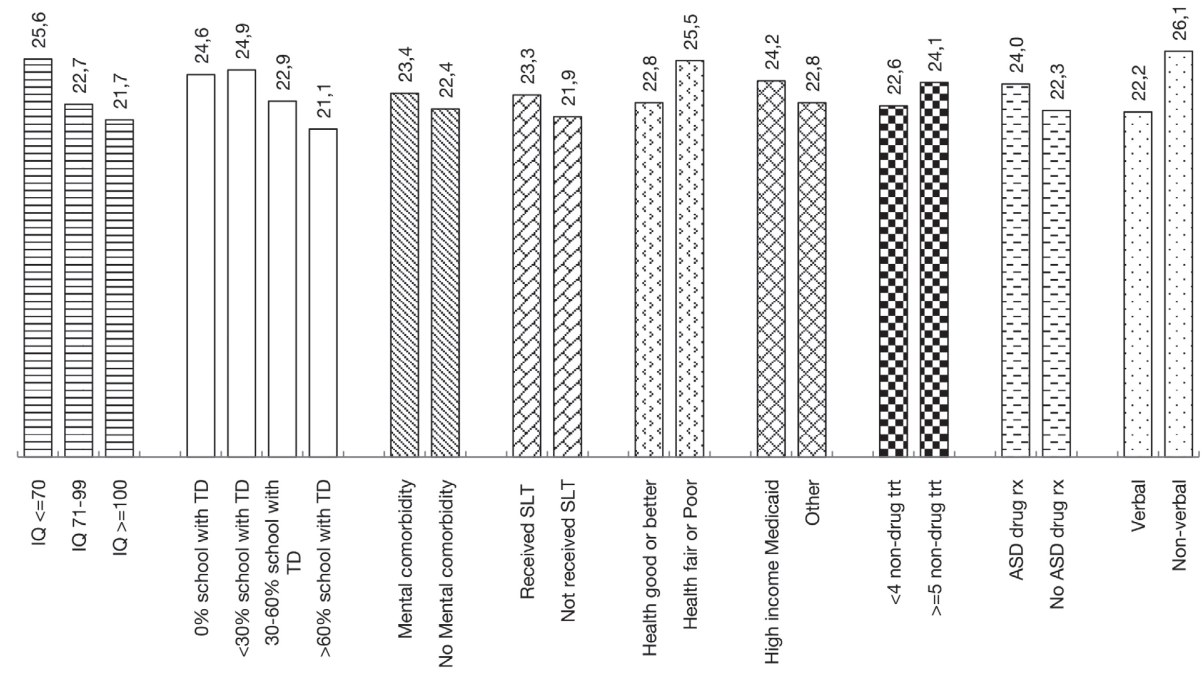

AIM: Autism Impact Measure. TD: typically developing peers, rx: prescription, SLT: Speech and language therapy in last 12 months, trt=treatments, verbal/non-verbal ability as assessed by item 1 of SCQ. Significant differences observed across all known-groups ( $p<0.01$ in both crude analysis and analyses adjusted for age). 



\section{Chapter 5}

General discussion 
This thesis explored utilisation and outcomes of treatment in ASD. Chapter 1 set out the background to this work, by characterising the current ASD treatment landscape and some important knowledge gaps. In Chapter 2, we provided up to date estimates for psychotropic drug ("drug") and non-drug treatment use in ASD, expanding current literature in terms of age range studied (adults as well as children), geographies (USA and UK data), comparing to non-ASD comparator groups, and examining predictors of use. For non-drug treatments, we specifically assessed the association of geography and health insurance type with their uptake. In Chapter 3, we considered potential unintended effects of commonly used drugs in children with ASD: firstly, a possible relationship between ADHD medications and serious cardiovascular events (SCV), and secondly, a comparison of risperidone versus aripiprazole for differences in bone fracture risk, over the short and long term. In Chapter 4 we validated a new outcome measure for ASD symptom severity.

In this final chapter, we summarise and discuss the main findings, address important methodological considerations encountered, and reflect on main implications and recommendations for policy and future research.

\section{Summary of findings in context}

The aim of Chapter 2 was to describe the types and prevalence of drug and non-drug treatment use in ASD. For drug treatments, specific sub-goals were to extend previous literature by studying adults (as well as children), assess a non-USA cohort (UK as well as the USA) and to compare ASD to non-ASD groups. We also provided more up to date estimates than previous studies. For non-drug use, the novel aspect was to evaluate a USA cohort, as data was previously lacking in the area. We also explored possible predictors of treatments, and hypothesised specifically about the impact of geography (urbanisation level) and insurance type on uptake rates of non-drug treatments. Table 5.1 summarises the overall levels of treatment use found in this thesis, by country, insurance type and age group.

\section{Drug treatment}

The overall results demonstrated high levels of psychotropic treatment use in ASD, and especially so in the USA. Previous USA based claims-data studies in children found annual rates of any psychotropic treatment in the range of 56-65\% ${ }^{1-4}$, which encompasses our estimates of 59-65\% in Chapter 2.1. A comparable study ${ }^{5}$ in Medicaid adults with ASD found $85 \%$ use versus our estimate of $81 \%$. Polypharmacy (defined as two or more treatments with 30 or more overlapping prescription days) in children with ASD was previously reported at 33-35\% 3,4 versus our estimate of 31-40\% (no comparable historical data in adults). In the USA, the main difference between this thesis and the previous studies is that the previous studies were based on data between 2000 and 2009 , whereas our data were from 2014. 
Table 5.1: percentage of ASD patients receiving drug and non-drug treatments annually, by country, insurance type and age group

\begin{tabular}{|c|c|c|c|c|c|c|c|c|c|c|c|}
\hline & \multirow[t]{2}{*}{$\begin{array}{l}\text { Country and } \\
\text { insurance type }\end{array}$} & \multirow[t]{2}{*}{$\begin{array}{c}\text { Calendar } \\
\text { year }\end{array}$} & \multicolumn{6}{|c|}{ Age group (years) } & \multicolumn{3}{|c|}{$\begin{array}{l}\text { Weighted averages } \\
\text { (age in years) }\end{array}$} \\
\hline & & & $3-4$ & $5-11$ & $12-17$ & $18-24$ & $25-49$ & $50+$ & $\begin{array}{l}\text { Child } \\
(3-17)\end{array}$ & $\begin{array}{c}\text { Adult } \\
\text { (18 and } \\
\text { older) }\end{array}$ & $\begin{array}{c}\text { Total } \\
\text { ( } 3 \text { and } \\
\text { older) }\end{array}$ \\
\hline \multirow[t]{3}{*}{ Drug } & $\begin{array}{l}\text { USA Medicaid } \\
\text { (Chapter 2.1) }\end{array}$ & 2014 & 25 & 63 & 80 & 81 & 82 & 83 & 65 & 81 & 69 \\
\hline & $\begin{array}{l}\text { USA commercial } \\
\text { (Chapter 2.1) }\end{array}$ & 2014 & 11 & 52 & 76 & 81 & 72 & 69 & 59 & 78 & 64 \\
\hline & $\begin{array}{l}\text { UK NHS } \\
\text { (Chapter 2.2) }\end{array}$ & 2015 & 11 & 21 & 26 & 35 & 51 & 70 & 24 & 44 & 32 \\
\hline \multirow[t]{3}{*}{$\begin{array}{l}\text { Non- } \\
\text { drug }\end{array}$} & $\begin{array}{l}\text { USA Medicaid } \\
\text { (Chapter 2.3) }\end{array}$ & $2016 / 17$ & $100^{*}$ & 97 & 93 & - & - & - & 96 & - & - \\
\hline & $\begin{array}{l}\text { USA commercial } \\
\text { (Chapter 2.3) }\end{array}$ & 2016/17 & 98 & 97 & 92 & - & - & - & 95 & - & - \\
\hline & $\begin{array}{l}\text { Europe / [UK] } \\
\left(\text { Salomone et al) }{ }^{9}\right.\end{array}$ & 2012 & 91 & {$[75]$} & - & - & - & - & $\begin{array}{l}91 / \\
{[75]}\end{array}$ & - & - \\
\hline
\end{tabular}

Numbers in each cell represent the proportion of patients that received treatment annually from within each age group.

* Rounded; 206/207 received a non-drug treatment.

NHS=National Health Service. USA=United States of America, UK=United Kingdom.

Numbers for USA non-drug treatments are reported in different age groups in this table than in Chapter 2.3 to aid comparisons to other chapters. Results from Salomone et al included for comparison purposes due to similar study design in Chapter 2.3 but only includes children aged 1-6 years; results are the mean score across 18 European countries [UK results in brackets].

In the UK, a previous study in ages up to 24 years found any psychotropic treatment use and polypharmacy at approximately 29\% and 7\% respectively, between 1999 and $2008{ }^{6}$. In Chapter 2.2, for year 2015, we found 27\% use for any drug and 7\% for polypharmacy (weighted average across the same age range). The previous UK study ${ }^{6}$ defined polypharmacy as two or more treatments in a calendar year, whereas we defined polypharmacy as two or more treatments overlapping for at least 30 days. Another recently published UK study found 33\% use across all age groups between 2009 and $2016^{7}$ which compares to our estimate of $32 \%$.

Our results indicate little change in prevalence of exposure to psychotropic treatment use, in both the UK and the USA over recent years. A recent systematic review ${ }^{8}$ reported median (not weighted mean) estimates of any psychotropic use at $42 \%$ in children and $62 \%$ in adults: but these were less useful for comparative purposes as they included a mix of countries (mainly North America), a mix of observation years (spanning over 30 years) and many smaller survey studies too. 
Our results demonstrated substantially more psychotropic treatment use in the USA than in the UK. For adults, the proportion of patients receiving treatment were around twice as high (44\% vs $78-81 \%$ ). For children, this ratio was even higher (24\% vs $59-65 \%$ ). In the UK, treatment rates increased gradually throughout childhood and adulthood years, with small increases in treated proportions at each increasing age group. In contrast, in the USA, peak treatment rates were already reached by teenage years (age group 12-17 years), and plateaued thereafter. This demonstrates a tendency to use drug treatments earlier in life for people with ASD in the USA too.

There are various reasons why prescription rates in the UK may be lower than in the USA. Firstly, there are differences between countries in terms of approved treatments and guidelines. As summarised in Chapter 1, in the USA, the antipsychotics risperidone and aripiprazole are the only approved for medications for irritability and aggression in children with ASD, whereas in Europe there are no medications with specific mention of autism in the list of approved indications. In the UK specifically, treatment guidelines published by the National Institute for Health and Care Excellence (NICE) do reflect this, but nonetheless also recommend that antipsychotics be considered for managing "behaviour that challenges" in ASD children and adults when psychosocial interventions have not helped or cannot be adhered to ${ }^{10,11}$. The guidelines also theoretically accommodate use of other medications frequently prescribed in the USA too, so long as they are for comorbid conditions and not ASD itself. For example, provided proper benefit-risk considerations, the selective serotonin re-uptake inhibitor (SSRI) fluoxetine can be recommended as part of first-line care for moderate to severe depression in children and adults ${ }^{12,13}$. Stimulant medication can be recommended for ADHD if symptoms persist after making environmental modifications ${ }^{14}$. NICE guidelines generally recommend psychotropic treatments are started by paediatric or psychiatry specialists however 10,11,14. Indeed, we found that people with ASD in the UK still received more drug treatments than the general non-ASD population (32\% vs 7\%) of similar age, sex and region. Of note however was that only $14 \%$ of UK ASD patients without a documented psychiatric comorbidity were prescribed a psychotropic drug. This compares to around one-third (31-33\%) of USA patients with no documented psychiatric comorbidity that still received a drug prescription - despite similar guidance to only use psychotropic drugs for specific target symptoms or comorbidities in the USA ${ }^{15}$. In summary, differences in approvals and guidelines likely play a role in differences between USA and UK prescribing rates, but do not explain differences entirely.

A second possible contributing factor is differences in healthcare systems. In the UK, most referrals for psychiatric services, like all other non-emergency care, are made via an initial consultation with the patient's general practitioner (GP). This is a routine part of the "gatekeeper" role of GPs in the UK National Health Service (NHS). A recent survey among UK GPS however found widespread lack of clarity for referral pathways specific to ASD ${ }^{16}$. They also reported a general lack of ASD training and were least confident about 
knowing which medications to prescribe. The lack of knowledge about ASD in primary care is also perceived by caregivers and patients themselves, who report being less likely to even seek care due to poor experiences with non-specialists ${ }^{17,18}$. In the USA, similar difficulties occur for ASD patients in the primary care setting ${ }^{19-21}$, but not all patients necessarily need to consult a primary care physician before self-referral to specialists, especially those on non-capitated health plans (i.e. health plans that operate on a feefor-service basis; not with a flat fee for every patient covered). This corroborates the finding in Chapter 2.1 that an independent predictor of treatment use in the USA was being in a non-capitated health plan. While capitated plans can control healthcare costs, there is no clear evidence if they result in comparable outcomes ${ }^{22}$. A related issue on the potential underreporting of psychotropic drugs in the CPRD database is discussed in the methodological considerations section of this chapter.

Thirdly, differences in physician and patient attitudes towards drug prescribing may play a role. There appears to have been little direct market research published on this topic, but there are other available indicators of similarities and differences between countries. First, the proportion of all ASD research funding committed to finding treatments has been comparable between the USA and UK in recent years (approximately $18 \%$ in the UK ${ }^{23}$ and $19 \%$ in the USA ${ }^{24}$ ). Second, perceived-stigma and self-stigma around mental health has been declining in both countries ${ }^{25-27}$ which makes help-seeking more likely ${ }^{28}$. A third aspect is the ongoing debate reaching national newspapers in both countries concerning if autism really is a "disability" to be treated or a "difference" to be accepted ${ }^{29-31}$. While the debate is mainly centred around the appropriate emphasis to be placed on finding a "cure" for autism, it is not unreasonable to imagine that public opinion on this topic could play a role in the decision to seek out or prescribe available treatments for non-core ASD symptoms and comorbidities too. Public and physician awareness campaigns to ensure appropriate use of antipsychotic medication have been launched in the USA ${ }^{32,33}$. Since 2016 in the UK, a national program to stop "over medication" of all psychotropic drugs to people with ASD or intellectual disabilities, has been supported by NHS England ${ }^{34}$. Other differences include historically higher rates of psychotropic drug use in the USA, despite increases in recent years in Europe ${ }^{35-37}$. And finally, direct to consumer advertising of prescription drugs in the USA has been shown to play a role in increased prescription rates for other psychiatric indications ${ }^{38,39}$, whereas this practice is illegal in the UK, in line with patient and physician beliefs ${ }^{40,41}$.

Other main findings were that in all age groups, the Medicaid cohort had slightly higher use of psychotropic drugs than patients with private insurance, perhaps reflecting a higher prevalence of comorbidities and severity of symptoms that made these individuals eligible for Medicaid in the first place. We also found that foster care and White race were associated with higher treatment rates. Conscious and unconscious biases that limit adequate diagnosis and treatment of other psychiatric conditions for Black and ethnic minority youth in the USA are well documented ${ }^{42-45}$. In the UK, as per previous EMR studies ${ }^{6,7}$, 
females were more likely to be prescribed medications than males, but we found no difference between genders in the USA.

\section{Non-drug treatments}

In Chapter 2.3, we evaluated the annual rate of non-drug treatments in ASD children in the USA. The study design was a cross-sectional web-based survey of over 5,122 caregivers. Caregivers reported that almost all children (96\%) received a non-drug treatment in the past 12 months, with speech and language therapy (SLT; 71\%), occupational therapy (OT; 60\%) and behavioural therapy (56\%) being the most common. SLT and OT were more often provided in school while behavioural therapy and psychological interventions were more frequently provided outside school. Psychological therapy and a catch-all category of "other" therapies (such as academic support, recreational and/or animal-based therapy) were the only types of treatment to increase with age.

In a similar survey study in 18 countries in Europe by Salomone et al ${ }^{9}$, a high rate of any non-drug treatment use was observed too (91\%). Only 75\% of children received these services in the UK however, and authors suggested the variation by country may reflect that some respondents did not report treatments that were provided in schools. An alternative explanation for lower non-drug use in the UK could be difficulties seeking support or unclear referral pathways in the UK healthcare system, similar to those outlined above for drug treatments too. Additionally, Applied Behaviour Analysis (ABA) is an approach to behavioural therapy used far more regularly in the USA than Europe ${ }^{46}$. Suggested reasons for this include a lack of consensus on its effectiveness, differences in numbers of trained practitioners and cultural differences regarding the appropriateness of techniques used ${ }^{47}$.

The main hypothesis tests in Chapter 2.3 regarded associations between geography and insurance types on the uptake of non-drug treatments. We found no significant differences in levels of treatment use between private and Medicaid insurances, which is contrary to historical evidence of higher healthcare use in Medicaid ${ }^{48,49}$. Following the recent introduction of many state-level insurance mandates, requiring private insurance companies to cover ASD services by law, other studies have found significant increases in outpatient service expenditure for privately insured ASD patients ${ }^{48,50}$. Regarding geography, a significantly higher proportion of children in metropolitan areas versus nonmetropolitan areas received SLT (72\% vs 65\%) and behavioural therapy (57\% vs $46 \%$ ). The most widely reported barrier to care in rural areas was a lack of available local services.

The vast majority of caregivers commonly reported themselves as being the main coordinator of care, yet only 30\% accessed a caregiver education program. The median intensity of child-directed treatment was 6 hours per week, despite some authors recommending at least 25 hours per week to achieve optimal outcomes ${ }^{51}$. 


\section{Safety studies}

The aim of Chapter 3 was to explore possible unintended effects of commonly used drug treatments in ASD children.

In Chapter 3.1, we found no association between serious cardiovascular (SCV) events and the prescription of stimulants or atomoxetine: so-called "ADHD medications". From a cohort of 326,221 ASD children identified in USA claims data, we compared 48 patients who experienced a SCV event with 480 randomly selected age and sex matched control patients who had not experienced an event. Only $13 \%$ of the cases were currently exposed to ADHD medications versus $22 \%$ of controls, meaning that ADHD medication appeared to play no significant role in which patients would experience a SCV event (odds ratio and 95\% confidence interval (OR): $0.49(0.20-1.20)$ ).

There were other differences between the cases and controls however. Perhaps most striking was that $19 \%$ of the children with a SCV event had underlying congenital heart conditions compared to only $2 \%$ of the controls. This compares to around $1 \%$ in the wider general population ${ }^{52}$. Due to the way we extracted this information from the database, it is evident that the underlying conditions were known at least 30 days prior to the SCV event. An open question therefore is why some children with underlying heart conditions were exposed to ADHD medication, despite previous recommendations ${ }^{53-56}$ and warnings on package inserts ${ }^{57-60}$ to not use these medications in patients with structural cardiac abnormalities.

In a sensitivity analysis, we excluded cases and matched controls with such underlying risk factors, but still found no association between SCV events and ADHD medication exposure. With hindsight, a more meaningful analysis might have actually been to exclude patients without the underlying risk factors. This would have helped to elucidate if ADHD medication actually compounded the risk of SCV events in this vulnerable group of patients, or if the risk was mainly down the underlying heart conditions alone. Such a research question is an example of what would not be ethical to test in a randomised study, but where retrospective "real life" clinical practice data can be used instead. In a series of other sensitivity analyses, we controlled for underlying risk factors using other methodologies. One of the methods used was novel and is discussed later in the methodological considerations section of this chapter. In all analyses, our findings stayed the same. There were no associations found between SCV events and ADHD medications.

Supporting the above findings, we also found no association between SCV events and ADHD medication in a larger, parallel group of children with ADHD. The results were largely constant with previous observational studies from literature in the wider child and adolescent population ${ }^{55,61,62}$, but add considerable value given the recency of data and the large number of patients identified. ASD and ADHD patients combined, we studied 234 SCV events versus 81 events in the largest previous study with comparable outcomes ${ }^{62}$. 
The overall incidence rate of SCV events was extremely low but comparable to other studies ${ }^{61,62}$. Our estimates per 100,000 person-years were 5.6 in the ASD cohort and 3.1 in the ADHD cohort. These are consistent with the rates observed in other cohorts primarily comprised of ADHD children and adolescents in claims databases (2.8 to 3.1 per 100,000 61,62).

In Chapter 3.2, the aim was to compare the risk of bone fractures in children with ASD who were exposed to either risperidone or aripiprazole. Using claims data from the USA, we retrospectively identified 3,312 patients exposed to each medication, and found that over the whole duration of available follow-up there was a $40 \%$ lower risk of fracture in the risperidone group (hazard ratio and 95\% confidence interval (HR): 0.60 (0.440.83)). Risks were comparable between groups for the first 180 days on treatment, but significantly higher in the aripiprazole group thereafter. Extremity fractures drove most of the increased risk. Results were stable regardless of patient gender, but risk differences widened further for children aged 10 years or younger.

These results cast doubt on previously proposed mechanisms by which antipsychotics might affect bone health. Firstly, drug-induced hyperprolactinemia has been proposed as a possible mechanism leading to fractures ${ }^{63,64}$. Yet, of the drugs we studied, only risperidone increases prolactin levels while aripiprazole actually decreases prolactin levels ${ }^{65,66}$ and it was with aripiprazole that we observed increased fracture risk. Secondly, it does not appear that increased somnolence or drowsiness increased the risk either, as these adverse effects have also previously been reported more often under treatment with risperidone ${ }^{67,68}$ than aripiprazole ${ }^{69,70}$.

Clearly, more work is needed to understand other mechanisms and risk factors that may have contributed to our findings. A potential weakness of our study was that median follow-up was only around 10 months (interquartile range: 4 to 12 months) in both treatment groups. On the other hand, this duration must be reflective of real world clinical practice, and the reasons for discontinuation were comparable between groups (as far as can be ascertained from claims data).

\section{Autism Impact Measure}

The goal of Chapter 4 was to test the validity of the Autism Impact Measure (AIM) in children. We did this by inviting over 5,000 parents and caregivers of children with ASD to complete the AIM online, via the SPARK online research initiative ${ }^{71}$. We confirmed previous findings ${ }^{72}$ that the questionnaire has good psychometric properties, and covers five distinct symptom domains (Communication, Social Reciprocity, Repetitive Behavior, Atypical Behavior, and Peer Interaction) as well as the option to study a "total" score and/or the "frequency" or "impact" of symptoms only. We also showed the AIM has high internal and external validity, and can discriminate between "known-groups" of children with different symptom severity. This means that the AIM can be reliably used to study 
different aspects of ASD development over time, and/or to assess the effectiveness of different treatments too. We estimated clinically important responder (CIR) thresholds, which will help with future study design and clinical interpretation of the results.

Another novel aspect was that we demonstrated the AIM can be administered electronically, with similar validity on mobile and computer devices, taking an average of just over 7 minutes to complete. This is clearly an advantage of caregiver symptom report over other available tools that require trained personal and a much longer duration to administer. In ASD, direct patient reported outcomes would be more difficult to obtain. This is due to a range of verbal abilities and the general difficulties autistic children face, including communication and identifying and expressing feelings ${ }^{73}$. The age range in which we validated the AIM was 3-17 years.

\section{Methodological considerations}

As with any type of research, it is important to reflect critically on methodological limitations encountered in this thesis. By their nature, epidemiological studies may be prone to different kinds of bias, which can produce erroneous results or conclusions. Bias should be restricted wherever possible and at a minimum be properly acknowledged so that results can be taken in context. Bias in epidemiological studies can generally be grouped into three types: information bias, selection bias and confounding ${ }^{74,75}$. We will discuss specific issues related to these three types of bias below.

\section{Information bias}

Information bias occurs when data is incorrectly specified in a database. Here we discuss the accuracy of identifying eligible patients for study, as well as the definition of key exposure and outcome variables. The most profound issue would be if we suspected a differential pattern of information bias among different groups being compared.

\section{Identification of ASD}

Perhaps the most important aspect of any study is the correct identification of eligible patients. Widely used standard diagnostic criteria for autism are found in the Diagnostic and Statistical Manual of Mental Disorders (DSM), which is periodically updated by the American Psychiatric Association (APA). However, throughout secondary data use studies in this thesis, we needed to use different coding systems in order identify patients.

Since 2013 , the DSM-V ${ }^{76}$ has been the most recently published version, and is the first version to replace previously established subtypes of autism with the single term "autism spectrum disorder". The DSM-IV, first published in 1994 and revised in $2000^{77}$, included a broad category of "pervasive developmental disorder" (PPD). This included subtypes of autism including autistic disorder (the "classical" subtype); Asperger syndrome (so-called 
"high-functioning" autism) ${ }^{78,79}$; childhood disintegrative disorder (least common; where development regresses after typical early childhood ${ }^{80}$ ); and pervasive developmental disorder, not otherwise specified (PDD-NOS). The change from DSM-IV to DSM-V was meant to make autism diagnoses more consistent and better reflect that there are currently no known biological differences to separate these different subtypes ${ }^{81}$. There was expected to be no significant changes concerning the diagnosed prevalence of autism in real-life practice ${ }^{82}$.

Our data sources do not provide data on the comprehensiveness of the underlying diagnostic process, which may differ between healthcare systems or clinics. To arrive at a clinical diagnosis of ASD in clinical practice, this usually requires a thorough evaluation of core and associated symptoms by a developmental paediatrician, psychiatrist, neuropsychologist or other ASD specialist. Aims of the evaluation typically include establishing if symptoms align with published diagnostic criteria, whether psychiatric comorbidities are present, and the specific neurodevelopmental profile ${ }^{83}$. We do not have information on which diagnostic tools were used, such as the Autism Diagnostic Observation Schedule (ADOS) ${ }^{84}$, the Autism Diagnostic Interview-Revised (ADI-R) ${ }^{85}$ or the Childhood Autism Rating Scale (CARS) ${ }^{86}$.

In the USA, in insurance claims databases, physicians and facilities complete billing forms using a nationally modified version of the International Classification of Diseases coding system (ICD-CM). Version 9 (ICD-9-CM) was used until September 2015, when it was replaced by version 10 (ICD-10-CM). Both systems are similar to the DSM-IV, and present subtypes of PPD rather than the all-encompassing autism spectrum disorder. The main difference between ICD-9-CM and ICD-10-CM is that ICD-10-CM includes Asperger syndrome. As insurance forms are used for reimbursement purposes rather than a precise documentation of medical history, a single recorded ICD code does not always denote a clinical diagnosis. It could instead represent any reason for consultation on this topic including, for example, a patient complaint or diagnostic testing.

Fortunately, a previous validation study by Burke et al ${ }^{87}$ evaluated how often patients with ICD-9-CM codes for autism in insurance claims also had recorded evidence of autism in their medical charts. The study was set between 2001 and 2009. Investigators identified 432 patients from a commercial USA insurance database using ICD-9-CM codes (299.0x, 299.8x and 299.9x). An expert clinician reviewer with training on ASD chart abstraction for national prevalence estimates then confirmed if retrieved medical charts for the patient also contained evidence of ASD. There were two levels of certainty defined from the charts: either a description "highly indicative" of a DSM-IV diagnosis (level 1 criteria), or wider evidence of behavioural, social or communication deficits in the charts, consistent with the DSM-IV, but not enough to meet level 1 criteria (level 2). The study found that requiring two or more ICD-9-CM codes achieved a level 1 positive predictive value (PPV) of $61 \%$ and a level 2 PPV of $87 \%$. These were much higher than 
when requiring just one ICD-9-CM diagnosis code (43\% and 74\%). Hence, requiring two ICD codes formed the basis of the algorithm we used to identify ASD patients from claims databases throughout this thesis. The exception was Chapter 3.2 in which only one ICD code for ASD was required, because patients were also required to have a dispense of risperidone or aripiprazole, which itself is likely a confirmation of ASD.

While the Burke et al validation study was for ICD-9-CM, we assumed their applicability for ICD-10-CM too, which came into effect for billing purposes in September 2015. ICD10-CM codes used were similar to ICD-9-CM in that they corresponded to autism/autistic disorder (F84.0), and other/unspecified PPD (F84.8 and F84.9). The difference in ICD$10-C M$ is that it also included Asperger's syndrome (F84.5) which was not present in ICD-9-CM. Differences in ICD versions would not have affected Chapter 2.1, which was set in calendar year 2014 and prior to ICD-10-CM adoption. However, Chapter 3.1 was set between 2000 and 2016, and Chapter 3.2 was set between 2013 and 2018, which spanned the version change. Both studies were comparative in nature and calendar date was used as a matching variable in both. Therefore, any differences in diagnostic criteria over time were accounted for in comparative analyses. Naturally, further validation studies for ICD-10-CM should be welcomed in the future. ICD-11 will be the first version to refer to the autism spectrum but this is not expected for release until 2022 and its adoption in USA insurance data will likely be even later.

Of note, we also excluded patients with an ICD code for Rett's syndrome or who were under the age of 2 to 3 years in order to reflect diagnostic uncertainties ${ }^{2,4,87}$. Additionally, as per the validation study by Burke et al, we did not include ICD codes for childhood disintegrative disorder (CDD), which authors described as clinically distinct from the other ASD. This means that patients who would have previously met such criteria were not represented in our MarketScan studies. This likely has little impact on the external validity of our studies as a whole however, as CDD accounted for only around one in every 60 to 175 cases of autism, under the old DSM-IV definitions ${ }^{80}$.

Since the mid-1990s, the UK CPRD database has used the Read code system for documentation of clinical symptoms and diagnoses. As electronic medical records are used to document more precise medical history and symptoms than claims data, two previous validation studies showed that requiring just one Read code for autism in the CPRD database carries a high PPV (over 90\%) ${ }^{88,89}$. The first study by Fombonne et al ${ }^{88,90}$, which evaluated 318 Read code-identified children born between 1973 and 1997, found a 93\% PPV by making a detailed comparison between medical charts and the DSM-IV criteria. The most recent study, by Hagberg and Jick ${ }^{89}$ demonstrated that a reduced list of Read codes carried a 92\% PPV versus medical charts between 1990 and 2014. This is probably because the most common Read codes in their study (autism, autistic disorder and Asperger syndrome) also identified most patients in the study by Fombonne et al. However, Hagberg and Jick also included the term autism spectrum disorder, which Fromebone et al 
did not. Around 25\% of the patients in the Hagberg and Jick study were diagnosed after 2013, coinciding with when the concept of the autism spectrum was introduced in DSM-V. In their study, authors demonstrated that use of the Asperger syndrome code decreased from approximately 40\% of patients born in 1995 (the year after the DSM-IV was first published) to around $10 \%$ of patients born in 2005 , and to only $2 \%$ of patients born after 2010. In Chapter 2.2, we used the codes produced by Fombonne et al but also included the Read code for autism spectrum disorder, hence creating the most comprehensive list, while being reassured that the PPV remained high. We also excluded patients with Rett's syndrome codes in the CPRD study, but the code list included CDD, unlike the MarketScan studies. The Read code system, historically used for clinical terminology in the UK, is currently undergoing a transition to the SNOMED system ${ }^{91}$. Hence, further validation of the SNOMED codes will also be needed in the future.

High PPVs in both MarketScan claims data and the CPRD means we can be confident most patients identified in our studies are true cases. However, it is much harder to know the sensitivity of the code lists used. Perhaps some eligible patients with ASD were excluded incorrectly from our studies. Probably more patients in the USA were excluded than patients in the UK, due to the requirement of two codes in the USA databases. Potentially this is another reason why a higher proportion of patients in the USA received treatments, as milder cases with just one ASD code may not have been included. The extent of this misclassification is very difficult to assess given the data available.

Unfortunately, in both CPRD and MarketScan databases, it is not possible to ascertain information on the severity or specific symptoms of patients. None of the validation studies mentioned above benchmarked against established clinical diagnostic instruments 92. Finally, none of the validation studies included an adult population. This means that the accuracy by which adult patients were included in our studies is less clear. This affects Chapter 2.1 and Chapter 2.2 only. Only by the DSM-III, first published in 1980, was autism first recognised as a distinct disorder to schizophrenia ${ }^{93}$. That said, contemporary medical records, which were used in our studies (all studies used codes entered between 2000 and 2018), will probably reflect contemporary diagnostic criteria regardless of a patients initial diagnosis.

In the SPARK cohort (Chapter 2.3 and Chapter 4.1), the caregiver-report of ASD diagnosis is assumed highly valid as per other previous online studies (e.g. the Interactive Autism Network) ${ }^{71}$. Recruitment for many of the patients in SPARK was also in collaboration with clinical sites to increase chances that participants had a valid diagnosis. As fielding of our survey was in the DSM-V era, the majority of caregivers reported autism spectrum disorder as the main diagnostic label (74\%). The DSM-V grouping is supposed to emphasise similarities between ASD patients rather than differences, as well as make diagnosis easier 46. However, this does make it more difficult to study subgroups, and assess the external validity our findings compared to previous studies. 
Overall, for the studies in this thesis based on SPARK, we have a better characterisation of patients such as caregiver reported age at diagnosis, severity of symptoms and verbal ability. The possible limitation for the SPARK database is its representativeness of the wider ASD population, given that this is a survey-sample. The topic of survey representativeness is discussed in detail later in this chapter. For the drug utilisation studies, detailed patient characteristics or even the certainty of a true ASD diagnosis is not available in the MarketScan or CPRD data. Nonetheless, given the above, we have around an 87\% PPV in MarketScan data and a 92-93\% PPV in CPRD. Furthermore, as these cohorts are extracted from population-based data, they are likely more representative of the whole autism spectrum. Unfortunately, we cannot study subgroups in these data, and severity of symptoms could be an important missing confounder for the comparative studies. Confounding is also discussed later in the current chapter.

\section{Exposure data}

Other key variables to ascertain correctly are the exposures of interest. In MarketScan claims and CPRD data, exposure data simply reflect if prescriptions have been dispensed or issued, respectively. It is not possible to know if patients actually took the medication as prescribed.

Identification of "new users" of risperidone and aripiprazole in Chapter 3.2 was only possible via an algorithm, requiring at least one year of prior continual enrolment in the MarketScan database without any record of antipsychotic prescription. There was perhaps slightly more chance of incorrectly specifying patients in the aripiprazole group as new users, given the age at index in this unmatched group was 11.3 years opposed to 9.3 years for risperidone. On the other hand, the mean prior enrolment in the database without treatment was around 3 years in both treatment arms, so the chances of misspecification were small and differences between groups would be negligible.

A general concern regarding the CPRD data used in Chapter 2.2 could be that it that it only covers the primary care setting. Indeed NICE guidelines for ASD generally suggest referral to specialist care before prescription of psychotropic drugs, and especially for children ${ }^{10,11}$. Nevertheless, the level of underreporting is likely to be small. This is because specialists generally determine which medication is appropriate, but ask the GP to actually write the prescription ${ }^{94}$. Additionally, even when prescriptions are started outside of primary care, the nature of the GP "gatekeeper" system in the UK means that repeat prescriptions will normally be managed in primary care, and an account of what happened in secondary or tertiary care will be sent back to the GP for record keeping. The CPRD database will also cover patients permanently living in long-term residential facilities, so long as they are not classified as hospitals ${ }^{94}$. Since 2019, Public Health England have used another primary care database (The Health Improvement Network: THIN) which partly overlaps with CPRD, to monitor the success of its national program on the reduction of psychotropic drug use in people with ASD or a learning disability ${ }^{95}$. 


\section{Key outcomes}

In insurance claims data, multiple diagnosis codes can be recorded on each billing form. The MarketScan database contains up to five diagnosis codes from inpatient and outpatient physician forms (usually the CMS 1500 form) and up to nine diagnosis codes from facility forms (usually the UBO4 form). In the event of an inpatient stay, a primary diagnosis field is also populated, which usually corresponds with the principal discharge code ${ }^{96}$.

When identifying serious cardiovascular (SCV) events in Chapter 3.1, we initially searched for relevant ICD codes in all of the diagnosis fields. This naïve approach resulted in us estimating incidence rates around 30 times higher than rates previously reported 61,62 and prompted a more careful look at validation studies that linked claims data to confirmed SCV events in electronic medical records. Literature reviews show that by only considering cases with ICD codes in the primary diagnosis field from an inpatient visit, can achieve a PPV of at least $85 \%$ in the majority cases ${ }^{97-101}$. According to studies which reported it, this also keeps sensitivity relatively high ${ }^{99,100}$. Application of these more stringent criteria in our study resulted in SCV event rates completely aligned with those previously reported.

This highlights the importance of such validation studies for accurately identifying diagnoses in claims data. A limitation of the MarketScan claims data that cannot be overcome however, is that deaths outside of hospital are not recorded. As a small proportion of sudden SCV events may have led to death without a hospital visit, these events would likely have been missed in our study. Missing death information is unlikely to have had an impact on Chapter 3.2, as we censored patients in the Cox model when they left the database regardless of the reason (which is undocumented). Mortality rate in this group of young patients is expected to be very low.

Claims data have also been shown to have high PPV for identifying fractures ${ }^{102-105}$. However, literature is lacking to confirm this specifically in children, and differences in accuracy exist by fracture site ${ }^{104,105}$. For the study Chapter 3.2, we should be reassured in the accuracy of identifying true fractures, as over $90 \%$ of the diagnosis codes we identified were recorded in the first diagnosis field on billing forms. Additionally, the incidence rates were broadly similar to those studied the general child population and in other types of databases ${ }^{106}$. Importantly, for comparative purposes, there is no obvious reason why fractures would be recorded differently between patients exposed to risperidone or aripiprazole.

\section{Recall bias}

Recall bias refers to a situation in which a respondent incorrectly recalls what happened in the past. A strength of data from EMR or claims databases is that information is collected routinely, and usually during or shortly after consultation with the patient, so recall bias is not an issue. In contrast, recall bias can be problematic in surveys. 
During the SPARK survey conducted in Chapter 2.3, caregivers were asked to recall which treatments and services their child had received in the past year. Most of the non-drug treatments were reported as still ongoing (61-89\% depending on type of treatment) and perhaps there is more difficulty recalling services received further in the past. Due to the close involvement of caregivers in coordinating care however ${ }^{107}$, we expect them to recall this information well overall. There is no obvious reason why recall bias would be different based on geography or insurance status.

We also surveyed caregivers from the SPARK platform in Chapter 4.1. The AIM questionnaire only asks caregivers to recall their dependants' symptoms over the past two weeks, so recall bias seems less of a concern. A two week recall period also offers the option of more frequent and independent measurements to be collected over time.

\section{Selection bias}

Selection bias occurs when a sample selected for a study are not representative of the underlying population, or when there are systematic reasons that determine why patients are more or less likely selected into different comparison groups. In such scenarios, results may not be generalisable, or biased estimates may be produced when comparing groups.

\section{Survey representativeness}

Perhaps the most obvious and intuitive example of selection bias, is when respondents of a survey are not representative of the wider population which were intended to be studied. This concept is applicable to studies in this thesis that surveyed participants from the SPARK online ASD platform (Chapter 2.3 and Chapter 4.1).

Regarding parental education levels, we had around twice as many respondents that completed a graduate degree than in the general USA adult population (64\% vs 33\% ${ }^{108}$ ). The most commonly reported family income in our study was between $\$ 50,000$ and $\$ 75,000$ in 2017, which coincided with the median USA household income of $\$ 61,423$ in the same year ${ }^{109}$. However this is still likely slightly higher than a typical family with an autistic child due to work productivity loss ${ }^{110,111}$. Those with higher income and education are perhaps more likely to be aware of and secure access to treatments for their children, via either advocacy or out-of-pocket payments ${ }^{9,112}$.

An additional inclusion criterion for our survey was that participants needed to understand English. This presumably excluded 9\% of people in the USA who speak English "less than well" according to the 2009-2013 US Annual Community Survey ${ }^{113}$. More of the children in our survey were of White/non-Hispanic race than in the general ASD population (69\% vs 53\%) but were similar in terms of age at diagnosis, sex and caregiver-reported use of medications for ASD ${ }^{114}$. 
The online format of the survey would have removed any potential barrier to participation in terms of travel time to research sites. Additionally, in the USA, only $3 \%$ of adults aged 30-49 years (the majority age range of caregiver respondents) do not use the internet ${ }^{115}$ so this should have widened the opportunity to take part. Any small residual differences in internet availability between rural and metropolitan areas would likely have negligible impact on our findings. The study actually had participants from all 50 states, plus overseas territories. Despite some clear limitations, the size and diversity of our sample is unprecedented for this kind of study, as is the SPARK cohort in general ${ }^{71}$. With regard to selection by parental education, the results in our study can still be compared to work carried out by Salomone et al ${ }^{9}$ in Europe which also had high rates of graduate level education among caregiver respondents (63\% vs 64\%).

\section{Biases in case-control studies}

In a case-control study, we have two opportunities to introduce selection bias: first, when picking the cases, and then when selecting the controls. Here we explore these concepts in the context of Chapter 3.1.

First, we selected cases who experienced a serious cardiovascular event (SCV). The intent was to see if these patients were more commonly prescribed ADHD medication than controls in the time leading up to the event. Another characteristic common to many of these patients however was the presence of other underlying cardiovascular comorbidities (19\% had underlying a congenital heart condition). As the ADHD medications of interest carry class wide SCV warnings ${ }^{57-60}$, the expectation is that patients with underlying cardiovascular risk are actually less likely to receive treatment. This "reverse causality" could have paradoxically given rise to what looks like a protective effect of ADHD medications on SCV events (the point estimate for odds ratio was 0.49). In fact, this type of bias does not only influence selection of cases in case-control studies ${ }^{116}$, but is called confounding by (contra-) indication and impacts cohort studies too ${ }^{117}$.

The controls were sampled according to the incidence density sampling technique ${ }^{118}$. This means that at the time of the cases' event, all patients in the cohort yet to experience the event ("at-risk") had an equal chance of being selected as a control. This is important step to not introduce bias. While the probability is small, this method means is possible for some cases to also be a considered a control for another case, using data prior to their own outcome of interest.

To make cases and controls more comparable, we matched on age, sex and insurance type. This accounts for the some of the confounding factors, however, we still needed to adjust for other factors at the analysis stage. A common issue when deriving covariates in casecontrol studies is the inclusion of information that happened post exposure, potentially on the causal pathway between exposure and outcome and therefore introducing bias 119,120 . We tried to account for this in our covariate derivations, by ignoring data during 
a 30-day lead-in period prior to the event. For example, this means that if a patient had a cardiology visit in the last 30 days prior to event, then we ignored it when deriving the cardiology visit covariate. This is because there is a good chance the cardiology visit happened after the exposure, and may be causally related to exposure itself. Justification for 30 days was based on the belief that the most likely exposure effect would be acute ${ }^{121}$. It is an assumption however, and must be acknowledged.

For these reasons and others, there are strong critics of the case-control design, and selfcontrolled designs have been suggested as an alternative approach ${ }^{120}$. Self-controlled designs have an inbuilt advantage of controlling for within subject characteristics during periods of exposure and non-exposure. Disadvantages however include that results are only representative of patients who experience an event. Exposure time after an event is also counted ${ }^{122}$, and this is likely to introduce a different kind of bias because experiencing serious or chronic adverse effects will likely make future use of the same treatment less likely ${ }^{123}$. In an ironic example, a study that tried to expose the flaws of case-control designs, included exposure time after the event of ulcerative colitis onset (a chronic disease) ${ }^{120}$. Breaking up post-event time into periods of high and low exposure windows has been suggested, but the approach still only makes sense with acute, nonsevere outcomes and transient exposures ${ }^{122}$. Cohort studies are another option but they require repeated assessments of exposure status, and potentially covariates, over time. This makes cohort studies less operationally convenient for testing assumptions about the proximity of exposure to the outcome of interest ${ }^{119}$. For example we also performed analyses based on exposure in the previous 90 days prior to the SCV event and an "ever exposed" analysis, without needing to redefine which patient to include in the analyses or re-derive any other variables.

A final type of bias to consider in the case-control design is immeasurable time bias. This time-related bias occurs in scenarios where it would be impossible to observe exposures during the pre-event exposure window ${ }^{124}$. For example, if the event of interest led to cases being in hospital during the days prior to the event, then outpatient prescriptions to the patient would not be possible. This type of bias would be related to the contraindication bias mentioned above for Chapter 3.1, as cases with poorest underlying health would be more likely hospitalised prior to the event. In our study design however, such hospitalisations would not be directly related to SCV events as we used the first SCV inpatient hospitalisation record to denote the event date. Additionally, the proposed mechanism for ADHD medications leading to SCV events means that admission to hospital would be very shortly after an exposure.

Ultimately, there are some clear limitations in Chapter 3.1, but measures were taken to limit them as much as possible, and sensitivity analyses testing different assumptions all provided consistent results. In a future analysis, it would be interesting to empirically check for evidence of reverse causality by assessing negative control exposures on the same 
selected cases and controls ${ }^{120,125}$. Additionally, a future study aiming to replicate findings using a cohort study approach would be a good experiment too. Both study designs should produce the same estimates in theory ${ }^{119}$.

\section{Biases in cohort studies}

The objective of Chapter 3.2 was to compare the safety of two drugs head-to-head over time. The head-to-head approach of two active treatments made it possible to emulate certain aspects of a controlled "target trial" ${ }^{126}$. This is because an index date (start of follow-up time) could be easily defined in both groups as the time of new treatment initiation. This is called a new-user design ${ }^{127}$. A clearly defined index date not only provides a clear start of follow-up, but also facilitates easy application of eligibility criteria at this specific point in time. This is similar in principal to clinical trials, where all patients who meet explicit eligibility criteria at baseline are included as part of the intention-to-treat (ITT) analysis, regardless of their later compliance with the treatment regimen ${ }^{128}$.

Other time-related effects, still possibly related to treatment, such as differential loss to follow-up or discontinuation of treatment can also be easily observed in this design. In our study, we noticed that patients in both the aripiprazole and risperidone groups were followed for similar amounts of time, and had similar reasons for leaving the cohort. Hence, there was no obvious differential loss to follow-up, other than in the differential rate of fractures. In other studies where reasons for loss to follow-up are related to the outcomes of interest, this can cause spurious results ${ }^{129}$. In such cases, competing risk time to event models can be applied ${ }^{130}$. Examples of competing risks include receiving a kidney transplant when studying time to death for patients with kidney disease ${ }^{131}$ or finding a new stable partner when studying HIV infection rates among sex workers ${ }^{132}$. In our study, a potential competing risk for bone fracture could be death due to accidents, which is not possible to evaluate in our database, as death is not well recorded. It likely has little impact on our results however, given that mortality in this young cohort is expected to be extremely rare. Another potential competing risk would be switching to the opposite treatment of interest (i.e. from aripiprazole to risperidone or vice versa). However, we know that the proportion of patients switching was similar in both treatment arms so this unlikely resulted in a biased comparison.

In other study designs, where assignment of an index date for patients is less clear, time related biases are usually harder to identify. It is especially difficult to assign an appropriate index date to patients who receive no treatment; hence, a comparison of treatment versus no exposure needs careful attention. In selecting the follow-up period for such studies, immortal time - defined as a studied period in which the outcome of interest could not have occurred - should be avoided ${ }^{133}$. A classic example of this is a study that counted follow-up time from a given calendar date rather than beginning of a treatment regimen ${ }^{134}$. The study compared the number of drug prescriptions received over time with an outcome of future hospitalisation. However, for patients who went on to receive 
treatment, the time between start of follow-up and first prescription is immortal as no hospitalisation could occur in this time. This artificially lowered the rate of hospitalisations in the exposed group ${ }^{133}$.

Another way in which immortal time may be introduced is based on exposure hierarchy ${ }^{133}$. In such circumstances, patients ever exposed during the observation period are compared to patients never exposed during the whole observation period. If patients are then excluded from the ever-exposed group based on a prior event, then these events are not properly counted towards the non-exposed time. This phenomenon is clearest when the event of interest is death. For example, in a CPRD study, the rate of death was compared between patients exposed to a combination of inhaled corticosteroids and long-acting beta-agonists versus patients exposed to a bronchodilator ${ }^{135}$. Some patients included in the combination therapy group had previously been exposed to a bronchodilator. If patients had died in the bronchodilator exposure period, they could not ever have been included in the combination therapy group ${ }^{133}$.

To reiterate, these immortal time issues will not have influenced Chapter 3.2 in its current state due to the new-user design and comparison of two cohorts with clearly defined index dates. In the future however, if extensions are made to study the effects of cumulative dose, or washout periods in which risk remains altered after treatment, then these exposures need to be accounted for carefully. For example, patients need to survive a period of current exposure in order to reach a period of previous exposure. Similarly, high cumulative doses will only be experienced after event free periods at lower doses. Time dependant Cox models have been suggested ${ }^{133}$ and demonstrated ${ }^{136}$ to correctly adjust for these time varying exposures. In the more complicated case that important covariates also vary over time and are dependent on prior exposure, then g-estimation or marginal structural models (MSM) are recommended ${ }^{137}$, as they take such conditional probabilities into account ${ }^{138,139}$. This would likely be the case in a study of fractures in ASD given the frequent use of other psychotropic drugs in this population.

In our experience, such models can quickly become extremely complex, both to define and interpret. If the main aim is to simply study effects of antipsychotic exposure versus no pharmacological drug exposure, then an alternative approach could be to define a reference group as new users of behavioural therapy. This solves the problem of defining an index date for a non-pharmacological exposure group, and arguably provides a more similar comparator group too, as patients are starting some form of treatment over no treatment at all. Naturally, other reasons for initiating non-drug therapy first over antipsychotic therapy would need to be considered. This then becomes a problem of bias by confounding rather than bias by selection. 


\section{Confounding}

Confounding refers to a process in which exposures and outcomes of interest are both influenced by a third "confounder" variable. Confounding is a serious problem in epidemiological research as it may result in finding spurious (not causal) estimates of association between exposures and outcomes if not properly addressed ${ }^{140}$. Without adjustment for confounders, effect estimates may be distorted in size or even reversed

${ }^{141}$. As discussed previously, some types of confounding can be introduced unintentionally at the study design phase. Commonly however, other confounding factors are impossible to eradicate at the design phase, as they are true reflections of real life situations and clinical practice. Identification of confounding variables typically requires disease area knowledge. Below we discuss specific methods used to adjust for confounding in the analysis stage of studies.

\section{Directed acyclic graphs}

Directed acyclic graphs (DAGs) are pictorial representations of assumed processes that gave rise to observed data. In epidemiology, they can be used to decide which minimum set of variables need to be adjusted for in analyses order to eliminate confounding and estimate unbiased associations between exposures and outcomes ${ }^{142}$. Contrary to common practice, it is not always desirable to simply adjust for all available variables, and doing so can actually introduce more bias in some circumstances ${ }^{143,144}$.

In Chapter 2.3, we used a DAG to consider the relationships among all variables assumed to influence the association between type of insurance coverage (private or Medicaid) and receiving treatment, or between geography (level of urbanisation) and receiving treatment. We graphically displayed the DAG and identified the minimal adjustment sets using free software ${ }^{145}$. We also confirmed the minimal adjustment sets using graphical criteria of the backdoor test for sufficiency ${ }^{142,146}$. While the full DAG is available in Chapter 2.3 (supplement S.2.3.1), key assumptions we made about important variables are displayed in Figure 5.1 and discussed below.

The first key variable in Figure 5.1 is household income. Household income clearly confounds the relationship between insurance type and treatment: that is, it clearly influences them both. Firstly, low earners qualify for Medicaid, while high earners will not. Secondly, a greater proportion of high earners would be able to pay for access to treatment directly ("out-of-pocket"). While income is likely influenced by geography - perhaps concerning the type of jobs available in the local area - it is less clear that a causal relationship exists in the other direction. Hence, income does not confound the relationship between geography and treatment.

Similar to household income, high levels of education strongly aids parents to seek out and advocate for their child's access to ASD services ${ }^{9}$, but as we assumed no relationship between parent education and geography, then parent education does not confound the 
relationship between geography and treatment either. We assumed there is likely an effect of parent education on insurance, however this is "through" the effect on income (also a proxy for job), and is unlikely to influence insurance type directly.

\section{Figure 5.1: Simplified causal diagram for key variables influencing non-drug treatment use}

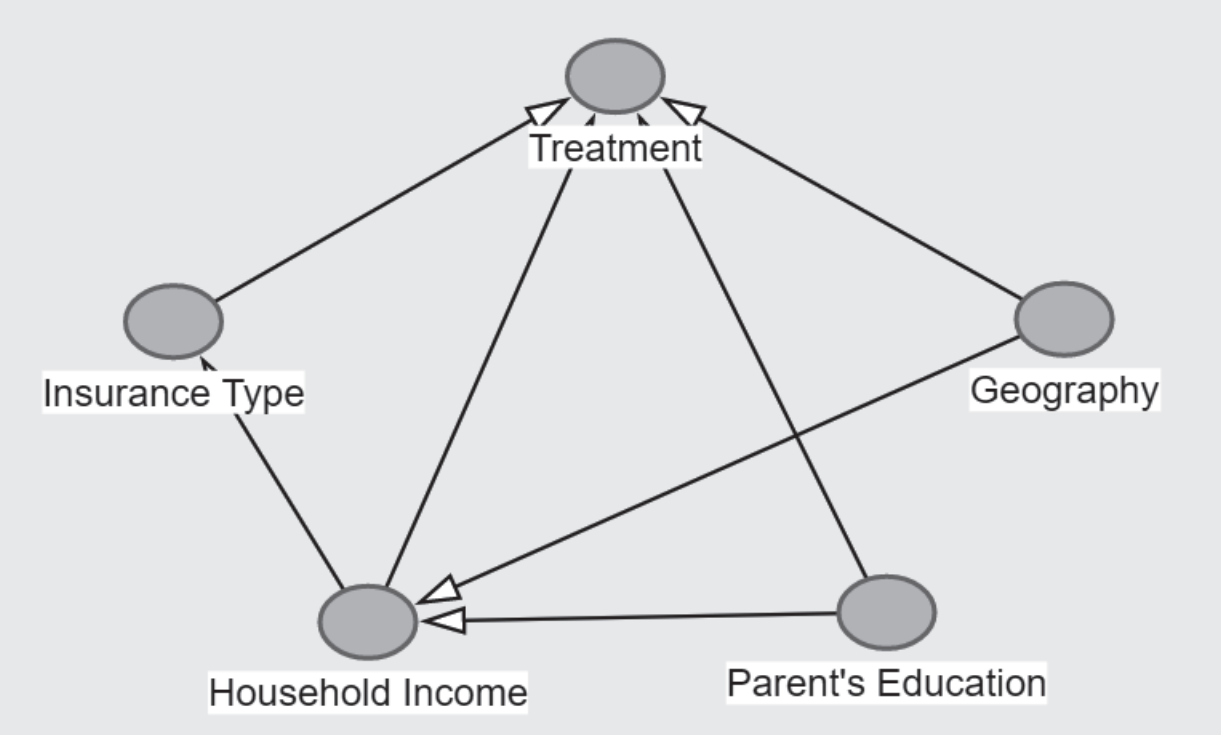

Insurance type = private or Medicaid; Geography = rural or urban location .

Full diagram available in Chapter 2.3 (supplement S.2.3.1).

The assumptions underlying this DAG led to the choice of variables that we simultaneously controlled for in Chapter 2.3. Naturally, these assumptions might be challenged, but we believe that this clear and open approach is part of good scientific practice, allowing replicability of findings and proper scrutiny in peer review. Other advantages to defining an a priori set of variables for statistical adjustment is that it can also aid study design (e.g. selecting the right dataset), increase statistical efficiency (only need to adjust for minimum set) ${ }^{147}$ and ensure control of type I error rates (through reduction of multiple testing with various adjustment sets).

Sometimes confounding can be so strong in a dataset, that statistical adjustment is not appropriate. For example, in our dataset, at the lower and upper strata of household income, children were almost exclusively enrolled into Medicaid $(<\$ 20,000)$ or in private insurance provided by employer (>\$9,000). We therefore restricted our analysis only to those families with an annual income between $\$ 20,000$ and $\$ 99,999$. Clearly, this means that results can only be interpreted in this more limited, middle-income group, but the approach was most appropriate in this context. 


\section{Propensity scores}

The fundamental reason that randomised control trials (RCTs) remain the gold standard method for evaluating treatment effects, is randomisation. Randomisation means that each person enrolled in a study has an equal chance of being assigned to each exposure group, independent of any other factors. By design therefore, all factors - including those that could influence outcomes - are balanced equally between treatment groups and confounding is not possible ${ }^{148,149}$.

In observational studies, various factors could play a role in reasons why people receive different treatments. Hence, when these factors are confounders, statistical adjustment is usually needed in order to remove their effect and estimate the "true" treatment effect. One commonly used method is the propensity score (PS). The basic idea of the PS method is to evaluate the probability of assignment to a given treatment as a function of observed patient characteristics ${ }^{150}$, and incorporate these estimates into subsequent analysis. Different methods for estimating the PS have been suggested. Most commonly, logistic regression is used, but other methods involving decision trees and other machine learning methods are also used ${ }^{151,152}$.

Once PSs have been estimated for different patients, they are typically (not exclusively) incorporated into analyses using one of two methods: matching or weighting. Matching involves comparing patients who have similar propensity scores (i.e. had similar probability of receiving the same treatment), but that actually received different treatments. This in essence achieves a "pseudo-randomised" subset of the entire available dataset, filtered on the most comparable patients. It is the method we used in Chapter 3.2 to compare risperidone and aripiprazole for fracture risk. Weighting on the other hand, can use data from all patients, but uses the propensity score as a way to assign more or less contribution of their information to the analysis. These weights are sometimes referred to as inverse probability weights (IPW). Weights can be assigned such that resultant weighted groups are more representative of a specific exposure group (thus estimating the average treatment effect in the exposed population: ATT); or, such that both groups are more representative of the overall source population (estimating the average treatment effect: ATE) ${ }^{153}$. In Chapter 2.3, we used the ATE weighting approach to compare if access to nondrug ASD treatments were more associated with private or Medicaid insurances. This seemed most appropriate, as policy makers are initially most likely interested to know if, on average, access to treatment is currently comparable across both types of insurance. This is opposed to the ATT, which would have estimated the possible effect of expanding Medicaid eligibility to all patients who are currently privately insured (or expanding private insurance, depending how we set the model up).

\section{Propensity score weighting in case-control studies}

Propensity score approaches are less commonly used in case-control studies. The main reason is that controls are typically sampled in some pre-defined ratio to the number of 
cases and hence the probability of being a case or control is fixed by design. As probabilities are fixed, the notion of estimating the true probability of being a case or a control only using information from the sample of cases and controls is flawed.

With that said, we employed a novel method in the case-control study in Chapter 3.1, with some theoretical motivation from propensity score inverse probability weighting. To justify the choice of this method, first we must bear in mind the end goal of propensity score techniques is to achieve covariate balance between comparison groups ${ }^{148}$. The propensity score is simply a means to arrive at such a junction. As such, an ideal method would be able to estimate weights that balance groups directly, without first estimating propensity scores. Fortunately, gradient boosted models (a type of machine learning method) are able to do just that ${ }^{154}$. Another advantage of using this approach is that many covariates can be controlled for without having to consider the functional form of their relationships to each other and to group assignment ${ }^{154}$. To our knowledge, this application of the boosted derived weighting scores is new in case-control studies.

Naturally, like with any new approach, it will be important to provide more reassurance on its validity before encouraging widespread use in other studies. A statistical proof that provides a full set of all assumptions would arguably be the best way to do this, at least from a theoretical point of view. In reality however, strict assumptions in such proofs rarely hold true when working with observational data, so empirical evidence that the method works will also be highly beneficial. Some of this was already achieved in Chapter 3.1. For example, the boosted-weights method produced results wholly consistent with the conditional logistic regression models, which is a more established method for covariate adjustment. Our results were also consistent with the majority of other studies that previously addressed a similar research question, including those which did not use a case-control study design. Moving forwards, more replication of results from other historical studies should also be undertaken. These should include a variety of disease areas and previous study designs. Additionally, evidence that that method correctly identifies relationships in simulated data would also provide a very credible argument. A combination of the above approaches should be carried out in order to generate a consensus that the new method is robust.

As the rationale for case-control studies in large administrate databases is declining ${ }^{120}$, acceptance of this method would most likely have biggest incremental impact outside of this setting.

\section{General comments on confounding}

In all comparative studies in this thesis, we propensity score adjusted for a large number of potential confounders and/or carefully selected a confounder set based on DAG theory. Fundamentally however, propensity scores (or any other adjustment method) cannot account for confounders unmeasured in the dataset ${ }^{148}$. Likewise, DAGs may reassure 
us that adjustment sets are correctly specified given assumptions, but they do require us to make assumptions in the first place. Hence, even with careful design and analysis, we should always be aware of potential residual confounding in all observational studies.

Recently, the concept of e-values have been introduced to the epidemiological literature ${ }^{155}$. An e-value denotes the minimum strength of a cofounder variable that would be needed to alter the observed association between exposures and outcomes ${ }^{155,156}$. Using free online software ${ }^{156,157}$, we calculated the minimum strength of an unmeasured confounder needed to alter the conclusions of Chapter 3.2 (i.e. to bring the upper bound of the confidence interval for the hazards ratio for fractures above 1). In this circumstance, the unmeasured confounder would need to alter both the choice of antipsychotic (aripiprazole or risperidone) and the risk of bone fractures by 1.7 times or more in order to change our conclusion. Hence, we can be confident even a small amount of residual confounding would not have changed our study conclusions. A general limitation of e-values is its underlying assumption that only unmeasured confounding may explain distorted findings in an observational study, thereby ignoring types of information or selection bias.

Instrumental variables have been proposed as a way to mimic "natural randomisation" via exploitation of natural phenomenon ${ }^{158,159}$ and negative controls are a technique that can help to identify unmeasured confounding in observational data ${ }^{125}$. Where feasible, both techniques could be used in the future to verify or dispute some of the inferences made in this thesis. These issues of confounding are not relevant in sufficiently powered RCTs due to the "magic of randomisation" 149.

\section{Overall implications and recommendations}

The results of Chapter 2 provided a comprehensive insight into real-world treatment utilisation patterns in ASD. We extended previous literature in this area by studying the adult ASD population as well as children, estimating treatment use outside the USA, assessing non-drug treatment use, and updating prior information by around 10 years compared to the other most recent studies. Our cohorts were mainly population-based, broadly representative, covered private and Medicaid insurances in the USA and were larger than any other previous studies. Overall, the levels of treatment utilisation were substantial: both for drug and non-drug services. Reflective of disease heterogeneity, this included a wide variety of treatments for core and non-core ASD symptoms, as well as associated psychiatric comorbidities, which were highly prevalent. This knowledge on treatment utilisation can be useful for ASD researchers planning or interpreting results of new studies, and for healthcare systems planning training and resourcing for ASD services in the coming years. 
Additionally, we studied factors associated with treatment use, in order to understand if certain groups of patients had more challenges in accessing appropriate treatments than others. Patients in the USA and UK were more likely to receive treatment in the presence of other psychiatric comorbidities, but this did not explain all of their use. In the USA for example, children in foster care were more likely to receive treatments. A recent policy experiment in Texas showed that multi-team screening interventions significantly reduced inappropriate (off-label) medication use in foster care children ${ }^{160}$ and hence similar programs should be considered elsewhere. Also in the USA, White patients were more likely to receive drug treatment than Black or Hispanic patients were. Historical racial or ethnic disparities in early ASD diagnosis rates have been narrowing in recent years ${ }^{161}$ but more should be done to continue this trend. We did not assess race differences in the UK studies. Additionally, further research should be done to understand other socialcultural reasons for racial differences in uptake and discontinuation rates of treatments, and address them where necessary. Recent widening of ASD private insurance mandates in the USA seems to have had little effect on addressing racial differences in treatment access ${ }^{162}$. However, expansion of the Medicaid Home and Community Based Services (HCBS) waiver - allowing patients to receive services in their own home or community - has shown greater potential ${ }^{163}$.

Specifically concerning non-drug treatments in the USA, we found no significant association between insurance type and the uptake of services. Our results therefore may indicate that private insurance ASD mandates have had an effect on levelling-up historical differences in access to ASD services between private and Medicaid insured patients. A study that specifically addressed this issue found expenditure on ASD outpatient services almost doubled among large, employer-sponsored plans following the introduction of the insurance mandate in Pennsylvania ${ }^{164}$. A specific limitation of our study is that we assessed associations on a USA-wide level, despite that Medicaid eligibility criteria and the scope of private insurance mandates vary by state ${ }^{165}$. Point estimates also mainly favoured Medicaid despite not being statistically significant. Further analysis on the state level should therefore be explored. This could partly be achieved in our own dataset, given that we had representation from all 50 states, plus overseas territories. However, we did not collect information prior to the introduction of mandates. Insurance claims data would offer an alternative approach, but with less detail on specific treatments received.

Another major finding, was that autistic children in non-metropolitan (rural) areas received fewer non-drug treatments and for fewer hours per week than peers in metropolitan areas. These included treatments that are more commonly provided inside and outside of school. Corresponding with this finding, more caregivers in rural communities reported a lack of locally available services as the biggest barrier to care. Telehealth has been suggested as one possible avenue to overcome the barriers of accessing specialist support in rural areas ${ }^{166}$. Indeed, online cognitive behaviour therapy has been shown effective in non-autistic children with anxiety ${ }^{167}$ and sleep disorders ${ }^{168}$, but these online 
services clearly would not be appropriate for all children with ASD. Naturally, parents and caregivers already commonly provide around the clock support for children with ASD, yet specific training to equip caregivers as interventionists has also been encouraged ${ }^{107}$. Training courses delivered online for parents may be more feasible, and initial research in this area has been promising ${ }^{169,170}$. Further research should also focus on the impact of such interventions on parent resources and stress levels, its possible impact on siblings 107,171 and how training can be tailored to meet various cultural views on parenting style 172. Other general limitations of our survey of non-drug treatment was that it attracted responses from an above-averagely educated parent group, and that treatment utilisation in adults with ASD were not studied. These limitations should also be addressed in future studies.

Although studies in Chapter 2 were not designed for direct comparison to one another, an unmissable difference was that pharmacological treatment use is around twice as high in the USA than in the UK. Perhaps contributing to this was possible underreporting of prescriptions made directly from secondary or tertiary care in Chapter 2.2. While the impact on our findings are likely minimal, they cannot be fully quantified without supplemental information from these settings. A linkage study between the CPRD and the Mental Health Dataset (MHDS) could provide this opportunity ${ }^{173}$. Additionally, patients were identified for studies using different coding systems and algorithms as discussed in the methodological considerations section of this chapter. Further validation of both the MarketScan and CPRD data for positive identification of ASD according to the most recent diagnostic criteria (DSM-V) and in adults is needed. Opportunities may lie in the linkage of these data to prospective cohort studies, for example the linkage of CPRD data to the Adult Autism Spectrum Cohort ${ }^{174}$.

Other potential reasons for differences in country prescription rates were laid out in the summary of findings in context section of this chapter. Briefly, differences in regulatory drug approvals likely explain some variation, but other factors such as differences in clinical guidelines, attitudes towards receiving drug treatment and differences in healthcare systems deserve further exploration. Healthcare insurance coverage in the USA has been discussed above. Specific concerns relating to the GP "gatekeeper" system in the UK are starting to be properly acknowledged and addressed too. For example, a 2016 government-commissioned report included recommendations to build a primary care autism register and to offer annual GP health checks for all autistic people ${ }^{175}$. A similar program is already in place that covers people aged 14 years or over with a learning disability ${ }^{176}$. Pilot programs will extend this over the coming years to additionally cover all people with autism as part of the NHS Long Term Plan ${ }^{177}$. The rationale is that these consultations will prompt proactive healthcare planning for ASD services, as well an opportunity to discuss other healthcare needs ${ }^{175}$. GPs should also be trained and reminded to make "reasonable adjustments" to accommodate the needs of autistic patients such as communicating in simplified language or scheduling appointments at the end of 
the day to avoid unnecessary sensory overload in busy waiting rooms ${ }^{176}$. On a similar note in May 2020, the Royal College of GPs (RCGP) and Autism Alliance UK published joint guidance on how to make the environment at GP practices more autism-friendly ${ }^{178}$. This is part of the RCGPS wider work on providing additional ASD training for GP staff, and tips for GPs and autistic patients on how to benefit most from consultations ${ }^{178,179}$. Such initiatives should be welcomed in order to make sure that all ASD patients and caregivers are comfortable seeking support when needed, and are reassured in their doctors ability to help them access appropriate available care.

While Chapter 2 of this thesis offered broad insights into system-wide treatment patterns, future choices about if to initiate specific treatments will always be made on an individual level. These choices should be based on all available information about an interventions potential risks and benefits. To contribute some available information regarding risks, Chapter 3 provided evaluations on potential side effects of commonly used treatments in ASD. We complimented this in Chapter 4 by assessing the suitability of a new outcome measure for quantifying potential benefits of different interventions.

Results of Chapter 3.1 demonstrated no association between SCV events and prior treatment with ADHD medications. Additionally, the incidence rate of such events was extremely low. These results should reassure physicians, patients and parents that for the vast majority of autistic children, these outcomes are highly improbable and not systematically linked to ADHD treatment. Children with serious underlying cardiac abnormalities were however at increased risk. Further observational studies should consider focusing on this subgroup, and evaluate if risk is independent of - or compounded by - ADHD medication in this specific group of vulnerable patients. Until the evidence is clear, physicians should continue to note class-wide warnings and make careful cardiac evaluations in such patients before a decision to prescribe these medications ${ }^{57-60}$.

Results of Chapter 3.2 demonstrated that over long periods of exposure in childhood, risperidone was associated with a $40 \%$ reduction in bone fractures compared to aripiprazole. Differences between treatments in children aged 2-10 years were most pronounced. These results are contrary to a theory ${ }^{63}$ linking antipsychotic-induced hyperprolactinemia with reduced bone mineral density and fractures, mainly in elderly populations ${ }^{180,181}$. Further validation studies in ASD children should therefore be carried out, and they should adjust for other potential confounding factors that were unavailable in our study. These include diet, BMI, physical activity and ASD severity ${ }^{182,183}$. Due to mechanistic uncertainties, future studies should ideally ascertain causes of the fracture too. Finally, future studies should assess dose effects, including the initial dose and changes over time (current and cumulative dose). Until more is understood about these mechanisms and patients most at risk, patients and physicians should continue to use aripiprazole as usual but be aware of this signal. 
The final major contribution of this thesis was the validation assessment of the Autism Impact Measure (AIM). Given the variety of available interventions already being used, plus those in development, having reliable and accurate ways to compare treatment benefits is of critical importance for decision making. Fortunately, we found that the AIM has good psychometric properties, and allows the report of core ASD symptoms including communication deficits, difficulties with social interactions and repetitive behaviours. It could therefore be a candidate endpoint for future clinical trials. As the AIM has other distinctive benefits of being caregiver-relevant, quick and inexpensive to administer remotely, then it should also be considered as suitable tool for real world monitoring of ASD symptoms too. Through such a mechanism, the evaluation of different therapeutic approaches beyond the duration and setting of clinical trials can inform treatment guidelines on a group level. It could also allow rapid feedback direct for effectively finding optimal treatment strategies on the individual level ${ }^{184}$. While we validated the AIM in children aged 3-17 years, further adaptation would likely be needed in order to facilitate its use in adults, given the phrasing of certain questions like "playing" with peers or "toys". Additionally, there may be benefit in assessing if the AIM is more or less valid in any specific subgroups, such as those stratified by age, verbal ability or intellectual disability.

While our study was the first to estimate thresholds of clinically important changes of the AIM, a weakness was that these estimates were only based on cross-sectional data. As we collected information via the SPARK online platform however, this offered a unique opportunity to re-survey the same caregivers for longitudinal data. Invitations for a second survey of these caregivers were sent in autumn 2018, approximately one year after the first survey. New findings related to clinically meaningful thresholds of AIM, and associations between changes in AIM scores, baseline treatment use, and caregiver reported burden are expected to be published soon ${ }^{185}$. The fact the SPARK platform as a whole has recruited 60,000 individuals with ASD and their families since forming in 2016 186,187 is testament to the incredible engagement and willingness of the ASD community to contribute to further research. All data from our studies in the SPARK cohort will be made available for linkage via the SPARK platform. The opportunity for future studies in the platform, either prospective or retrospective in nature, should not be missed.

\section{Final conclusions}

This thesis demonstrates that the treatment landscape in ASD is varied with many drug and non-drug treatment options commonly used. The likelihood of accessing and receiving these treatments is dependent on more than just health status, and is also associated with increased age, country of residence, female gender (in the UK), and urbanisation of residence, fee-for-service healthcare plans, race, and foster care status (in the USA). We showed that serious cardiovascular events were not associated with ADHD medication for most children with ASD. Compared to risperidone, long-term aripiprazole use in ASD 
was associated with increased risk of fractures, especially for children under age 10 years. Finally, the Autism Impact Measure (AIM) offers a valid, quick and inexpensive method for caregivers to report their child's severity of core autism symptoms.

In the coming years, as the treatment landscape in ASD continues to evolve, epidemiology and real world databases (both primary and secondary data use) will continue to compliment clinical trials in helping to understand real life experiences of how people with ASD access and use treatments, as well as the risks and benefits of doing so. 


\section{References}

1. Mandell, D. S. et al. Psychotropic medication use among Medicaid-enrolled children with autism spectrum disorders. Pediatrics 121, e441-448 (2008).

2. Oswald, D. P. \& Sonenklar, N. A. Medication use among children with autism spectrum disorders. J. Child Adolesc. Psychopharmacol. 17, 348-355 (2007).

3. Schubart, J. R., Camacho, F. \& Leslie, D. Psychotropic medication trends among children and adolescents with autism spectrum disorder in the Medicaid program. Autism Int. J. Res. Pract. 18, 631-637 (2014).

4. Spencer, D.etal. Psychotropic medication use and polypharmacy in children with autism spectrum disorders. Pediatrics 132, 833-840 (2013).

5. Vohra, R. et al. Prescription Drug Use and Polypharmacy Among Medicaid-Enrolled Adults with Autism: A Retrospective Cross-Sectional Analysis. Drugs - Real World Outcomes 3, 409-425 (2016).

6. Murray, M. L. et al. Pharmacological treatments prescribed to people with autism spectrum disorder (ASD) in primary health care. Psychopharmacology (Berl.) 231, 1011-1021 (2014).

7. Alfageh, B. H. et al. Psychotropic Medication Prescribing for Neuropsychiatric Comorbidities in Individuals Diagnosed with Autism Spectrum Disorder (ASD) in the UK. J. Autism Dev. Disord. 50, 625-633 (2020).

8. Jobski, K., Höfer, J., Hoffmann, F. \& Bachmann, C. Use of psychotropic drugs in patients with autism spectrum disorders: a systematic review. Acta Psychiatr. Scand. 135, 8-28 (2017).

9. Salomone, E. et al. Use of early intervention for young children with autism spectrum disorder across Europe. Autism Int. J. Res. Pract. 20, 233-249 (2016).
10. NICE. Autism spectrum disorder in under 19s: support and management [CG170]. https://www.nice.org.uk/guidance/ cg170; Accessed 24 May 2020.

11. NICE. Autism spectrum disorder in adults: diagnosis and management [CG142]. https://www.nice.org.uk/ guidance/CG142/; Accessed 24 May 2020.

12. NICE. Depression in adults: treatment and management: NICE guideline short version - Draft for second consultation. https://www.nice.org.uk/guidance/ GID-CGWAVE0725/documents/shortversion-of-draft-guideline; Accessed 24 May 2020.

13. NICE. Depression in children and young people: identification and management [NG134]. https://www.nice.org.uk/ guidance/ng134; Accessed 24 May 2020.

14. NICE. Attention deficit hyperactivity disorder: diagnosis and management [NG87]. https://www.nice.org.uk/ guidance/ng87; Accessed 24 May 2020.

15. Volkmar, F. et al. Practice parameter for the assessment and treatment of children and adolescents with autism spectrum disorder. J. Am. Acad. Child Adolesc. Psychiatry 53, 237-257 (2014).

16. Unigwe, S. et al. GPs' confidence in caring for their patients on the autism spectrum: an online self-report study. Br. J. Gen. Pract. 67, e445-e452 (2017).

17. Coleman-Fountain, E., Buckley, C. \& Beresford, B. Improving mental health in autistic young adults: a qualitative study exploring help-seeking barriers in UK primary care. Br. J. Gen. Pract. 70, e356e363 (2020).

18. Griffith, G. M., Totsika, V., Nash, S. \& Hastings, R. P. 'I just don't fit anywhere': support experiences and future support needs of individuals with Asperger syndrome in middle adulthood. Autism 16, 532-546 (2012). 
19. Carbone, P. S. et al. Parent and pediatrician perspectives regarding the primary care of children with autism spectrum disorders. J. Autism Dev. Disord. 43, 964-972 (2013).

20. Chiri, G. \& Warfield, M. E. Unmet need and problems accessing core health care services for children with autism spectrum disorder. Matern. Child Health J. 16, 1081-1091 (2012).

21. Golnik, A., Ireland, M. \& Borowsky, I. W. Medical homes for children with autism: a physician survey. Pediatrics 123, $966-$ 971 (2009).

22. Scalli, L. E. Accessibility to Health Care Services for Children with Autism Spectrum Disorders [Thesis: Waldon University, 2018]. https://scholarworks. waldenu.edu/cgi/viewcontent. cgi?article $=6801 \&$ context $=$ dissertations; Accessed 20 Jul 2020.

23. Pellicano, E., Dinsmore, A. \& Charman, T. A future made together: shaping autism research in the UK. Centre for Research in Autism and Education, University of London, London UK (2013). https://discovery.ucl.ac.uk/id/ eprint/1495583/1/A_Future_Made_ Together_1.2_LR.pdf; Accessed $16 \mathrm{Jul}$ 2020.

24. Office of Autism Research Coordination, National Institute of Mental Health, on behalf of the Interagency, Autism Coordinating Committee (IACC) \& Interagency Autism Coordinating Committee. 2016 IACC Autism Spectrum Disorder Research Portfolio Analysis Report. https://iacc.hhs.gov/ portfolio-analysis/2016/index.shtml; Accessed 31 May 2020.

25. American Psychological Association. Survey: Americans Becoming More Open About Mental Health. https:// www.apa.org/news/press/releases/apamental-health-report.pdf; Accessed 28 May 2020.
26. Dean, L. \& Phillips, M. Attitudes to mental health problems and mental wellbeing Findings from the 2015 British Social Attitudes survey; Public Health England. (2015).

27. Robinson, E. J. \& Henderson, C. Public knowledge, attitudes, social distance and reporting contact with people with mental illness 2009-2017. Psychol. Med. 49, 2717-2726 (2019).

28. Gulliver, A., Griffiths, K. M. \& Christensen, H. Perceived barriers and facilitators to mental health help-seeking in young people: a systematic review. BMC Psychiatry 10, 113 (2010).

29. Baron-Cohen, S. Editorial Perspective: Neurodiversity - a revolutionary concept for autism and psychiatry. J. Child Psychol. Psychiatry 58, 744-747 (2017).

30. Opar, A. In search of truce in the autism wars (24 April 2019). https://www. spectrumnews.org/features/deep-dive/ search-truce-autism-wars/; Accessed 01 Jun 2020.

31. Stenson, J. Why the focus of autism research is shifting away from searching for a 'cure' (22 Sept 2019). https://www. nbcnews.com/health/kids-health/cureautism-not-so-fast-n1055921; Accessed 01 Jun 2020.

32. Schmid, I., Burcu, M. \& Zito, J. M. Medicaid Prior Authorization Policies for Pediatric Use of Antipsychotic Medications. JAMA 313, 966-968 (2015).

33. American Psychiatric Association. Choosing Wisely - Five Things Physicians and Patients Should Question. https:// www.choosingwisely.org/societies/ american-psychiatric-association/; Accessed 01 Jun 2020.

34. NHS England. Supporting Treatment and Appropriate Medication in Paediatrics (STAMP). https://www.england.nhs.uk/ learning-disabilities/improving-health/ stamp/; Accessed 28 May 2020. 
35. Steinhausen, H.-C. Recent international trends in psychotropic medication prescriptions for children and adolescents. Eur. Child Adolesc. Psychiatry 24, 635-640 (2015).

36. Kalverdijk, L. J. et al. A multi-national comparison of antipsychotic drug use in children and adolescents, 2005-2012. Child Adolesc. Psychiatry Ment. Health 11, 55 (2017).

37. Halfdánarson, Ó. et al. International trends in antipsychotic use: A study in 16 countries, 2005-2014. Eur. Neuropsychopharmacol. 27, 1064-1076 (2017).

38. Donohue, J.M., Cevasco, M. \& Rosenthal, M. B. A decade of direct-to-consumer advertising of prescription drugs. N. Engl. J. Med. 357, 673-681 (2007).

39. Hollon, M. F. Direct-to-consumer marketing of prescription drugs: a current perspective for neurologists and psychiatrists. CNS Drugs 18, 69-77 (2004).

40. Reast, J. D., Palihawadana, D. \& SpickettJones, G. UK physicians. attitudes towards direct-to-consumer advertising of prescription drugs: an extension and review. Int. J. Advert. 23, 229-251 (2004).

41. Reast, J., Palihawadana, D. \& Shabbir, $H$. The Ethical Aspects of Direct to Consumer Advertising of Prescription Drugs in the United Kingdom: Physician versus Consumer Views. J. Advert. Res. 48, 450-464 (2008).

42. Cataife, G. \& Weinberg, D. A. Racial and Ethnic Differences in Antipsychotic Medication Use Among Children Enrolled in Medicaid. Psychiatr. Serv. 66, 946-951 (2015).

43. Cummings, J. R., Ji, X., Allen, L., Lally, C.\& Druss, B. G. Racial and Ethnic Differences in ADHD Treatment Quality Among Medicaid-Enrolled Youth. Pediatrics 139, (2017).

44. Cummings, J. R., Ji, X., Lally, C. \& Druss, B. G. Racial and Ethnic Differences in Minimally Adequate Depression Care Among Medicaid-Enrolled Youth. J. Am. Acad. Child Adolesc. Psychiatry 58, 128138 (2019).
45. Fadus, M. C. et al. Unconscious Bias and the Diagnosis of Disruptive Behavior Disorders and ADHD in African American and Hispanic Youth. Acad. Psychiatry 44, 95-102 (2020).

46. Lord, C., Elsabbagh, M., Baird, G. \& Veenstra-Vanderweele, J. Autism spectrum disorder. The Lancet 392, 508-520 (2018).

47. Keenan, M. et al. Autism and ABA: The Gulf Between North America and Europe. Rev. J. Autism Dev. Disord. 2, 167-183 (2015).

48. Wang, L., Mandell, D. S., Lawer, L., Cidav, Z. \& Leslie, D. L. Healthcare service use and costs for autism spectrum disorder: a comparison between medicaid and private insurance. J. Autism Dev. Disord. 43, 1057-1064 (2013).

49. Zhang, W. \& Baranek, G. The Impact of Insurance Coverage Types on Access to and Utilization of Health Services for U.S. Children With Autism. Psychiatr. Serv. 67, 908-911 (2016).

50. Candon, M. K. et al. The Differential Effects of Insurance Mandates on Health Care Spending for Children's Autism Spectrum Disorder. Med. Care 56, 228232 (2018).

51. Maglione, M. A. et al. Nonmedical interventions for children with ASD: recommended guidelines and further research needs. Pediatrics 130 Suppl 2, S169-178 (2012).

52. Liu, Y. et al. Global birth prevalence of congenital heart defects 1970-2017: updated systematic review and metaanalysis of 260 studies. Int. J. Epidemiol. 48, 455-463 (2019).

53. Perrin, J. M., Friedman, R. A., Knilans, T. K., Group, the B. B. W. \& Surgery, the S. on $\mathrm{C}$. and $\mathrm{C}$. Cardiovascular Monitoring and Stimulant Drugs for AttentionDeficit/Hyperactivity Disorder. Pediatrics 122, 451-453 (2008).

54. Vetter, V. L. et al. Cardiovascular Monitoring of Children and Adolescents With Heart Disease Receiving Medications for Attention Deficit/ 
Hyperactivity Disorder: A Scientific Statement From the American Heart Association Council on Cardiovascular Disease in the Young Congenital Cardiac Defects Committee and the Council on Cardiovascular Nursing. Circulation 117, 2407-2423 (2008).

55. Zito, J. M. \& Burcu, M. Stimulants and Pediatric Cardiovascular Risk. J. Child Adolesc. Psychopharmacol. 27, 538-545 (2016).

56. fda.gov. FDA Drug Safety Communication: Safety Review Update of Medications used to treat AttentionDeficit/Hyperactivity Disorder (ADHD) in children and young adults. https:// www.fda.gov/drugs/drug-safetyand-availability/fda-drug-safetycommunication-safety-review-updatemedications-used-treat-attention; Accessed 30 May 2020.

57. Strattera. STRATTERA® (atomoxetine hydrochloride) CAPSULES for Oral Use Highlights of Prescribing Information (Reference ID: 2914553). https://www. accessdata.fda.gov/drugsatfda_docs/ label/2011/021411s035lbl.pdf; Accessed 30 May 2020.

58. Adderall. Adderall ${ }^{\circledR}$ CII (Dextroamphetamine Saccharate, Amphetamine Aspartate, Dextroamphetamine Sulfate and Amphetamine Sulfate Tablets). https://www.accessdata. fda.gov/drugsatfda_docs/label/ 2017/011522s043lbl.pdf; Accessed 30 May 2020 (2017).

59. Ritalin. Ritalin ${ }^{\circledR}$ hydrochloride methylphenidate hydrochloride (Reference ID: 3421576). https:// www.accessdata.fda.gov/drugsatfda docs/label/2013/010187s077lbl.pdf; Accessed 30 May 2020.

60. Focalin. Focalin ${ }^{\circledR}$ dexmethylphenidate hydrochloride tablets - prescribing Information (Reference ID: 2872329). https://www.accessdata.fda.gov/drugs atfda_docs/label/2010/021278s013lbl. pdf; Accessed 30 May 2020.
61. Winterstein, A. G. et al. Cardiovascular safety of central nervous system stimulants in children and adolescents: population based cohort study. BMJ 345, e4627 (2012).

62. Cooper, W. O. et al. ADHD Drugs and Serious Cardiovascular Events in Children and Young Adults. N. Engl. J. Med. 365, 1896-1904 (2011).

63. di Filippo, L., Doga, M., Resmini, E. \& Giustina, A. Hyperprolactinemia and bone. Pituitary 23, 314-321 (2020).

64. Lally, J., Sahl, A. B., Murphy, K. C., Gaughran, F. \& Stubbs, B. Serum Prolactin and Bone Mineral Density in Schizophrenia: A Systematic Review. Clin. Psychopharmacol. Neurosci. Off. Sci. J. Korean Coll. Neuropsychopharmacol. 17, 333-342 (2019).

65. Lamberti, M. et al. Head-to-Head Comparison of Aripiprazole and Risperidone in the Treatment of ADHD Symptoms in Children with Autistic Spectrum Disorder and ADHD: A Pilot, Open-Label, Randomized Controlled Study. Paediatr. Drugs 18, 319-329 (2016).

66. DeVane, C. L. et al. Pharmacotherapy of Autism Spectrum Disorder: Results from the Randomized BAART Clinical Trial. Pharmacother. J. Hum. Pharmacol. Drug Ther. 39, 626-635 (2019).

67. Shea, S. et al. Risperidone in the treatment of disruptive behavioral symptoms in children with autistic and other pervasive developmental disorders. Pediatrics 114, e634-641 (2004).

68. McCracken, J. T. et al. Risperidone in children with autism and serious behavioral problems. N. Engl. J. Med. 347, 314-321 (2002).

69. Marcus, R. N.et al. A Placebo-Controlled, Fixed-Dose Study of Aripiprazole in Children and Adolescents With Irritability Associated With Autistic Disorder. J. Am. Acad. Child Adolesc. Psychiatry 48, 1110-1119 (2009). 
70. Owen, R. et al. Aripiprazole in the treatment of irritability in children and adolescents with autistic disorder. Pediatrics 124, 1533-1540 (2009).

71. SPARK Consortium. SPARK: A US Cohort of 50,000 Families to Accelerate Autism Research. Neuron 97, 488-493 (2018).

72. Mazurek, M. O. et al. Construct Validity of the Autism Impact Measure (AIM). J. Autism Dev. Disord. 50, 2307-2319 (2020).

73. Mazefsky, C. A. et al. Development of the Emotion Dysregulation Inventory: A PROMIS®ing Method for Creating Sensitive and Unbiased Questionnaires for Autism Spectrum Disorder. J. Autism Dev. Disord. 48, 3736-3746 (2018).

74. Kleinbaum, D., Kupper, L. \& Morgenstern, H. Epidemiologic research. (ISBN: 978-0471-28985-2; Belmont, CA; Lifetime Learning Publications, 1982).

75. Schwartz, S., Campbell, U. B., Gatto, N. M. \& Gordon, K. Toward a Clarification of the Taxonomy of "Bias" in Epidemiology Textbooks. Epidemiology 26, 216-222 (2015).

76. American Psychiatric Association. Diagnostic and Statistical Manual of Mental Disorders (5th ed.). (Washington, DC; Author, 2013).

77. American Psychiatric Association. Diagnostic and Statistical Manual of Mental Disorders (4th ed. text revised). (Washington, DC; Author, 2000).

78. Asperger, H. Die autistichen psychopathen im kindesalter. Arch. Für Psychiatr. Nervenkrankenheiten 76-136 (1944).

79. Asperger, H. \& Frith (Translation), U. 'Autistic psychopathy' in childhood. in Autism and Asperger Syndrome 37-92 (Cambridge University Press, 1991).

80. Fombone, E. Prevalence of childhood disintegrative disorder. Autism Int. J. Res. Pract. 6, 149-157 (2002).

81. Anderson, C. Redefinition: Autism, Asperger's, and the DSM-5 | Interactive Autism Network. https://iancommunity. org/cs/simons_simplex_community/ dsm5_and_asd; Accessed 28 Jun 2020.
82. American Psychiatric Association. DSM-5 Autism Spectrum Disorder Fact Sheet. www.psychiatry.org/File\%20 Library/Psychiatrists/Practice/DSM/ APA_DSM-5-Autism-SpectrumDisorder.pdf; Accessed 28 Jun 2020.

83. Augustyn, M. \& Erik von Hahn, L. Autism spectrum disorder: Evaluation and diagnosis. UpToDate https:// www.uptodate.com/contents/autismspectrum-disorder-evaluation-anddiagnosis; Accessed 08 July 2020.

84. Lord, C.etal. Autism diagnostic observation schedule, second edition. (Western Psychological Services, 2012).

85. Rutter, M., LeCouteur, A. \& Lord, C. (ADIR) Autism Diagnostic Interview-Revised. (Western Psychological Services, 2003).

86. Dawkins, T., Meyer, A. T. \& Van Bourgondien, M. E. The Relationship Between the Childhood Autism Rating Scale: Second Edition and Clinical Diagnosis Utilizing the DSM-IV-TR and the DSM-5. J. Autism Dev. Disord. 46, 3361-3368 (2016).

87. Burke, J. P. et al. Does a claims diagnosis of autism mean a true case? Autism Int. J. Res. Pract. 18, 321-330 (2014).

88. Fombonne, E. et al. Validation of the diagnosis of autism in general practitioner records. BMC Public Health 4, 5 (2004).

89. Hagberg, K. W. \& Jick, S. S. Validation of autism spectrum disorder diagnoses recorded in the Clinical Practice Research Datalink, 1990-2014. Clin. Epidemiol. 9, 475-482 (2017).

90. Smeeth, L. et al. Rate of first recorded diagnosis of autism and other pervasive developmental disorders in United Kingdom general practice, 1988 to 2001. BMC Med. 2, 39 (2004).

91. Health \& Social Care Information Centre. SNOMED to replace Read Codes by 2020. https://www.digitalhealth. net/2015/10/snomed-to-replace-readcodes-by-2020/ (2015).

92. Hayes, J., Ford, T., Rafeeque, H. \& Russell, G. Clinical practice guidelines for diagnosis of autism spectrum disorder in adults and children in the UK: a narrative review. BMC Psychiatry 18, 222 (2018). 
93. Zeldovich, L. Autism diagnosis - The evolution of 'autism' as a diagnosis, explained. Spectrum News https://www. spectrumnews.org/news/evolutionautism-diagnosis-explained/; Accessed 29 Jun 2020.

94. Glover, G. et al. Prescribing of psychotropic drugs to people with learning disabilities and/or autism by general practitioners in England. Public Health England, UK http://clok.uclan. ac.uk/17970/1/Psychotropic\%20 medication\%20and\%20people\%20 with\%20learning\%20disabilities\%20 or\%20autism.pdf; Accessed 06 Jul 2020.

95. Public Health England. Psychotropic drugs and people with learning disabilities or autism: introduction. https://www. gov.uk/government/publications/ psychotropic-drugs-and-peoplewith-learning-disabilities-or-autism/ psychotropic-drugs-and-people-withlearning-disabilities-or-autismintroduction\#aims-and-objectives; Accessed 07 Jun 2020.

96. Truven Health Analytics. Truven Health MarketScan Database: User guide. (2016).

97. Choma, N. N. et al. An algorithm to identify incident myocardial infarction using Medicaid data. Pharmacoepidemiol. Drug Saf. 18, 1064-1071 (2009).

98. Hennessy, S. et al. Validation of diagnostic codes for outpatient-originating sudden cardiac death and ventricular arrhythmia in Medicaid and Medicare claims data. Pharmacoepidemiol. Drug Saf. 19, 555562 (2010).

99. McCormick, N., Lacaille, D., Bhole, V. \& Avina-Zubieta, J.A. Validity of Myocardial Infarction Diagnoses in Administrative Databases: A Systematic Review. PLOS ONE 9, e92286 (2014).

100. McCormick, N., Bhole, V., Lacaille, D. \& Avina-Zubieta, J. A. Validity of Diagnostic Codes for Acute Stroke in Administrative Databases: A Systematic Review. PLOS ONE 10, e0135834 (2015).
101. Kumamaru, H. et al. Validity of claimsbased stroke algorithms in contemporary Medicare data: reasons for geographic and racial differences in stroke (REGARDS) study linked with medicare claims. Circ. Cardiovasc. Qual. Outcomes 7, 611-619 (2014).

102. Wright, N. C. et al. The Design and Validation of a New Algorithm to Identify Incident Fractures in Administrative Claims Data. J. Bone Miner. Res. 34, 17981807 (2019).

103. Ray, W. A., Griffin, M. R., Fought, R. L. \& Adams, M. L. Identification of fractures from computerized Medicare files. J. Clin. Epidemiol. 45, 703-714 (1992).

104. Jean, S. et al. Algorithms can be used to identify fragility fracture cases in physician-claims databases. Osteoporos. Int. 23, 483-501 (2012).

105. Hudson, M. et al. The validity of administrative data to identify hip fractures is high-a systematic review. J. Clin. Epidemiol. 66, 278-285 (2013).

106. Hedström, E. M., Svensson, O., Bergström, U. \& Michno, P. Epidemiology of fractures in children and adolescents. Acta Orthop. 81, 148-153 (2010).

107. Steiner, A. M., Koegel, L. K., Koegel, R. L. \& Ence, W. A. Issues and Theoretical Constructs Regarding Parent Education for Autism Spectrum Disorders. J. Autism Dev. Disord. 42, 1218-1227 (2012).

108. Ryan, C. L. \& Bauman, K. Educational Attainment in the United States: 2015 (US Census Bureau). https://www. census.gov/content/dam/Census/ library/publications/2016/demo/p20578.pdf; Accessed 13 Apr 2020.

109. Guzman, G. Household Income: 2018 -American Community Survey Briefs (US Census Bureau). https://www.census. gov/content/dam/Census/library/ publications/2019/acs/acsbr18-01.pdf; Accessed 07 Apr 2020.

110. Montes, G. \& Halterman, J. S. Association of childhood autism spectrum disorders and loss of family income. Pediatrics 121, e821-826 (2008). 
111. Rogge, N. \& Janssen, J. The Economic Costs of Autism Spectrum Disorder: A Literature Review. J. Autism Dev. Disord. 49, 2873-2900 (2019).

112. Liptak, G. S. et al. Disparities in diagnosis and access to health services for children with autism: data from the National Survey of Children's Health. J. Dev. Behav. Pediatr. JDBP 29, 152-160 (2008).

113. United States Census Bureau. Detailed Languages Spoken at Home and Ability to Speak English for the Population 5 Years and Over: 2009-2013. (2015).

114. Data Resource Center for Child \& Adolescent Health. National Survey of Children's Health (2016 - present). https://www.childhealthdata.org/ browse/survey; Accessed 27 Jun 2020.

115. Pew Research Center. Internet/ Broadband Fact Sheet. https://www. pewresearch.org/internet/fact-sheet/ internet-broadband/; Accessed 27 Jun 2020.

116. Faich, G. Kidney Failure and Analgesic Drugs. N. Engl. J. Med. 1514-1516 (1995).

117. Joseph, K. S., Mehrabadi, A. \& Lisonkova, S. Confounding by Indication and Related Concepts. Curr. Epidemiol. Rep. 1, 1-8 (2014).

118. Langholz, B. \& Goldstein, L. Risk Set Sampling in Epidemiologic Cohort Studies. Stat. Sci. 11, 35-53 (1996).

119. Schneeweiss, S. \& Suissa, S. Discussion of Schuemie et al: 'A plea to stop using the case-control design in retrospective database studies'. Stat. Med. 38, 42094212 (2019).

120. Schuemie, M. J. et al. A plea to stop using the case-control design in retrospective database studies. Stat. Med. 38, 41994208 (2019).

121. Habel, L. A. et al. ADHD Medications and Risk of Serious Cardiovascular Events in Young and Middle-aged Adults. JAMA 306, 2673 (2011).

122. Whitaker, H. J., Farrington, C. P., Spiessens, B. \& Musonda, P. Tutorial in biostatistics: the self-controlled case series method. Stat. Med. 25, 1768-1797 (2006).
123. Petersen, I., Douglas, I. \& Whitaker, H. Self controlled case series methods: an alternative to standard epidemiological study designs. BMJ 354, i4515 (2016).

124. Suissa, S. Immeasurable time bias in observational studies of drug effects on mortality. Am. J. Epidemiol. 168, 329-335 (2008).

125. Lipsitch, M., Tchetgen Tchetgen, E. \& Cohen, T. Negative Controls: A Tool for Detecting Confounding and Bias in Observational Studies. Epidemiology 21, 383-388 (2010).

126. Hernán, M. A. \& Robins, J. M. Using Big Data to Emulate a Target Trial When a Randomized Trial Is Not Available. Am. J. Epidemiol. 183, 758-764 (2016).

127. Ray, W. A. Evaluating medication effects outside of clinical trials: new-user designs. Am. J. Epidemiol. 158, 915-920 (2003).

128. Hernán, M. A. et al. Observational studies analyzed like randomized experiments: an application to postmenopausal hormone therapy and coronary heart disease. Epidemiol. Camb. Mass 19, $766-$ 779 (2008).

129. Potthoff, R. F. Differential losses to follow-up that are outcome-dependent can vitiate a clinical trial: Simulation results. J. Biopharm. Stat. 28, 633-644 (2018).

130. Fine, J. P. \& Gray, R. J. A Proportional Hazards Model for the Subdistribution of a Competing Risk. J. Am. Stat. Assoc. 94, 496-509 (1999).

131. Noordzij, M. et al. When do we need competing risks methods for survival analysis in nephrology? Nephrol. Dial. Transplant. Off. Publ. Eur. Dial. Transpl. Assoc. - Eur. Ren. Assoc. 28, 2670-2677 (2013).

132. Graham, S. M. et al. Loss to Follow-Up as a Competing Risk in an Observational Study of HIV-1 Incidence. PLOS ONE 8, e59480 (2013).

133. Suissa, S. Immortal time bias in pharmacoepidemiology. Am. J. Epidemiol. 167, 492499 (2008). 
134. Donahue, J. G. et al. Inhaled steroids and the risk of hospitalization for asthma. JAMA 277, 887-891 (1997).

135. Soriano, J. B. et al. Survival in COPD patients after regular use of fluticasone propionate and salmeterol in general practice. Eur. Respir. J. 20, 819-825 (2002).

136. Shintani, A. K., Girard, T. D., Arbogast, P. G., Moons, K. G. M. \& Ely, E. W. Immortal time bias in critical care research: application of time-varying Cox regression for observational cohort studies. Crit. Care Med. 37, 2939-2945 (2009).

137. European Medicines Agency. The European Network of Centres for Pharmacoepidemiology and Pharmacovigilance (ENCePP). Guide on Methodological Standards in Pharmacoepidemiology (Revision 5). EMA/95098/2010 [Section 4.2.3.7]. http://www.encepp.eu/ standards_and_guidances/documents/ ENCePPGuideofMethStandardsinPE_ Rev5.pdf; Accessed 01 Jul 2020.

138. Hernán, M. A., Brumback, B. \& Robins, J. M. Marginal structural models to estimate the causal effect of zidovudine on the survival of HIV-positive men. Epidemiol. Camb. Mass 11, 561-570 (2000).

139. Keil, A. P., Edwards, J. K., Richardson, D. R., Naimi, A. I. \& Cole, S. R. The parametric G-formula for time-to-event data: towards intuition with a worked example. Epidemiol. Camb. Mass 25, 889-897 (2014).

140. VanderWeele, T. J. \& Shpitser, I. On the definition of a confounder. Ann. Stat. 41, 196-220 (2013).

141. Alexander, L. K., Lopes, B., RicchettiMasterson, K. \& Yeatts, K. Confounding Bias, Part I, Second Edition. https://sph. unc.edu/files/2015/07/nciph_ERIC11. pdf; Accessed 14 Apr 2020.

142. Greenland, S., Pearl, J. \& Robins, J. M. Causal diagrams for epidemiologic research. Epidemiol. Camb. Mass 10, 37-48 (1999).
143. VanderWeele, T. J. Principles of confounder selection. Eur. J. Epidemiol. 34, 211-219 (2019).

144. Jager, K. J., Zoccali, C., MacLeod, A. \& Dekker, F. W. Confounding: What it is and how to deal with it. Kidney Int. 73, 256-260 (2008).

145. Textor, J., Hardt, J. \& Knüppel, S. DAGitty: a graphical tool for analyzing causal diagrams. Epidemiol. Camb. Mass 22, 745 (2011).

146. Pearl, J. Causal Diagrams for Empirical Research. Biometrika 82, 669-588 (1995).

147. Shrier, I. \& Platt, R. W. Reducing bias through directed acyclic graphs. BMC Med. Res. Methodol. 8, 70 (2008).

148. Joffe, M. M. \& Rosenbaum, P. R. Invited commentary: propensity scores. Am. J. Epidemiol. 150, 327-333 (1999).

149. Collins, R., Bowman, L., Landray, M. \& Peto, R. The Magic of Randomization versus the Myth of Real-World Evidence. N. Engl. J. Med. 382, 674-678 (2020).

150. Rosenbaum, P. R. \& Rubin, D. B. The central role of the propensity score in observational studies for causal effects. Biometrika 70, 41-55 (1983).

151. Linden, A. \& Yarnold, P. R. Using classification tree analysis to generate propensity score weights. J. Eval. Clin. Pract. 23, 703-712 (2017).

152. Westreich, D., Lessler, J. \& Funk, M. J. Propensity score estimation: machine learning and classification methods as alternatives to logistic regression. J. Clin. Epidemiol. 63, 826-833 (2010).

153. Woodward, M. Epidemiology: Study Design and Data Analysis, Third Edition (Chapter 10.17.10 Interpretation of effects). (ISBN: 978-1-4398-3970-6; CRC Press, 2013).

154. McCaffrey, D. F., Ridgeway, G. \& Morral, A. R. Propensity score estimation with boosted regression for evaluating causal effects in observational studies. Psychol. Methods 9, 403-425 (2004).

155. VanderWeele, T. J. \& Ding, P. Sensitivity Analysis in Observational Research: Introducing the E-Value. Ann. Intern. Med. 167, 268-274 (2017). 
156. Mathur, M. B., Ding, P., Riddell, C. A. \& VanderWeele, T. J. Website and R Package for Computing E-Values. Epidemiol. Camb. Mass 29, e45-e47 (2018).

157. E-value calculator. https://www.evaluecalculator.com/; Accessed 30 Jun 2020.

158. Angrist, J. \& Krueger, A. Instrumental Variables and the Search for Identification: From Supply and Demand to Natural Experiments. J. Econ. Perspect. 15, 69-85 (2001).

159. Labrecque, J. \& Swanson, S. A. Understanding the Assumptions Underlying Instrumental Variable Analyses: a Brief Review of Falsification Strategies and Related Tools. Curr. Epidemiol. Rep. 5, 214-220 (2018).

160. Mackie, T.I., Cook, S., Crystal, S., Olfson, M. \& Akincigil, A. Antipsychotic Use Among Youth in Foster Care Enrolled in a Specialized Managed Care Organization Intervention. J. Am. Acad. Child Adolesc. Psychiatry 59, 166-176.e3 (2020).

161. Maenner, M. J. et al. Prevalence of Autism Spectrum Disorder Among Children Aged 8 Years - Autism and Developmental Disabilities Monitoring Network, 11 Sites, United States, 2016. MMWR Surveill. Summ. 69, 1-12 (2020).

162. Doshi, P., Tilford, J. M., Ounpraseuth, S., Kuo, D. Z. \& Payakachat, N. Do Insurance Mandates Affect Racial Disparities in Outcomes for Children with Autism? Matern. Child Health J. 21, 351-366 (2017).

163. LaClair, M. et al. The effect of Medicaid waivers on ameliorating racial/ethnic disparities among children with autism. Health Serv. Res. 54, 912-919 (2019).

164. Wang, L. et al. Changes in Healthcare Expenditures After the Autism Insurance Mandate. Res. Autism Spectr. Disord. 57, 97-104 (2019).

165. Honsberger, K. \& Van Landeghem, K. State Medicaid Managed Care Enrollment and Design for Children and Youth with Special Health Care Needs - A 50-state Review of Medicaid Managed Care Contracts (2017). https://nashp. org/wp-content/uploads/2017/09/50State-Scan-Issue-Brief.pdf; Accessed 05 Jun 2020.
166. Kelleher, K. J. \& Gardner, W. Out of Sight, Out of Mind - Behavioral and Developmental Care for Rural Children. N. Engl. J. Med. 376, 1301-1303 (2017).

167. Rooksby, M., Elouafkaoui, P., Humphris, G., Clarkson, J. \& Freeman, R. Internetassisted delivery of cognitive behavioural therapy (CBT) for childhood anxiety: systematic review and meta-analysis. J. Anxiety Disord. 29, 83-92 (2015).

168. de Bruin, E. J., Bögels, S. M., Oort, F. J. \& Meijer, A. M. Efficacy of Cognitive Behavioral Therapy for Insomnia in Adolescents: A Randomized Controlled Trial with Internet Therapy, Group Therapy and A Waiting List Condition. Sleep 38, 1913-1926 (2015).

169. Bearss, K. et al. Feasibility of Parent Training via Telehealth for Children with Autism Spectrum Disorder and Disruptive Behavior: A Demonstration Pilot. J. Autism Dev. Disord. 48, 10201030 (2018).

170. Parsons, D., Cordier, R., Vaz, S. \& Lee, H. C. Parent-Mediated Intervention Training Delivered Remotely for Children With Autism Spectrum Disorder Living Outside of Urban Areas: Systematic Review. J. Med. Internet Res. 19, e198 (2017).

171. Iadarola, S. et al. Teaching Parents Behavioral Strategies for Autism Spectrum Disorder (ASD): Effects on Stress, Strain, and Competence. J. Autism Dev. Disord. 48, 1031-1040 (2018).

172. Forehand, R. \& Kotchick, B. A. Cultural Diversity: A Wake-Up Call for Parent Training - Republished Article. Behav. Ther. 47, 981-992 (2016).

173. CPRD. CPRD linked data. https://www. cprd.com/linked-data; Accessed 06 Jun 2020.

174. Newcastle University. Adult Autism Spectrum Cohort - UK. https://research. ncl.ac.uk/adultautismspectrum/; Accessed $01 \mathrm{Jul} 2020$. 
175. Westminster Commission on Autism. A Spectrum of Obstacles - An Inquiry into Access to Healthcare for Autistic People, July 2016, National Childrens Group. https://westminsterautismcommission. files.wordpress.com/2016/03/ar1011_ ncg-autism-report-july-2016.pdf; Accessed 05 Jun 2020.

176. NHS. Annual health checks: learning disabilities. https://www.nhs.uk/ conditions/learning-disabilities/annualhealth-checks; Accessed 06 Jun 2020.

177. NHS. The NHS Long Term Plan. https:// www.longtermplan.nhs.uk/wp-content/ uploads/2019/08/nhs-long-term-planversion-1.2.pdf; Accessed 06 Jun 2020.

178. Buckley, C. Autistic Spectrum Disorders: a clinical priority. https://www.rcgp. org.uk/-/media/Files/CIRC/Autism/ RCGP-Position-Statement-on-AutisticSpectrum-Disorders.ashx? la=en; Accessed 05 Jun 2020.

179. Buckley, C. RCGP Position Statement on Autistic Spectrum Disorders: June 2016. https://www.rcgp.org.uk/ASD; Accessed 05 Jun 2020.

180. Lee, S.-H. et al. Use of antipsychotics increases the risk of fracture: a systematic review and meta-analysis. Osteoporos. Int. 28, 1167-1178 (2017).

181. Papola, D., Ostuzzi, G., Thabane, L., Guyatt, G. \& Barbui, C. Antipsychotic drug exposure and risk of fracture: a systematic review and meta-analysis of observational studies. Int. Clin. Psychopharmacol. 33, 181-196 (2018).

182. Neumeyer, A. M. et al. Brief Report: Bone Fractures in Children and Adults with Autism Spectrum Disorders. J. Autism Dev. Disord. 45, 881-887 (2015).

183. Neumeyer, A. M., Gates, A., Ferrone, C., Lee, H. \& Misra, M. Bone density in peripubertal boys with autism spectrum disorders. J. Autism Dev. Disord. 43, 1623-1629 (2013).

184. Hong, M. P. \& Erickson, C. A. Investigational drugs in early-stage clinical trials for autism spectrum disorder. Expert Opin. Investig. Drugs 28, 709-718 (2019).
185. Liu, C. et al. Longitudinal Change in Core Symptoms of Autism Spectrum Disorder in Children: Online Survey of Caregivers. International Society for Autism Research (INSAR) https://insar. confex.com/insar/2020/intell/eposter. cgi?eposterid=661; Accessed $20 \mathrm{Jul}$ 2020 (2020).

186. Bianchi, S. SPARK pilot study identifies novel candidate autism risk genes. https://www.sfari.org/2019/09/03/ spark-pilot-study-identifies-novelcandidate-autism-risk-genes/; Accessed 24 May 2020.

187. Fombonne, E. et al. Psychiatric and Medical Profiles of Autistic Adults in the SPARK Cohort. J. Autism Dev. Disord. (2020) doi:10.1007/s10803-02004414-6. 



\section{Appendices}

Summary

Valorisation addendum

Databases used

Formal acknowledgements

Personal acknowledgements

List of publications

About the author 


\section{Summary}

This thesis explored utilisation and outcomes of treatment in autism spectrum disorder (ASD). ASD is a lifelong neurodevelopmental disorder, characterised by core symptoms of communication deficits, difficulties with social interaction, and restricted and repetitive behaviours. Associated symptoms of autism include obsessions, self-injury, irritability, aggression, and under-reactivity to sensory stimuli. Psychiatric and neurological conditions such as attention deficit/hyperactivity disorder (ADHD), anxiety, depression, epilepsy and bipolar disorder are also more commonly diagnosed in children and adults with autism than in the general population. ASD is typically diagnosed at age 3-4 years in developed countries. Approximately, three to four times as many boys are diagnosed than girls. Up to 1 in 54 children and 1 in 45 adults are diagnosed with ASD in the USA today.

\section{Chapter 1: Introduction, objectives and data}

Chapter 1 set out the background to this thesis, by characterising the current ASD treatment landscape and some important knowledge gaps.

The purposes of treatment in ASD should be to minimise core and associated ASD impairments, to maximise daily living skills for independence, and to relieve the impact of problem behaviours.

There are currently no pharmacological treatment options for the core symptoms of ASD. In the USA however, the atypical antipsychotics risperidone and aripiprazole are approved for associated symptoms of irritability and aggression in children and adolescents. These drugs been recommended in clinical guidelines for treatment of aggression or selfinjurious behaviours, in order to facilitate daily living, or to allow better adherence to non-drug therapies, but typically only when behavioural interventions have not been successful. These drugs do not have a marketing authorisation in ASD in Europe, yet the United Kingdom National Institute for Health and Care Excellence (NICE) also recommends antipsychotic medications for children with behavioural challenges when non-drug treatments have not helped: so long as these drugs are started by a paediatrician or psychiatrist.

Generally, it is acknowledged that other classes of psychotropic drugs (e.g. antidepressants, anticonvulsants) may be required to manage common psychiatric comorbidities in ASD. A recent systematic review reported median psychotropic drug use estimates of $42 \%$ in children and $62 \%$ in adults in ASD. The systematic review also pointed to some clear gaps in the literature however. Firstly, the vast majority of studies only focused on North America and used data from over a decade ago. There are limited studies in the adult population and studies generally do not have non-ASD comparator groups to contextualise results. 
Drugs used for the treatment of ADHD, such as stimulants and atomoxetine are used frequently in ASD (around 14-19\% in children). These drugs have consistently shown medication-induced increases in blood pressure and heart rate, which coupled with case reports, has raised concerns over the potential increased risk of more serious cardiovascular (SCV) events such as stroke, myocardial infarction, and cardiac arrhythmias. Limited observational studies on this topic have produced inconsistent results. Furthermore, this has not been studied in children with ASD.

Children with ASD also commonly use antipsychotic medication (around 17\%). Antipsychotics have been associated with increased bone fracture risk in elderly dementia patients, yet the mechanisms leading to increased risks is not clear. For example, it may be due to an increased risk of accidents or falling, or due to a negative impact on bone mineral density. The relationship between antipsychotics and fracture risk has not been well studied in children. However, as risperidone and aripiprazole are commonly used in ASD and have slightly different pharmacological profiles, this setting can offer a unique opportunity to understand possible mechanisms and inform relevant clinical decisions about which treatment to prescribe.

The mainstay of currently recommended treatments for ASD are non-pharmacological in nature, and include behavioural and social-communication based therapies. In a study across 18 European countries, 91\% of children with ASD received at least one type of non-drug intervention by age 7 years. Historical uptake of non-drug treatments is also high in the USA, with up to $77 \%$ current use. However, there are concerns that children in more rural settings have access to fewer services. Additionally, healthcare expenditures have previously been higher for ASD children with public versus private health insurance, suggesting public insurance may be an advantage for accessing services.

While a number of diagnostic instruments are available to aid a professional diagnosis of ASD, there is little consensus on the most appropriate tools for measuring effects of treatment. Patient or caregiver-reported assessments can offer a quick and cheap option to collect patient symptoms. They may also be administered remotely, for example on a computer or mobile phone. Hence, provided they are validated, they can offer a more sustainable opportunity to evaluate treatment effectiveness over longer periods, and outside controlled clinical trial settings.

This thesis comprised a collection of studies to address various abovementioned knowledge gaps regarding the utilisation and outcomes of treatment in ASD. The objectives were grouped into the following three categories: treatment utilisation patterns, treatment safety, and validation of a new caregiver-reported measure of ASD symptom severity. These categories correspond to Chapters 2 to 4 of the thesis respectively. 
We used three main sources of real world data throughout the thesis. The first was the MarketScan insurance database which covers de-identified patient-level health data from private ("commercial") and publically (Medicaid) insured populations in the USA. The data covers all billed episodes of care, regardless of the setting. The second database was the Clinical Practice Research Datalink (CPRD) electronic medical record database from the UK. Data is recorded in the primary care setting, although due to the nature of the general practitioner (GP) "gatekeeper" system in the UK, prescriptions should be managed (or at least documented) by the GP even if they started in secondary or tertiary care. The third database was collected via a primary data collection survey study, nested in the Simons Foundation Powering Autism Research for Knowledge (SPARK) platform. SPARK is an online, USA-based research initiative for individuals with ASD and their family members.

\section{Chapter 2: Treatment utilisation in ASD}

Chapter 2 focused on the production of up to date estimates of drug and non-drug treatment utilisation in ASD, as well as predictors of use.

Chapter 2.1 was a retrospective, cross-sectional cohort study based in USA MarketScan claims in calendar year 2014. Among 46,943 commercial- and 46,696 Medicaid-insured patients we found substantial annual proportions of psychotropic drug use (64\% and 69\% respectively). These proportions increased rapidly throughout childhood from 11-25\% in the 3-4 years age group to $76-80 \%$ in the 12-17 years age group. In adulthood, the proportions roughly plateaued (78-81\%). All age groups combined, the most commonly used treatments (commercial-Medicaid) were stimulants (30-32\%), antipsychotics (25-35\%), antidepressants (33-29\%) and hypotensive agents (20-31\%). The rate of polypharmacy (two or more treatments concurrently for 30 days) was also high and increased with age (35-44\% for all age groups combined, but already 24-36\% by age 5-11 years). Medications were most frequently prescribed in conjunction with the indicated psychiatric condition, although over $30 \%$ of participants received medication in the absence of a coded psychiatric condition other than ASD. Beyond age and comorbidities, we also found that fee-for-service insurance plans, foster care and White race were associated with higher treatment rates.

Chapter 2.2 was a retrospective, cross-sectional cohort study based in the UK CPRD database in calendar year 2015. Among 10,856 patients, 32\% used a psychotropic drug. This is around half the annual proportion observed in the USA, but substantial nonetheless. Also unlike in the USA, treatment rates increased gradually throughout childhood and adulthood years, with small increases in prevalence rate at each increasing age group (11\% at age $3-4$ years, $26 \%$ at age $12-17$ years and $44 \%$ in adulthood). All age groups combined, the most commonly used treatments were anxiolytics/sedatives/ hypnotics (14\%), antidepressants (13\%) and antipsychotics (8\%). Hypotensive agents were used by less than $1 \%$ of patients. The rate of polypharmacy was $10 \%$ overall, and also increased gradually with age. Presence of psychiatric comorbidities was associated 
with treatment use, but similar to the USA, some patents (14\%) received psychotropic medication despite having no record of corresponding psychiatric comorbidities. Beyond age and comorbidities, females were more likely to be treated than males. In age, sex, and region matched comparative analysis, we found that psychotropic treatment use, polypharmacy, and healthcare resource use were all substantially higher in ASD than in the general population. However, odds for these outcomes were roughly halved in ASD versus an ADHD-matched cohort. This was primarily driven by the higher frequency of stimulant prescriptions in ADHD (35\%) versus ASD (7\%).

Chapter 2.3 was a survey study of parents and caregivers of children aged 3-17 years with ASD. The survey was embedded as part of the SPARK platform. We received responses from 5,122 caregivers (45\% of invited) on questions regarding their child's use, setting and barriers to care for different non-drug treatment types over the past year (roughly September 2016 to September 2017). Overall, $96 \%$ of children received at least one type of non-drug treatment, with the most common being speech and language therapy (SLT; 71\%), occupational therapy (OT; 60\%) and behavioural therapy (56\%). Around 30\% of caregivers attended caregiver-training courses. The median intensity of all child-directed treatments combined was 6 hours per week, with behavioural therapy being the most intense (4 hours per week). All treatments were more commonly given in individual rather than group sessions. SLT and OT were more often provided in school, while behavioural therapy and psychological interventions were more frequently provided outside school. Controlling for other factors, behavioural therapy and SLT were significantly more frequent and more intense in metropolitan (urban) than in nonmetropolitan (rural) areas (odds ratios and 95\% confidence intervals (OR) were 1.54 (1.30-1.83) for behavioural therapy, and 1.41 (1.17-1.69) for SLT). There were no consistently significant associations between non-drug treatment use and type of insurance coverage (commercial or Medicaid).

\section{Chapter 3: Safety evaluation of treatments in children}

Chapter 3 explored safety concerns of two commonly used drug classes inchildren with ASD.

Chapter 3.1 consisted of two case-control studies, nested within respective cohorts of ADHD and ASD children aged 3-18 years. In these groups, we evaluated the relationship between serious cardiovascular (SCV) events and current use of ADHD medications. ADHD medications were atomoxetine and stimulants (such as amphetamine, methylphenidate, lisdexamfetamine). We defined cases by a composite SCV outcome of stroke, myocardial infarction, or serious cardiac arrhythmia, and for each case, we matched ten controls on age, sex, and insurance type. We used the MarketScan commercial (years 2000-2016) and Medicaid (years 2012-2016) data. We identified 2,240,774 children for the ADHD cohort and 326,221 children for the ASD cohort. Overall, incidence rates of SCV events was extremely low: 3.1 per 100,000 patient years in the ADHD cohort and 5.6 per 100,000 patient years in the ASD cohort. For ADHD, 33.9\% of cases (63 of 186 ) versus $32.2 \%$ of controls (598 of 1,860 ) were exposed, which yielded an OR of 
1.08 (0.78-1.49). For ASD, 12.5\% of cases (6 of 48) versus $22.1 \%$ of controls (106 of 480) were exposed (OR 0.49 (0.20-1.20)). Covariate-adjusted results and results for individual outcomes and other exposure definitions were also consistent with no increased risk of SCV events. In short, we found no evidence of increased SCV risk in children and adolescents with ADHD or ASD exposed to ADHD medications.

Chapter 3.2 was a propensity score matched cohort study to compare the risk of fracture among children aged 2-18 years with ASD using either risperidone or aripiprazole. We identified 3,312 new users of each drug from the MarketScan Medicaid data between years 2013 and 2018. The main exposure was continued use of aripiprazole or risperidone over time. Over the full duration of follow-up (median 10 months in both cohorts), incidence rates of any fracture per 1,000 patient-years were 23.2 for risperidone and 38.4 for aripiprazole. The hazard ratio and 95\% confidence interval (HR) was 0.60 (0.440.83). Risks were similar between cohorts throughout the first 180 days on treatment, but significantly higher in the aripiprazole group thereafter. Extremity fractures drove most of the increased risk, with the biggest differences in lower leg and ankle fractures. Differences widened for children aged 10 years or younger (HR 0.47 (0.30-0.74)). In short, compared to aripiprazole, risperidone was associated with $40 \%$ lower risk of fracture.

\section{Chapter 4: Validation of caregiver reported ASD severity}

Chapter 4 was dedicated to the psychometric validation of the Autism Impact Measure (AIM): a new caregiver reported assessment for the severity of ASD symptoms in children. The AIM was completed online by 5,001 caregivers from the SPARK cohort during September and October 2017, and the study sample are a subset of those caregivers surveyed in Chapter 2.3. Children were aged 3-17 years. This study demonstrated the AIM's ability to discriminate between "known-groups" of children with different symptom severity, estimated thresholds for clinically important responses and confirmed internal and external validity of the measure. We also confirmed meaningful and distinct domains of the AIM including ASD core symptoms of communication deficits, difficulties with social interactions and repetitive behaviours. Importantly, this study showed the AIM is a valid tool, which can be quickly completed in a remote setting by caregivers on computer and mobile devices (median time: 7 minutes).

\section{Chapter 5: General discussion}

Chapter 5 provided a summary and critical reflection on the main body of the thesis. It included a lengthy summary of the main findings placed into a broader context, a critical evaluation of epidemiological methods used and considerations for future research and implications. Please see the relevant section of this thesis for further details.

\section{Conclusion}

This thesis demonstrates that the treatment landscape in ASD is varied with many drug and non-drug treatment options commonly used. The likelihood of accessing and receiving 
these treatments is dependent on more than just health status, and is also associated with increased age, country of residence, female gender (in the UK), and urbanisation of residence, fee-for-service healthcare plans, race, and foster care status (in the USA). We showed that serious cardiovascular events were not associated with ADHD medication for most children with ASD. Compared to risperidone, long-term aripiprazole use in ASD was associated with increased risk of fractures, especially for children under age 10 years. Finally, the Autism Impact Measure (AIM) offers a valid, quick and inexpensive method for caregivers to report their child's severity of core autism symptoms.

In the coming years, as the treatment landscape in ASD continues to evolve, epidemiology and real world databases (both primary and secondary data use) will continue to compliment clinical trials in helping to understand real life experiences of how people with ASD access and use treatments, as well as the risks and benefits of doing so. 


\section{Valorisation addendum}

Valorisation refers to the process of transferring purely scientific knowledge gained in academic studies into value for broader societal purposes. Results of this thesis are discussed in this context in this appendix.

\section{Healthcare problem}

Autism is a neurodevelopmental condition which can have a tremendous impact on the affected person as well as other members of the family. Around $2 \%$ of people in the USA are diagnosed with autism today and it is known to affect people of all geographies and ethnic, cultural and racial backgrounds. The main symptoms are difficulties with verbal and non-verbal communication, difficulties understanding social or emotional cues, and the desire to carry out simple and repetitive tasks. Other common difficulties - faced by some, but not all people with autism - include attention deficits, depressive thoughts, anxiety, sleep problems, irritable and aggressive behaviours, and obsessions, among others. The wide variety of symptoms in autism is what prompted Dr Stephen Shore - a special education professor with autism - to say, "If you've met one person with autism, you've [only] met one person with autism." In other words, autism effects different people in different ways. This also the reason for the coining of the term autism "spectrum" disorder, or ASD for short.

Today, there are no drugs to treat autism's core symptoms. Instead, the majority of recommended treatments are based on individualised behavioral or social-developmental therapies. There are however concerns that not all children with autism have the same access to such therapies. For example, in the USA, there have been historical concerns that children in rural areas, or on private healthcare plans, find it harder to access these treatments.

The fact that most recommended treatments are non-drug based, does not mean that people with autism do not take drugs as well. In fact, prior to the start of this thesis, estimates were that around $42 \%$ of autistic children and $62 \%$ of autistic adults use psychotropic drugs (drugs that effect the brain). These numbers are mainly based on data from North America however, and the studies on adults did not include many people.

With the widespread use of psychotropic medications in ASD comes concerns about potential safety effects, especially in children. For example, stimulants and atomoxetine (used to manage attention deficits), are known to increase blood pressure and heart rate, which in turn has raised concerns about the possible increased risk of more serious cardiovascular events such as stroke, heart attacks, or severe irregular heartbeats. Antipsychotics (used to manage behavioral difficulties in ASD) have previously been associated with increased bone fracture risk in elderly dementia patients, but it is unclear if this is also the case in children with autism. 
In terms of testing new treatments for autism, there is little agreement on how to measure which treatments are most effective. Measures used in clinical trials are often timeconsuming and expensive. It is often meaningful to understand how effective treatments are in "real-life" settings too, so cheaper and more sustainable ways to measure autism severity are needed. Given that parents of children with autism are often the main organisers and advocates for their child's treatment plan, a questionnaire of autism severity that can be completed by caregivers would be especially meaningful.

\section{Main findings and implications of this thesis}

This thesis comprised a collection of studies to address various problems and unanswered questions related to the use and outcomes of treatments in ASD, as mentioned above. All studies were based on either data from "real-life" settings using data that already existed in electronic medical records or insurance claims data, or by surveying caregivers of children with ASD.

The first set of results in this thesis examined levels of drug and non-drug treatment use in people with ASD. Having reliable treatment utilisation estimates is important for a variety of reasons. They can be used to quantify disease burden, inform healtheconomic and cost-benefit assessments of treatments, inform healthcare resource and training plans, measure the scale of certain risks (e.g. adverse events) and to aid planning for further research (such as defining eligibility criteria and interpreting results of new clinical trials). Other uses include identifying deviations from treatment guidelines or spotting differences in treatment approaches between countries, regions or healthcare plans, which can lead to additional understanding of underlying causes of deviations from guidelines, or if one healthcare system can learn from the other.

In this thesis, we found that drug treatment use is more prevalent in the USA (around $60 \%$ for children and $80 \%$ for adults) than in the UK (24\% for children and $44 \%$ for adults). Differences in regulatory drug approvals likely explain some of the variation, but other factors such as differences in clinical guidelines, attitudes towards receiving drug treatment and differences in healthcare systems all deserve further exploration (which we began to do in Chapter 5). In time, results of our drug utilisation studies may provide important "baseline" data by which to assess the effectiveness of new policies aimed at either increasing or decreasing use of these drugs in the future. For example, since 2016 in the UK, a national program to stop "over medication" of all psychotropic drugs to people with ASD or intellectual disabilities, has been supported by NHS England. Additionally, our findings about other factors associated with increased treatment use, such as foster care and White race in the USA, and female gender in the UK should become the target of other future policy changes, to ensure equitable access to appropriate treatments for all.

For non-drug treatments, caregivers in the USA reported that almost all children (96\%) received a non-drug treatment during a one-year period. We found no significant 
differences in levels of treatment use between private and Medicaid (public) insurances, which is contrary to historical evidence of higher healthcare use in Medicaid, and supports the notion that recently introduced private insurance mandates have been effective at correcting historical advantages for accessing treatments via public plans. This evidence could be used to advocate for the introduction of similar mandates in other disease areas. Regarding geography, a significantly higher proportion of children in metropolitan areas versus non-metropolitan areas received SLT (72\% vs 65\%) and behavioural therapy (57\% vs $46 \%$ ). The most widely reported barrier to care in rural areas was a lack of available local services. Services like telehealth should be considered to address this gap in the future.

The second set of results in this thesis regarded safety concerns, as outlined above. Firstly, in two large case-control studies of children with ASD and attention deficit/hyperactivity disorder, we found no association between serious cardiovascular events and the current use of stimulants or atomoxetine. This finding should reassure the vast majority of physicians and parents, as well as a large number of patients who take these medications (around 14-19\% children use one of these drugs annually). Further observational studies should focus on the subgroup of children with serious underlying cardiac abnormalities who were at increased risk however, and evaluate if their risk is independent of, or compounded by these medications. Until the evidence is clear, physicians should continue to note class-wide warnings and make careful cardiac evaluations in this vulnerable subset of patients prior to prescribing these medications.

Secondly, for fractures, we found a 40\% lower risk of fracture for risperidone-exposed children compared to aripiprazole-exposed children. Risks were comparable between groups for the first 180 days on treatment, but significantly higher in the aripiprazole group thereafter, and even more pronounced for children less than 10 years old. If further studies corroborate our findings then this could deliver unique insights into the mechanisms by which antipsychotics have an impact on bone health, and eventually have impact in prescribing patterns in other disease beyond ASD too (for example, in schizophrenia and dementia). Nonetheless, until more is understood about these mechanisms and patients most at risk, patients and physicians should continue to use aripiprazole as usual but be aware of this signal. The information is certainly important and very clinically relevant, given that around $17 \%$ of autistic children use of these antipsychotics per year in the USA.

A final major contribution of this thesis was the validation of the Autism Impact Measure (AIM) as a reliable tool for measuring the severity of core autism symptoms. Given the variety of available interventions already being used by people with autism, plus new treatments in development, having reliable and accurate ways to compare treatment benefits is of critical importance for future decision making. This includes weighing up the benefits and risks of new treatments, by regulatory authorities, payers and providers of healthcare, and patients themselves. 
As the AIM has distinctive benefits of being caregiver-relevant, quick and inexpensive to administer remotely, then it should be considered as tool for the real world monitoring of ASD symptoms, as well as in clinical trials. Through such a mechanism, the evaluation of different therapeutic approaches can inform treatment guidelines at a group level. It could potentially also allow rapid and direct feedback for effectively finding optimal treatment strategies on the individual level.

For the studies of non-drug treatment utilisation and validation of the AIM, we surveyed parents of children with ASD in the USA, via the Simons Foundation Powering Autism Research for Knowledge (SPARK) platform. SPARK is an online, USA-based research initiative for individuals with ASD and their family members. An intended and valuable benefit of conducting research via the SPARK online platform is that this offers a unique opportunity to engage the autism community directly in research, and to receive feedback on its relevance to them. Of the more than 5,000 caregivers surveyed, our research topic received an average rating of 4.8 out of 5 stars for its importance to them and their family. Once SPARK participant said, "I wanted to thank you for doing this study. We moved to a different state and we found vastly different therapies and available providers. This is a real problem for families."

The fact the SPARK platform as a whole has recruited 60,000 individuals with ASD and their families since forming in 2016 is testament to the incredible engagement and willingness of the ASD community to contribute to further research. All data from our studies in the SPARK cohort will be made available for linkage via the SPARK platform. The opportunity for future studies in the platform, either prospective or retrospective in nature, should not be missed. 


\section{Databases used}

MarketScan is a registered trademark of Truven Health Analytics Inc., an IBM Company.

The Clinical Practice Research Datalink (CPRD) data referred to throughout this thesis is the CPRD GOLD database. For information on other CPRD databases visit https:// www.cprd.com/. CPRD is jointly sponsored by the Medicines and Healthcare products Regulatory Agency and the National Institute for Health Research, as part of the Department of Health and Social Care in the United Kingdom.

The Simons Foundation Powering Autism Research for Knowledge (SPARK) platform is sponsored and operated by Simons Foundation Autism Research Initiative (SFARI). 


\section{Formal acknowledgements}

All studies presented in this thesis were sponsored by F. Hoffmann-La Roche Ltd (Roche). Roche had treatment(s) for autism spectrum disorder in development throughout the conduct of all studies and writing of this thesis.

Related to various studies presented in this thesis, we thank Gonzalo Duran Pacheco (Roche), David Evans (Roche), Kiely Law (Kennedy Krieger Institute and John Hopkins University), Brigitta Monz (Roche), Ajay Patel (Roche) and Frank de Vries (Maastricht UMC+) for providing comments related to clinical and/or statistical expertise. We also thank Yingjie Ding, David Oliveri and Andy Surinach (of Genesis Research under contract with Roche) for quality checking of analysis results. Also related to clinical expertise, we are grateful to Rianne Fokke and Alice Hurlstone for reviewing aspects of the introduction and general discussion of the thesis, respectively. Finally, we thank the team at Tempus Dynamics (under contract with SPARK) for converting the SPARK study questionnaires into an electronic format and managing the study workflow.

We are truly grateful to all the families in SPARK, the SPARK clinical sites and SPARK staff for their participation. 


\section{Personal acknowledgements}

There are a number of people I would like to acknowledge for their part in the process of completing this thesis.

Firstly, thank you to my supervisors, Frank, Joop and Brigitta. Your immense knowledge and guidance throughout has been invaluable. I am forever appreciative for the many selfless hours each of you have spent supporting me. Frank, I am especially grateful for all the motivation and fun you injected into the process, for being a great teacher, and for your dedication, commitment and flexibility, even when having tough times yourself. Brigitta, not only did you take a chance by hiring me as a data-scientist and by wholeheartedly supporting my PhD, but you are a true role model of work ethic and leadership. Joop, thank you so much for the direction and pragmatism, especially for help in getting me over the finishing line.

Secondly, thank you to all my collaborators and colleagues, at Roche, Maastricht and further afield. I have been truly lucky to been surrounded by such smart people and for the opportunities to learn from and be inspired by you all. Thank you too, to the members of the assessment committee, for reviewing my thesis.

Céline, thank you for everything! Throughout this journey you have supported me in so many ways, and listened to hours of me talking about the same old things. You have patience in abundance and would now probably do a good job at defending this thesis yourself!

A sincere thank you too, to all of my friends who have cheered me on, but especially to Chris, whose persistence and stern words pushed me to put pen to paper for the final chapters.

Finally thank you to my family - particularly my parents - for being a constant source of love and support, for instilling in me self-belief, and for encouraging me to pursue my curiosities. To Auntie Angie, Caithan and Cade, you have provided much of the personal inspiration and drive to study this topic. This thesis is for you and other families touched by autism. 


\section{List of publications}

1. Houghton R, de Vries F, and Loss G (2020) Psychostimulants/atomoxetine and serious cardiovascular events in children with ADHD or autism spectrum disorder. CNS Drugs 34, 93-101

2. Monz B, Houghton R, Law K, and Loss G (2019) Treatment patterns in children with autism in the United States. Autism Res. 12, 517-526.

3. Houghton R, Boess F, Verselis L, Ding Y, Freitas R, Constantinovici N, and Ong R (2019) Treatment Patterns in Patients with Incident Parkinson's Disease in the United States. J. Park. Dis. 9, 749-759

4. Houghton R, Monz B, Law K, Loss G, Le Scouiller S, de Vries F, and Willgoss $T$ (2019) Psychometric Validation of the Autism Impact Measure (AIM). J. Autism Dev. Disord. 49, 2559-2570.

5. Houghton R, Liu C, and Bolognani F (2018) Psychiatric Comorbidities and Psychotropic Medication Use in Autism: A Matched Cohort Study with ADHD and General Population Comparator Groups in the United Kingdom. Autism Res. 11, 1690-1700.

6. Houghton R, Ong R, and Bolognani F (2017) Psychiatric comorbidities and use of psychotropic medications in people with autism spectrum disorder in the United States. Autism Res. 10, 2037-2047.

7. Freeman C, Dixon M, Houghton $\mathbf{R}$ et al (2016) Role of CD20 expression and other pre-treatment risk factors in the development of infusion-related reactions in patients with CLL treated with obinutuzumab. Leukemia 30, 1763-1766.

8. Freeman C, Morschhauser F, Sehn L, Dixon M, Houghton $\mathbf{R}$ et al (2015) Cytokine release in patients with CLL treated with obinutuzumab and possible relationship with infusion-related reactions. Blood 126, 2646-2649.
9. Rodrigues F, Byrne L, Tortelli R, Johnson E, Wijeratne P, Arridge M, De Vita E, Ghazaleh N, Houghton R, et al (in press) Longitudinal dynamics of mutant huntingtin and neurofilament light in Huntington's disease: the prospective HD-CSF study. Sci Trans Med.

10. Crowell $\mathrm{V}$, Houghton $\mathbf{R}$, Tomar $\mathrm{A}$, Fernandes T, and Squitieri F (submitted) Modelling the diagnosed prevalence of Huntington's Disease based on diagnosed incidence and survival time.

11. Ghazaleh N, Houghton R, Palermo G, Schobel S, Wijeratne P, Long J (submitted) Ranking the predictive power of clinical and biological features associated with disease progression in Huntington's disease: a data-driven approach using Enroll-HD.

12. Houghton R, van den Bergh J, Law K, Liu $Y$, and de Vries F (submitted). Risperidone versus aripiprazole fracture risk in children and adolescents with autism spectrum disorders. 


\section{About the author}

Richard Houghton was born on 19th May 1991 in Nottingham, United Kingdom. He graduated from Arnold Hill Academy and Sixth Form College in 2009. In July 2013 he obtained a Bachelor's degree in Mathematics (first class honours) from Sheffield Hallam University. During his Bachelor's degree, Richard spent an industry placement year working as a statistical programmer for F. Hoffmann-La Roche Ltd (Roche) in Welwyn Garden City, United Kingdom.

Richard began full time work as a statistical programmer at Roche in July 2013, supporting the analysis of phase 3 clinical trials in rheumatoid arthritis, breast cancer, and chronic lymphocytic leukaemia. In November 2014 he relocated to Basel, Switzerland in order to join the real world data-science team in the Roche headquarter offices, where he is still employed today. He completed a Master's degree in Applied Statistics (distinction) during part-time evening studies at Birkbeck, University of London, United Kingdom, between 2013 and 2016.

Over the past six years at Roche, Richard has supported real world data studies in spinal muscular atrophy, Parkinson's disease, Alzheimer's disease, Huntington's disease and autism spectrum disorder. Beginning in 2017, his work on autism spectrum disorder is what afforded him the opportunity to pursue a PhD by publication at Maastricht University Medical Center, The Netherlands. During this time, Richard was affiliated to the Department of Clinical Pharmacy and Toxicology, Care and Public Health Research Institute (CAPHRI) and the Cardiovascular Research Institute Maastricht (CARIM). His work was academically supervised by Prof. dr. F. de Vries and Prof. dr. J.P.W. van den Bergh, and by Dr. Brigitta Monz from Roche.

Richard has presented work at conferences of the International Society for Pharmacoeconomics and Outcomes Research (ISPOR), the International Conference on Pharmacoepidemiology \& Therapeutic Risk Management (ICPE) and various diseaserelevant medical conferences. His recent work at Roche involved the support of local real world data and epidemiology projects in various developed and developing countries. Since October 2020, Richard has been appointed as Associate Group Director of Data Science at Roche, and currently leads a team of real world data scientists with a focus on neurodegenerative and neuromuscular diseases. 